\title{
AN INVESTIGATIVE STUDY ON NEUTRON EMISSIONS FROM TITANIUM- DEUTERIUM SYSTEM UNDER THERMAL SHOCK
}

\begin{tabular}{c} 
A Dissertation \\
Presented to \\
the faculty of the Graduate School \\
University of Missouri - Columbia \\
In Partial Fulfillment \\
of the Requirements for the Degree \\
Doctor of Philosophy \\
\hline Modeste Tchakoua Tchouaso \\
br. Mark A. Prelas, Dissertation Supervisor
\end{tabular}

DECEMBER 2017 
The undersigned, appointed by the dean of the Graduate School, have examined the thesis entitled

\section{AN INVESTIGATIVE STUDY ON NEUTRON EMISSION FROM TITANIUM DEUTERIUM SYSTEM UNDER THERMAL SHOCK}

presented by Modeste Tchakoua Tchouaso

a candidate for the degree of Doctor of Philosophy

and hereby certify that, in their opinion, it is worthy of acceptance.

Professor Mark A. Prelas

Professor Sudarshan K. Loyalka

Professor Tushar K. Ghosh

Professor William H. Miller

Professor Tompson, Jr., Robert V

Professor Stephen Montgomery-Smith 


\section{ACKNOWLEDGEMENT}

I would like to express my deepest gratitude to Professor Mark A. Prelas for giving me an opportunity to work on this project. His trust, guidance, and encouragement led to the success of this work.

I would like to thank my thesis committee members, Dr. Sudarshan Loyalka, Dr. Tushar Ghosh, Dr. Robert Tompson, Dr. William H. Miller, and Dr. Montgomery-Smith for their guidance and generosity.

I am indebted to the Fulbright scholarship program, the Nuclear Science and Engineering Institute, and the Sidney Kimmel Institute for Nuclear Renaissance for their financial support. I am very grateful to my father, Tchouaso Jeremiah, my mom, Ndengoue Anne, and my siblings for their support, encouragement and love throughout the years.

I am truly grateful to the staff of the Nuclear Engineering and Engineering Institute, Latricia Vaughn and James Bennett for their support throughout the years.

I would also like to thank Dr. Charles Weaver and my laboratory partner Dr. Haruetai Kasiwattanawut, my friends and classmates for their support throughout these years. 


\section{TABLE OF CONTENT}

ACKNOWLEDGEMENT ...............................................................................

LIST OF ILLUSTRATIONS ...........................................................................

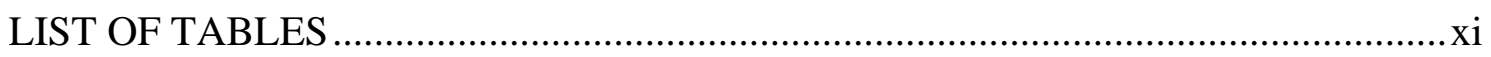

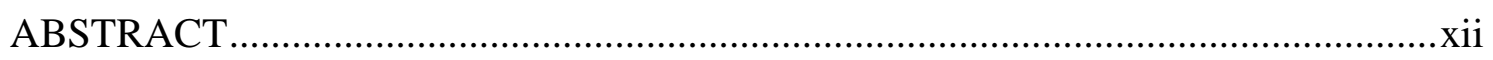

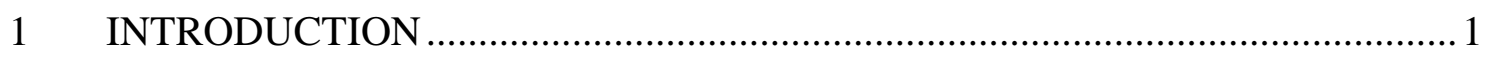

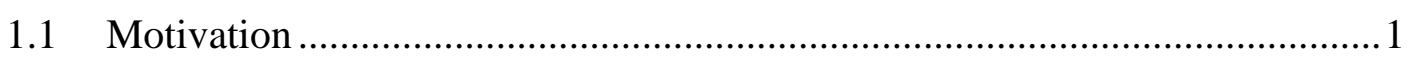

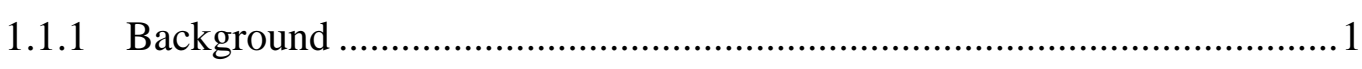

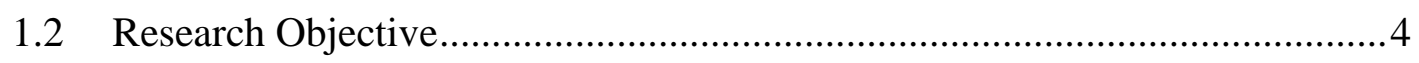

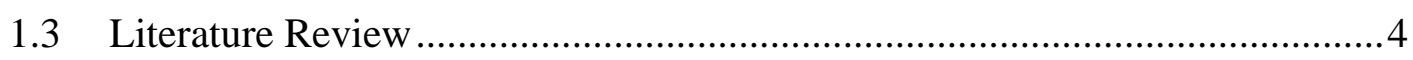

1.3.1 Interaction of Deuterium with Titanium .............................................

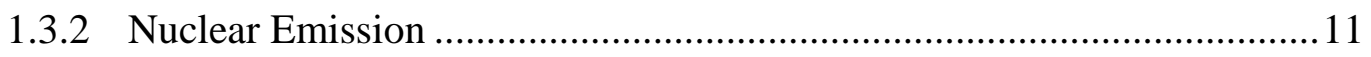

1.3.3 Helium-4 and Tritium Production ......................................................... 17

1.3.4 Theories and Mechanism on Neutron Production...................................22

1.3.5 "Phase Transition Mechanism" ..............................................................22

1.3.6 Fracto-fusion Mechanism...................................................................2

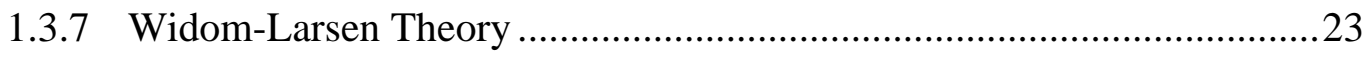

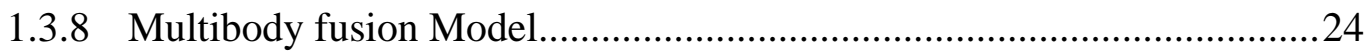

1.3.9 Trapped Neutron Catalyzed Model for Cold Fusion (TNCF Model) ......24 


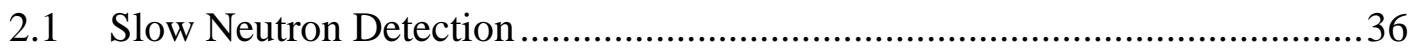

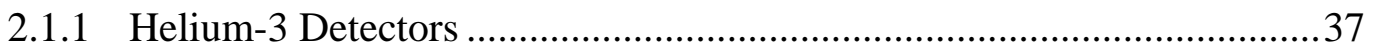

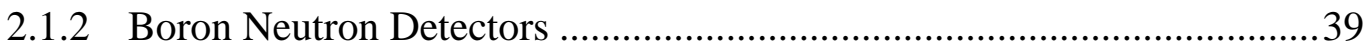

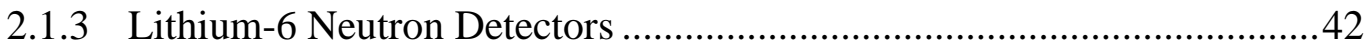

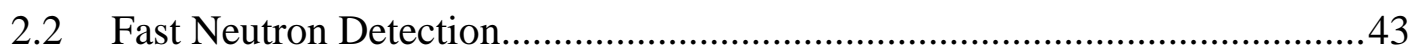

2.2.1 Neutron Scintillation Detection........................................................45

2.2.2 Proton Recoil Proportional Counter ....................................................46

2.2.3 Neutron Unfolding ................................................................... 48

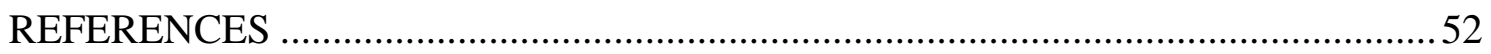

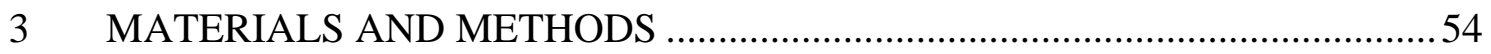

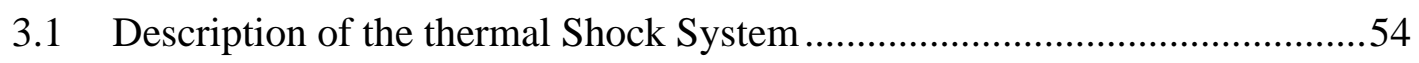

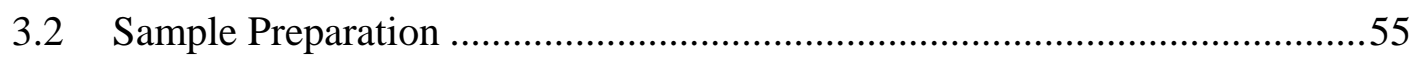

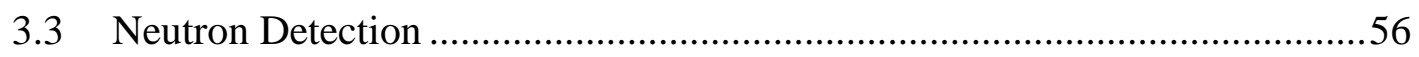

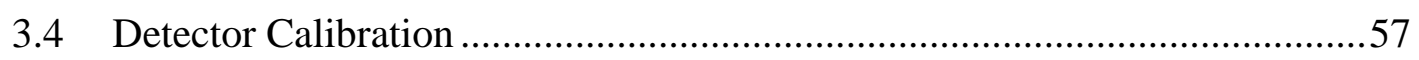

3.5 X-ray Diffraction Measurements .............................................................63

3.6 Scanning Electron Microscope and Energy Dispersive Microscopy ..............65

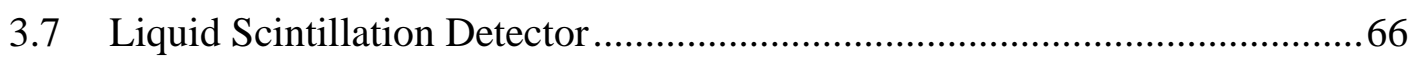

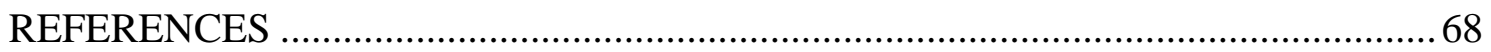




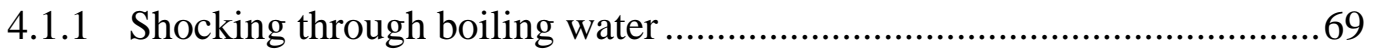

4.1.2 Shocking by means of a Heat Gun .................................................... 90

4.1.3 Liquid Scintillation Detector Analysis ................................................109

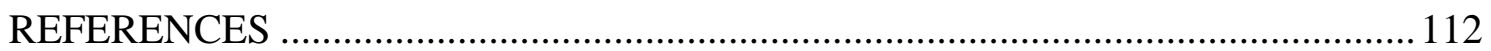

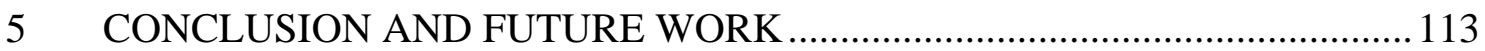

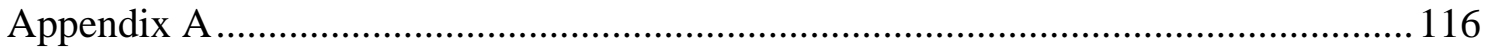

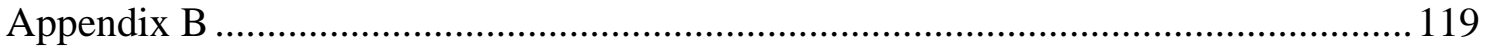

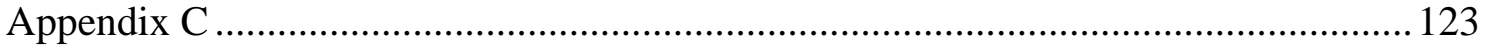

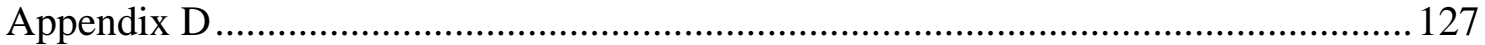

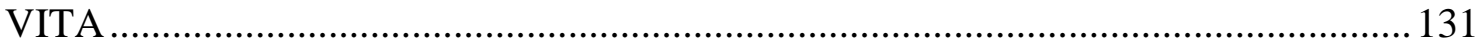




\section{LIST OF ILLUSTRATIONS}

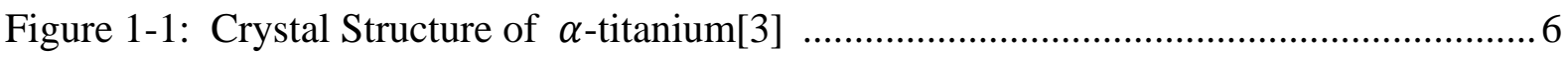

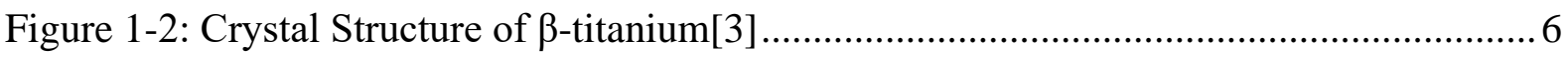

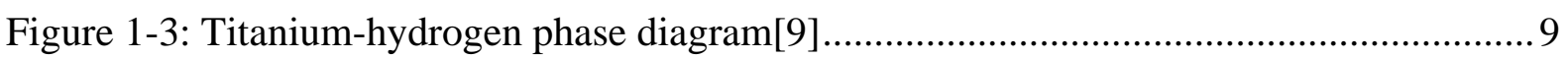

Figure 2-1: Neutron cross section for various materials used in neutron detectors [3] ............32

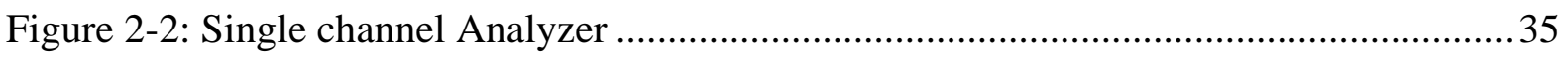

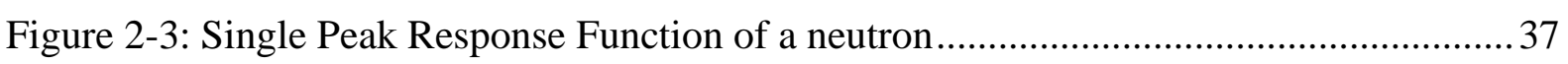

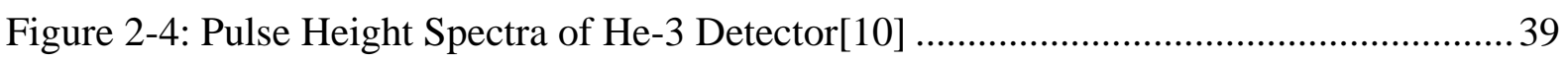

Figure 2-5: Expected pulse height spectrum from $\mathrm{BF}_{3}$ detector ..................................... 41

Figure 2-6: Pulse Height Discrimination of fast neutrons on EJ309 detector from PuBe ....... 46

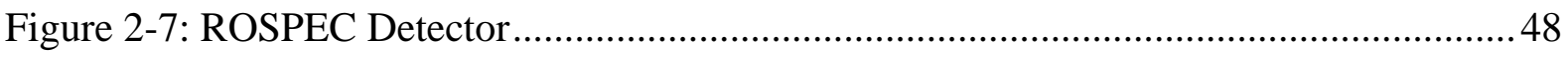

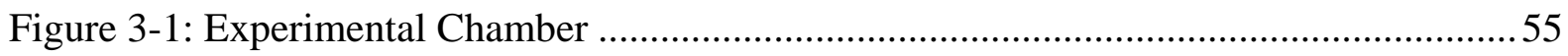

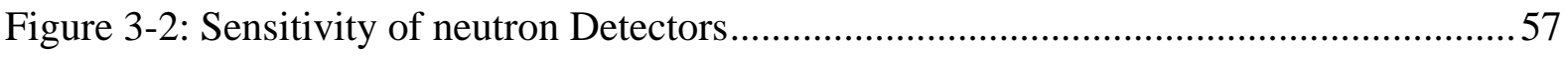

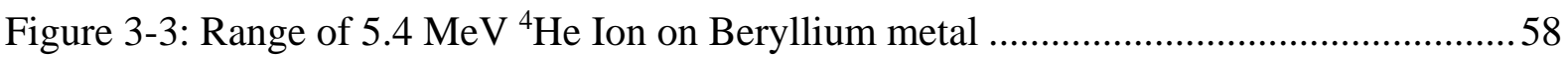

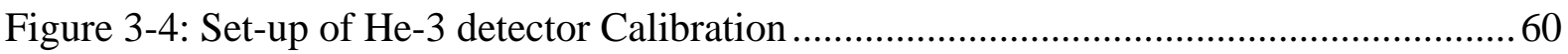

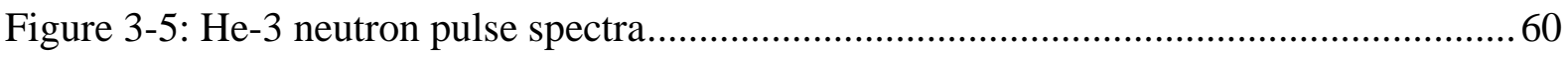

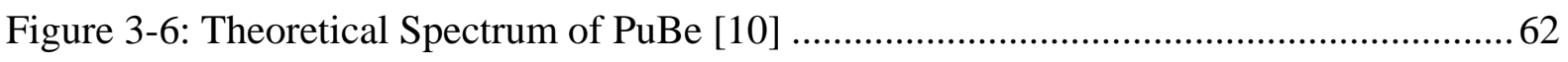

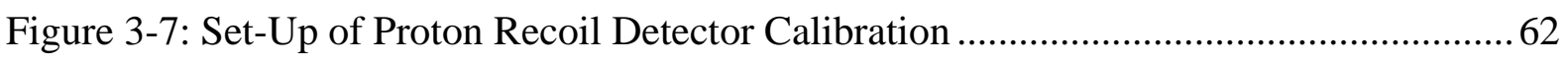

Figure 3-8: Raw Proton Recoil spectra of PuBe Source .................................................. 62

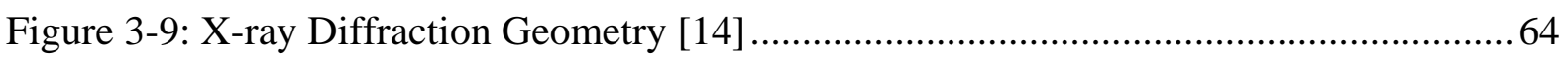

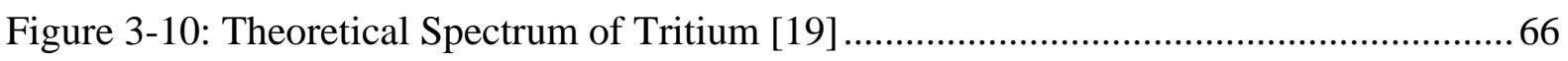

Figure 4-1: EDS spectra of titanium powder used in Experiment 1 .................................... 71

Figure 4-2: Moderated He Detector-Background Coun ..................................................... 72

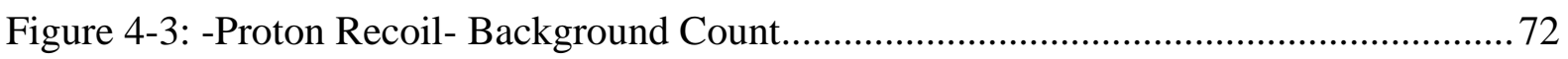

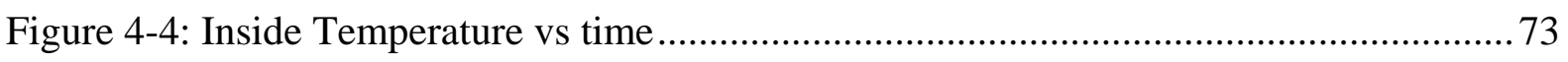

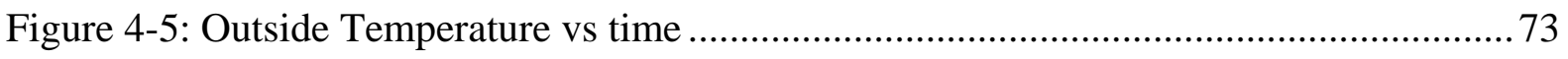


Figure 4-6: Pressure Variation over time....

Figure 4-7: Moderated He-3- Foreground Count Rate …................................................. 73

Figure 4-8: Foreground Count in Proton Recoil Detector .................................................... 74

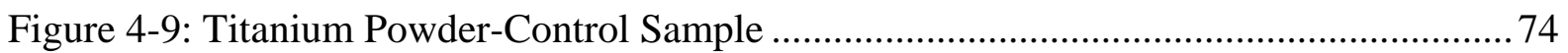

Figure 4-10: Titanium powder - Experimental Sample ..................................................... 74

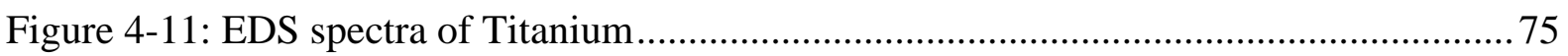

Figure 4-12: moderated ${ }^{3} \mathrm{He}$-Background Count Rate...................................................... 76

Figure 4-13: Unmoderated ${ }^{3} \mathrm{He}$ Background Count Rate .................................................. 76

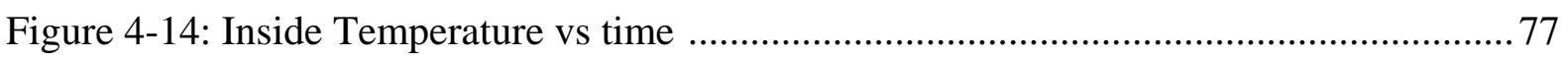

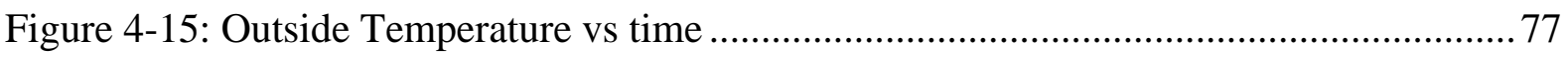

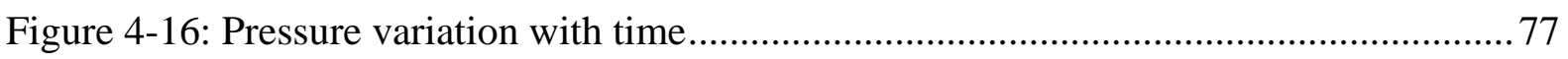

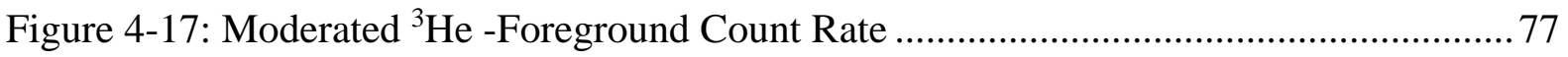

Figure 4-18: Unmoderated ${ }^{3} \mathrm{He}-3$-Foreground Count Rate ................................................. 77

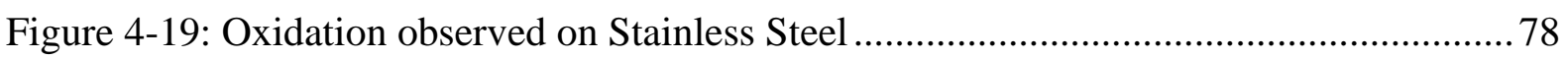

Figure 4-20 : Huge Temperature Observed in the titanium - deuterium System.................... 79

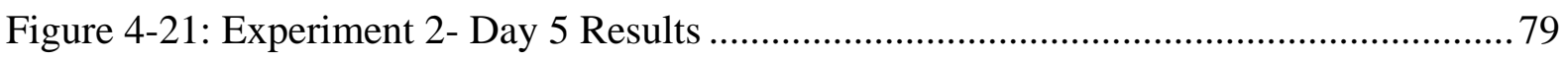

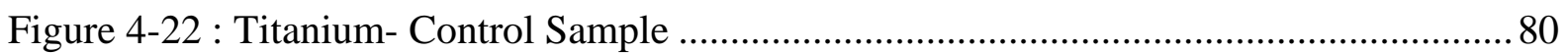

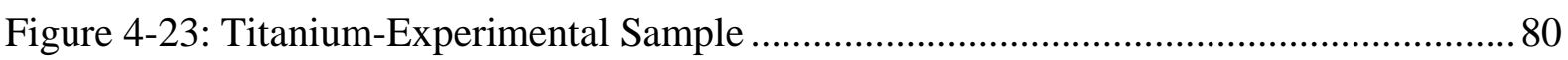

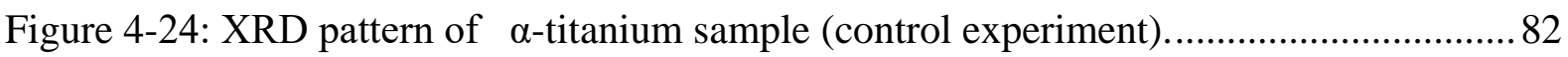

Figure 4-25: X-ray Diffraction (XRD) pattern showing titanium deuteride formation........... 82

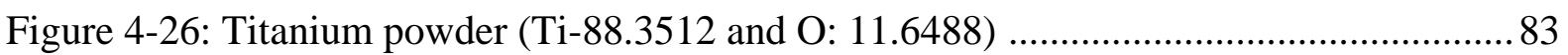

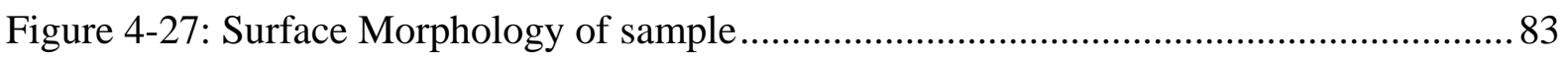

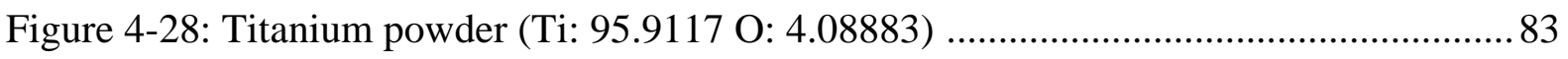

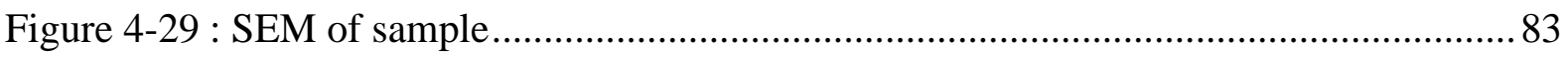

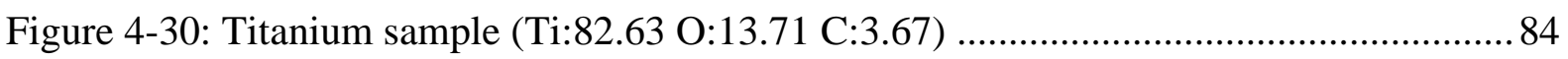

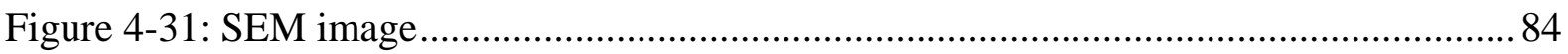


Figure 4-32: Titanium composition in Titanium powder .85

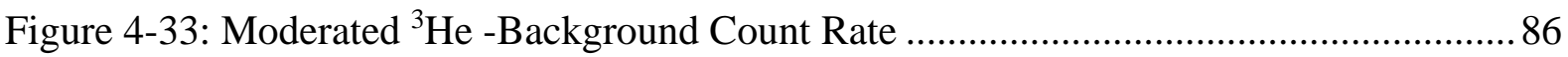

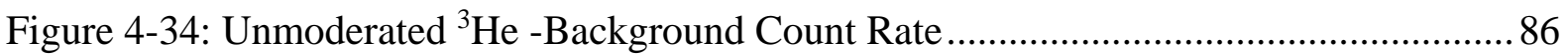

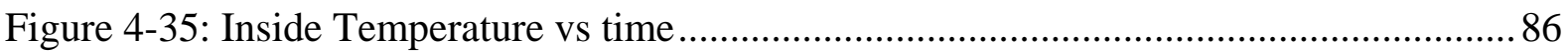

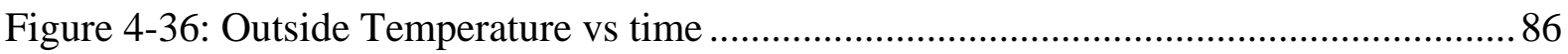

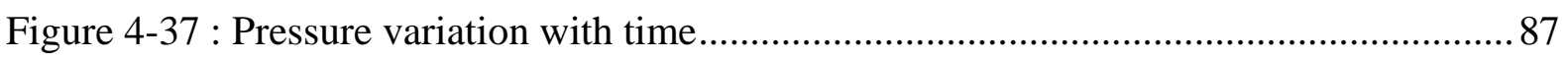

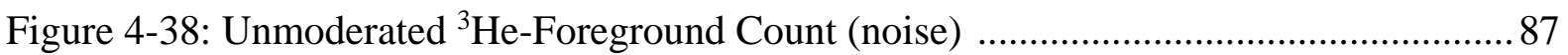

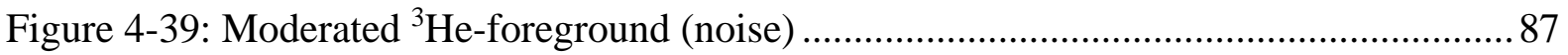

Figure 4-40: Observed count rate from Cf-252 - unmoderated Helium-3 detector ................. 88

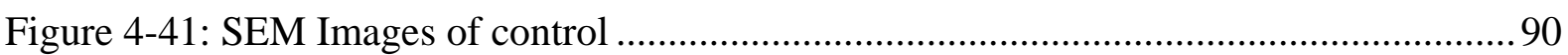

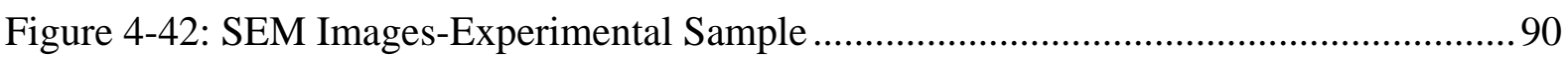

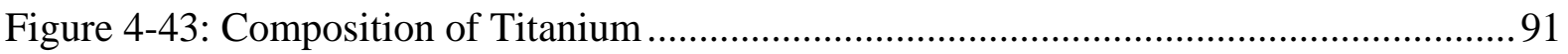

Figure 4-44: Moderated He3 -Background Count ................................................................ 91

Figure 4-45: Unmoderated He3 -Background Count......................................................... 91

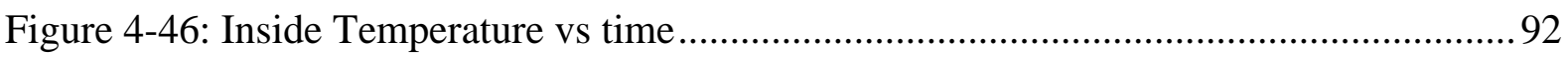

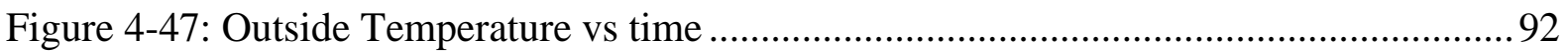

Figure 4-48: Pressure Variation with time during and after shocking ..................................92

Figure 4-49: Moderate ${ }^{3} \mathrm{He}$ - Foreground Count............................................................... 92

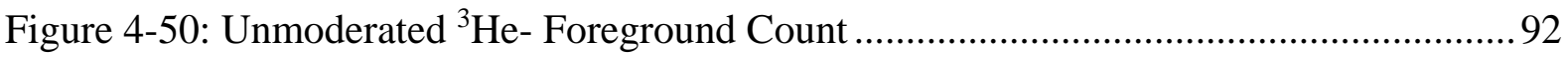

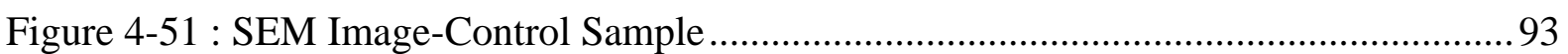

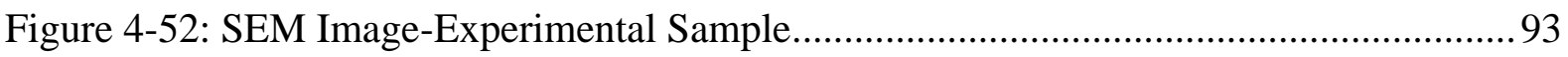

Figure 4-53: Composition of titanium used in thermal shock experiment ............................94

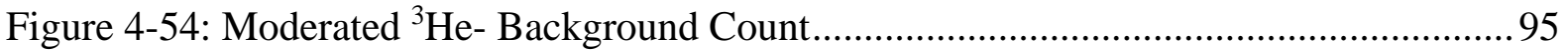

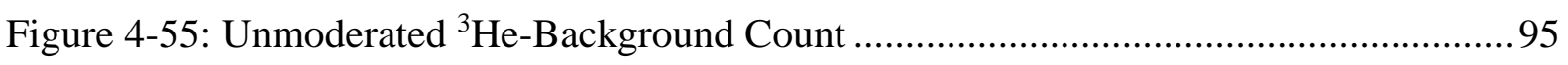

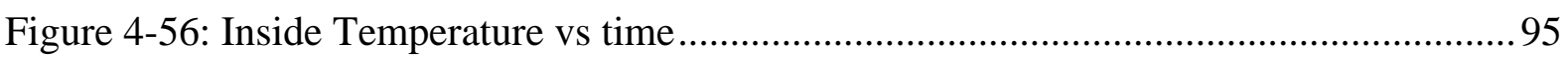

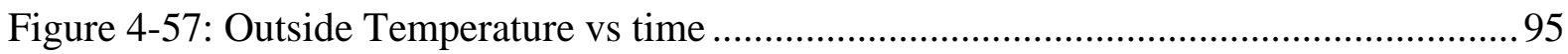


Figure 4-58: Pressure drop during loading .95

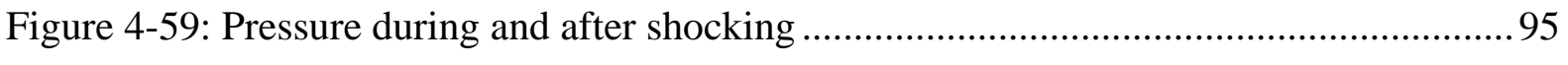

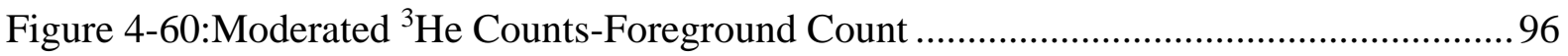

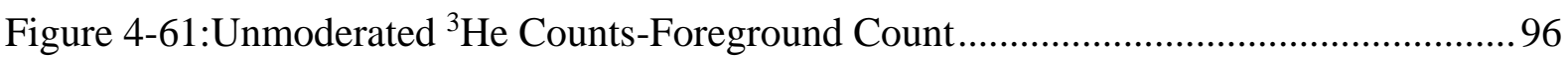

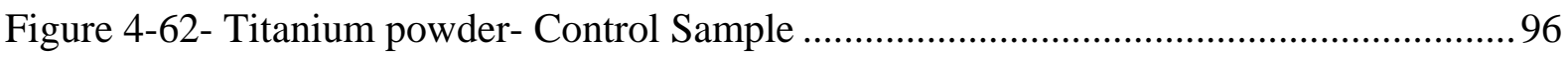

Figure 4-63: Titanium powder- Experimental sample..................................................... 96

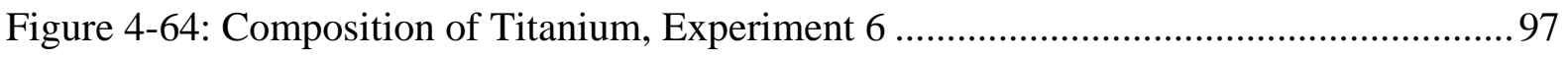

Figure 4-65:Moderated ${ }^{3} \mathrm{He}$ Detector-Background Count ................................................ 98

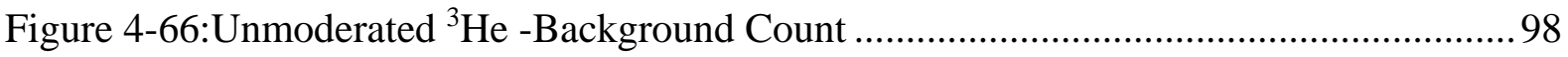

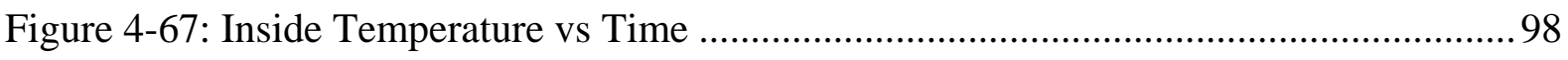

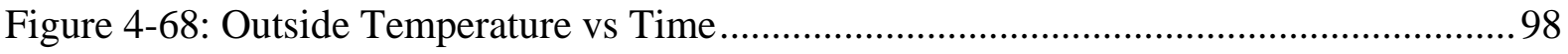

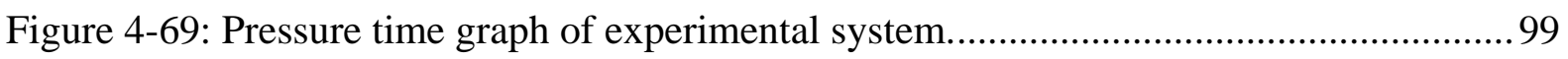

Figure 4-70: Moderated ${ }^{3} \mathrm{He}$ Detector-Foreground Count ................................................ 99

Figure 4-71: Unmoderated ${ }^{3}$ He Detector-Foreground Count ........................................... 99

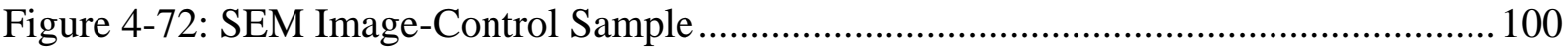

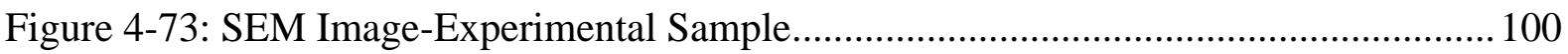

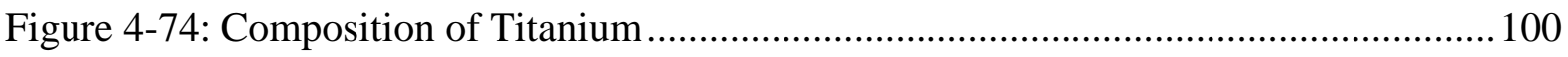

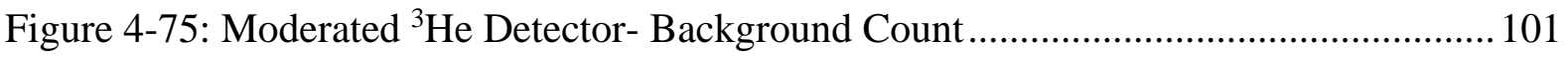

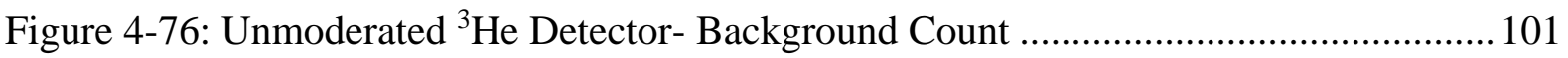

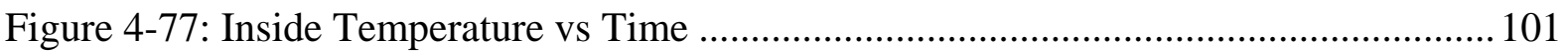

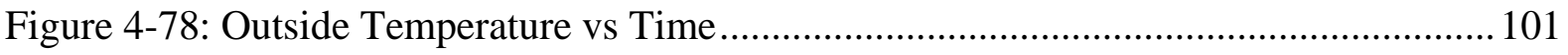

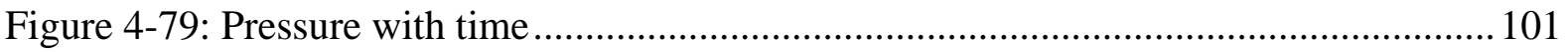

Figure 4-80: Moderated ${ }^{3}$ He Detector- Foreground Count................................................. 102

Figure 4-81: Unmoderated ${ }^{3}$ He Detector- Foreground Count .......................................... 102

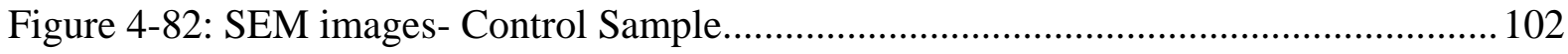

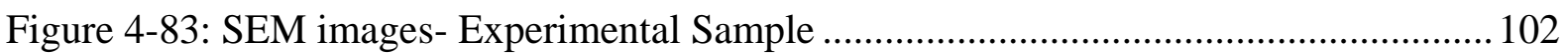


Figure 4-84: Composition of Titanium 8

Figure 4-85: Moderated ${ }^{3}$ He Detector- Background Count ................................................... 103

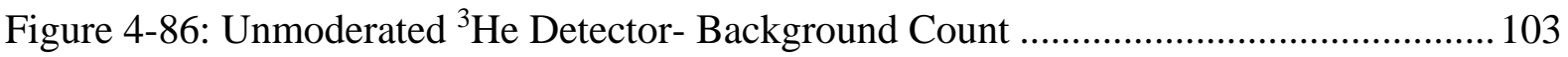

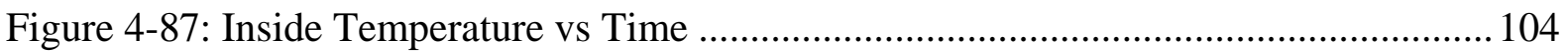

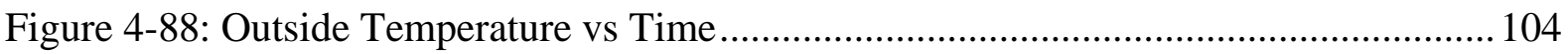

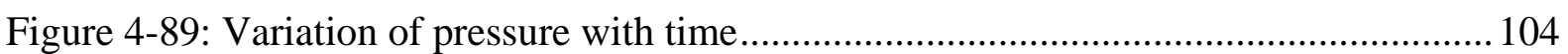

Figure 4-90: Moderated He3 Detector- Foreground Count ................................................ 104

Figure 4-91: Unmoderated He3 Detector- Foreground Count............................................ 104

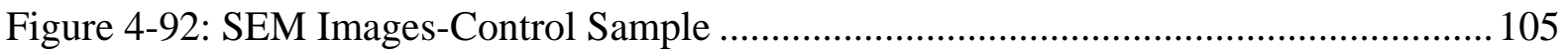

Figure 4-93: SEM Images-Experimental Sample ….................................................... 105

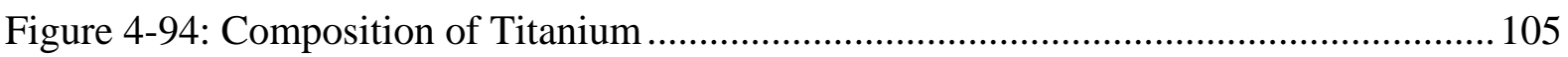

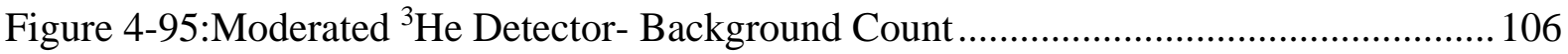

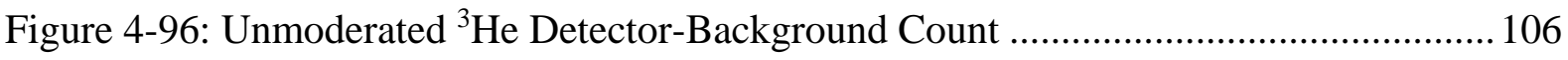

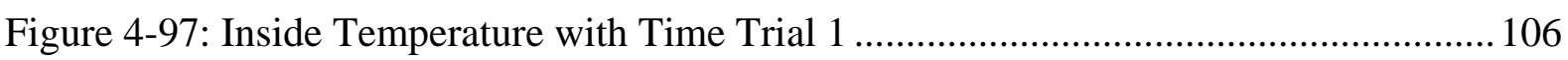

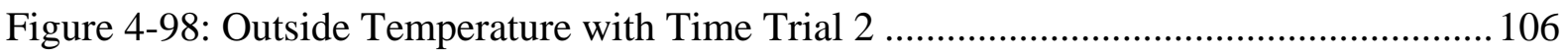

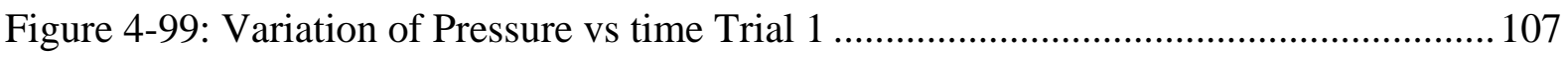

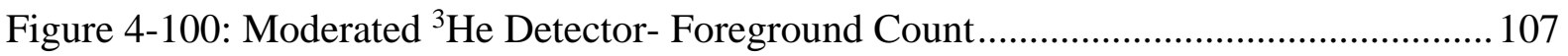

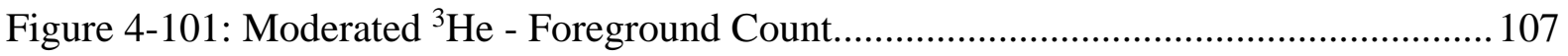

Figure 4-102: Temperature change-first trial outside(right) (Inside left) Trial 2 ................. 107

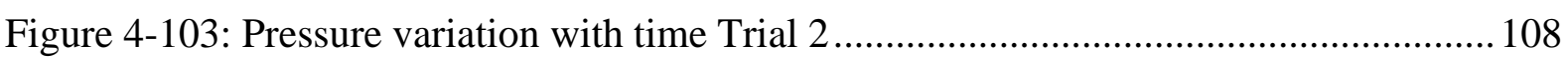

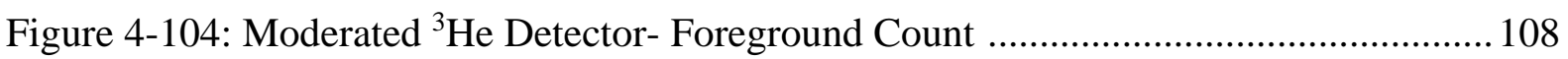

Figure 4-105: Unmoderated ${ }^{3} \mathrm{He}$ Detector- Foreground Count ........................................ 108

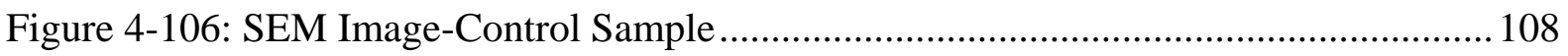

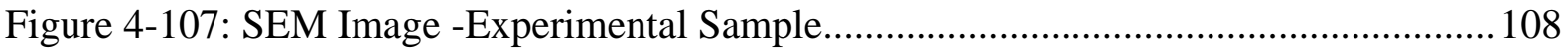




\section{LIST OF TABLES}

Table 1-1: Mechanism of solid phase transformation in Titanium Hydride (TiHx) [6] ........... 9

Table 1-2: Heat of Dissociation of Titanium Deuteride ...................................................... 10

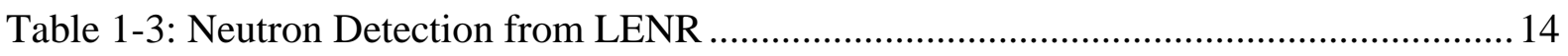

Table 1-4: Tritium Measurements in Different Systems ................................................... 19

Table 1-5: Detection Environment used in Table 1-5 ......................................................... 20

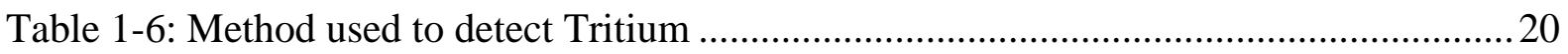

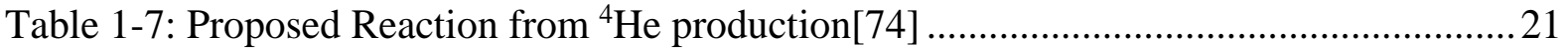

Table 2-1: Gamma Ray Interaction Probability for Different Detectors[4] .......................... 34

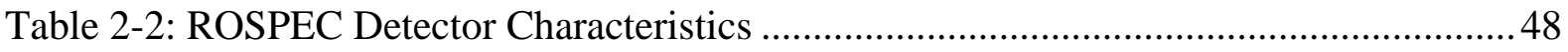

Table 4-1: Composition of Titanium in Experiment 1....................................................... 71

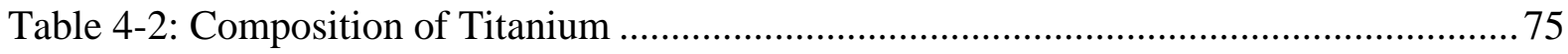

Table 4-3: Composition of Titanium used in the Experiment 3 ........................................ 85

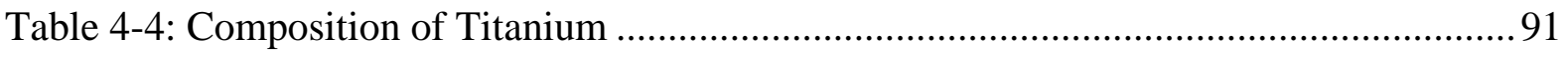

Table 4-5: Composition of Titanium Powder ................................................................... 94

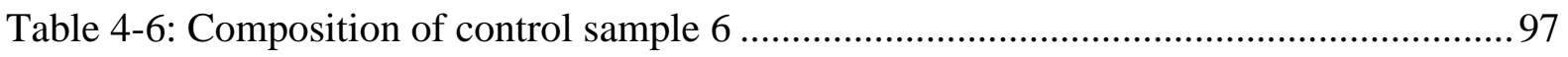

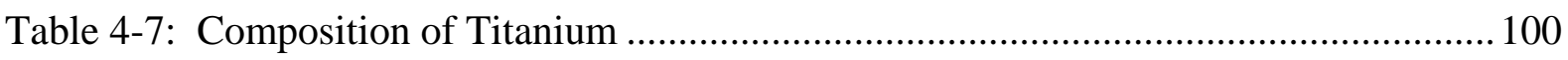

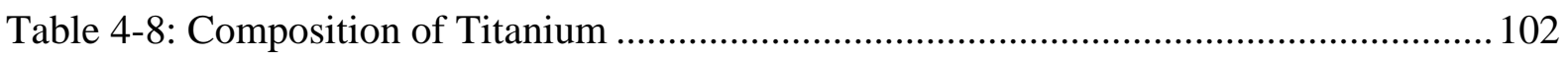

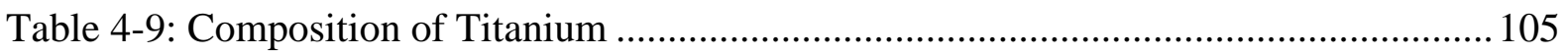

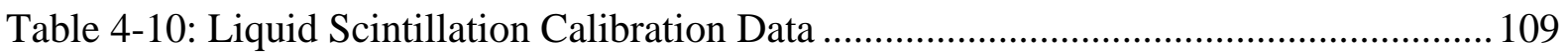

Table 4-11: Summary of Background count rate of LSC …............................................. 110

Table 4-12: Summary of LSC results from Titanium-deuterium loading ........................... 110 


\begin{abstract}
The purpose of this study is to investigate the titanium-deuterium system under thermal shock, as a potential neutron source. The expected neutron emission is unique, i.e. it is monoenergetic with energy of $2.45 \mathrm{MeV}$, which is valuable for calibrating neutron detectors. In our study, titanium was loaded with deuterium gas at room temperature in an experimental system, and the system was subjected to rapid thermal cycling by repeated cooling with liquid nitrogen, followed by rapid warm up phases to create a non-equilibrium condition in titanium lattice. Neutron bursts were monitored using a ${ }^{3} \mathrm{He}$ detector, which responds to slow neutrons, a moderated ${ }^{3} \mathrm{He}$ detector, which responds to slow and fast neutrons, and a proton recoil detector, which responds to fast neutrons. The pressure and temperature of the system was monitored throughout the experiments. The result of this work shows that: 1) loading of titanium with deuterium gas should be done under high vacuum conditions $\left(<1 \times 10^{6}\right.$ torr $)$ to remove environmental contaminants, which was found to inhibit the titanium-deuterium reaction, 2) cracks observed in titanium samples from lattice stress varied in size and location in titanium lattice and dependent on the level of deuterium loading. The presence of cracks in some locations indicates that the titanium-deuterium reaction is a local effect, 3) low level neutron burst were observed in less than $23 \%$ of all experiments and involved the detection of a single neutron burst, suggesting that neutron emission is a statistical process occurring at low probability. The neutron burst was observed from partially deuterated titanium samples. The level of neutrons detected is consistent with what has been reported in literature. 4). A large temperature increased from room temperature to $450{ }^{\circ} \mathrm{C}$ during phase transition from $\alpha$ titanium to $\delta$-titanium occurred, but no neutrons were observed. The temperature increased is likely associated with the exothermic reaction that occurs during hydride formation, which does not lead to neutron emission. 5) No evidence of tritium or nuclear transmutation was observed in our experimental system.
\end{abstract}




\section{INTRODUCTION}

\subsection{Motivation}

Neutron emission from titanium-deuterium systems is of great interest because of the nature of the neutron expected from the titanium-deuterium reaction. The expected neutrons emission is unique, i.e. it is monoenergetic with energy of $2.45 \mathrm{MeV}$ which is useful for calibration of neutron detectors. The mechanism through which these neutrons are produced is not well established, and there is the issue of irreproducibility of the observed neutron emissions. Understanding the nature of the neutrons produced is challenging since it requires investigations on the role of various components of the system. This include investigating the role of surface conditions, phase transitions, and crack formation or stress in titanium lattice during titanium deuteride formation. This information is not found in literature which makes it difficult to explain the observed nuclear events. Methods such as the electrolytic, gas-loading, electrochemical, and gas discharge method have been used to create the necessary condition needed in condense matter to induce a nuclear effect. These methods are based on changing the thermodynamic conditions of condense matter systems by changing the pressure or temperature or both. The gas-loading technique is the preferred method of choice for our investigation because it is easy to get rid of environmental contamination such as water vapour and oxygen from the experimental system and the pressure or temperature of the system can be varied with ease when using this method. The experimental samples can also be obtained at the end of the experiment for analysis to determine any morphological changes that occurs during the titanium-deuterium reaction.

\subsubsection{Background}

Research has been undertaken at the University of Missouri since 1991 to understand the phenomena of nuclear emissions from titanium-deuterium systems. The research was 
initiated by reports of nuclear effects reported in 1989[1] and later by Jones and coworkers [2]. The observed effect was that of heat production and nuclear radiation, involving a unique mechanism occurring at low temperatures. The motivation for the studies of heat production stems from the belief that it could provide a clean, and cheap source of commercial energy. The expected nuclear products include; neutrons, gamma radiation, $\mathrm{x}$-rays, beta radiation, alpha radiation and proton. The charge particles (proton, tritium, alphas and beta radiation) expected from this reaction has a short range in most material and will not penetrate the structural material used in most experimental systems. Detection of charge particles would require charge particle detectors to be placed inside the experimental system, which is often difficult. Neutrons on the other hand, can easily penetrate experimental systems since they have a long range in matter. The expected neutron radiation is monoenergetic, which makes it particularly useful for calibration of neutron detectors. Therefore, many research studies have focused on detecting the $2.45 \mathrm{MeV}$ neutrons.

Neutron emissions from induced nuclear effect due to change in thermodynamic conditions of titanium-deuterium or palladium-deuterium systems have been investigated, and several authors have reported neutron production from their experimental systems [1-4]. Despite reports of neutron emissions, an important challenge has been that of reproducible of these results. Not all investigations have confirmed neutron emissions (there is no doubt that neutrons can be produced from d-d fusion reaction at moderately low temperatures as has been demonstrated by the Inertial Electrostatic Confinement (IEC) fusion. IEC devices have found commercial applications in oil well logging, as low-level neutron sources and as calibration sources for neutron detectors in many facilities around the world).

The titanium-deuterium system came from studies trying to produce low energy nuclear reaction. However, the mechanism for neutron production is most likely not related to the classic low energy fusion experiment. As previously stated, the neutron production 
mechanism is most likely related to classic fusion - its postulated mechanism is fracto-fusion where very high electrostatic fields are created when the metal lattice of titanium cracks. The inconsistency in neutron production observations in the titanium-deuterium system has been attributed to be from the fact that the active region where fusion reaction occurs is tiny and occurs at random locations. The neutron production is therefore a local fusion process that occurs at a certain location within condense matter and not in others (i.e., the unpredictability of crack formations). The number of active sites where the local fusion occurs can be enhanced by increasing the number of collisions between the gas and target material, increasing the surface area of the solid material, surface treatment of the sample, creating defects in the sample, and changing the thermodynamic condition of the titanium-deuterium system. It is important to understand how these different factors affect the rate of neutron production in these systems. Such investigations would provide valuable information that can enhance reproducibility. Another challenge with this system is that there is no useful theory to guide the research and most success is usually achieved by chance. The statistical nature and the lack of satisfactory theories makes it particularly difficult to achieve success in experiments. Useful theories can only be made based on the results of experimental work. It is the hope of this work therefore to produce some experimental results that can help shape existing theories.

Several methods have been used to initiate the fusion reaction in condense matter systems. There are classical methods such as magnetic confinement (tokomaks), gravitation confinement (sun), inertial confinement (laser fusion), electrostatic confinement (IEC), beam interactions with targets (Cockcroft-Walton Generators), etc. There are also claims that low energy nuclear reactions can produce fusion through electrochemical[5-9], electrical discharge[10, 11], and gas loading[1] techniques. While the original reports used an electrochemical means to generate energy, this method generally introduces hydrogen into 
the experimental system. It is possible to introduce deuterium directly into transition metals without introducing hydrogen. The gas loading method is an excellent method of achieving this but there is no evidence that the reaction is based on fusion. The method can be used to analyze microscopic properties of samples because of the simplicity of its system compared to the electrolytic method. The gas loading technique offers other benefits as well:

1. Environmental contaminations such as oxygen, which forms oxides when it interacts with the titanium surface, thereby occupy sites in titanium lattice can be removed using advanced vacuum systems;

2. The titanium powder stays in bulk

3. The deuterium gas can be collected at the end of the experiment for analysis.

4. It is much simpler than the electrolytic method.

5. It is easy to change the thermodynamic condition of the system, i.e. change in temperature or pressure.

The titanium-deuterium system studied here is based on gas loading techniques.

\subsection{Research Objective}

This thesis is aimed at (1) determining the mechanism of neutron emission from titanium-deuterium systems under thermal shock, (2) investigating the role of crack formation on neutron production, (3) investigating the relationship between heat production and neutron production, and, 4) determining level of tritium production from the low energy nuclear reaction, and 5) determining if nuclear transmutation occurs in titanium-deuterium systems.

\subsection{Literature Review}

\subsubsection{Interaction of Deuterium with Titanium}

The behavior of isotopes of hydrogen (the chemical property of hydrogen and deuterium are similar ) in transitions metal has been widely investigated because of the interest in using transition metals as a hydrogen storage material. These studies have provided significant details on the thermodynamic behavior of metal hydrides, which is significant in determining the mechanism of energy production and nuclear emissions from condense 
matter. Many transition metals are exothermic absorbers of hydrogen forming hydride phases. Titanium, nickel and palladium are among the metals widely used in gas loading studies because of their ability to hold huge amount of hydrogen isotopes. Titanium has been the second metal of choice after palladium for used in electrolytic experiments. Palladium is known to absorb huge amounts of hydrogen or deuterium into the bulk of the material where hydrogen is inserted, occupy interstitial octahedral or tetrahedral sites depending on the specific palladium-hydride phase formed. Hydrogen has been used in most investigations involving palladium as a host metal, while titanium has been used mostly with deuterium. Unlike palladium, titanium is cheap and is the choice of material in some investigations. For this reason, it was used in our investigation. Our discussion will therefore be limited to titanium.

An important property of pure titanium, which makes it useful for gas loading studies is that pure titanium is resistant to corrosion because of a passive oxide film that makes it resistant to corrosion in oxidizing solutions [1]. This property of titanium is highly desired as environmental contamination limits the rate of hydrogen absorption. Titanium is an allotropic element, existing in one or more forms. At ambient temperature and pressure, it has a hexagonal closed-packed (hcp) crystal structure with a ratio of its lattice parameter given by $\frac{c}{a}=1.587$. This structure is known as the $\alpha$-titanium. The insertion of interstitial atoms in the hcp lattice slightly increases the ${ }^{c} / a$ ratio. At a temperature of $882 \pm 2{ }^{0} \mathrm{C}$, the bodycentered cubic structure known as $\beta$-titanium $(\beta$-Ti) is more stable. The lattice parameter of $\beta$-Ti is $a=0.332 \mathrm{~nm}$. The level of absorption that occurs in $\alpha$-Ti and $\beta$-Ti is influenced by their lattice structure. The hexagonal closed packed atom in $\alpha$-Ti causes its diffusion coefficient to be an order of magnitude smaller than that of $\beta$-Ti at $1000{ }^{\circ} \mathrm{C}, D_{\alpha-T i} \approx$ $10^{-15} \mathrm{~m}^{2} / \mathrm{s}$ while $\left.D_{\beta-T i} \approx 10^{-13}\right)$. The diffusion length after one hour is about $4 \mu \mathrm{m}$ and $40 \mu \mathrm{m}$ for $\beta$-Ti and $\alpha$-Ti, respectively[2], which is about ten times less in $\beta$-Ti. The 
difference is due to the microstructure of the two different phases. The $\beta$-Ti is therefore an interesting candidate in achieving large concentration of hydrogen or deuterium. Figure 1-1 and Figure 1-2 below show the crystal structure $\alpha$-Ti and $\beta$-Ti.

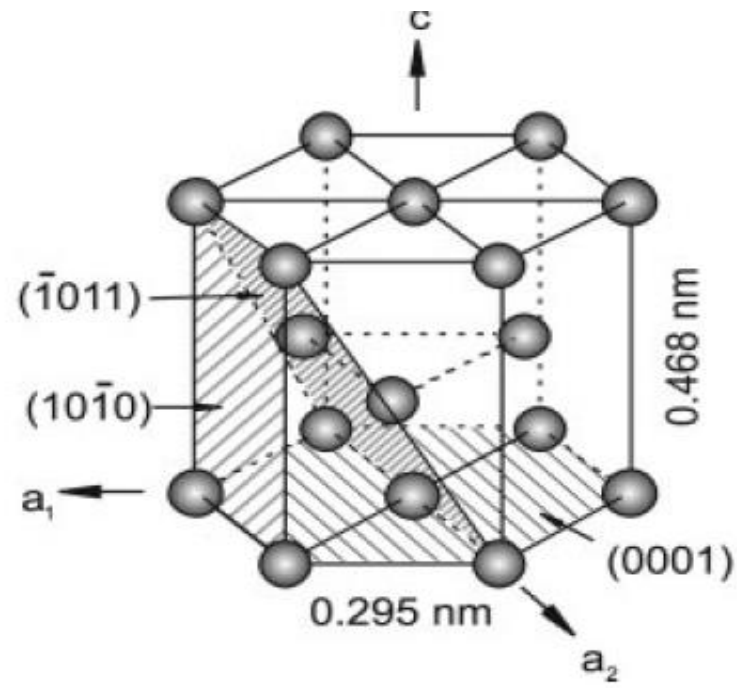

Figure 1-1: Crystal Structure of $\alpha$-titanium[3]

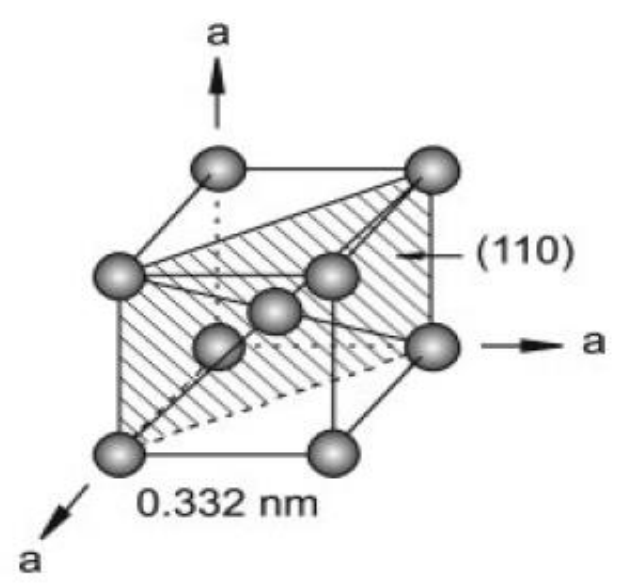

Figure 1-2: Crystal Structure of $\beta$-titanium[3]

Titanium has received considerable attention as a hydrogen storage material since it possesses a high affinity for hydrogen isotopes. As a result, the titanium-hydrogen system has been studied extensively. The absorption of hydrogen in titanium is influenced by the supplied gas pressure, activation temperature, the cleanliness of the system, and thermal degassing of titanium[4]. The degassing process is the most efficient because it leads to rapid absorption at room temperature[5]. Titanium-hydrogen system is particularly interesting because it is a reversible system[6]. This property has been exploited to produce neutron emissions in titanium- deuterium systems. Titanium undergoes hydrogen embrittlement due to lattice stress induced in the material [7] from absorption of hydrogen. Hydrogen embrittlement is a great concern when titanium alloys are used in nuclear reactors and aircraft. Neutron production in the deuterium-titanium system is believed to occur from stress induced in titanium lattice from hydrogen absorption. Structural transformation occurs in 
titanium crystal lattice from increase in hydrogen concentration. Changes in crystal structures results in volume changes and redistribution of hydrogen atoms in the interstitial sites of the crystal[8].

The behavior of titanium can be represented in terms of phase diagrams. The phase diagram for titanium-hydrogen system at $P \leq 1 M P a$ is shown in Figure 1-3. The type of hydride formed depends on the path taken. At the lower region of the phase diagram, i.e. for the lower concentration, the solid solubility of hydrogen in $\alpha$-phase is very small at room temperature. The $\alpha$-phase has a hexagonal closed-packed (hcp) structure and is stable at TiH $_{x}$ with $x(x \leq 0.1)$. At a higher concentration of hydrogen, $x(0.1 \leq x \leq 0.44)$, this phase gives way to a body-centered cubic (bcc) structure called the $\beta$-phase. The $\beta$-phase has a higher solubility than the $\alpha$-phase and is therefore less vulnerable to embrittlement. The temperature of the $\alpha \leftrightharpoons \beta$ transformation is reduced by $600^{\circ} \mathrm{C}$ due to an increase in hydrogen content. Further increased in hydrogen concentration, $x(0.44 \leq x \leq 0.5)$ leads to the $\gamma$ phase[8]. The $\gamma$-phase is face-centered tetragonal (fct) lattice with $c>a$. This phase is followed by the $\delta$-phase, $x(0.5 \leq x \leq 0.66$, which has a face-centered cubic lattice (f.c.c), and finally by the $\varepsilon$-phase $x(0.66 \leq x \leq 0.67)$ with face-centered tetragonal (fct) lattice[9]. The transition from $\gamma \rightarrow \delta$ occurs at a temperature of $673 \mathrm{~K}$ while the transition from $\delta \rightarrow \varepsilon$ occurs at a temperature of $310 \mathrm{~K}[10]$. At a temperature of $573 \mathrm{~K}$, the $\beta$-solid hydrogen solution dissociate into the $\alpha$-solid solution and the $\delta$-hydride through the eutectoid reaction[11]. A summary of these phases is shown in Table 1-1. Mixed phases are said to occur within $x(0 \leq x \leq 0.67)$. Wedler and Co-workers have shown that there is an $\alpha-$ $\alpha+\delta$ boundary at 6-at. $\% \mathrm{H}$, and an $\alpha+\delta-\delta$ boundary at 40-at. $\% \mathrm{H} \mathrm{[12]} \mathrm{and}$ McQuillan reported that the $\beta$-phase is preceded by $(\alpha+\beta)$ phase and followed by the $(\beta+$ $\gamma)$ mixed phase [13]. 
The location of hydrogen atoms in titanium-deuterium systems is important in determining the type of reaction that can occur. Hydrogen atoms can be located in tetrahedral T-interstitials and in the Octahedral, O-interstitials sites in titanium. In most cases, not all possible sites are filled with hydrogen atoms leaving vacancy in the crystal. The hydrogen atoms are therefore free to move from one site to another. The hcp structure has 2 octahedral and 4 tetrahedral interstitial sites. There is no consensus on the location of hydrogen in $\alpha$ phase lattice. However, most researchers believe that hydrogen is located in the tetrahedral sites. The bcc structure has 12 tetrahedral and 6 octahedral sites. Neutron diffraction data has shown that deuterium atoms occupy the tetrahedral sites. Investigations on the phase transition that occurs leading to the $\beta$-phase shows a net increase of about $6 \%$ in the volume of the $\beta$-phase from the pure $\beta$-phase[11]. The $\delta$-phase exist when the concentration of hydrogen in titanium-hydrogen system is $(1.5 \leq x \leq 2)$, where $x$ is the number of hydrogen atoms per titanium atom. In the temperature range of $196^{\circ} \mathrm{C}$ to $200^{\circ} \mathrm{C}$, the hydride phase is formed with an fcc metal lattice and the hydrogen atoms randomly occupying the tetrahedral interstitial sites. Since the diffusion rate is directly proportional to the number of unfilled tetrahedral sites, the diffusion of hydrogen takes place via a vacancy mechanism. X-ray diffraction analysis has shown that a tetragonal distortion occurs at this region [14]. The unit cell of the $\gamma$-phase contains four titanium atoms and four hydrogen atoms and corresponds to the formula $\mathrm{TiH}$. 


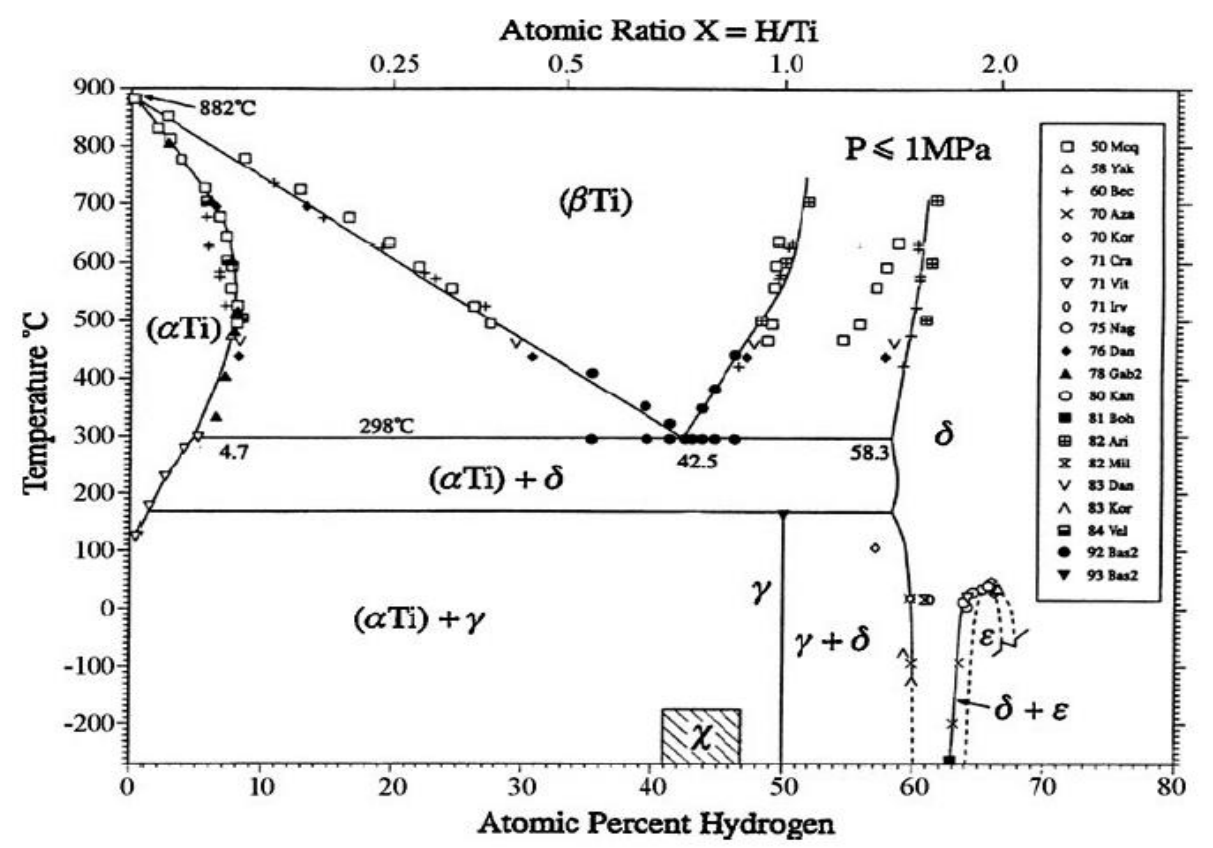

Figure 1-3: Titanium-hydrogen phase diagram[9]

Table 1-1: Mechanism of solid phase transformation in Titanium Hydride (TiHx) [6]

\begin{tabular}{|l|c|c|c|c|c|}
\hline Phase & \multicolumn{1}{|c|}{$\boldsymbol{\beta}$} & $\boldsymbol{\beta}$ & $\boldsymbol{\delta}$ & $\boldsymbol{\varepsilon}$ \\
\hline Lattice Type & hcp & fcc & fct & fcc & fct \\
\hline Hydrogen conc., at. \% & $0-10$ & $0-44$ & $44-50$ & $50-66$ & $66-67$ \\
\hline Lattice Parameter & $\mathrm{a}=2.95$ & $\mathrm{a}=3.305$ & $\mathrm{a}=4.21$ & $\mathrm{a}=4.4$ & $\mathrm{a}=4.5$ \\
& $\mathrm{c}=4.69$ & & $\mathrm{c}=4.6$ & & $\mathrm{c}=4.3$ \\
\hline Atomic Volume & 17.7 & 18 & 20.4 & 21.3 & 21.8 \\
\hline Phase formula & $\mathrm{TiH}_{0.1}$ & $\mathrm{TiH}_{0.3}$ & $\mathrm{TiH}$ & $\mathrm{TiH}_{1.5}$ & $\mathrm{TiH}_{2}$ \\
\hline
\end{tabular}

Another important property of titanium is that it belongs to a class of metal for which the absorption of deuterium is reversible. This means that the dissociation pressure of deuterium gas, which can exist in equilibrium with a metal, is dependent on temperature and on the concentration of the deuterium in the metal. The dissociation pressures of titanium 
deuteride as a function of composition and temperature is known for atomic ratios (deuterium to metal) between 0.02 and 1.8, and for pressure between 3 to $100 m \mu(\mathrm{Hg})$. The determination of dissociation pressure is based on the Clausius-Clapeyron equation for the equilibrium between solid deuteride and gaseous deuterium phases. Graphs of the logarithm of pressure against the reciprocal of temperature give some information on the phases present in the deuteride. These values are given below in Table 1-2[15].

$$
\text { In } P=-(\Delta H / R T)+c
$$

Equation 1

Where $\mathrm{P}=$ equilibrium dissociation pressure at $\mathrm{T}(0 \mathrm{~K})$

$\mathrm{R}=$ the gas constant per mole

$\Delta \mathrm{H}=$ heat absorbed per mole of deuterium

$\mathrm{C}=$ integration constant

Table 1-2: Heat of Dissociation of Titanium Deuteride

\begin{tabular}{|c|c|}
\hline $\begin{array}{l}\text { Atomic ratio } \\
\text { (deuterium to Titanium) }\end{array}$ & $\begin{array}{c}\Delta \boldsymbol{H} \\
\text { Kcal/mole }\end{array}$ \\
\hline 0.020 & 22.53 \\
\hline 0.050 & 22.70 \\
\hline 0.10 & 22.65 \\
\hline 0.20 & 31.77 \\
\hline 0.50 & 31.77 \\
\hline 1.00 & 31.22 \\
\hline 1.20 & 31.44 \\
\hline 1.50 & 30.19 \\
\hline 1.60 & 29.32 \\
\hline 1.80 & 29.14 \\
\hline & \\
\hline
\end{tabular}


Studies conducted to understand the release of hydrogen from titanium due to heat treatment of $\mathrm{TiH}_{2}$ shows that at a temperature lower than $10^{\circ} \mathrm{C} / \mathrm{min}$, the phase transformation sequence is given as $\delta \rightarrow \beta+\delta \rightarrow \beta \rightarrow \alpha+\beta \rightarrow \alpha$. When the heating rate is larger than $10^{\circ} \mathrm{C} / \mathrm{min}$, the transformation is expressed in the form $\delta \rightarrow \beta+\delta \rightarrow \beta$ [16].

\subsubsection{Nuclear Emission}

The mechanism for nuclear emission has been studied in the past and it is hypothesized that neutrons are produced during the warm up phases of the titaniumdeuterium system due to a non-equilibrium conditions such as change in temperature or pressure of the system or both during absorption/desorption of deuterium. However, no nuclear products have been observed in some experiments. The above theory is not complete by itself and does fully explain why neutrons have not been observed in some experiments. Another mechanism called the fracto-fusion mechanism has been proposed to provide an explanation on the means through which nuclear emissions results from condense matter. The fracto-fusion mechanism hypothesizes that nuclear products are as a result of fractures produced in crystal lattice caused by mechanical stress due to hydrogen absorption. The cracks are produced because of internal pressure, or temperature variations, or both in solid matter. The formation of cracks in crystals creates traps that can hold huge amount of deuterium within the crystal structure of solids for deuterium-deuterium interaction to occur leading to a local reaction within the titanium lattice [17-19]. The absorption process occurs much more rapidly in regions where there are cracks than in regions without cracks. It has been proposed that the electric field produced near the crack boundaries is responsible for this effect. Because cracks are formed at different locations, the formation of cracks are certain location in a lattice and not in others can adversely affect the overall nuclear reaction.

Nuclear products such as protons, gammas, neutrons, $\mathrm{x}$-rays, and helium-4 are expected from this process. Fracto-fusion mechanism cannot explain why nuclear products have been 
observed in condense matter systems where cracks were not observed. Nuclear products have also not been observed in systems with large number of cracks.

The detection of nuclear products from condense matter experiments has been subject to a wide number of investigations involving methods such as the gas loading technique, the electrolytic technique, the diffusion technique, the gas discharge method, electro diffusion and the sonic method [20]. The electrolytic method consists of an electrolytic cell with palladium cathode and a platinum cathode subjected to alternative high and low applied currents. The electrolytic cell is placed in an electrolyte. Deuterium is produced from the cathode because of a chemical reaction that occurs on the surface of the electrode and oxygen is produced from the anode. When $D_{2} 0$ is used as an electrolyte, some of the deuterium reacts with the palladium. Examples of electrolytes that have been used include: $\mathrm{LiOD}, \mathrm{Li}_{2} \mathrm{SO}_{4}$, $\mathrm{K}_{2} \mathrm{CO}_{3}$, or $\mathrm{H}_{2} \mathrm{SO}_{4}$. The gas loading method involves introducing hydrogen or deuterium into a suitable material and subjecting it to rapid temperature or pressure change to produce a nuclear effect[20].

Nuclear emissions have been reported in metal hydrides. The detection of these particles is proof that the reaction is indeed nuclear in nature. These reports have been largely inconsistent and there have been issues with reproducible of these results. The nuclear products that have been detected so far include: X-rays, gamma radiation, alpha radiation, beta radiation protons and neutrons.

\subsubsection{Neutron Emission}

Neutrons do not interact directly with matter. Hence detection of neutrons is by indirect means. Neutrons are generally absorbed by low Z material. They can be detected outside of the experimental set-up with relative ease because they will likely penetrate most experimental systems. Most reports suggest that thermal neutron detectors are used in several 
experimental investigations. False signal from noise is an important factor to consider when making such measurements. Neutrons from cosmic rays are another factor that needs to be considered. Boron trifluoride detectors, for example, have been used in the detection of low intensity cosmic neutrons[21]. The method of neutron detection is discussed in chapter III.

Hundreds of experiments have been conducted to investigate neutron emissions. While some of these investigations did not demonstrate any neutron production [22-24], neutrons have been detected in many experiments. The first evidence of neutron production was reported by Jones and coworkers [25]. Jones used an electrolytic cell similar to that utilized by Fleishman and Pons to produced significant neutron flux by passing current in titanium and palladium electrode immersed in an electrolyte containing $\mathrm{D}_{2} \mathrm{O}$ and various salts. Neutrons with $2.45 \mathrm{MeV}$ were detected in their experiment using a liquid organic scintillator (BC-505) placed in a glass cylinder of $12.5 \mathrm{~cm}$ in diameter, in which three glass scintillators plates doped with lithium-6 were placed. Neutron from electrolytic method has also been reported by other groups [27-33]. It was later revealed that it is not necessary to use the electrolysis process to produce neutrons. However, it requires a non-equilibrium condition to achieve this phenomenon. A non-equilibrium condition can be achieved by changing the thermodynamics property of a system. The thermodynamic condition of a system can be change by changing the temperature or pressure or both thereby creating a dynamic condition for the process of absorption and desorption of deuterium in titanium. Several authors have reported neutrons emission from titanium deuterium system by initiating a non-equilibrium condition by repeatedly placing an experimental system in liquid nitrogen followed by rapid warm-up phases[34]. This observation has been confirmed by other authors $[31,32,35,36]$. Table 1-3 shows authors that have reported neutrons emission in their experiments, the method used, type of detector and the neutron count rates. 
Table 1-3: Neutron Detection from LENR

\begin{tabular}{|c|c|c|c|}
\hline Researcher & Method & $\begin{array}{l}\text { Type Neutron } \\
\text { Detector }\end{array}$ & Comment \\
\hline$[25]$ & $\begin{array}{l}\text { Electrolytic } \\
\text { containing Pd or } \mathrm{Ti} \\
\text { immersed in } \\
\text { deuterated water } \\
\end{array}$ & $\begin{array}{l}\text { Liquid organic } \\
\text { scintillator }\end{array}$ & $\sim 2.5 \mathrm{MeV}$ neutrons \\
\hline [34] & $\begin{array}{l}\text { Gas loading of } \\
\text { deuterium in titanium }\end{array}$ & $\begin{array}{l}\mathrm{BF}_{3} \text { neutron } \\
\text { counter }\end{array}$ & $\begin{array}{l}\text { Largest average neutron } \\
\text { emission is about } 1000 \\
\text { counts/h. i.e. } 500 \text { times } \\
\text { above background }\end{array}$ \\
\hline [37] & $\begin{array}{l}\text { Electrolytic using Pd- } \\
\text { Ni electrolytic cell }\end{array}$ & $\begin{array}{l}\mathrm{BF}_{3} \text { neutron } \\
\text { counter } \\
\text { and NE 102A }\end{array}$ & $\begin{array}{l}\text { Large neutron burst of } 2 \times \\
10^{7} \text { neutrons }\end{array}$ \\
\hline$[38]$ & $\begin{array}{l}\text { Gas loading } \\
\text { deuterium into } \mathrm{Ti}\end{array}$ & $\begin{array}{l}\text { plastic } \\
\text { scintillators }\end{array}$ & $\sim 2.5 \mathrm{MeV}$ neutrons \\
\hline [39] & $\begin{array}{l}\text { Mechanical treatment } \\
\text { of titanium in the } \\
\text { presence of heavy } \\
\text { ice, } \mathrm{D}_{2} \mathrm{O} \text {, and LiD }\end{array}$ & $\begin{array}{l}\text { AI-256-6 pulse } \\
\text { height analyzer }\end{array}$ & Weak neutron detected \\
\hline$[40]$ & $\begin{array}{l}\text { Electrolysis using Pd } \\
\text { cathode }\end{array}$ & $\begin{array}{l}\text { NE213 Detector } \\
\text { recoil proton } \\
\text { scintillation } d\end{array}$ & $\sim 2.5 \mathrm{MeV}$ neutrons \\
\hline$[41]$ & $\begin{array}{l}\text { Gas loading apparatus } \\
\text { were of } \mathrm{Ti} / \mathrm{D} \text { system }\end{array}$ & $\begin{array}{l}\text { Plastic scintillators } \\
\text { NEll0 }\end{array}$ & $\sim 2.5 \mathrm{MeV}$ neutrons \\
\hline$[42]$ & $\begin{array}{l}\text { Laser with targets }(\mathrm{Ti}, \\
\mathrm{Si} \text {, and } \mathrm{Pd})\end{array}$ & $\begin{array}{l}\text { spectrometric } \\
\text { neutron detector } \\
\text { D2 consisting of a } \\
\text { counter filled with } \\
\text { a mixture of He3 } \\
\text { and Ar }\end{array}$ & $\begin{array}{l}\text { energies } 2.3-2.6 \mathrm{MeV} \text { were } \\
\text { recorded }\end{array}$ \\
\hline [43] & Pd/D Co-deposition & CR-39 detector & Small neutron flux detected \\
\hline$[44]$ & $\begin{array}{l}\text { Electrolysis using of } \\
\text { Ni-H systems }\end{array}$ & Helium-3 detectors & Small neutron flux detected \\
\hline$[45]$ & Fracture & $\begin{array}{l}\text { Helium-3 } \\
\text { detectors }\end{array}$ & $\begin{array}{l}\text { Small neutron emission } \\
\text { above background }\end{array}$ \\
\hline [36] & $\begin{array}{l}\text { Gas loading titanium } \\
\text { with deuterium }\end{array}$ & Helium-3 detectors & $\begin{array}{l}\text { Over } 2 \text { million neutrons } \\
\text { were counted over a } 5 \text { min } \\
\text { time period }\end{array}$ \\
\hline
\end{tabular}

The conclusion from these results is that the neutron burst observed in this experiment is small and infrequent. It has also been difficult to reproduce some of the results. The reason 
for the irreproducibility is not understood. However, there is overwhelming evidence suggesting neutron emissions in these systems. Therefore, much work still needs to be done to truly understand the behavior of solid hydrides under non-equilibrium conditions. Also, most systems in which low energy neutrons are detected have not be analyzed, making it difficult to understand the true nature of this phenomenon.

\subsubsection{Charged Particles, X-rays and gamma emissions}

Many studies have reported the emissions of charged particles, X-rays and gamma rays that could only result from a nuclear reaction taking place. Some of these emissions were observed after the experiments have been conducted. Charge particles, unlike X-rays and gamma radiations have a short range in matter. Charge particle detectors need to be placed inside or close to the experimental set-up to measure any activity from a cell. CR-39 and surface barrier particle detectors have been used in most studies. Surface barrier detectors are very sensitive to charge particles and generated electric pulses when charge particles pass through the detector. The type of particle detected can be discriminated based on the shape of the height distribution. The CR-39 plastic track detector is usually a $\mathrm{C}_{12} \mathrm{H}_{18} \mathrm{O}_{7}$ polymer with density of about $1.3 \mathrm{~g} / \mathrm{cm}^{3}$. CR-39 has been used in the fields of particle radiation in areas such as aviation, in space, and in the natural environment. It produces tracks within its structure when charge particles go through it. The charged particle tracks become visible and could be investigated using a microscope. Information about the energy and the particle type can be obtained by looking at the track size.

Several authors have reported charge particles tracks from alpha particles[46, 47]. Investigations of charge particle emissions using thin titanium films bombarded with low energy (350-1000 eV) deuterium ions at high current density showed evidence of charge particles emission [48]. Similar evidence of charge particle emissions has been observed by other authors [49-52]. Jones and coworkers [53] found evidence of charge particle emission 
from partially-deuterided titanium foils subjected to non-equilibrium conditions. The experimental investigation suggested that they registered charged particle with counts rate of $2,171 \pm 93$ counts/hour. This count rate was determined to be more than 400 times the background rate. Charge particle was determined to be protons with energy of $2.6 \mathrm{MeV}$ upon exiting the $T i D_{x}$ foil array. Lipson, A., et al. [54] reported evidence of energetic alpha emission (with energy of up to $16.0 \mathrm{MeV}$ ), and proton emission ( energy of $\sim 1.7 \mathrm{MeV}$ ) from a metal surface containing large amount of hydrogen and loaded/excited by electrolysis, glow discharge and a powerful laser. The overwhelming evidence shows that the alpha particles detected has energy between 3-16.5 MeV.

Gamma rays and X-rays are easily detected than charged particles because they have a longer range in matter. Evidence of X-ray emission with energy of $(89 \pm 1) \mathrm{keV}$ from an electrochemical cell made with palladium cathode and platinum anode have been reported. The X-ray films was placed $50 \mathrm{~mm}$ from the experimental system[55, 56]. Karabut et al. [57] reported the observation of $\mathrm{X}$-ray emissions ranging from 0.6 to $10.0 \mathrm{keV}$ in their experiment using high-current glow discharge. The experiments were conducted on the highcurrent glow discharge device using $\mathrm{H}_{2}, \mathrm{D}_{2}, \mathrm{He}, \mathrm{Kr}$, $\mathrm{Ar}$ and $\mathrm{Xe}$ at pressure up to 10 torr, as well as cathode samples made from Al, Sc, V, Ti, Ni, Nb, Zr, Mo, Pd, Ta, W, Pt, at current up to $500 \mathrm{~mA}$ and discharge voltage of $1500-4500 \mathrm{~V}$. X-ray emission with energy of $1.5-2$ $\mathrm{keV}$ and intensity of up to about $100 \mathrm{R} \backslash \mathrm{sec}$ were detected in experiments conducted under high-current glow discharge in deuterium and hydrogen using Pd cathodes[58]. Palladium exposed to pressurized deuterium gas at 60 atm and $198 \mathrm{~K}$ and the temperature cycled to up to $593 \mathrm{~K}$, beyond the critical point for palladium deuteride, showed evidence of excess neutrons and gamma rays above background levels. A similar investigation, however, with an empty cell or and with hydrogen-palladium cell did not show any sign of excess neutrons or gamma rays over the background levels[59]. 


\subsubsection{Helium-4 and Tritium Production}

Tritium and ${ }^{4} \mathrm{He}$ are two products expected from d-d fusion reaction. Considerable work has been done to detect the presence of these two products in PdD and TiD systems. Neutron with energy of $14 \mathrm{MeV}$ is expected to be produced with tritium with a neutron/tritium ratio of about $1 \times 10^{-4}$. So far, $14 \mathrm{MeV}$ neutrons has never been detected alongside tritium. Different methods have been explored to identify tritium. Tritium can be detected by performing an elemental analysis of the gaseous content from a PdD reaction with a mass spectrometer or by using a nuclear radiation detection system such as the liquid scintillation detector to detect the $18 \mathrm{KeV}$ beta emitted from the radiative decay of tritium. Experiments of this nature generally requires care and some level of sophistication because tritium from the environmental can contaminate the experimental systems therefore producing false signals. There is also the possibility that control samples contained some levels of tritium. It is therefore necessary to differentiate the tritium that is detected in these systems.

Tritium has a half-life of about 12.3 years and emits a weak beta radiation with a maximum $18.6 \mathrm{keV}$ and an average energy of $5.7 \mathrm{keV}$. Windowless detectors are mostly used to detect tritium because the weak beta radiation hardly penetrates the window of most detectors. The presence of tritium in $T i D_{x}$ can be determined by directly counting the beta radiation from the decay of tritium. An extensive investigation was conducted by a group at Trombay to detect the tritium emission from their experimental system using methods such as variation of the gas loading procedures and induction heating of single machined titanium targets in a glass chamber, and a plasma focus device for deuteriding its central titanium electrode. The presence of tritium was detected by direct measurement of the beta radiation emitted from the decay of tritium. The experimental samples containing titanium were analyzed using an autoradiograph. The autoradiograph is a technique that can produce 
radiation emission zones from which a spatial distribution of tritium in titanium samples can be establish. The experimental samples were exposed to a medical X-ray film for several hours. Their investigation revealed the presence of large concentration of tritium in highly localized spots with each spot containing about $10^{12}$ to $10^{14}$ ( 2 to $200 \mathrm{~Bq}$ ) atoms of tritium [60]. The large amount of tritium observed in this experiment makes it difficult to dismiss it. Worth noting is the fact that many samples were loaded with deuterium gas but only a few samples showed the presence of tritium. Samples with large loading ratio did not show the presence of tritium. It is difficult to explain why tritium was not found in samples with large loading ratio. It has been hypothesized that the large concentration of tritium at these localized spots is because of a cascading reaction or micronuclear explosions occurring at specific sites in the titanium lattice. The presence of tritium has been found in some aged deuterium targets that were used by the BARC group from 1972 to 1986 using the autoradiography technique [61]. It was reported that the content of tritium in this samples were about $0.3-150 \mathrm{MBq}$ or had a tritium/deuterium ratio ranging from 0.007 to $3.5 \times 10^{-4}$. However, no localized spots were found in these samples. The large tritium observed in this sample may be because the nuclear reaction has been taken place throughout the years.

The presence of tritium has also been observed in a wide variety of systems: palladium as cathode in $\mathrm{D}_{2} \mathrm{O}$ - based electrolyte, $\mathrm{Ni}$ as a cathode in $\mathrm{H}_{2} \mathrm{O}$ - based electrolytes, low-voltage discharge of Pd electrodes on low pressure deuterium, gas loading of titanium with high pressure deuterium gas followed by rapid temperature change, low voltage discharge of palladium electrodes loaded with deuterium at high pressures which is rapid release from palladium after heating[62]. A summary of the results showing tritium measurements from 1990 to 1993 is shown in Table 1-4 which can be found in the book by Storm[20]. The methods used in the experimental investigation has been represented by letters. The first letter is the environment in which tritium was observed. The letter that follows shows the method that was 
used to determine the presence of tritium as shown in Table 1-6 and the symbol that follows shows the type of cell that was used in the investigation, where "CS" means close sealed cells, other methods involved measuring tritium leaving or entering the cell denoted here by "L"[62].

Table 1-4: Tritium Measurements in Different Systems

\begin{tabular}{|c|c|c|c|c|}
\hline Researcher & Method & $\begin{array}{l}\text { Total Tritium } \\
\text { atoms }\end{array}$ & $\begin{array}{l}\text { Average } \\
\text { Rate } \\
\text { atoms/sec }\end{array}$ & Remark \\
\hline$[63]$ & $\mathrm{I}, 1, \mathrm{~L}$ & $10^{15}$ & $1 \times 10^{8}$ & $\begin{array}{l}\text { Tritium production } \\
\text { could be turn on and off }\end{array}$ \\
\hline [64] & $\mathrm{I}, 1, \mathrm{~L}$ & $10^{10}$ & & $\begin{array}{l}\text { Several assumptions } \\
\text { makes result uncertain }\end{array}$ \\
\hline \multirow[t]{3}{*}[65]{} & IV,2,CS & & $3 \times 10^{11}$ & $\begin{array}{l}\text { Use a pulse current } \\
\text { discharge between } \\
\text { oxidized Pd and Si in } \\
\text { pressurized deuterium. } \\
\text { Many cells produced } \\
\text { tritium but not when } \\
\text { hydrogen was used. }\end{array}$ \\
\hline & IV,2,CS & $10^{12}$ & $10^{7}$ & $\begin{array}{l}\text { Wires of palladium after } \\
\text { purification were loaded } \\
\text { with deuterium and } \\
\text { voltage was applied. }\end{array}$ \\
\hline & $\mathrm{IV}, 2, \mathrm{CS}$ & $10^{12}$ & $10^{6}$ & $\begin{array}{l}\text { Pulse glow discharge } \\
\text { between Pd plate and } \\
\mathrm{Pd} \text { wire. Pd was } \\
\text { reanalyzed for tritium. }\end{array}$ \\
\hline [66] & $\mathrm{IV}, 2, \mathrm{CS}$ & $\approx 10^{12}$ & & $\begin{array}{l}\text { Titanium alloy partially } \\
\text { reacted with deuterium } \\
\text { and subjected to } \\
\text { temperature change, } \\
\text { Neutrons detected as a } \\
\text { result. }\end{array}$ \\
\hline [67] & $\mathrm{I}, 1, \mathrm{~L}$ & $8 \times 10^{15}$ & & $\begin{array}{l}\text { Pd-Ag electrolyzed } \\
\text { in } \mathrm{NaOD}^{-} \mathrm{D}_{2} \mathrm{O} \text {. Many } \\
\text { electrolytes show } \\
\text { successful results }\end{array}$ \\
\hline$[68]$ & II, 1 & $10^{10}$ & $4 \times 10^{5}$ & 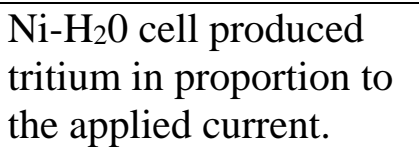 \\
\hline [69] & II,I & & $10^{14}$ & $\begin{array}{l}14 \mathrm{Ni}^{-} \mathrm{H}_{2} \mathrm{O} \text { cells } \\
\text { produced tritium in } \\
\text { proportion to the } \\
\text { applied current. }\end{array}$ \\
\hline [70] & $\mathrm{IV}, 2,5, \mathrm{CS}$ & $\sim 10^{16}$ & & $\begin{array}{l}\text { TiD chips cooled in } \\
\text { liquid }\end{array}$ \\
\hline
\end{tabular}




\begin{tabular}{|l|l|l|l|l|}
\hline$[71]$ & I,1,L & $\sim 10^{16}$ & & $\begin{array}{l}\text { Cells enclosed and total } \\
\text { inventory made. Many } \\
\text { cells studied but only a } \\
\text { few produced tritium }\end{array}$ \\
\hline$[72]$ & I,L & $10^{11}$ & $10^{5}$ & $\begin{array}{l}\text { The 4 successful cells } \\
\text { were sealed. No tritium } \\
\text { seen when } \mathrm{H}_{2} \mathrm{O} \text {-based is } \\
\text { used. }\end{array}$ \\
\hline$[73]$ & I,1,CS & $10^{11}$ & $10^{5}$ & $\begin{array}{l}\text { The 4 successful cells } \\
\text { were sealed. No tritium } \\
\text { found when } \mathrm{H}_{2} \mathrm{O} \text {-based } \\
\text { cells were used. }\end{array}$ \\
\hline
\end{tabular}

\section{Table 1-5: Detection Environment used in Table 1-5}

1. Palladium used as cathode in $\mathrm{D}_{2} \mathrm{O}$ - based electrolyte,

2. Ni used as a cathode in $\mathrm{H}_{2} \mathrm{O}$ - based electrolytes,

3. Low-voltage discharge involving Pd electrodes on low pressure $D_{2}$,

4. low voltage discharge involving Pd electrodes in high pressure $D_{2}$

5. Rapid release from Pd upon heating

6. Gas loading of titanium with high pressure $D_{2}$ followed by temperature change,

\section{Table 1-6: Method used to detect Tritium}

1. Scintillation material which emits light as beta radiation deposits its energy or passes through it. This method is useful in measuring the energy of the beta radiation. The detection limit of this system is about $10^{7}$ atoms.

2. Ionization chamber in which the current generated by the $\beta$-particles is amplified and subsequently measured. This method is used when tritium is in the gas phase and has a detection limit of nearly $10^{9}$ atoms.

3. Mass spectrometer.

4. This method of analyses is based in determining the amount of tritium based in how much has decayed upon entering the material. The result is weighted mean of the tritium present in the past. The detection limits are about $10^{4}$ atoms.

5. The autograph detects beta particles or x-ray radiation when the film is exposed to this particle. Detail map of the particle tracks can be established. The detection limit of this method is large and can be as high as $10^{12-13}$ atoms.

These findings indicate that the ratio of tritium to neutron production varies from $10^{4}$ to $10^{9}$.

Helium-4 is another product emitted from nuclear reaction involving deuterium. When ${ }^{4} \mathrm{He}$ is released, a gamma ray is expected as well. Previous claims about ${ }^{4} \mathrm{He}$ production were rejected because gamma radiation was not detected. There are several ways of making ${ }^{4} \mathrm{He}$ without using fusion. Table 1-7 shows the different ways of producing neutrons. 
Table 1-7: Proposed Reaction from ${ }^{4} \mathrm{He}$ production[74]

\begin{tabular}{|c|c|c|}
\hline Reaction & MeV/He & He atoms/J \\
\hline $\mathrm{D}+\mathrm{D} \rightarrow{ }_{2}^{4} \mathrm{He}+$ energy & 23.8 & $2.6 \times 10^{11}$ \\
\hline $\mathrm{D} \mathrm{D} \rightarrow{ }_{2}^{4} \mathrm{Be} \rightarrow 2{ }_{2}^{4} \mathrm{He}$ & 23.8 & $2.6 \times 10^{11}$ \\
\hline $\mathrm{D}+\mathrm{T} \rightarrow{ }_{2}^{4} \mathrm{He}+$ neutrons & 17.5 & $3.6 \times 10^{11}$ \\
\hline $\mathrm{D}+{ }_{3}^{6} \mathrm{Li} \rightarrow{ }_{4}^{8} \mathrm{Be} \rightarrow 2{ }_{2}^{4} \mathrm{He}$ & 11.2 & $5.6 \times 10^{11}$ \\
\hline $\mathrm{H}+{ }_{3}^{7} \mathrm{Li} \rightarrow{ }_{4}^{8} \mathrm{Be} \rightarrow 2{ }_{2}^{4} \mathrm{He}$ & 8.4 & $7.4 \times 10^{11}$ \\
\hline $2 \mathrm{H}+{ }_{3}^{7} \mathrm{Li} \rightarrow{ }_{4}^{9} \mathrm{Be} \rightarrow 2{ }_{2}^{4} \mathrm{He}+\mathrm{p}$ & 8.4 & $4.7 \times 10^{11}$ \\
\hline $\mathrm{n}+{ }_{3}^{7} \mathrm{Li} \rightarrow{ }_{4}^{8} \mathrm{Be}+\operatorname{beta}(13 \mathrm{MeV}) \rightarrow 2{ }_{2}^{4} \mathrm{He}$ & 13.4 & $14.5 \times 10^{11}$ \\
\hline $\mathrm{n}+{ }_{3}^{6} \mathrm{Li} \rightarrow{ }_{2}^{4} \mathrm{He}+\mathrm{T}$ & & \\
\hline
\end{tabular}

The quadruple mass spectrometer can be used to detect the presence of ${ }^{4} \mathrm{He}$ using the gases from the experimental systems. The Quadruple mass spectrometer is widely used to determine the compositions, to measure isotopic ratios, and for detecting leaks in vacuum systems. In the mass spectrometer, an electric field is used to accelerate ions out of the source into the quadrupole analyzer. The quadrupole analyzers consist of four electrodes or rods place parallel to each other. The quadrupole is used to filter ions according to their mass to charge $(\mathrm{m} / \mathrm{e})$ ratio as they go through the analyzer. Combined applied voltage (DC) and radio frequency (RF) potentials on the quadrupole rods can be adjusted such that only particles with selected mass-to-charge ratio are allowed to go through. The advantage of the quadrupole analyzer is that the instrument is relatively small, low-cost systems, has fast scan rate, and high transmission efficiency. However, the analyzer has a limited resolution. The resolution is limited to the $\mathrm{m} / \mathrm{z}$ ratio. Many of these units have a mass range of $\mathrm{m} / \mathrm{e}$ of 1000 . The detection of ${ }^{4} \mathrm{He}$ requires considerable care to prevent the contamination of ${ }^{4} \mathrm{He}$ from the environment. Even after measurements, considerable analysis may be needed to evaluate 
these results. Helium-4 has been observed during the electrolysis of $D_{2} O$ in palladium electrode [75], and in palladium containing 0.1 M LIOD with counts rate of about $10^{3}$ times above background levels[76]. Botta et al. [77] in their search for ${ }^{4} \mathrm{He}$ from palladium sheets loaded with deuterium gas reported the production of ${ }^{4} \mathrm{He}$ from their experimental system. The ${ }^{4} \mathrm{He}$ analysis was performed after a 500-hour run. The high-resolution Q-mass spectrometry (ULVAC HI-RESOM 2SM) was used in this measurement. They concluded that the percentage of ${ }^{4} \mathrm{He}$ produced increased by $20-30 \%$ while the residual pressure was lowered by $\sim 15 \%$. However, they also detected ${ }^{4} \mathrm{He}$ from their background analysis. The ${ }^{4} \mathrm{He}$ was detected from the background is troubling!

\subsubsection{Theories and Mechanism on Neutron Production}

Several theories have been proposed to explain neutron production. These theories are generally based on assumptions about the titanium-deuterium system. So far, no theory has been able to provide an effective explanation on the mechanism of nuclear emissions. Most theories are not widely accepted. As a result, a limited discussion about the proposed theories will be given in this section. We will explore some of the theories that are important for neutron production.

\subsection{5 "Phase Transition Mechanism"}

The mechanism takes advantage of large pressure build-up in the crystal structure in transition metals created by phase changes during thermal shocking of titanium or palladium loaded with deuterium from liquid nitrogen $\left(-196^{\circ} \mathrm{C}\right)$ to about $100^{\circ} \mathrm{C}[36]$. The thermal shock causes an abrupt pressure change in the titanium lattice where deuterium atoms are trapped in defects on a time scale much faster than diffusion times. The local pressure increase will cause the temperature to increase and a local fusion reaction to occur. Neutron emission results as a consequence of this process. This method is similar to inertial confinement fusion except that the volume would be constant, and pressure would be varied. 


\subsubsection{Fracto-fusion Mechanism}

The fracto-fusion theory has been proposed to explain the means through which nuclear emissions results from condense matter. According to the fracto-fusion mechanism, neutrons are produced as a result of fracture caused by mechanical stress in crystals lattice. Cracks could result from internal pressure, or temperature variations, or both in solid matter. The formation of cracks in crystals creates traps that can hold huge amount of deuterium within the crystal structure of solids for titanium-deuterium interaction to occur leading to a local nuclear reaction [1-3]. It has been proposed that the electric field produced near the crack boundaries is responsible for this effect. Nuclear products such as protons, gammas, neutrons, $\mathrm{x}$-rays, and helium-4 are expected from this process.

\subsubsection{Widom-Larsen Theory}

Widom and Larsen[78, 79] theory is based on ultra-low momentum neutron catalyzed nuclear reactions on metallic hydride surfaces. Widom-Larsen theory predicts that under special conditions, ultra-low momentum neutrons are produced in condensed matter environment via weak interactions involving the capture of "heavy" electrons by protons. The Coulomb barrier does not prevent the neutron catalyzed nuclear reactions from occurring. The neutrons are produced with an ultra-low momentum because of the size of the coherence domain of the oscillating protons. The ultracold neutrons have very large nuclear absorption cross sections and are therefore efficiently absorbed by the surrounding nuclei. Some of the neutrons may escape the vicinity and are hardly detected. The ultra-low momentum neutrons may produce "neutron rich" nuclei in substantial quantities or initiate low energy nuclear reaction through further nuclear reaction. The production of ultra-low momentum neutrons can induce chains of nuclear reactions in neighboring condensed matter. 


\subsubsection{Multibody fusion Model}

The multibody fusion model proposed by Takahashi et al. [80, 81] suggests that neutrons may be as a results of a multibody fusion of hydrogen isotopes in metal lattice. A transient dynamic in metal deuterides can generate close pairs and clusters of deuterons with time-dependent deep atomic potential thereby inducing a strong screening effect on the Coulomb barrier penetration. It has found using numerical estimations of reaction rates for 2D, 3D, and 4D fusion processes in $\mathrm{PdDx}$ and TiDx that the major generation of ${ }^{4} \mathrm{He}$ is by $\mathrm{H}+2 \mathrm{D}, 3 \mathrm{D}, \mathrm{H}+3 \mathrm{D}$, and 4D. This reaction results in neutron production[40]. However, the multibody fusion model cannot by itself explain the neutron emissions in condense matter systems [82].

\subsubsection{Trapped Neutron Catalyzed Model for Cold Fusion (TNCF Model)}

The theory proposed a possibility of the occurrence of the fusion leading to the release of so many neutrons, anomalous excess heat and tritium from the d-d fusion reaction triggered by trapped thermal neutrons in materials[83]. The rare observation of $6.25 \mathrm{MeV}$ and 2.22 MeV photons proposed by the TNCF Model leaves questions about the model. There is also not enough evidence to support the existence of trapped neutrons in materials. 


\section{REFERENCES}

1. Dearnaley, G. and R.E. Watkins, Titanium and its alloys. 1984, Google Patents.

2. Leyens, C. and M. Peters, Titanium and titanium alloys. 2003: Wiley Online Library.

3. Peters, M., et al., Structure and properties of titanium and titanium alloys. Titanium and Titanium Alloys: Fundamentals and Applications, 2003: p. 1-36.

4. Ionete, E.I., B. Monea, and M. Zamfirache. Hydrogen and deuterium sorption on titanium under vacuum conditions. in Proceedings of the 2nd international conference on Circuits, systems, control, signals. 2011. World Scientific and Engineering Academy and Society (WSEAS).

5. $\quad$ Olayo, M., et al., Sorption of hydrogen in titanium plates at low pressure. International journal of hydrogen energy, 1998. 23(1): p. 15-18.

6. Kasemo, B., E. Törnqvist, and P. Johansson, Anomalous desorption of H2 from TiHx films, $0.3 \lesssim x \lesssim 1.8$. Chemical Physics Letters, 1979. 68(2-3): p. 416-419.

7. Tal-Gutelmacher, E. and D. Eliezer, The hydrogen embrittlement of titanium-based alloys. Jom, 2005. 57(9): p. 46-49.

8. Matysina, Z. and D. Shchur, Phase Transformations $\alpha \rightarrow \beta \rightarrow \gamma \rightarrow \delta \rightarrow \varepsilon$ in Titanium Hydride TiHx with Increase in Hydrogen Concentration. Russian physics journal, 2001. 44(11): p. 1237-1243.

9. San-Martin, A. and F. Manchester, The H-Ti (hydrogen-titanium) system. Journal of Phase Equilibria, 1987. 8(1): p. 30-42.

10. Matysina, Z. and D. Shchur, Phase Transformations $\alpha \rightarrow \beta \rightarrow \gamma \rightarrow \delta \rightarrow \varepsilon$ in Titanium Hydride TiH $x$ with Increase in Hydrogen Concentration. Russian physics journal, 2001. 44(11): p. 1237-1243.

11. Schur, D., et al., Phase transformations in titanium hydrides. International journal of hydrogen energy, 1996. 21(11): p. 1121-1124.

12. Wedler, G. and H. Strothenk, Elektrische und kalorimetrische Messungen am System Titan/Wasserstoff bei 273 K. Zeitschrift für Physikalische Chemie, 1966. 48(1_2): p. 86-101.

13. McQuillan, A.D. An experimental and thermodynamic investigation of the hydrogentitanium system. in Proceedings of the Royal Society of London A: Mathematical, Physical and Engineering Sciences. 1950. The Royal Society.

14. Stalinski, B., C. Coogan, and H. Gutowsky, Proton Magnetic Resonance Studies of Structure, Diffusion, and Resonance Shifts in Titanium Hydride. The Journal of Chemical Physics, 1961. 34(4): p. 1191-1206.

15. Morton, J. and D. Stark, The dissociation pressures of titanium and zirconium deuterides as functions of composition and temperature. Transactions of the Faraday Society, 1960. 56: p. 351-356.

16. Ma, M., et al., Phase transformations of titanium hydride in thermal desorption process with different heating rates. International Journal of Hydrogen Energy, 2015. 40(29): p. 8926-8934.

17. Takeda, T. and T. Takizuka, Fractofusion mechanism. Journal of the Physical Society of Japan, 1989. 58(9): p. 3073-3076.

18. Segre, S., et al., A mechanism for neutron emission from deuterium trapped in metals. EPL (Europhysics Letters), 1990. 11(3): p. 201.

19. Yasui, K., Fractofusion mechanism. Fusion Science and Technology, 1992. 22(3): p. 400-406.

20. Storms, E., A Student's Guide to Cold Fusion. Library LERN-CANR. Org, 2003. 
21. Chen, C.-Y. and C. Chung, Low intensity cosmic neutron measurements using a portable BF3 counting system. Nuclear Instruments and Methods in Physics Research Section A: Accelerators, Spectrometers, Detectors and Associated Equipment, 1997. 395(2): p. 195-201.

22. Arata, Y. and Y.-C. Zhang, Achievement of an intense cold fusion reaction. Fusion Science and Technology, 1990. 18(1): p. 95-102.

23. Fralick, G.C., A.J. Decker, and J.W. Blue, Results of an Attempt to Measure Increased Rates of the Reaction $2 \mathrm{D}+2 \mathrm{D} \rightarrow 3 \mathrm{He}+n$ in a Non-electrochemical Cold Fusion Experiment. NASA Technical Memorandum, 1989. 102430: p. 1-15.

24. Ziegler, J., et al., Electrochemical experiments in cold nuclear fusion. Physical review letters, 1989. 62(25): p. 2929.

25. Jones, S.E., et al., Observation of cold nuclear fusion in condensed matter. Nature, 1989. 338(6218): p. 737-740.

26. Gozzi, D., et al., Neutron and tritium evidence in the electrolytic reduction of deuterium on palladium electrodes. Fusion Science and Technology, 1992. 21(1): p. 60-74.

27. Mizuno, T., et al., Neutron evolution from a palladium electrode by alternate absorption treatment of deuterium and hydrogen. Japanese Journal of Applied Physics, 2001. 40(9A): p. L989.

28. Pitt, W.G., J.N. Harb, and C.J. Farahmandi. Observation of Neutrons During Electrolysis of LiOD Solutions. in Proceedings: EPRI-NSF Workshop on Anomalous Effects in Deuterided Metals. 1989. Citeseer.

29. Izumida, T., et al., A search for neutron emission from cold nuclear fusion in a titanium-deuterium system. Fusion Science and Technology, 1990. 18(4): p. 641-646.

30. Sato, T., et al., Detection of neutrons in electrolysis of heavy water. Fusion Science and Technology, 1991. 19(2): p. 357-363.

31. Zhu, R., et al., Measurement of Neutron Burst Production in Thermal Cycle of D 2 Absorbed Titanium Chips. Fusion Science and Technology, 1991. 20(3): p. 349-353.

32. Chicea, D. and D. Lupu, Low-Intensity Neutron Emission from TiD x Samples Under Nonequilibrium Conditions. Fusion Science and Technology, 2001. 39(1): p. 108-113.

33. Takahashi, A., et al. Neutron spectra anc controllability by PdD/Electrolysis cell with low-high current pulse operation. in Proc. ICCf2. 1991.

34. De Ninno, A., et al., Evidence of emission of neutrons from a titanium-deuterium system. EPL (Europhysics Letters), 1989. 9(3): p. 221.

35. Menlove, H., et al. Low-background measurements of neutron emission from Ti metal in pressurized deuterium gas. in Proc. ICCF2. 1991.

36. Prelas, M.A. and E. Lukosi. Neutron emission from cryogenically cooled metals under thermal shock. in Proceedings 17 th International Conference on Cold Fusion, Daejeon/Korea. 2012.

37. Iyengar, P.K. Cold fusion results in BARC experiments. 1989.

38. Bressani, T., et al., Observation of $2.5 \mathrm{MeV}$ neutrons emitted from a titaniumdeuterium system. Il Nuovo Cimento A (1965-1970), 1991. 104(9): p. 1413-1416.

39. Lipson, A.G., et al., Neutron emission during the mechanical treatment of titanium in the presence of deuterated substances. ZhETF Pisma Redaktsiiu, 1989. 49: p. 588.

40. Takahashi, A., et al., Emission of $2.45 \mathrm{MeV}$ and higher energy neutrons from D2O-Pd cell under biased-pulse electrolysis. Journal of Nuclear Science and Technology, 1990. 27(7): p. 663-666.

41. Botta, E., et al., Measurement of $2.5 \mathrm{MeV}$ neutron emission from Ti/D and $P d / D$ systems. Il Nuovo Cimento A (1965-1970), 1992. 105(11): p. 1663-1671. 
42. Volkov, R.V., et al., Neutron generation in dense femtosecond laser plasma of a structured solid target. Journal of Experimental and Theoretical Physics Letters, 2000. 72(8): p. 401-404.

43. Szpak, S., et al. LENR research using co-deposition. in Proc. the 14th Int. Conf. on Condensed Matter Nuclear Science, Washington, DC. 2008.

44. Battaglia, A., et al., Neutron emission in Ni-H systems. Il Nuovo Cimento A, 1999. 112(9): p. 921-931.

45. Carpinteri, A., F. Cardone, and G. Lacidogna, Piezonuclear neutrons from brittle fracture: early results of mechanical compression tests. Strain, 2009. 45(4): p. 332339.

46. Jin, S.-X., F. Zhang, and Y. Liu, Deuterium Absorbability and Anomalous Nuclear Effect of YBCO High Temperature Super-Conductor. Fusion Technology, 1994. 26(4): p. 527-529.

47. Dong, S., et al., Precursors to "Cold Fusion" Phenomenon and the Detection of Energetic Charged Particles in Deuterium/Solid Systems. Fusion Science and Technology, 1991. 20(3): p. 330-333.

48. Chambers, G., G. Hubler, and K. Grabowski. Search for energetic charged particle reaction products during deuterium charging of metal lattices. in Anomalous nuclear effects in deuterium/solid systems. 1991. AIP Publishing.

49. Iida, T., et al., Deuteron Fusion Experiment with Ti and Pd Foils Implanted with Deuteron Beams II. Product ID: TR-104188-V3 Sector Name: Nuclear Date Published: 7/28/1994 Document Type: Technical Report File size: 18.87 MB File Type: Adobe PDF (. pdf) Full list price: No Charge, 1994. 1993.

50. Dawei, M., Z. Li, and C. Boxian, Real time measurements of the energetic charged particles and the loading ratio (D/Pd), in Frontiers of cold fusion. 1993.

51. Taniguchi, R., Characteristic peak structures on charged particle spectra during electrolysis experiment. Fusion Technology, 1994. 26(4): p. 186-191.

52. Yamaguchi, E. and T. Nishioka. Direct evidence for nuclear fusion reactions in deuterated palladium. in Frontiers of Cold Fusion, Proceedings of the Third International Conference on Cold Fusion. 1993. Citeseer.

53. Jones, S., et al. Charged-particle emissions from metal deuterides. in Proceedings of the Tenth International Conference on Cold Fusion. 2003.

54. Lipson, A., et al. Phenomenon of an energetic charged particle emission from hydrogen/deuterium loaded metals. in Tenth International Conference on Cold Fusion. 2003.

55. Cellucci, F., et al. $x$-ray, heat excess and 4 He in the electrochemical confinement of deuterium in palladium. in Sixth International Conference on Cold Fusion, Progress in New Hydrogen Energy. 1996.

56. Urciuoli, G., Erratum to ' $X$-ray, heat excess and 4 He in the D/Pd system' [J. Electroanal. Chem. 435 (1997) 113]. Journal of Electroanalytical Chemistry, 1998. 452: p. 251-271.

57. Karabut, A. and E. Karabut. Research into spectra of X-ray emission from solid cathode medium during and after high current glow discharge operation. in 14th International Conference on Condensed Matter Nuclear Science. 2008. Citeseer.

58. Karabut, A., X-ray emission in the high-current glow discharge experiments. Condensed Matter Nuclear Science, Proc. ICCF9, edited by Xing Z. Li, 2002: p. 155.

59. Jorne, J., Neutron and gamma-ray emission from palladium deuteride under supercritical conditions. Fusion Science and Technology, 1991. 19(2): p. 371-374.

60. Srinivasan, M., et al., Observation of tritium in gas/plasma loaded titanium samples. AIP Conference Proceedings, 1991. 228(1): p. 514-534. 
61. Srinivasan, M., et al., Observation of High Tritium Levels in Aged Deuterated Titanium Targets as Possible Evidence of Cold Fusion. Fusion Technol, 1990. 18: p. 88.

62. Storms, E., A Review of the Cold Fusion Effect. J. Sci. Exploration, 1996. 10(2): p. 185.

63. Bockris, J.O.M., et al., Tritium and helium production in palladium electrodes and the fugacity of deuterium therein. Proc. ICCF3 (October 21-25, 1992, Nagoya, Japan), 1993: p. 231.

64. Chene, J. and A. Brass, Tritium production during the cathodic discharge of deuterium on palladium. Journal of electroanalytical chemistry and interfacial electrochemistry, 1990. 280(1): p. 199-205.

65. Claytor, T., et al., Tritium and neutron measurements from deuterated Pd-Si. 1990, Los Alamos National Lab., NM (USA).

66. D'Amato, F., et al. Search for Nuclear Phenomena by the Interaction between Titanium and Deuterium. in Proceedings of the First Annual Conference on Cold Fusion. 1990.

67. Iyengar, P., et al., Bhabha Atomic Research Centre studies in cold fusion. Fusion Science and Technology, 1990. 18(1): p. 32-94.

68. Notoya, R., Alkali-hydrogen cold fusion accompanied by tritium production on nickel. Trans Fusion, 1994.

69. Ramamurthy, H., et al. Further studies on excess heat generation in $\mathrm{Ni}-\mathrm{H} 2 \mathrm{O}$ electrolytic cells. in Proc. 4th Int. Conf. Cold Fusion. 1993.

70. Srinivasan, M., et al. Observation of tritium in gas/plasma loaded titanium samples. in AIP Conference Proceedings. 1990.

71. Storms, E. and C. Talcott, Electrolytic tritium production. Fusion Science and Technology, 1990. 17(4): p. 680-695.

72. Szpak, S., P. Mosier-Boss, and J. Smith. Reliable procedure for the initiation of the Fleischmann-Pons Effect. in Second Annual Conference on Cold Fusion," The Science of Cold Fusion. 1991.

73. Will, F.G., K. Cedzynska, and D.C. Linton. Tritium generation in palladium cathodes with high deuterium loading. in Fourth International Conference on Cold Fusion. 1993.

74. Storms, E., Status of cold fusion (2010). Naturwissenschaften, 2010. 97(10): p. 861881.

75. Bush, B.F., et al., Helium production during the electrolysis of D2O in cold fusion experiments. Journal of Electroanalytical Chemistry and Interfacial Electrochemistry, 1991. 304(1): p. 271-278.

76. Chien, C.-C., et al., On an electrode producing massive quantities of tritium and helium. Journal of Electroanalytical Chemistry, 1992. 338(1): p. 189-212.

77. Botta, E., et al. Search for 4 He production from Pd/D2 systems in gas phase. in 5th International Conference on Cold Fusion. 1995.

78. Widom, A. and L. Larsen, Ultra low momentum neutron catalyzed nuclear reactions on metallic hydride surfaces. The European Physical Journal C-Particles and Fields, 2006. 46(1): p. 107-111.

79. Srivastava, Y., A. Widom, and L. Larsen, A primer for electroweak induced lowenergy nuclear reactions. Pramana, 2010. 75(4): p. 617-637.

80. Takahashi, A., et al., Excess heat and nuclear products by D2O/Pd electrolysis and multibody fusion. Int. J. Appl. Electromagn. Mater, 1992. 3: p. 221.

81. Takahashi, A., et al., Multibody fusion model to explain experimental results. Fusion Science and Technology, 1995. 27(1): p. 71-85. 
82. Fleischmann, M., S. Pons, and G. Preparata, Possible theories of cold fusion. Il Nuovo Cimento A (1965-1970), 2008. 107(1): p. 143.

83. Kozima, H. and S. Watanabe. Nuclear processes in trapped neutron catalyzed model for cold fusion. in 5th International Conference on Cold Fusion. 1995. 


\section{NEUTRON DETECTION}

Our discussion in this section will be limited to slow and fast neutron detection. Neutrons with energy below the cadmium cut-off value of about $0.5 \mathrm{eV}$ are referred to as slow neutrons and are distinguished from intermediate and fast neutrons that have energies above this value. Thermal neutrons refer to neutrons that have been slowed down to equilibrium with their surrounding medium. Slow neutron reactions are exothermic reactions, while fast neutron reactions are endothermic reaction. The detection of neutrons is by indirect means since neutrons are not charged particles. Neutron detection relies on conversion processes that leads to the release of secondary charged particles such as protons, alpha particles, etc. when neutrons interact with matter. These charged particles cause excitation or ionizing of atoms along its path until all its energy is completely absorbed. The charge generated from secondary particles can be collected and processed by a detector system to obtain useful information.

Neutron detectors consist of a target or a combination of target material used to produce the conversion process which results in neutron detection. The choice of material used in neutron detection depends on the neutron cross section. Neutron cross section has roughly a $1 / \sqrt{E}$ dependence. As a result, different techniques need to be employed to detect neutrons of different energies. Neutron interaction with matter could result in absorption or scattering reaction. Scattering reactions lead to a change in energy or direction of motion of the neutron but does not result in disappearance of the neutron. In absorptive reaction, the neutron is completely absorbed, and a compound nucleus is formed. A formation of a compound nucleus occurs when the sum of the binding and kinetic energies of the incident neutron is equivalent to the excited state of the compound nucleus. A variety of nuclear 
emission(s) such as alphas, protons or gamma photons may follow. Slow neutron detection is based on this type of reaction[1].

The probability of a nuclear reaction occurring depends on the energy of the neutron and the property of the target nuclei. In choosing a nuclear reaction for neutron detection, the cross section for the reaction must be as large as possible so that high efficiency detectors can be constructed with a relatively small dimension. It is also important that the material be available naturally with high atomic abundance or that one should be able to manufacture such material artificially with relative ease. The cross sections of some materials significant for neutron detection are shown in Figure 2-1. Helium-3, lithium-6 and boron-10 are commonly used material for slow neutron detection because of their large thermal neutron cross sections. ${ }^{3} \mathrm{He}$ has the largest thermal neutron cross section for thermal neutrons among these three materials while hydrogen has largest cross section for fast neutrons. The range of charged particles in these materials is very important, especially if they are to be used in the gaseous form in gas-filled detectors because the distance travelled by the reaction product plays a valuable role in the detector design. This requirement is easily met in solid state neutron detectors where the range of charged particles does not exceed a few tenths of a millimeter in solid material[2]. 


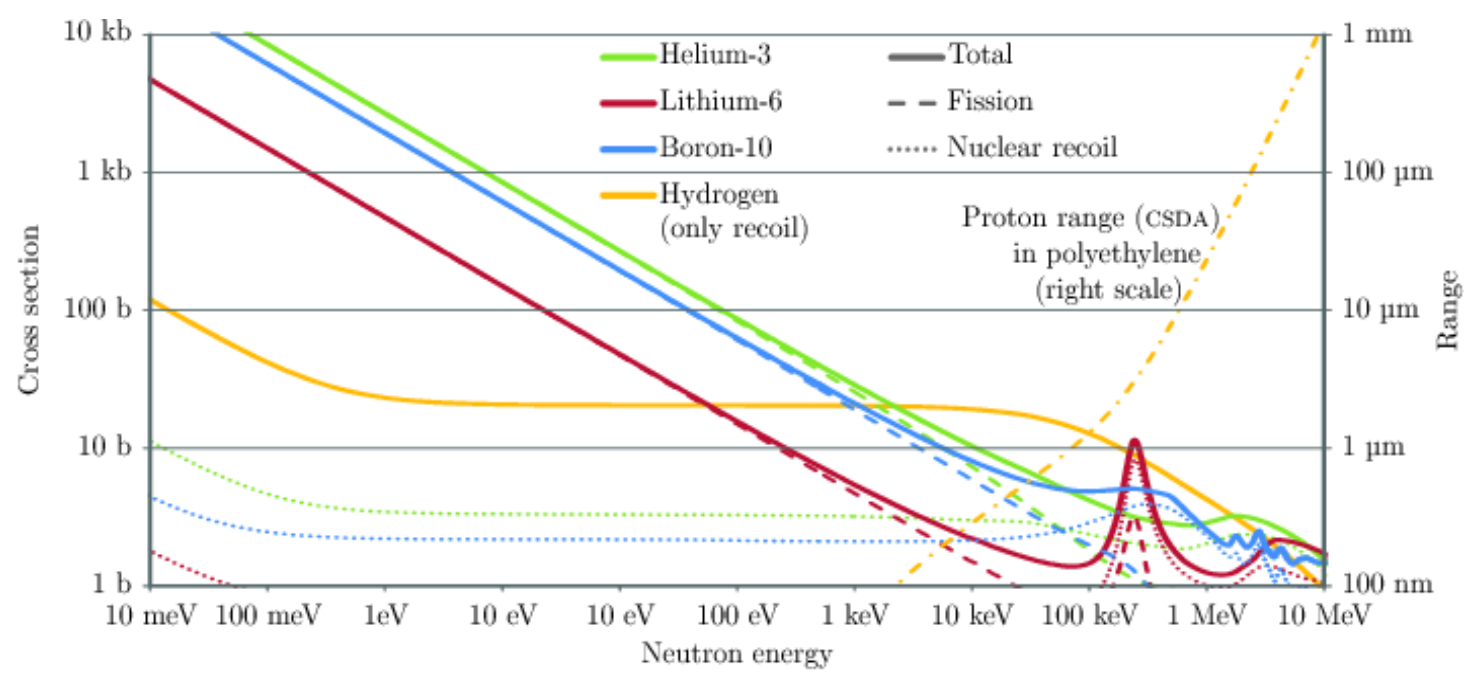

Figure 2-1: Neutron cross section for various materials used in neutron detectors[3]

The material used in neutron detectors can take the form of a solid, liquid or a gas, or a combination of both. Gas-filled detectors were one of the first detectors used for radiation counting. It takes about $30 \mathrm{eV}$ to create an ion pair in a gas, meaning a total of $\mathrm{E} / 30 \mathrm{eV}$ electron-hole pairs are created for a charge of kinetic energy $(E)$. The size of the pulse depends on the applied voltage, the detector geometry and the type of filling gas. Proportional counter is widely used with gas-filled neutron detector. They are used when extreme values of gas amplification, (A) is not required. A typical value of $\mathrm{A}$ is about $10^{3}$ for these counters. Proportional counters are typically filled with ${ }^{3} \mathrm{He}, \mathrm{BF}_{3}$ or $\mathrm{CH}_{4}$ gas at pressures of less 1 to about $20 \mathrm{~atm}$. The gas serves the purpose of both the target material and as the medium through which charged product nuclei losses energy.

Proportional counters operate at high voltages so that the primary charge particles can gain enough energy to ionize the gas and create secondary ionization. Small amounts of other gases (generally less than 5\%) such as argon are added to improve the detector performance. The presence of a heavy gas (such as argon) helps reduce the range of charge particles and improves the pulse height resolution and the detector dead time. Argon also increases the 
gamma ray sensitivity of these detectors, but a trade-off must be made when it is used. Quench gases are often added to control the ionization process.

Proportional counters typically consist of a cylindrical tube made from aluminum (copper or brass) with a wall thickness of about $0.3 \mathrm{~cm}$. The interior walls of these tubes are coated with activation charcoal to absorbed electronegative gases that build up during irradiation. These detectors generally use aluminum as a cathode because it has a small neutron cross section. The anode is always placed at the center of the detector. The wire is typically $0.03 \mathrm{~mm}$ thick gold-plated tungsten. Tungsten is used because it has the highest tensile strength at temperature above $1650^{\circ} \mathrm{C}$, the highest melting point and lowest vapor pressure of all metals and it is also resistant to corrosion. The choice of using gold is because of its good electrical conductivity.

The sensitivity of neutron detectors to gamma rays is an important criterion when choosing a neutron detector because neutrons are often associated with significant gamma ray events. Thermal neutron detectors turn to be less sensitive to gamma radiation than fast neutrons. This is due to the fact that the neutron cross section of materials used in thermal neutron detectors turns to favor more neutron absorption than gamma absorption. Table 2-1 shows the probability of interaction of typical gases. 
Table 2-1: Gamma Ray Interaction Probability for Different Detectors[4]

\begin{tabular}{|c|c|c|c|}
\hline $\begin{array}{l}\text { Choice of Detector } \\
\text { material }\end{array}$ & $\begin{array}{l}\text { Probability of } \\
\text { thermal } \\
\text { neutron } \\
\text { interaction }\end{array}$ & $\begin{array}{l}\text { Probability of } \\
\mathbf{1} \mathbf{M e V} \text { gamma } \\
\text { interaction }\end{array}$ & $\begin{array}{l}\text { Ratio of neutron to } \\
\text { gamma interaction } \\
\text { probability }\end{array}$ \\
\hline${ }^{* 3} \mathrm{He}(2.5 \mathrm{~cm}$ diam, $4 \mathrm{~atm})$ & 0.77 & 0.0001 & 7700 \\
\hline${ }^{*} \mathrm{BF} 3(5 \mathrm{~cm}$ diam, 0.66 & 0.29 & 0.0006 & 483.3 \\
atm $)$ & 0.01 & 0.001 & 10 \\
\hline${ }^{+4} \mathrm{He}(5 \mathrm{~cm}$ diam, $18 \mathrm{~atm})$ & 0.78 & 0.26 & 3 \\
\hline${ }^{+}$Scintillator $(5.0 \mathrm{~cm}$ thick $)$ & \multicolumn{2}{c}{} \\
\hline
\end{tabular}

* thermal neutron detector

+ fast neutron detector

The infuence of gamma rays in neutron detectors is due to the fact that gamma rays interact with the detector wall producing electrons which ionizes the detector gas. The pulses produced from electrons could sometimes be comparable to those from neutrons. In principle, it is very unlikely that the pulse from gamma radiation are larger than that from neutrons because most neutron detectors are designed to trap charge particles produced from neutron interaction which generally have a shorter range than electrons. The magnitude of the pulse produced from gamma makes it possible for detectors to employ a simple differential discrimination or single channel analyzer (SCA) to discriminate between neutrons and photons. The discrimator allows for the logic output logic pulse to be produced only if its input signal exceeds a given preset threshold level. The SCA could be set up such that it produces a logic output pulse only when the amplitude of the input signal is within two preset threshold values called the pulse-height window. The amplitude of the signal is proportional to the charge or energy of the detected events. Figure 2-2 shows an example of three different pulses that can be generated from an amplifier and sent to SCA. The upper level and the lower level of of discrimination is shown on Figure 2-2. As shown in the figure, only the pulse signal B satisfies the condition required to produce an output signal. The ouput of B is shown in the lower part of the figure. The above technique is very useful when separating events with different energies. The SCA can also be used as an integral 
discriminator by removing the upper-level of discrimination. In this case, signal A and B produce an output signal.

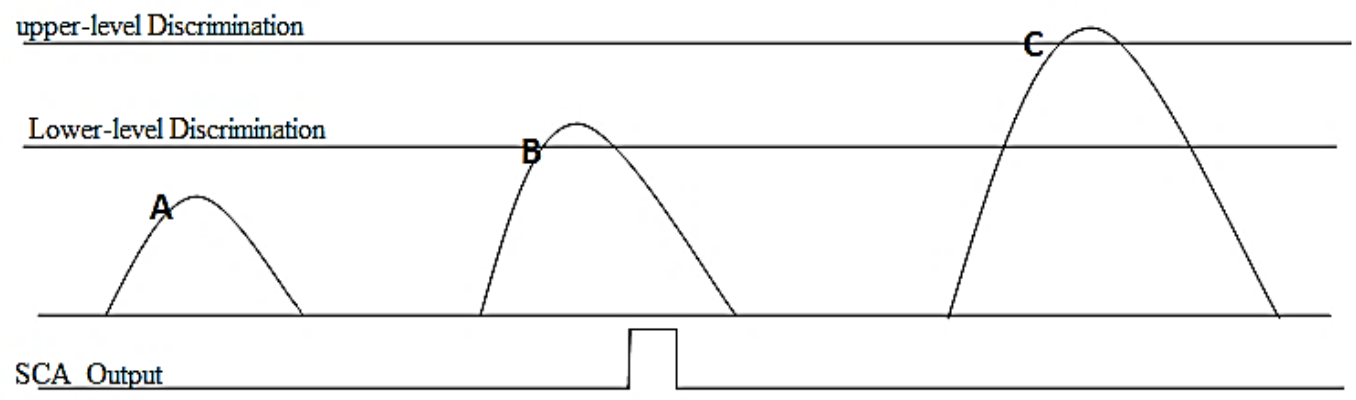

Figure 2-2: Single channel Analyzer

The detector efficiency is relevant in neutron detection. The cross-section variations, the gas pressures, detector geometry and the surrounding interferences affect the efficiency of neutron detectors. The detector sensitivity to gamma rays can be reduced by shielding the detector with a few centimeters of lead. It is also possible to use pulse height discrimination in some detectors. Sometimes detailed computational transport analysis is needed to analyze the functionality of these detectors with different radiation field. This approach requires the use of neutron transport codes such as MCNPX[5], SCINFUL[6] or Geant4[7] to determine the performance of these detectors.

The choice of detectors depends on the type of information needed about the neutron event. In slow neutron detectors, the information about the neutron energy is generally lost. Slow neutron detection is therefore desired when the goal is to determine the total neutron present. Fast neutron detection, on the other hand, is used when information about the energy of the neutron is desired. 


\subsection{Slow Neutron Detection}

Neutrons with energy below the cadmium cut-off of $0.5 \mathrm{eV}$ belong to the slow neutron region. At low neutron energies, most materials have a sensitivity that varies with the reciprocal of the velocity of the neutron. Slow energy neutrons are generally detected indirectly from the absorption reactions that occurs when neutron interacts with a target material. The typical material of choice are absorber materials with high neutron absorption cross sections. Typical materials used for slow neutron detection include helium-3, lithium-6, boron-10, and uranium-235. All slow neutron interactions are exothermic reactions leading to the release of charge particles and kinetic energy. Possible reaction products from slow neutron reaction are given below:

$$
\text { target nucleus }+ \text { incident neutron } \rightarrow\left\{\begin{array}{c}
\text { proton, or } \\
\text { alpha particle, or } \\
\text { fission fragment and } \\
\text { recoil nucleus. }
\end{array}\right.
$$

One important characteristic of this reaction is that the kinetic energy of the product depends solely on the excess energy liberated (Q-value) following the neutron capture reaction since the kinetic energy of the incoming neutron is negligible compared to the Q-value. It is important that this Q-value for a given reaction be as large as possible so that the gamma rays can easily be discriminated from neutrons.

The reaction products and the energy release are of great importance during neutron interaction. The size of the detector used in designing these detectors is dependent on the range of charged particle products. For small size detectors, some of the detector pulses resulting from the energy deposition from the reaction products are deposited in the gas medium meanwhile some fraction is deposited in the detector wall. As a result, a low energy continuum is introduced in the pulse. This phenomenon is called "wall effect". The "wall effect" can be minimized by constructing detectors with volumes large enough to stop the 
reaction products. In this case, all the energy of the nuclear product will be deposited within the gas volume and a response function will be a single full-energy peak as shown in Figure 2-3. Increasing the gas pressure in the detector can significantly reduce the "wall effect". However, this method turns to increase the detector sensitivity to gamma rays.

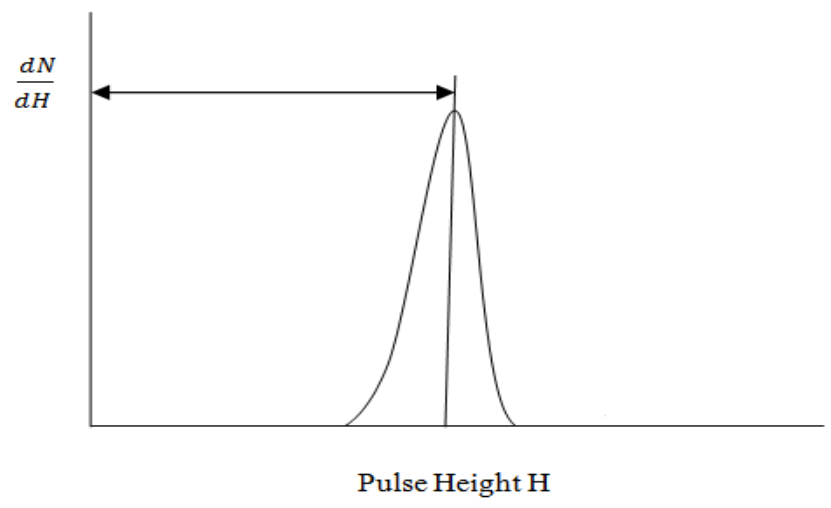

Figure 2-3: Single Peak Response Function of a neutron

In slow neutron detectors, the information about the energy of the neutron is lost because of the type of interaction involved. The energy recorded in the detector is the reaction energy and the remaining energy of the incident neutron. Hence, slow neutron detectors can only provide the information about the total neutrons detected but not the energy of the neutrons. A variety of detector exists for detecting slow energy neutrons. We will limit our discussion on the detectors that are of interest to our study.

\subsubsection{Helium-3 Detectors}

Helium-3 gas is the most widely used gas in neutron detectors. The natural abundance of ${ }^{3} \mathrm{He}$ in helium is $1.37 \mathrm{ppm}$. It has a thermal neutron cross section of 5316 barns at $2200 \mathrm{~m} / \mathrm{sec}$ and a scattering cross section for slow neutrons is 0.8 barns. ${ }^{3} \mathrm{He}$ has a high thermal neutron detection efficiency and a low gamma ray interaction probability, which makes it a very useful for neutron detection. A helium-3 detector with diameter of $2.54-\mathrm{cm}$ 
pressurized at $4 \mathrm{~atm}{ }^{3} \mathrm{He}$ has an efficiency of $77 \%$ for thermal neutrons $(0.025 \mathrm{eV})$. This makes ${ }^{3} \mathrm{He}$ detectors the choice for applications where high detector efficiency is needed. Other benefits of ${ }^{3} \mathrm{He}$ include good discrimination of gamma signal, inflammability, nontoxic, robustness and long operational life time[8]. This gas is produced artificially from tritium, which has a half-life of 12.3 years by the following reaction:

$$
{ }_{\mathbf{1}}^{3} \mathrm{H} \rightarrow{ }_{2}^{3} \mathrm{He}+{ }_{-1}^{\mathbf{0}} \boldsymbol{\beta}+{ }_{\mathbf{0}}^{\mathbf{0}} \overline{\mathbf{v}}+\mathbf{1 8 . 6} \mathbf{k e V} \quad \text { Equation } 2
$$

The greatest advantage of using ${ }^{3} \mathrm{He}$ detectors is that they can function in broad neutron range (fast and slow neutron regime). Its small atomic weight ensures significant transfer of energy from neutrons during elastic scattering making it useful for fast neutron detection. However, ${ }^{3} \mathrm{He}$ detectors are often used in thermal neutron detection because of its huge neutron cross section at thermal energy. The disadvantage of using ${ }^{3} \mathrm{He}$ is that ${ }^{3} \mathrm{He}$ is currently limited in supply and its cost is relatively high compared to boron fluoride detectors[9].

${ }^{3} \mathrm{He}$ detection is based on the reaction given below:

$$
{ }_{2}^{3} \mathrm{He}+{ }_{\mathbf{0}}^{\mathbf{1}} \mathbf{n} \rightarrow{ }_{\mathbf{1}}^{\mathbf{3}} \mathbf{H}+{ }_{\mathbf{1}}^{\mathbf{1}} \mathbf{p}(\mathrm{Q} \text {-value }=0.764 \mathrm{MeV}) \quad \text { Equation } 3
$$

The above reaction leads to the production of a triton, ${ }_{1}^{3} \mathrm{H}$ and a proton, ${ }_{1}^{1} p$ both released in opposite direction. The triton and proton share the $0.764 \mathrm{MeV}$ Q-value with the proton taking $0.573 \mathrm{MeV}$ and triton taking $0.191 \mathrm{MeV}$ of the total energy. When the reaction occurs close to the detector wall, one of the reaction products (either the proton or the triton) ends up being absorbed in the wall of the detector and the other product deposits its kinetic 
energy in the gas. The low Q-value makes it difficult for ${ }^{3} \mathrm{He}$ detectors to discriminate between gamma radiations.

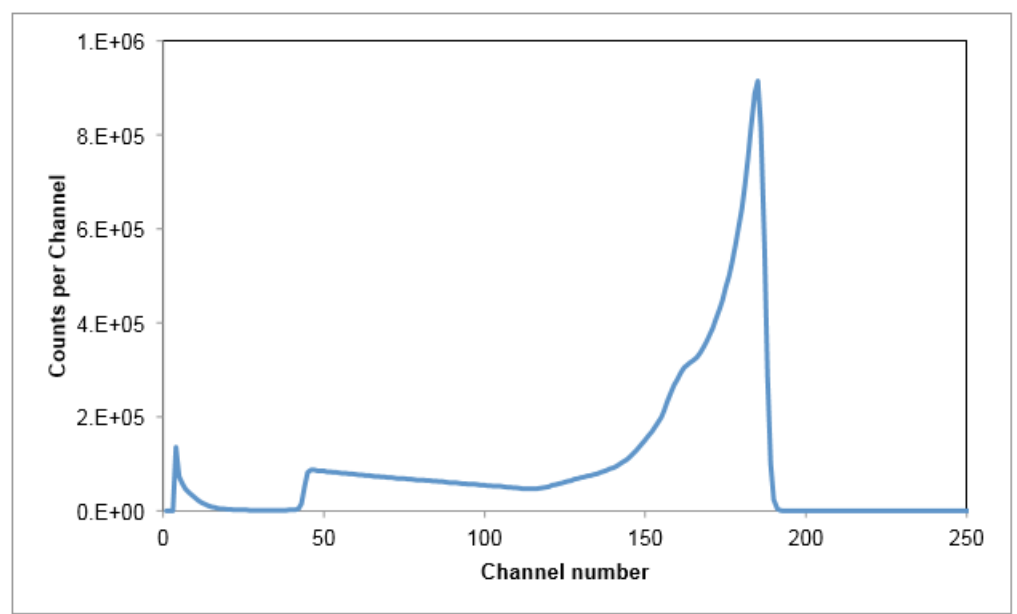

Figure 2-4: Pulse Height Spectra of He-3 Detector[10]

The pulse height spectrum of the ${ }^{3} \mathrm{He}$ detector is shown in Figure 2-4. The main neutron peak at $765 \mathrm{keV}$ (channel number 170) corresponds to both proton and triton being absorbed in the detection gas. On the pulse height spectrum, there are regions with discontinuous steps. This is because the range of protons and tritons, which have discrete energies, is larger than the dimension of the detector. When one of the reaction products collides with the wall of the detector, it dissipates its energies and does not contribute to the full energy peak, thereby creating discontinuous steps in the spectrum. The low pulse height noise to low energy electrons that are knocked off by gamma rays absorbed in the detector walls.

\subsubsection{Boron Neutron Detectors}

The element boron is not a gas; hence, proportional counters using the boron type neutron detectors use boron trifluoride $\left(\mathrm{BF}_{3}\right)$ gas as a proportional gas. Natural boron contains $80.2 \%{ }^{11} \mathrm{~B}$ and $19.8 \%{ }^{10} \mathrm{~B}$. The gas is highly enriched to $96 \%{ }^{10} \mathrm{~B}$ to increase its 
efficiency. ${ }^{10} \mathrm{~B}$ has a cross section of 3843 barn at $2200 \mathrm{~m} / \mathrm{sec}$ and a scattering cross section for slow neutrons is 1.4 barns. This detector is mostly used for slow neutron detection because boron is readily available [11-16]. $\mathrm{BF}_{3}$ detectors are stable and have lower detector efficiency for thermal neutrons than ${ }^{3} \mathrm{He}$ detectors. The detection efficiency for an ideal 276 $\mathrm{nm}$ thick $\mathrm{B}-\mathrm{C}$ detector is $1.3 \times 10^{-3}$ [12]. The gamma ray sensitivity is slightly better than that of ${ }^{3} \mathrm{He}$ for gamma radiation. The reaction of interest for slow neutron detection is given below.

$$
{ }_{5}^{10} B+{ }_{0}^{1} n \rightarrow\left\{\begin{array}{c}
{ }_{3}^{7} L i+{ }_{2}^{4} \alpha+2.79 \mathrm{MeV} \quad(B R 6 \%) \\
{ }_{3}^{7} L i^{*}+{ }_{2}^{4} \alpha+2.31 \mathrm{MeV}(\mathrm{BR} 94 \%)
\end{array}\right.
$$

The reaction leads to the release of lithium-7 and an alpha particle both travelling in opposite direction and sharing the reaction Q-value. The Q-value is proportional to the relative masses of the products. Since the reaction leads to two pathways, the size of the resulting pulse would depend on whether the lithium nucleus produced is in the ground state (Q-value of $2.792 \mathrm{MeV}$ ) or an excited state (Q-value of $2.310 \mathrm{MeV}$ ). For the leading branch, $94 \%$ of the neutron capture reaction leads to an excited state with $\mathrm{Q}=2.310 \mathrm{MeV}$, resulting to $0.84 \mathrm{MeV}$ and $1.470 \mathrm{MeV}$ for the initial kinetic energies (KEs) of the ${ }^{7} \mathrm{Li}$ and ${ }^{4} \mathrm{He}$, respectively. The excited state decays to the ground state by emitting a $478 \mathrm{keV}$ gamma ray. For the less dominant branch, $6 \%$ of the neutron capture reaction leads to the ground states $(\mathrm{Q}=2.792 \mathrm{MeV})$, producing higher initial energies of $1.015 \mathrm{MeV}$ and $1.777 \mathrm{MeV}$, respectively.

The information about the incoming energy is lost because the Q-value in either case is larger than the energy of the incoming neutron. Figure 2-5 shows the typical pulse height spectrum from a $\mathrm{BF}_{3}$ detector. Two steps are created on the left of the $2.31 \mathrm{MeV}$ peak as 
result of the "wall effect". The contribution from ${ }^{4} \mathrm{He}$ can be seen throughout the spectrum because it has a higher maximum kinetic energy. ${ }^{7} \mathrm{Li}$ has a smaller maximum kinetic energy and, so it contributes only to the lower region of the spectrum. The sum of ${ }^{4} \mathrm{He}$ and ${ }^{7} \mathrm{Li}$ is therefore what produces the double-plateau shape. The observed pulse height spectrum is a function of the detector construction, i.e. size and geometry of the detector and is independent of the neutron energy. In this detector, it is mostly the count rate that carries useful information. $\mathrm{BF}_{3}$ detector differs from the ${ }^{3} \mathrm{He}$ detector in that $\mathrm{BF}_{3}$ gas is polyatomic, so $\mathrm{BF}_{3}$ detectors operate at higher voltages than ${ }^{3} \mathrm{He}$. Operating voltage of this detector usually varies from 2000-2500V. Because of the neutron cross section of ${ }^{10} \mathrm{~B}$, the size of $\mathrm{BF}_{3}$ is usually larger. $\mathrm{BF}_{3}$ neutron detectors are not widely used in neutron detection because of the safety issues with the $\mathrm{BF}_{3}$ gas since the gas is highly toxic. The boron-lined gas-filled proportional counters are also used in neutron detection. The method of detection is similar to that of the $\mathrm{BF}_{3}$ gas filled proportional detectors except that the walls of the counter are coated with ${ }^{10} \mathrm{~B}$. The boron-lined counter has a moderate detector efficiency for thermal neutrons and a low detection efficiency for fast neutrons. The efficiencies of these detectors are about seven times less efficient than ${ }^{3} \mathrm{He}$ detectors. They are better suited in situations were lower efficiency is not problematic.

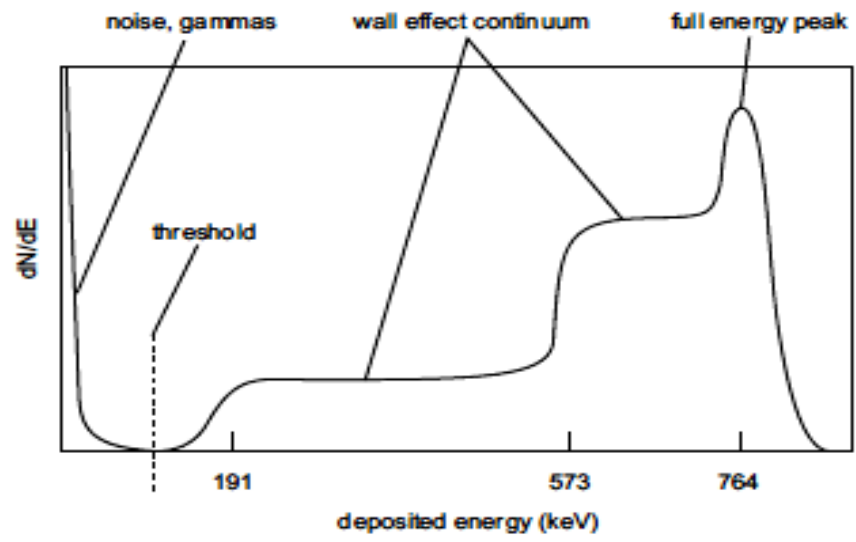

Figure 2-5: Expected pulse height spectrum from $\mathrm{BF}_{3}$ detector[ https://www.orau.org/ptp/collection/proportional\% 20counters/bf3info.htm] 


\subsubsection{Lithium-6 Neutron Detectors}

Another method of detecting neutrons involves $(\mathrm{n}, \alpha)$ reaction in ${ }^{6} \mathrm{Li} .{ }^{6} \mathrm{Li}$ have a thermal neutron cross section of 940 barns at $2200 \mathrm{~m} / \mathrm{sec}$ and a scattering cross section for slow neutrons is 1.4 barns. The natural abundance of lithium- 6 is $7.40 \%$. The reaction of interest for neutron detection in ${ }^{6} \mathrm{Li}$ is given below.

$$
{ }_{3}^{6} \mathrm{Li}+{ }_{0}^{1} \mathrm{n} \rightarrow{ }_{1}^{3} \mathbf{H}+{ }_{2}^{4} \alpha
$$

Equation 4

The Q-value for this reaction is $4.78 \mathrm{MeV}$ which is shared between tritium and alpha with the alpha particle carrying $2.05 \mathrm{MeV}$ of the total energy. The alpha particles and the triton are emitted in opposite direction. The Q-value of $(\mathrm{n}, \alpha)$ in Li-6 is very large making these detectors suitable for efficient discrimination against gamma rays. A lithium proportional counter does not exist because no lithium containing stable gas exists. As a result, Li-6 based detectors used in thermal neutron detection take advantage of scintillation process to detect the reaction products. The lithium is loaded as a substrate inside a scintillator. The scintillation mechanism is similar to that employed by NaI detectors using Lithium iodide crystals. Europium (less than 0.1 ) is added in these detectors to increase the light output by about $35 \%$. Such output is comparable to light outputs obtained with NaI detectors. The crystal size is generally made large enough such that the reaction products deposit all its energies. The pulse height spectrum from these detectors is a single peak free from "wall effects." 


\subsection{Fast Neutron Detection}

The mechanism of detecting fast neutrons is different from that of slow neutrons. Slow neutron detectors have a very low efficient for fast neutron detection because their capture cross section is too small. The low capture cross section makes it difficult for a fast neutron to hardly interact with the detection gas. The low efficiency for fast neutron detection can be improved by surrounding the detector with a moderator. Typically, $10 \mathrm{~cm}$ ( 4 inch) polyethylene surrounds the detector. The moderator allows the fast neutrons to be slowed down to thermal energies before interacting with the detector[17]. During this process, the information about the neutron energy, the direction of travel and the time of emission is lost. The energy recorded in the detector is the reaction energy and the remaining energy of the incident neutron.

The most common method of detecting fast neutron is through elastic scattering of light nuclei, resulting in the formation of recoil proton and a recoil nucleus. In recoil detectors, the neutron is detected without initial thermalization, thus the initial energy of the neutron is preserved. When detecting fast neutrons, the detection threshold of the counting system is set high enough to reject low level noise and gamma rays but without compromising the detection of intermediate and high energy neutrons. The energy of the secondary particle depends significantly on the neutron energy. The raw pulse height spectrum is a combination of neutrons, gammas, and wall effects. The neutron energy can determine by unfolding the spectrum. Fast neutrons detectors are widely used in applications where the information about the energy of the neutron energy is important. For nuclei of atomic weight, A, the recoil energy $E_{r e c}$ of the nuclei is related to the incident energy $E_{n}$ by the relationship

$$
E_{\text {rec }}=E_{n} \frac{4 A}{(1+A)^{2}} \cos ^{2} \emptyset
$$

Equation 5 
Where $\varnothing$ denotes the angle of recoil emission in the lab frame. The maximum energy transfer is given by:

$$
E_{\max }=E_{n} \frac{4 \mathrm{AE}}{(1+A)^{2}}
$$

The above equation shows that the maximum energy transfer occurs when $A=1$. Because the maximum energy transfer occurs when the atomic mass of the target material which collides with a neutron is comparable to the neutron mass, hydrogenous materials are often the preferred target nuclei for fast neutron detection. An incoming neutron could transfer up to its maximum energy to hydrogen in a single collision. In elastic scattering, the Q-value is zero because the total kinetic energy after the reaction is the same as the total kinetic energy after the reaction. For a single scattering event in hydrogen, the energy transfer to the recoil nucleus varies from 0 to $E_{\text {max }}$ depending on the angle. The raw pulse height spectrum from these detectors is a combination of neutron spectra due to the neutron source, the recoil energy ranging from zero to full neutron energy $\left(E_{\max }\right)$, gamma ray pile-up events, noise, and scattered events. For this reason, even for bombardment with monoenergetic neutrons, a broad pulse height spectrum is obtained. Thus, the neutron convoluted within the pulse height spectrum must be separated. This is done by unfolding the raw spectra. The process of unfolding detector spectra will be discussed later.

Examples of fast neutron detectors include: proton recoil detector, liquid scintillators, fission chambers, ${ }^{4} \mathrm{He}$ based noble gas detectors and plastic detectors. 


\subsubsection{Neutron Scintillation Detection}

Scintillation neutron detectors include liquid organic scintillators, crystals, plastics, and glass. A scintillator counter consists of a photomultiplier tube to which is fixed a scintillator. Scintillations are used in fast neutron response because of their fast time response. The mechanism of operation involves the absorption of the kinetic energy of the recoil protons and subsequent conversion into heat and visible light using a scintillating material. The light can then be collected by photomultiplier tube which converts the light into an electrical pulse. The size of the pulse is related to the incident energy. A good material for neutron detector scintillation is supposed to be transparent to its own radiation, easily converts recoil protons to a fluorescent radiation, and has a good optical coupling of photomultiplier tube with the scintillator.

Neutron scintillators are very sensitive to gamma ray because of the high density of gases used in the scintillation detectors. From the fast neutron detector standpoint, these detectors are not as good as proportional counters because of the light outputs from electrons and gamma rays which is far larger than the light output that can be obtained from alpha and protons with the same energy. The detection probability for gammas and neutrons are often comparable and the pulse height distributions are broad and overlap. In some liquid scintillators detectors, fast neutrons produce scintillations with different decay of neutrons and gammas. Figure 2-6 shows the pulse height discrimination of fast neutrons on EJ309 detector from plutonium beryllium source $(\mathrm{PuBe})$. The different radiation types can be distinguished using pulse shape discrimination techniques. This generally affects the efficiency of these detectors. The efficiency of neutron absorption in liquid scintillators can be improved by adding $0.5 \%$ by weight of gadolinium to the liquid. Examples of neutron scintillation include NE101 and NE213. 


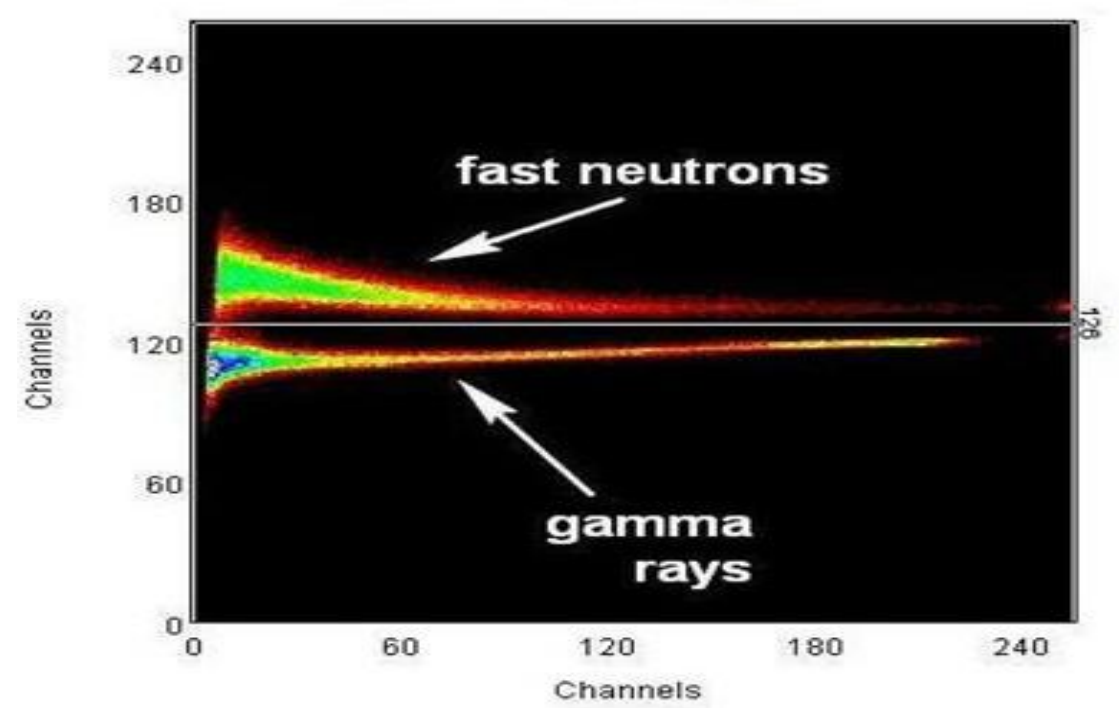

Figure 2-6: Pulse Height Discrimination of fast neutrons on EJ309 detector from PuBe [http://www.johncaunt.com/detectors/scintillation-detectors/2307-2/]

\subsubsection{Proton Recoil Proportional Counter}

Proton recoil proportional counters are either spherical or cylindrical. Spherical proportional counters have a near isotropic response; hence, no knowledge of the direction of the neutron is required[18]. One problem with the proton recoil detectors is their limited energy range. The proton recoil proportional counters have been used in neutron spectrometers within the energy range from a few tenths of keV up to a few $\mathrm{MeV}$. Proportional counters have a low detection efficiency because the medium of detection is a gas with relatively low density. The capture cross section of hydrogen is 0.328 barns at 2200 $\mathrm{m} / \mathrm{sec}$. Typical efficiencies are less than $1 \%$. Gas proportional counters are also less sensitive to gamma rays compared to scintillation counters because typically the range of gamma ray produced by recoil electrons is smaller than the dimension of the detectors [19-21]. It is expected that these counters have good energy resolution and should produce pulses with a fast rise time. The shape of the pulse height distribution in these counters is expected to be rectangular. However, because of incomplete energy deposition from proton escaping the 
detector volume ("wall effects"), as well as the energy dependence of the W-value needed to create an electron-hole pair, the shape of the spectrum is modified.

Spherical hydrogen proportional detectors are commonly used in fast neutron counting. The Rotating Spectrometer, ROSPEC used in the NIST facility[22] is a good example of a detector assembly that uses proton recoil detectors. The ROSPEC consists of a set of different detectors used for detecting neutrons from bare and moderated ${ }^{252} \mathrm{Cf}$, monoenergetic neutron fields of $2.5 \mathrm{MeV}$ and $14 \mathrm{MeV}$, and a thermal-neutron beam giving good approximation of the neutron spectrum. The ROSPEC has ability to measure neutron spectra from $0.01 \mathrm{eV}$ to $17 \mathrm{MeV}$ with the spectrum above $50 \mathrm{keV}$ having resolution of $10 \%$ to $15 \%$. The ROSPEC consists of seven different configurations, four of which is gas-filled proton recoil counter, which measure the neutron spectrum from $50 \mathrm{keV}$ to $4.5 \mathrm{MeV}$. There are two ${ }^{3} \mathrm{He}$ detectors that measure neutron energy from $0.01 \mathrm{eV}$ to $50 \mathrm{keV}$ and a plastic scintillators proton-recoil detector, which measures the spectrum from $4 \mathrm{MeV}$ to $17 \mathrm{MeV}$. Figure 2-7 shows the ROSPEC. The largest detector, SP-6, is on the right side. When in operation, the detector rotates about an axis through the center of a cylinder at one revolution per minute. The energy range of the detectors overlaps given room for verification of detector results. The characteristics of the different proton recoil detectors used in the ROSPEC are shown in Table 2-2. The neutron spectra from the proton recoil data are unfolded from the measured data. This is done using the Spec-4 computer code. 


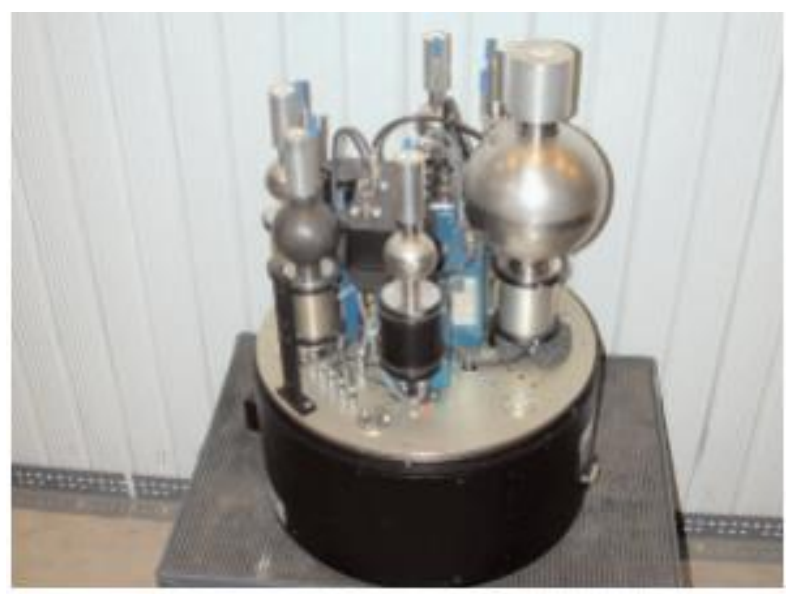

Figure 2-7: ROSPEC Detector

Table 2-2: ROSPEC Detector Characteristics

\begin{tabular}{|l|l|l|l|l|}
\hline Detector & Radius(m) & Fill Gas & Pressure(kPa) & Energy Range \\
\hline SP2-1 & 2.54 & $\mathrm{H}_{2}$ & 76 & $0.05 \mathrm{MeV}-0.25 \mathrm{MeV}$ \\
\hline SP2-4 & 2.54 & $\mathrm{H}_{2}$ & 400 & $0.15 \mathrm{MeV}-0.75 \mathrm{MeV}$ \\
\hline SP2-10 & 2.54 & $\mathrm{H}_{2}$ & 1000 & $0.4 \mathrm{MeV}-1.5 \mathrm{MeV}$ \\
\hline SP6 & 7.62 & $\mathrm{CH}_{4} / \mathrm{Ar}$ & 500 & $1.2 \mathrm{MeV}-5.0 \mathrm{MeV}$ \\
\hline
\end{tabular}

\subsubsection{Neutron Unfolding}

Neutron detection from proton recoil detectors occurs from the recoil protons produced from neutron-hydrogen collision. Electrons are generated from gamma-ray interaction within the counter walls. The resulting gamma rays can be discriminated by removing all data below the maximum electron pulse. The main contribution to the pulse height spectrum in neutron interaction is the so called "wall effect," tail effect and "end effect." The wall effects results from the collision between the recoil proton and the inner wall of the cathode. The tail effect occurs from recoil proton hitting the non-sensitive zones in the detector, while the end effect results from recoil proton going out of the sensitive zone. 
Accurate knowledge of the detector spectrum requires unfolding of the pulse height spectrum. The differential pulse height spectrum $d N / d H$, the detector response $R(H, E)$, and the neutron energy spectra $\phi(E)$ are related by the Fredholm integral equation given below

$$
\frac{d N}{d H}=\int_{E_{\min }}^{E_{\max }} R(H, E) \phi(E) d E
$$

The component $d N / d H$ is directly obtained from the radiation detector, which is as a result of folding the response function and the energy distribution of the energy spectrum. The response function can be calculated accurately because the kinematics of a n-p scattering is well understood.

Equation 10 can be written in a discrete form as

$$
\begin{array}{ll}
\boldsymbol{N}_{\boldsymbol{i}}=\sum_{i} \boldsymbol{R}_{\boldsymbol{i j}} \boldsymbol{\phi}_{j} & \text { Equation } 8
\end{array}
$$

Where, $N_{i}$ is the count in the $i^{\text {th }}$ channel $R_{i j}$ is the response matrix and $\phi_{j}$ is the radiation fluence in the $j^{\text {th }}$ energy interval. This representation is essential when the detector output is recorded using a multi Chanel analyzer. The above equation has to be inverted so as to obtain the neutron spectrum from the measured pulse height distribution. Various methods have been proposed in unfolding neutron spectra: least square fit [23], iterative [24], the Monte Carlo method[25], and more recently the neutral network[26]. There are a number of codes used for this purpose. The Spec-4 [27] computer has been used to solve spectrum unfolding problems for spherical gas-filled proton recoil neutron spectrometers. The unfolding technique is achieved by calculating the proton-recoil energy distributions from mono-energetic neutrons, and then unfold the measured proton-recoil spectra to yield the corresponding continuous energy spectra. The required input of the program includes the measured proton recoil spectrum, the relevant counter parameters, and an estimate of the 
neutron spectrum above the highest measured energy. Due to the near-rectangular distribution of proton recoils, even neutrons with energies greater than the detection limit of the gas counter will introduce some noise signals in the detector. As a result, the Spec-4 code requires an estimate of the neutron flux above the upper limit of the counter to remove the undesired portion of the portion of the measured data. The value of this upper limit can be determined by visual examination of the raw data. The code was originally built for spherical, gas-filled proton recoil proportional counters called the Benjamin Counters. Two counters were used for the initial analysis, SP-28, which is filled with pure hydrogen gas at a pressure of 10 atms and is used for higher energy end of the spectrum, and SP-24 is filled with a pressure of 3 atms with a mixture of $85 \%$ hydrogen and $10 \%$ methane and $5 \%$ nitrogen. These detectors contained an alpha source deposited on its collector wire. The alpha deposits a fixed amount of energy proton energy proportional to the pressure of the gas filled in the detector. The deposited energy divided by the centroid of the channel number of the centroid of the observed alpha peak gives the calibration of the detector.

The slow and fast neutrons used in most neutrons applications have been identified. The helium-3 detector is ideal for measuring neutron burst from titanium-deuterium because these detectors are very stable, can discriminate gamma radiation, has a high efficiency for thermal neutrons and are non-toxic. For measuring fast neutrons, the helium-3 detector is enclosed with a polyethene sleeve to slow down the fast neutrons. This comes as a cost, since the information about the neutron is distorted. The energy recorded in the detector is the reaction energy and the remaining energy of the incident neutron. The helium-3 detector is very useful if the purpose is to determine the neutron burst from experimental systems. The proton recoil detectors are desirable for fast neutron detection because they less sensitive to gamma ray scintillation detectors. The used of proton recoil detectors will require unfolding the detector spectra. 


\section{REFERENCES}

1. Breit, G. and E. Wigner, Capture of slow neutrons. Physical review, 1936. 49(7): p. 519.

2. $\quad$ Knoll, G.F., Radiation detection and measurement. 2010: John Wiley \& Sons.

3. Pinto, S.D., GEM applications outside high energy physics. Modern Physics Letters A, 2013. 28(13): p. 1340025.

4. Crane, T. and M. Baker, Neutron detectors. Passive Nondestructive Assay of Nuclear Materials, 1991: p. 379-406.

5. McKinney, G.W., et al., MCNPX overview. Proceedings of the 2006 HSSW, FNAL, IL, LA-UR-06-6206, 2006.

6. Dickens, J., SCINFUL: A Monte Carlo based computer program to determine a scintillator full energy response to neutron detection for $E / s u b \mathrm{n} /$ between 0.1 and 80 MeV: Program development and comparisons of program predictions with experimental data. 1988, Oak Ridge National Lab., TN (USA).

7. Allison, J., et al., Geant4 developments and applications. IEEE Transactions on Nuclear Science, 2006. 53(1): p. 270-278.

8. Simpson, A., et al. A Review of Neutron Detection Technology Alternatives to Helium-3 for Safeguards Applications. in INMM 52nd Annual Meeting. 2011.

9. Shea, D.A. and D. Morgan. The helium-3 shortage: Supply, demand, and options for Congress. 2010. Congressional Research Service, Library of Congress.

10. Rogers, J.L., et al., Neutron Coincidence Counting Studies. 2012, Technical Report PNNL-21686. Pacific Northwest National Laboratory, Richland, WA.

11. Wang, Z. and C.L. Morris, Multi-layer boron thin-film detectors for neutrons. Nuclear Instruments and Methods in Physics Research Section A: Accelerators, Spectrometers, Detectors and Associated Equipment, 2011. 652(1): p. 323-325.

12. Robertson, B., et al., A class of boron-rich solid-state neutron detectors. Applied physics letters, 2002. 80(19): p. 3644-3646.

13. Rose, A., Sputtered boron films on silicon surface barrier detectors. Nuclear Instruments and Methods, 1967. 52(1): p. 166-170.

14. Lintereur, A.T., et al., Boron Lined Neutron Detector Measurements. Technical Rpt. PNNL-18938, Pacific Northwest National Laboratory, Richland, WA, 2009.

15. Lacy, J.L., Boron coated straw neutron detector. 2006, Google Patents.

16. Harken, A.D., et al., Boron-rich semiconducting boron carbide neutron detector. Japanese journal of applied physics, 2005. 44(1R): p. 444.

17. Dunning, J., et al., Interaction of neutrons with matter. Physical Review, 1935. 48(3): p. 265.

18. Thomas, D.J., Neutron spectrometry for radiation protection. Radiation Protection Dosimetry, 2004. 110(1-4): p. 141-149.

19. Jansky, B. and E. Novak, Neutron spectrometry with spherical hydrogen proportional detectors. Nuclear Instruments and Methods in Physics Research Section A:

Accelerators, Spectrometers, Detectors and Associated Equipment, 2014. 735: p. 390398.

20. Pichenot, G., et al., Characterisation of spherical recoil proton proportional counters used for neutron spectrometry. Nuclear Instruments and Methods in Physics Research Section A: Accelerators, Spectrometers, Detectors and Associated Equipment, 2002. 476(1): p. 165-169.

21. Leake, J., An improved spherical dose equivalent neutron detector. Nuclear Instruments and Methods, 1968. 63(3): p. 329-332. 
22. Heimbach, C., NIST calibration of a neutron spectrometer ROSPEC. Journal of research of the National Institute of Standards and Technology, 2006. 111(6): p. 419.

23. Stallmann, F., LSL-M2: a computer program for least-squares logarithmic adjustment of neutron spectra. 1986, Oak Ridge National Lab., TN (USA).

24. Sanna, R., Manual for BON: a code for unfolding multisphere spectrometer neutron measurements. 1981, USDOE Environmental Measurements Lab., New York.

25. Sanna, R. and K. O'brien, Monte-Carlo unfolding of neutron spectra. Nuclear Instruments and Methods, 1971. 91(4): p. 573-576.

26. Vega-Carrillo, H.R., et al., Neutron spectrometry using artificial neural networks. Radiation Measurements, 2006. 41(4): p. 425-431.

27. Johnson, J. and D. Ingersoll, User's guide for revised SPEC-4 neutron spectrum unfolding code. 1980, Oak Ridge National Lab., TN (USA). 


\section{MATERIALS AND METHODS}

The experimental design used in this work involved four main components: the experimental chamber or thermal shock system, sample preparation, neutron detectors and the analysis tools. These four components will be described in this section.

\subsection{Description of the thermal Shock System}

Our thermal shock chamber consists of Swagelok tubes, pressure valves, two thermocouples, pressure gauges, a roughing pump and a diffusion pump. The Swagelok tubes were $3 / 8^{\prime \prime}$ and $1 / 4$ ' inch tubes [1] connected at different points to form a close system that was used for loading the titanium powder. The $3 / 8$ ' ' and $1 / 4$ ' ' tubes were connected to each other using reducers. Valves were used at different points to separate different areas of the system. These Swalog fittings can handle pressure of up to about $20000 \mathrm{psig}$. The thermal shock chamber was connected to a deuterium gas tank and a diffusion pump. A 3/4", particulate inline filter was used to trap the titanium powder from escaping the tube while the system was being evacuated. Two type " $\mathrm{K}$ " thermocouples were used to record temperatures. One of the thermocouple was inserted inside the thermal shock chamber to record the temperature of the titanium powder while the other thermocouple was placed outside the chamber to measure ambient temperature. A GE Druck DPI 104 digital pressure gauge was used to measure the pressure of deuterium in the chamber. The gauge is capable of measuring pressure ranging between 0 to 10000 psig with accuracy of $0.05 \%$ of its full scale. The experimental chamber is shown in Figure 3-1. 


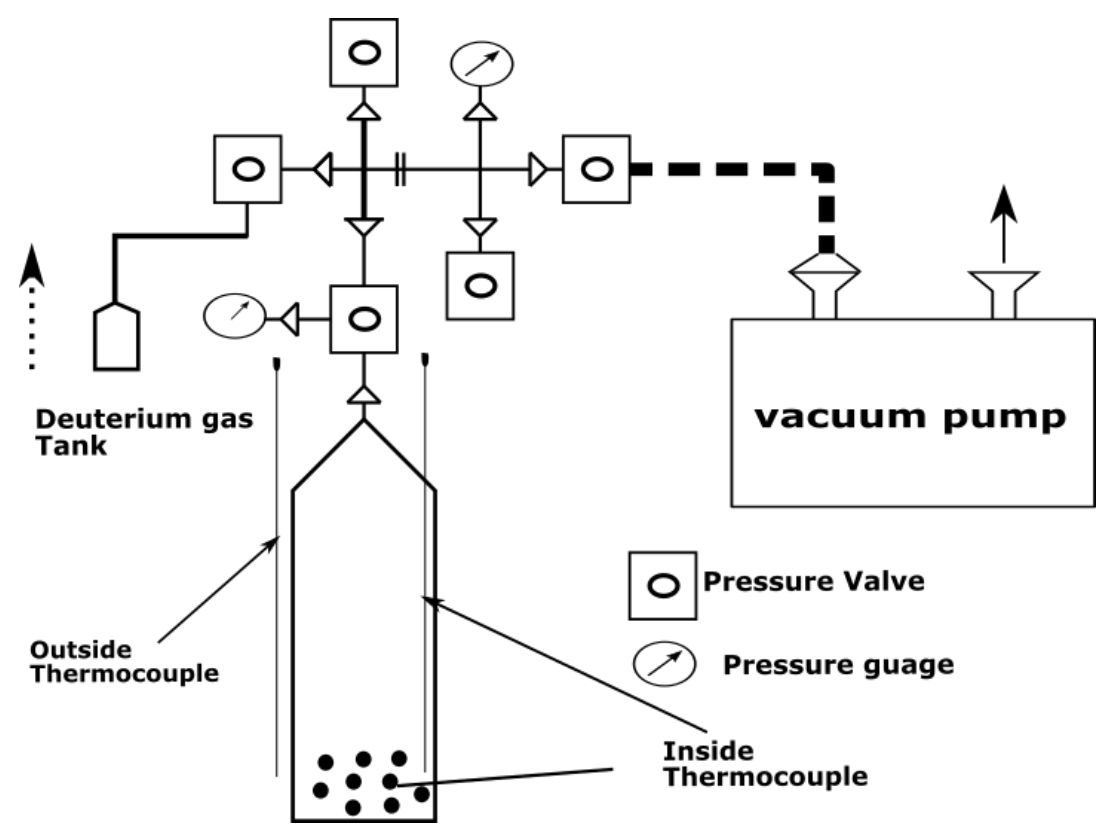

Figure 3-1: Experimental Chamber

\subsection{Sample Preparation}

The sample used in our experiments was dehydrided, -325 titanium mesh, 99.99\% purity which was commercially purchased from Alfa Aesar. This sample is $44 \mu \mathrm{m}$ in diameter. The powder was weighed with a scale balance and loaded into the experimental chamber in an argon atmosphere inside a glove bag. This procedure eliminated oxidation of the titanium powder. The chamber was then evacuated using a rotary pump followed by a diffusion pump to a pressure of less than $1 \times 10^{-6}$ torr, while at the same time baking the system at a temperature of $\sim 300^{\circ} \mathrm{C}$ using a heat tape. The baking procedure helped removed oxygen and other containments in the chamber. The presence of oxygen in the thermal shock chamber limits the deuterium-titanium reaction because oxygen is known to occupy sites on the titanium lattice through the formation titanium oxide thereby inhibiting the formation of titanium deuteride. 
The experiment chamber was leaked-tested with a helium-3 mass spectrometer to ensure that the integrity of the system was preserved before introducing deuterium gas into the chamber. The chamber was pressurized with deuterium gas $(99.96 \%)$ purity at room temperature with pressure of about 200 psig. The chamber was later placed in liquid nitrogen bath for six hours to ensure adequate deuterium loading and all counting systems was started to collect data. The data collection was done using a LabVIEW program. The LabVIEW program recorded the pressure, the inside temperature of the chamber, ambient temperature and the detector counts at intervals of $0.1 \mathrm{~s}$. After placing the system in liquid nitrogen, the pressure of the system dropped, which is in part due to a decrease in temperature of the experimental chamber, and because of titanium deuteride formation. The formation of titanium deuteride is exothermic, so there could be a temperature rise in the titanium sample which is also monitored. The experimental chamber is then rapidly transferred into boiling water bath at $100^{\circ} \mathrm{C}$.

\subsection{Neutron Detection}

Three neutron detectors were used in this work: 1 . An unmoderated helium-3 detector which responds to the capture of slow neutrons by helium-3 reaction; 2 . A helium-3 detector wrapped in 4 inches of polyethylene sleeve which responds to neutrons slow and fast neutrons; and, 3. A proton recoil detector which only responds to fast neutrons. The goal of using different detectors was to cover the range of neutron energies from thermal to fast. Helium-3 and proton recoil detectors are very stable detectors. Figure 3-2 shows the sensivity of the two different detectors used in this experiment. The bare helium-3 detector is sensitive to neutron energy up to about $10 \mathrm{eV}$; the moderated helium-3 is sensitive to all range of neutron energies. The two helium-3 detectors will be used to measure neutron energies below $10 \mathrm{eV}$. The proton recoil detector will be used to measure neutrons with energy greater than $60 \mathrm{keV}$. Both the moderated helium-3 and proton recoil detectors will pick up neutrons if 
their energy is above $60 \mathrm{keV}$. Hence, neutrons of any range will be detected by at least two detectors.

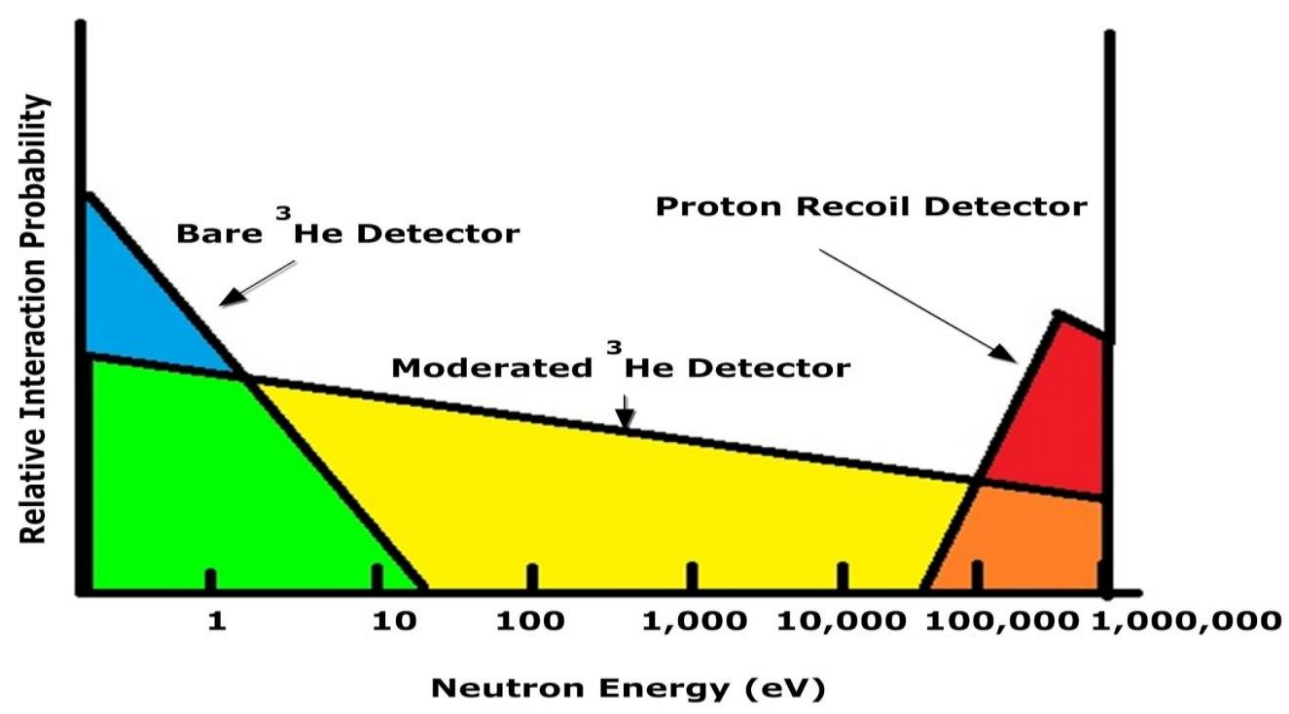

Figure 3-2: Sensitivity of neutron Detectors

Because of the nature of the experiment required a time stamp of neutron emission, a BNC T adapter was used to split the amplifier signal between the MCA and the SCA. The SCA output signal was sent to a LabVIEW acquisition system, where counts were collected every $100 \mathrm{msec}$. The time stamp from these measurements tells us when neutron bursts were produced during the experiment. Several long background counts were collected before running the experiment.

\subsection{Detector Calibration}

The neutron detectors were all initially calibrated with Cf-252 source at the University of Missouri Research Reactor (MURR). The neutron source later used to test the detectors was produced from the interaction of Po-210 source and a beryllium metal to prevent moving the detectors to MURR. The PoBe source consist of a beryllium metal sandwiched in a $5 \mathrm{mCi}$ Po-210 source which was obtained from Nuclespot ${ }^{\mathrm{TM}}($ Model P-2042[2]. The B (n, $\alpha)$ reaction 
is an important reaction because it leads to the production of neutrons and an excited state in the ${ }^{12} \mathrm{C}$ nucleus[3]. Po-210 emits alpha radiation with energy of 5.4 MeV. SRIM [4] calculations shows that the range of $5.4 \mathrm{MeV}$ alphas in beryllium metal is about $27.8 \mathrm{um}$ as shown in Figure 3-3. The beryllium metal used for this work had a dimension of $5.2 \times 3.6 \times 2.6 \mathrm{~cm}$. The beryllium metal is thick enough to stop all alphas from Po-210. The probability of this reaction occurring depends on the threshold energy and the height of the Columbic barrier. Neutrons are emitted in a wide range of spectrum. The maximum neutron energy expected from ${ }_{4}^{9} \mathrm{Be}(n, \alpha)$ is about $10.8 \mathrm{MeV}$ and average neutron energy of $4.2 \mathrm{MeV}$ for a 5.2 MeV alpha particle[5]. The neutrons produced are slowed down by a moderator medium before reaching the helium-3 detector. Neutrons were emitted as well as gamma ray. Under the experimental condition, the background counts are small compared to the neutrons resulting from the interaction of beryllium with alpha. This method of producing neutrons has been used in the past for neutron counting at the Los Alamos National Laboratory[6]. The interaction between the beryllium source and the Po-210 source produces neutrons of about $1.5 \times 10^{3} \mathrm{n} / \mathrm{s}$. A PoBe source has an average neutron energy of $2.3 \mathrm{MeV}$. The calibration data for different detectors are shown in Appendix B.

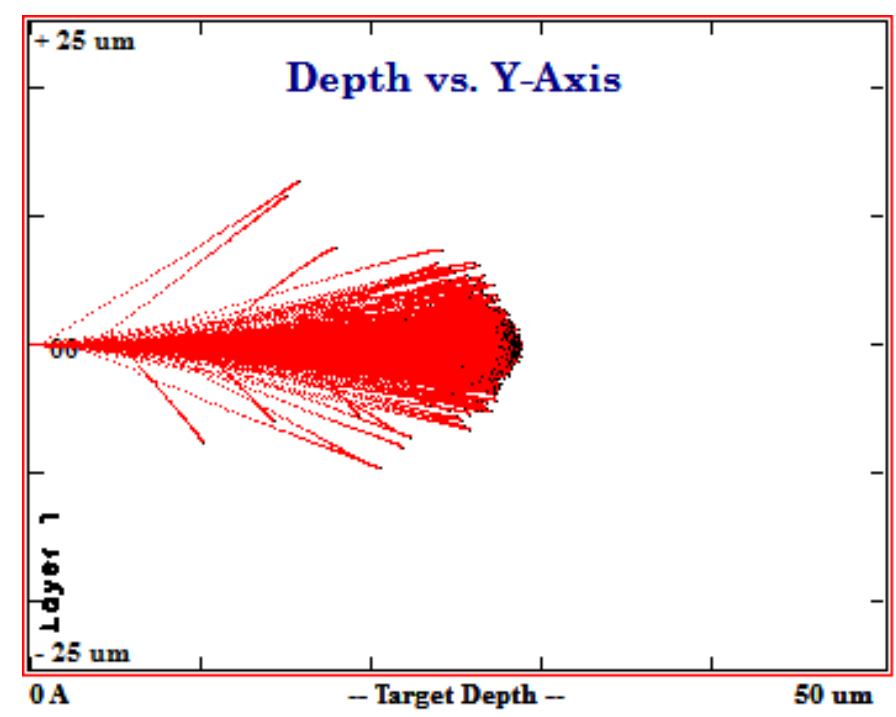

Figure 3-3: Range of 5.4 MeV ${ }^{4} \mathrm{He}$ Ion on Beryllium metal 
The calibration set-up is shown in Figure 3-4. The ${ }^{3} \mathrm{He}$ detector (Model LND 252257) used in this work was a cylindrical tube, $31.75 \mathrm{~cm}$ long with a radius of $1.397 \mathrm{~cm}$, enclosed in a thick steel wall to prevent electronic noise and to shield low energy gammas. The tube was filled with ${ }^{3} \mathrm{He}$ gas at pressure of 3040 torr. About $5 \%$ carbon dioxide is added to the gas to stabilize the counter. The detector was wrapped in a polyethene sleeve with height of $30 \mathrm{~cm}$ and a radius of $45 \mathrm{~cm}$. The detector was connected to an Ortec $142 \mathrm{PC}$ preamplifier, which was hooked to a Canberra Multichannel Analyzer (MCA) and Single Channel Analyzer (SCA) through an amplifier. The lower discrimination of the amplifier was set high enough to eliminate low-level noise and gamma ray, but still low enough to collect many of the low energy neutron events. A long period of background counts was collected before calibration of the detector to ensure that the average background counts were relatively low i.e. below 2 counts/100ms. The SCA was calibrated by matching the total counts recorded in the SCA with the total counts from the MCA.

The first step in the detector calibration was to determine the optimum operating voltage of the detector. The neutron detector operating voltage was obtained from the proportional region of the count rate against voltage curve. The operating high voltage was from chosen the proportional region, but well below the "plateau" region. The recommended voltage by the manufacturer for this detector is $950 \mathrm{~V}$. For this reason, the applied bias voltage of $950 \mathrm{~V}$ was used. The absolute efficiency of this detector has been previously measured by our group and determined to be $4 \%$.

The pulse height neutron spectrum at $950 \mathrm{~V}$ is show in Figure 3-5. Two peaks can be observed in the figure. The first peak occurring at $0.191 \mathrm{MeV}$ is the triton peak. The main peak corresponding to $765 \mathrm{keV}$ is a combination of both the proton and triton being absorbed in the detector gas. The low pulse height regions are due to noise from low energy electrons that are produced from gamma rays absorbed in the detector walls. 


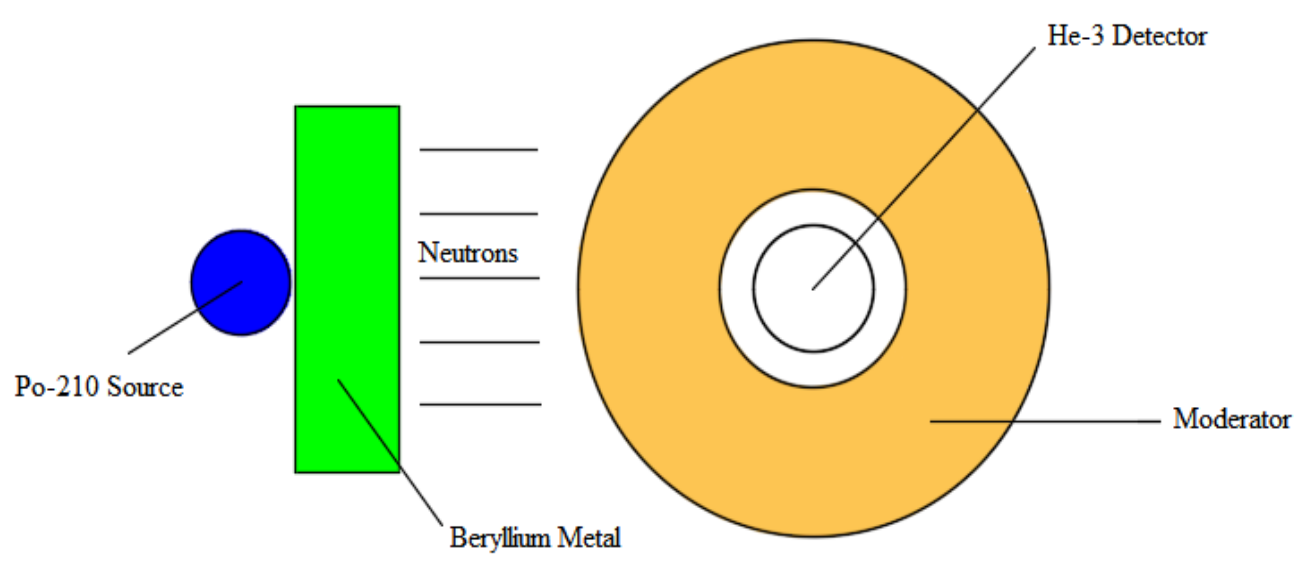

Figure 3-4: Set-up of He-3 detector Calibration

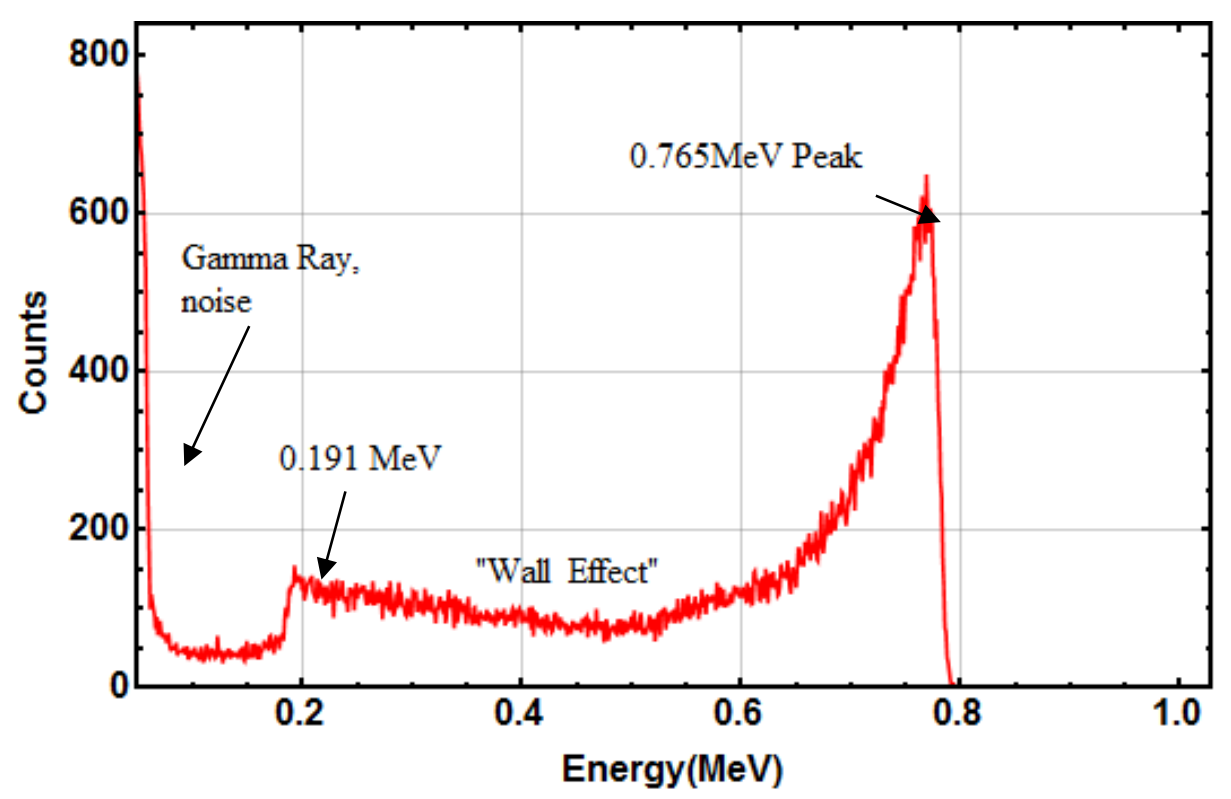

Figure 3-5: He-3 neutron pulse spectra

Proton recoil detector used in this work was obtained from LND, INC (Model 28129

Proton Recoil Fast Neutron Detector) [7]. The detector has a diameter of 38.1mm and length of $177.8 \mathrm{~mm}$ filled with hydrogen gas at a pressure of 3040 torr or 4 atms. The range of neutrons in hydrogen is given in this reference[8]. This detector is able to detect neutrons with energy less than $1.5 \mathrm{MeV}$. A small amount of carbon dioxide and nitrogen is usually 
added to the counter gas to improve the pulse rise time. The composition of nitrogen and carbon dioxide in these detectors is less than 5\%. Information about the proton recoil detector was obtained from LND Inc. The data sheet from the manufactural shows that the detector contains 38 torr of $\mathrm{CO}_{2}, 38$ torr of nitrogen and 2964 torr of hydrogen. Scattering from carbon and nitrogen does contribute substantially to the detector output. However, because the scattered neutrons can further undergo scattering with hydrogen before leaving the detector, it affects the pulse height spectrum indirectly. For carbon, between 0 to $28 \%$ of the initial neutron energy is lost as a result of scattering thereby leaving a subsequent recoil proton maximum energy of between 100 to $72 \%$ [9]. The cathode material of this detector is made from stainless steel. The detector can be operated at voltages between $2700-3000 \mathrm{~V}$, where $2850 \mathrm{~V}$ is the operating voltage recommended by the manufacturer. The raw pulse height spectra from the proton recoil detector are shown in figure 3-8. The detector was calibrated with a PuBe with source strength of about $5 \times 10^{6} \mathrm{n} / \mathrm{s}$. The source was placed 18.5 $\mathrm{cm}$ from the detector and 5400s counts were recorded. The theoretical spectrum of PuBe source is shown in Figure 3-6. The experimental set-up is shown in figure 3-7. PuBe source contains a mixture of $\mathrm{Pu}-239$ or $\mathrm{Pu}-238$ and Be-9. The half-life of $\mathrm{PuBe}$ is 24,114 years and it emits neutrons with average energy of between 4.2-5 MeV and maximum neutron energy of $11 \mathrm{MeV}$. No information has provided by the manufacture about the composition of plutonium and beryllium, the thickness of the steel wall and the area of vacuum between the source and the stainless steel. The absolute efficiency of this detector is about $0.0037 \%$. 


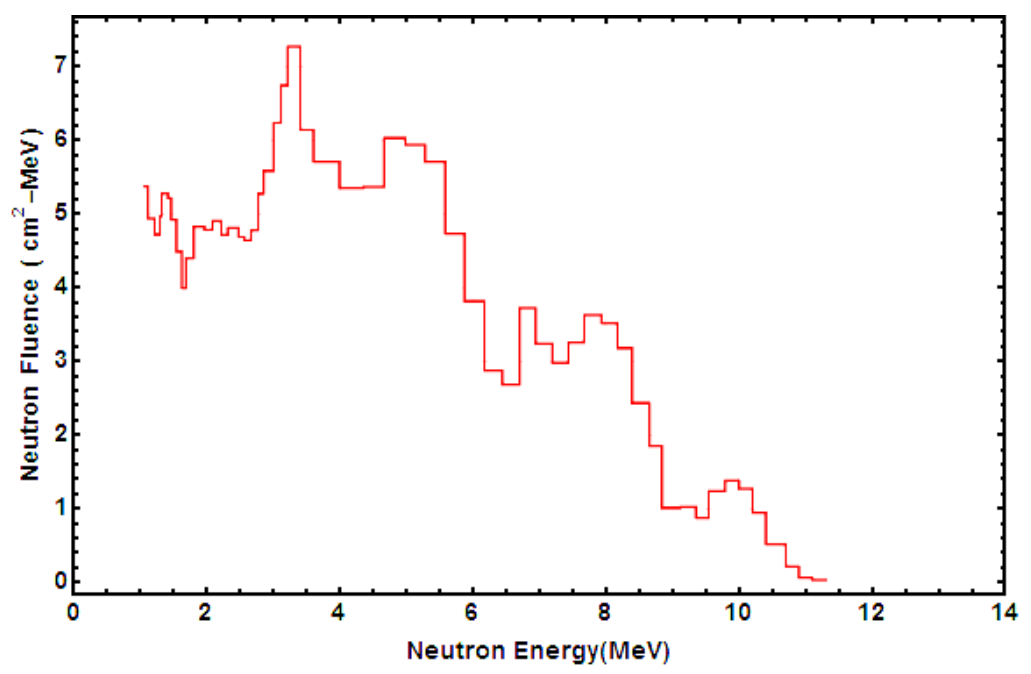

Figure 3-6: Theoretical Spectrum of PuBe [10]

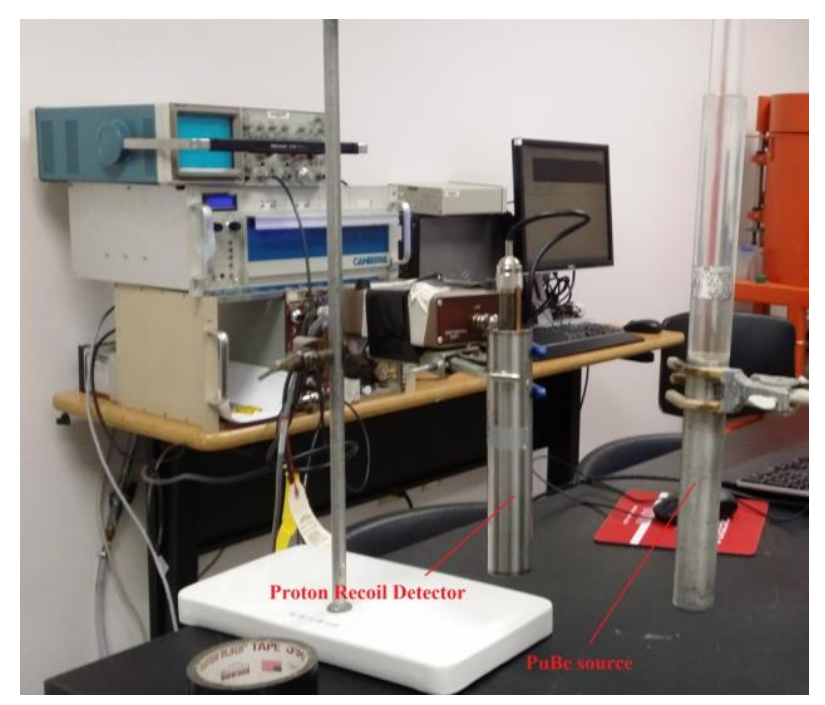

Figure 3-7: Set-Up of Proton Recoil Detector Calibration

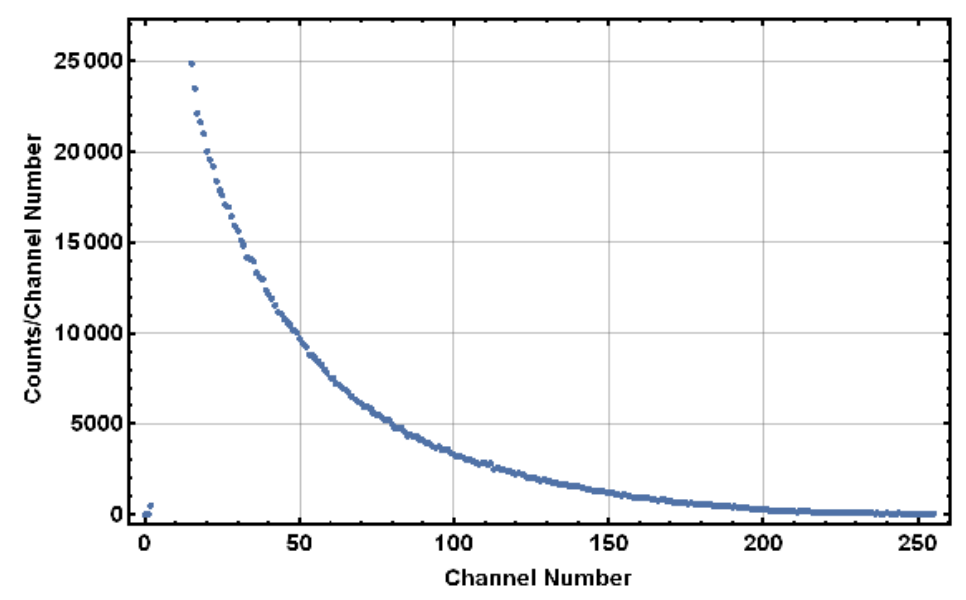

Figure 3-8: Raw Proton Recoil spectra of PuBe Source 


\subsection{X-ray Diffraction Measurements}

$\mathrm{X}$-ray diffraction was used to identify titanium deuteride in our experiment and to identify if any transmutation occurs during our investigation. X-ray diffraction method is an important technique to study crystal structures and atomic spacing. The importance of using $\mathrm{x}$-rays in instruments stems is that the wavelength of $\mathrm{x}$-rays is comparable to spacing of planes in crystal lattice. X-ray spectrometer is often used for performing non-destructive analysis of solids, powders and liquid specimens. Powder x-ray spectrometer has been widely used to identify unknown structures [10-12].

$\mathrm{X}$-ray diffractometer relies on constructive interface of monochromatic $\mathrm{x}$-rays and a crystal sample. An x-ray tube is used as a source of $x-y$ radiation. The production of $x$-rays is through the bombardment of a metal target in an evacuated x-ray tube with electrons. The operating voltage depends on the type of metal used. Most instruments used copper at the voltage at $40 \mathrm{kV}$ and current of $30 \mathrm{~mA}$. The x-ray passes through a series of closely spaced slits that collimate the beam. Constructive interface occurs when Bragg's law is satisfied.

$$
n \lambda=2 d \sin \theta
$$

Equation 9

Where $\theta$ the diffraction angle (Bragg's angle), $\mathrm{d}$ is the lattice spacing in a crystalline sample, $\lambda$ the wavelength of the x-ray and $n$ is a positive integer. After the beam is deflected by the specimens, it goes through another set of slits. These sets of slits reduced the background radiation, thereby improving the peak to noise ratio. This is important because it ensures that only scattered radiation from $\mathrm{x}$-ray interaction with the specimen is recorded. A position sensitive detector is used to detect the diffracted x-rays. The detector is easily displaceable along a measurement cycle for angular dependent measurement of scattered x-rays on the 
specimen. The goniometer is used for fine adjustment between the source and the detector.

The x-ray detector, the specimen and the x-ray source all lie in the circumference of a circle called the focusing circle. The angle between the x-ray source and the plane of the specimen is theta $(\theta)$, while the angle between the projection of the x-ray source and the detector is $2 \theta$. $\mathrm{X}$-ray pattern that used this geometry are known as $(\theta-2 \theta)$ scan. In such a configuration, the $\mathrm{x}$-ray source is fixed while the detector moves at different angle. The $2 \theta$ range usually varies from 0 to $170^{\circ}$ [13]. By scanning the sample in $\theta-2 \theta$ range, all possible diffraction pattern of the sample can be obtained. The material can be identified by determining the dspacing from the diffraction peaks since this parameter is unique for a given sample.

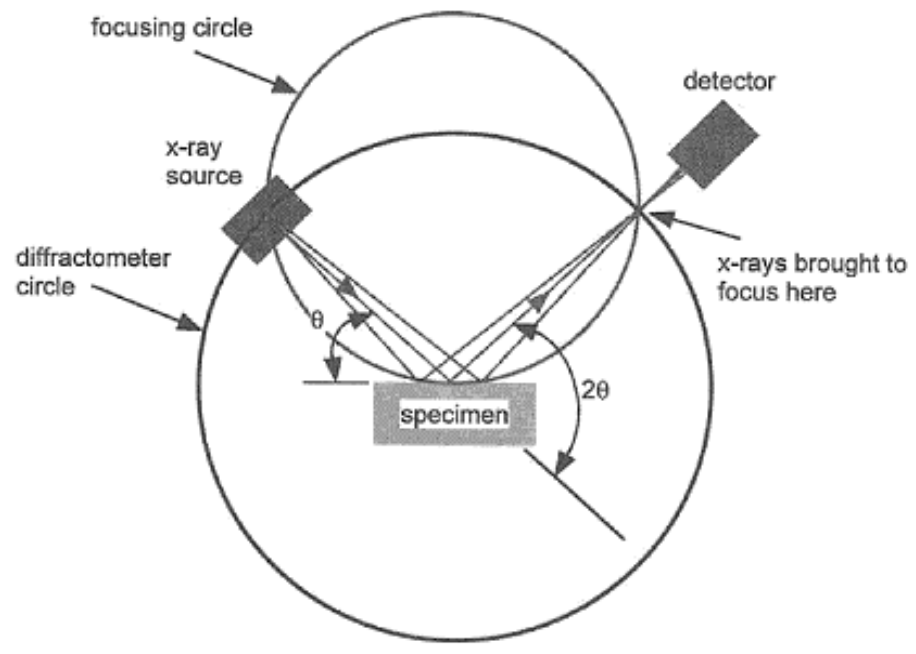

Figure 3-9: X-ray Diffraction Geometry [14]

X-ray diffraction (XRD) analysis was performed using a Rigaku powder diffractometer operating at a voltage of $40 \mathrm{kV}$ with a current of $44 \mathrm{~mA}$ using a $\mathrm{Cu} \mathrm{K} \alpha$ 
radiation. Diffraction patterns were measured in the $2 \theta$ range from $30-90^{\circ}$ with a step size of $0.005^{0}$

\subsection{Scanning Electron Microscope and Energy Dispersive Microscopy}

A Scanning Electron Microscope (SEM) is a powerful instrument to perform surface characterization of samples, providing high spatial resolution. The SEM produces high resolution of about 25 Armstrong. The scanning electron microscope consist of an electron gun that produces an electron beam, electromagnetic optics that guides and focuses the beam, and radiation detectors that record counts from scattered radiation from the sample. The electrons are generated by thermionic emission from a tungsten filament at $\sim 2700 \mathrm{~K}$. The electrons are accelerated towards the anode at a voltage between $200 \mathrm{~V}$ to $30 \mathrm{kV}$. The electron beam is incident on the area of the sample to be analyzed. The energy of the electron, the mass of the target and the atomic number of the target determines the type of interaction that occurs.

Secondary electrons, backscattered electrons, auger electrons, photons radiation and characteristics X-rays of various energies are produced. Secondary electrons are produced because of the inelastic collision and scattering of incident electrons with the specimen and can be used to reveal surface structures of materials with resolution of about $10 \mathrm{~nm}$.

Backscatter results from elastic collision and scattering between the nuclei of the specimen and the incoming electrons. Backscatter electrons are generated deeper within a specimen and can reveal the atomic number contrast and topographical contrast with resolution of more than 1 micron. X-ray signal can penetrate deeper in the sample and be use for determination of elemental composition of the specimen using a technique known as energy dispersive spectroscopy [14-17]. The Hitachi S-4700 Field Emission Scanning Electron Microscope was used for analyzing the titanium sample. 


\subsection{Liquid Scintillation Detector}

A liquid scintillator counter was used to determine the presence of tritium from titanium hydride. Tritium is a nuclear product that is expected from the titanium-deuterium reaction. Some experiments have confirmed tritium production. The LSC technique has been widely used for detection and quantification of nuclear emissions (alpha, and beta emitting radionuclide). Liquid scintillation counters generally have high detection efficiency because of the proximity of the sample to the detector. Tritium is a radioactive isotope of hydrogen with an excess of a neutron in its nucleus. It decays to a beta emission releasing and neutrino in the process. The decay energy of $18.6 \mathrm{keV}$ is emitted in the process, which is shared between the antineutrino and the beta particle. The beta particle has energy between 0 and $18.6 \mathrm{KeV}$. The beta spectrum of tritium is shown in the diagram below:

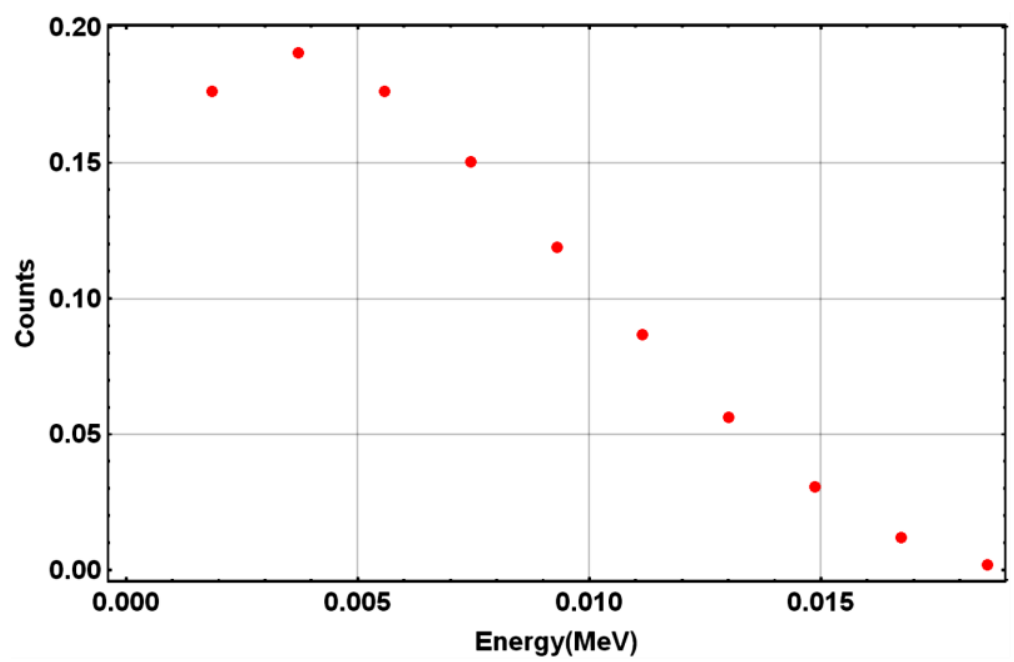

Figure 3-10: Theoretical Spectrum of Tritium [19]

The liquid scintillation detection method is based on the detection of light by photomultiplier tubes due to excitation of molecules of a solution resulting from the absorption of beta radiation. The scintillation solution (cocktail) is a mixture of a solvent (used to ensure efficient transfer of energy) and a solute (the solute is the scintillator). The collision between a beta particle and the solvent leads to excitation of molecules of the solvent. The solvent can transfer its energy from one molecule to another or to the solute resulting in scintillation. About 
10 photons per keV are produced in a decay event. The quantum efficiency (conversion efficiency from a photon to a photo-electron) of this process is only about $30 \%$. Therefore for low energy beta radiation such as tritium with an average beta energy of $6 \mathrm{keV}$, all the radiation cannot be detected at $100 \%$ efficiency as many events do not produce a sufficient number of photons[18].

The scintillator converts the kinetic energy of the beta into electrical energy. The scintillation intensity ranges from 0 to $18.6 \mathrm{keV}$. The relative intensity depends on the type of liquid scintillation cocktail, the radiolabeled analyte, other solutes dissolved along with the analyte, sample vial material, quantity of radiolabeled analyte, maximum energy of emission, and chemically processes involved affecting radiochemical distribution [19]. The reaction between the beta radiation and the scintillation material leads to the emission of light in the ultra violet region of the electromagnetic spectrum. A photomultiplier is used to amplifier the light output and to convert the signal to a form that is easier to detect. The PMT is a linear device; hence the amplitude of the pulse is directly proportional to the number of photons detected by the photocathode. The scintillation is detected and converted into an electrical pulse. The number of pulses registered is a measure of the number of scintillation events that occurs $[20,21]$. The signal from a PMT is fed into a circuit that produces an output only when both PMT received a signal within the resolving time of the circuit. This method ensures that the signal outputs are counted when they reach both photomultiplier tubes while spurious pulses, which results from noise and is detected by only one of the PMT is not counted. This process is called time coincidence counting. 


\section{REFERENCES}

1. collaboration, G., GEANT4 10.0 Release Notes,(2013).

2. $\quad$ P-2042-1000 Staticmaster ${ }^{\circledR}$ Nuclespot ${ }^{\mathrm{TM}}$ Alpha Ionizer $\mid$ NRD Static Control LLC.

3. Lehman, R., The origin of neutron groups in $B e(\alpha, n)$ sources. Nuclear Instruments and Methods, 1968. 60(3): p. 253-260.

4. Ziegler, J.F., M.D. Ziegler, and J.P. Biersack, Srim. 2008: Cadence Design Systems.

5. $\quad$ Ensslh, N., The Origin of Neutron Radiation.

6. Halpern, I., The $(\alpha, n)$ Cross Sections of Beryllium, Magnesium, and Aluminum. Physical Review, 1949. 76(2): p. 248.

7. $\quad$ Eckerman, K., et al., Availability of nuclear decay data in electronic form, including beta spectra not previously published. Health physics, 1994. 67(4): p. 338-345.

8. Werle, H., Spectrum measurements of radioactive neutron sources in the $10 \mathrm{keV}$ to 10 $\mathrm{MeV}$ energy region with proton-recoil proportional counters(Proton recoil proportional counters for spectrum measurements of radioactive neutron sources of Ra-Be, Am-B, Am-F, Am-Li, and Cf-252). 1971.

9. Knoll, G.F., Radiation detection and measurement. 2010: John Wiley \& Sons.

10. Segal, L., et al., An empirical method for estimating the degree of crystallinity of native cellulose using the X-ray diffractometer. Textile Research Journal, 1959. 29(10): p. 786-794.

11. Spurr, R.A. and H. Myers, Quantitative analysis of anatase-rutile mixtures with an Xray diffractometer. Anal. Chem, 1957. 29(5): p. 760-762.

12. Moore, D.M. and R.C. Reynolds, X-ray Diffraction and the Identification and Analysis of Clay Minerals. Vol. 378. 1989: Oxford university press Oxford.

13. Suryanarayana, C. and M.G. Norton, X-ray diffraction: a practical approach. 2013: Springer Science \& Business Media.

14. Goldstein, J., et al., Scanning electron microscopy and X-ray microanalysis: a text for biologists, materials scientists, and geologists. 2012: Springer Science \& Business Media.

15. Todokoro, H. and M. Ezumi, Scanning electron microscope. 1999, Google Patents.

16. Joy, D.C., Scanning electron microscopy. 2006: Wiley Online Library.

17. Unakar, N., J. Tsui, and C. Harding, Scanning electron microscopy. Ophthalmic Research, 1981. 13(1): p. 20-35.

18. Kessler, M.J., Liquid Scintillation Analysis: science and technology. 1989: Packard Instrument.

19. Kessler, M.J., LIQUID.

20. Peng, C., Liquid scintillation counting. CG Bell, Jr. and FN Hayes, eds, 1958: p. 198.

21. Nurmi, J., K. Rundt, and T. Oikari, Liquid scintillation counter. 1987, Google Patents. 


\section{RESULTS AND DISCUSSION}

Several experiments were conducted throughout this work. The experimental method was modified based on initial results. For example, in some of the runs, only proton recoil detectors and moderated detectors were used, while in other experiments, proton recoil detectors, moderated helium-3 detectors, and unmoderated helium-3 detectors were used. The method used in achieving thermal shock in the experimental chamber was also modified - in some cases, a heat gun was used instead of boiling water to achieve the thermal shocking procedure. The motivation of using a heat gun was to ensure a non-equilibrium temperature distribution in the experimental system and to achieve a wider temperature variation. The non-equilibrium condition and or change in thermodynamic condition in the titanium-deuterium system is thought to be responsible for nuclear emission. After the first three experiments, a batch script was written to store spectra data from the Genie 2000 software every 60s, for the moderated and unmoderated helium-3 detectors, and every 600s seconds for the proton recoil detector (because a sufficient time is needed to obtain a neutron spectrum with a proton recoil detector). The data from the moderated and unmoderated helium detectors were later processed and the count rates were compared with counts rates from the SCA- obtained using the Labview program.

This section discusses the results of nine experiments conducted to investigate neutron emissions from titanium-deuterium systems under thermal shock. The first results are those pertaining to the case where thermal shocking was achieved by using boiling water and the second section is for the case of shocking using a heat gun.

\subsubsection{Shocking through boiling water}

These sets of experiments involved loading titanium in our thermal shock chamber, and later transferring the chamber into liquid nitrogen to achieve maximum loading, followed 
by rapid thermal cycling in boiling water. The samples used in these experiments were dehydrided titanium powder, -325 mesh with 99.99\% purity purchased from Alfa Aesar. Several long background counts were collected prior to running the experiments. The background counts were used for uncertainty quantification with experimental results. The experimental chamber was loaded with titanium powder and deuterium gas ( $99.96 \%$ purity) was introduced into the chamber at a pressure of about 200 psig at room temperature. Prior to loading, the system was baked at high temperature $\left(\sim 300^{\circ} \mathrm{C}\right)$ using a heat tape to remove any contamination in the system. The chamber was leak-tested with a helium-3 mass spectrometer. The chamber was later placed in liquid nitrogen bath for six hours to ensure adequate deuterium loading. The counting system was started at this point to record the pressure, temperature, and the neutron count rate. A drop in the pressure of experimental chamber is usually observed, which is, in part, due to a decrease in temperature of the experimental chamber, and because of the formation titanium deuteride (the chamber pressure after the system is placed in liquid nitrogen is not constant because only part of the system is placed in liquid nitrogen). The formation of titanium deuteride is exothermic; hence there will be a temperature increase in the titanium sample which is monitored using a thermocouple placed inside the chamber. The experimental chamber was then rapidly transferred into boiling water bath at $100^{\circ} \mathrm{C}$.

\section{EXPERIMENT 1}

The experimental chamber in experiment 1 was loaded with $8.55 \mathrm{~g}$ of titanium powder in a glove box under an argon atmosphere. Just before loading the sample in the chamber, the composition of this powder was determined by Energy Dispersive Spectroscopy (EDS). Two areas were selected within the sample for this analysis. The details of the regions and the composition of the titanium are in Appendix C and Table 4-1, respectively. The data sheet obtained from the manufacturer indicates that this titanium contains about $99.9 \%$ titanium 
and $0.01 \%$ impurities consisting of the following elements

( $\mathrm{Li}(<0.03), \mathrm{Mg}(<0.54), \mathrm{S}(<4.7), \mathrm{Mn}(0.083), \mathrm{Cu}(0.24), \mathrm{As}(<0.1), \mathrm{Mo}(0.10), \mathrm{Sn}(<0.3), \mathrm{B}(<0.03), \mathrm{Al}($

1.6), $\mathrm{Cl}(0.31), \mathrm{Ru}(0.44), \mathrm{Fe}(10), \mathrm{Zn}(<0.1), \mathrm{Se}(<0.3), \mathrm{Ag}(0.3), \mathrm{Sb}(<0.3), \mathrm{F}(<0.3), \mathrm{Si}(1.8), \mathrm{K}(0.40), \mathrm{V}($

0.12), $\mathrm{Co}(0.032), \mathrm{Th}(<0.005), \mathrm{Pb}(<0.05), \mathrm{Zr}(0.10), \mathrm{Cd}(<0.3), \mathrm{Te}(<0.3), \mathrm{Na}(0.13), \mathrm{P}(0.071), \mathrm{Ca}(0.62$

)$, \mathrm{Cr}(1.2), \mathrm{Ni}(0.76), \mathrm{U}(<0.005), \mathrm{Rb}(<1), \mathrm{Nb}(<0.05), \mathrm{In}(<0.1), \mathrm{W}(0.35))$. No carbon is listed as impurities, but the sample analysis was performed by placing the titanium powder on a carbon tape. This is likely reason why carbon is found in the data in Table 4-1.

Table 4-1: Composition of Titanium in Experiment 1

\begin{tabular}{|c|c|c|c|c|}
\hline $\begin{array}{c}\text { Elemental } \\
\text { Comp. }\end{array}$ & Atomic \# & Mass [\%] & $\begin{array}{c}\text { Mass } \\
\text { Norm. [\%] }\end{array}$ & $\begin{array}{c}\text { Abs. error } \\
{[\%], 1 \sigma}\end{array}$ \\
\hline Titanium & 22 & 103.20 & 98.87 & 3.56 \\
\hline Carbon & 6 & 1.18 & 1.13 & 0.20 \\
\hline
\end{tabular}

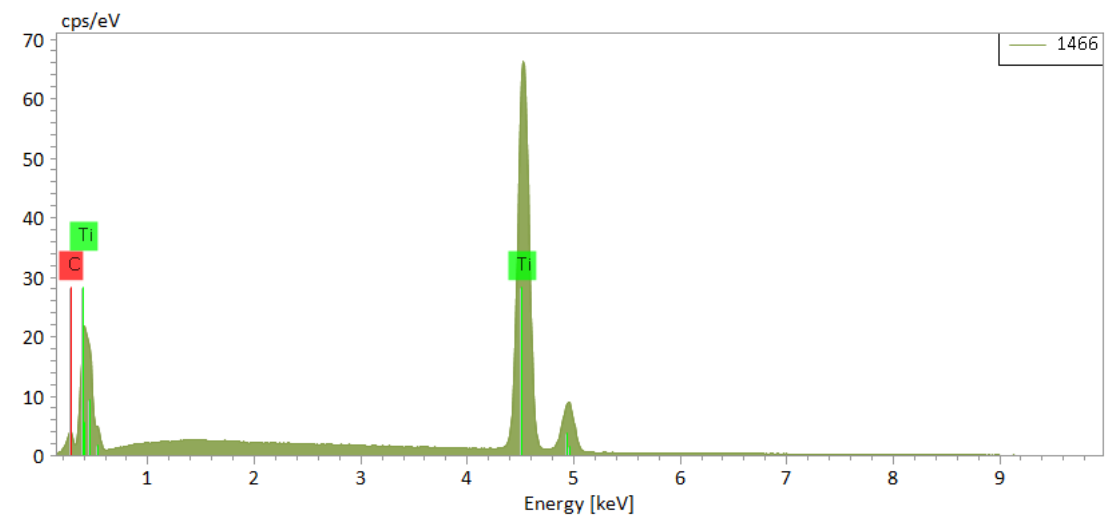

Figure 4-1: EDS spectra of titanium powder used in Experiment 1

The background count rate of experiment 1 is shown in figure 4-2. The counts were collected every $0.1 \mathrm{~ms}$ using the LabVIEW data analysis program. The count rate represents a time stamp of every neutron produced during measurement. The counts were collected for a period of four days with the program restarted every 24 hours. The long background counts reduce the uncertainty in the measurement. A moderated helium-3 and a proton recoil detector were 
used in to measure count rates. The maximum count rate of 2 was recorded with the moderated helium-3 detector. Meanwhile, the proton recoil detector recorded a maximum count of 2 and an average count of $0.53 \mathrm{c} / \mathrm{mins}$. The background count rate for moderated helium-3 detector and the proton recoil detector are shown in the figure 4-2 and figure 4-3, respectively.

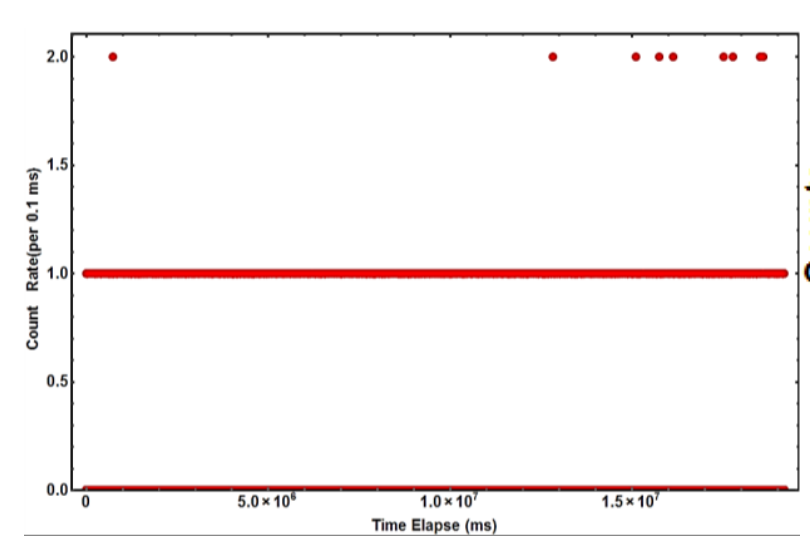

Figure 4-2: Moderated He Detector-Background Count

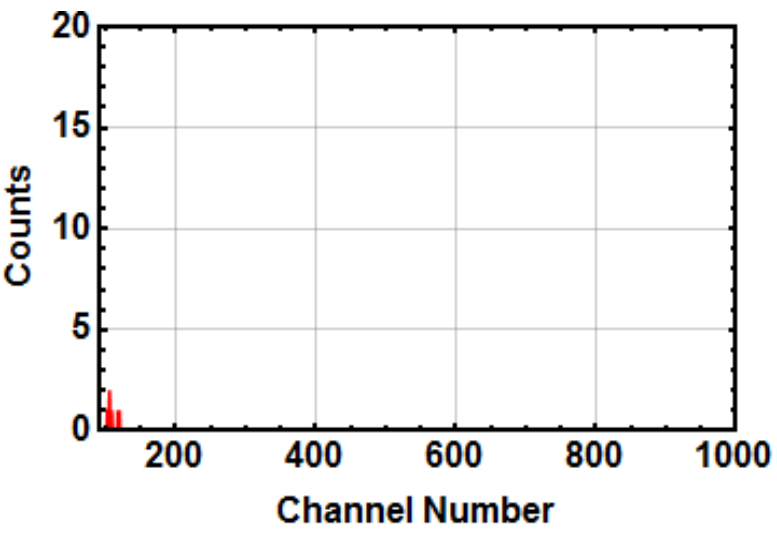

Figure 4-3: -Proton Recoil- Background Count

The results from the thermal shock experiment is shown in figure 4-4 to figure 4-8. The first half of the graph shows the region where the system was in liquid nitrogen (temperature of $\left.\sim 192^{\circ} \mathrm{C}\right)$. A "K" thermocouple was used in this measurement. These thermocouples do not operate well at liquid nitrogen temperatures. The second part of the graph shows a region where there is a huge increase in temperature, representing the region where the thermal shock system was transferred into boiling water. Because of the impurities in water, the temperature values $\left(\sim 95^{\circ} \mathrm{C}\right)$ is lower than boiling water temperature $\left(\sim 100^{\circ} \mathrm{C}\right)$. 


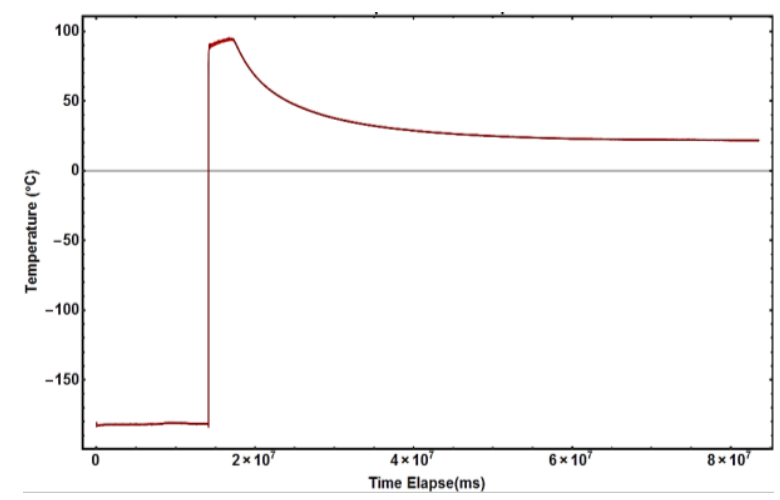

Figure 4-4: Inside Temperature vs time

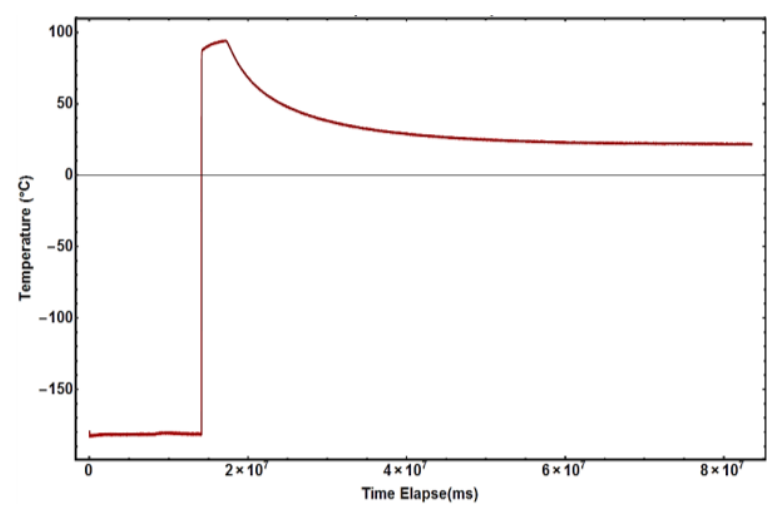

Figure 4-5: Outside Temperature vs time

Figure 4-6 shows the pressure variation over time, during the experiment. The pressure and temperature are directly related, i.e. when the system is moved into liquid nitrogen, the pressure decreases, and the pressure increases when the system is transferred into hot water. It is this rapid pressure change that forces deuterium atoms in the titanium lattice to interact with each other leading to neutron emissions. It is at this point that neutron bursts are expected in the experiment. As seen in figure 4-7 and figure 4-8, no neutron count rate greater than the maximum background neutron count rate was observed in the helium-3 and the proton recoil detector throughout the experiment. Figure 4-8 is the counts recorded in the proton recoil detector for a period of ten minutes during shocking.

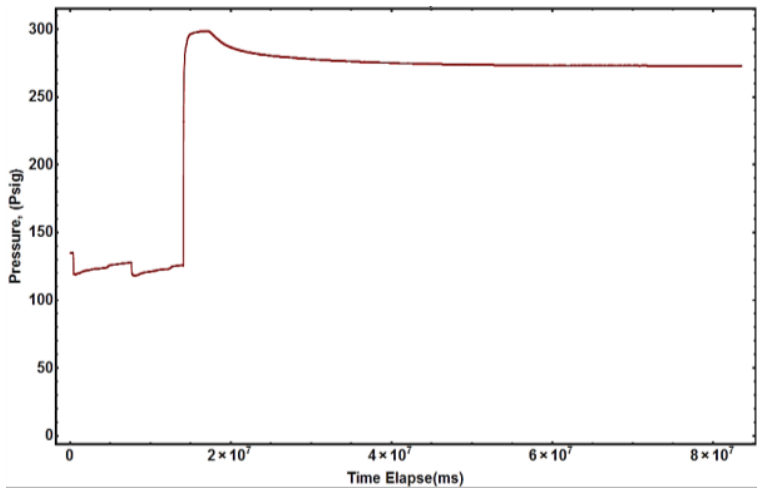

Figure 4-6: Pressure Variation over time

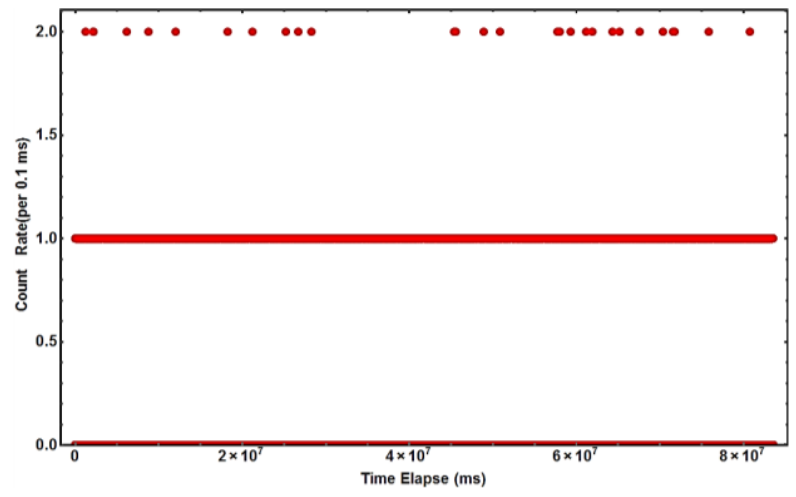

Figure 4-7: Moderated He-3- Foreground Count Rate 


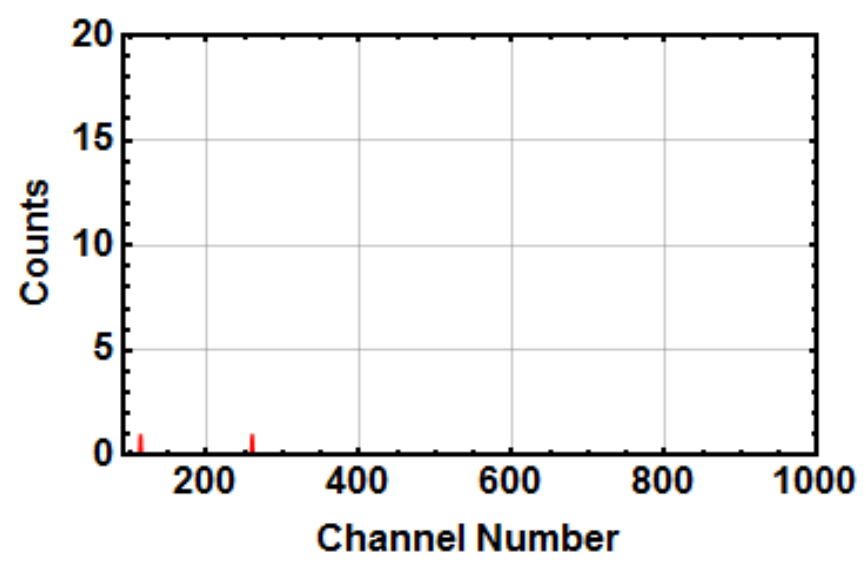

Figure 4-8: Foreground Count in Proton Recoil Detector

After the experiment, the titanium powder was removed from the experimental chamber under an argon atmosphere in an air-tight glove box. The surface of the sample was analyzed using a Scanning Electron Microscope (SEM) to study the morphology of the sample and to determine if any new element was produced. No morphological change was observed on the surface of the titanium powder. The images of the showing the morphology of the sample after the experiment is shown in figure 4-9 and figure 4-10.

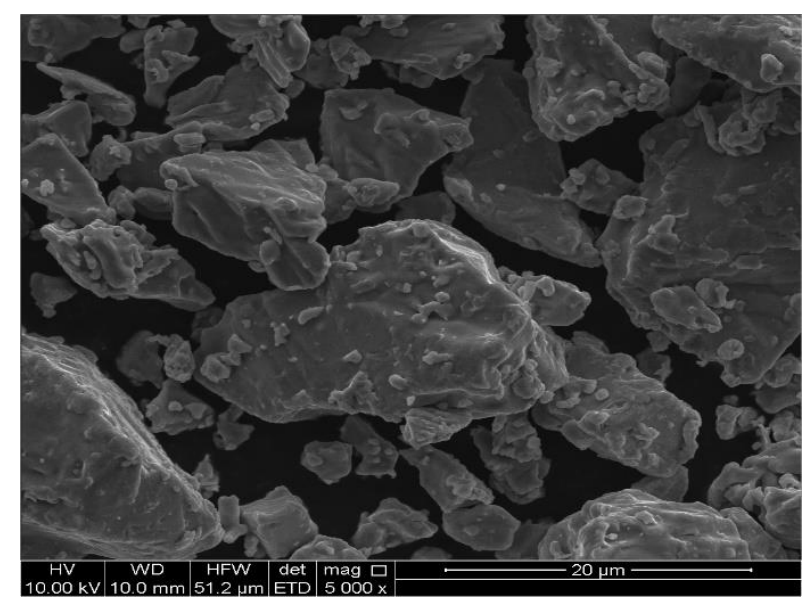

Figure 4-9: Titanium Powder-Control Sample

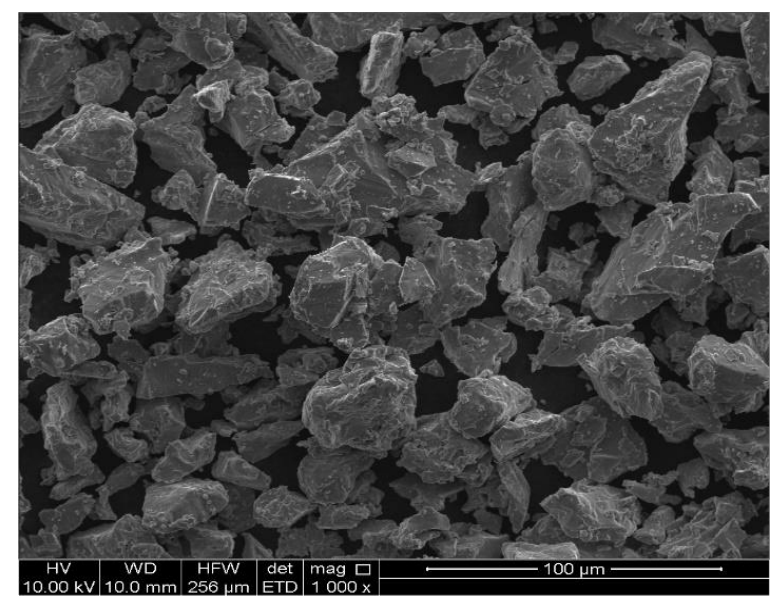

Figure 4-10: Titanium powder - Experimental Sample 


\section{EXPERIMENT 2:}

Table 4-2 shows the composition of the titanium sample used in this experiment. Energy dispersive analysis of the control sample shows that it contains $97.21 \%$ mass of titanium and $2.17 \%$ mass of carbon. The composition of the titanium powder was determined using the EDS technique. The results are given below:

Table 4-2: Composition of Titanium

\begin{tabular}{|c|c|c|}
\hline $\begin{array}{c}\text { Elemental } \\
\text { Comp. }\end{array}$ & $\begin{array}{c}\text { Atomic } \\
\#\end{array}$ & $\begin{array}{c}\text { Mass } \\
\text { Norm. [\%] }\end{array}$ \\
\hline Titanium & 22 & 97.21 \\
\hline Carbon & 6 & 2.79 \\
\hline
\end{tabular}

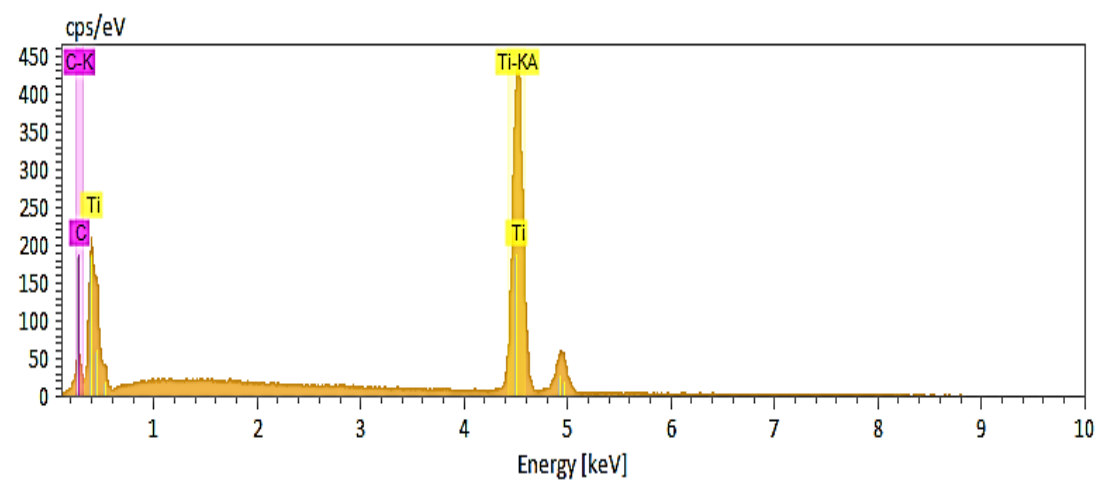

Figure 4-11: EDS spectra of Titanium

The carbon in the Table 4-2 resulted from of the carbon tape used during the EDS analysis. The background count rate (counts/0.01ms) is shown in the figure 14-12. A moderated, an unmoderated helium-3, and a proton recoil detector were used to monitor this experiment. The maximum recorded count rate (counts $/ 0.01 \mathrm{~ms}$ ) of 2 was recorded in both the moderated and unmoderated helium-3 detectors. No counts were observed in the proton recoil detector. The thermal shock chamber was loaded with $15.42 \mathrm{~g}$ of titanium powder. The variations in temperature and pressure over time during the experiment is shown in figure 14.14 , figure 14.15, and figure 14.16. The pressure data decreases in steps of 20 because LabVIEW program recorded the data only to one decimal places and the conversion of voltage measured to pressure yielded a significant error. The number of decimal places was increased in later 
experiments to reduce the error. After loading the system with deuterium, it was observed that the pressure in the system began to drop very fast, indicating that the system was absorbing deuterium.
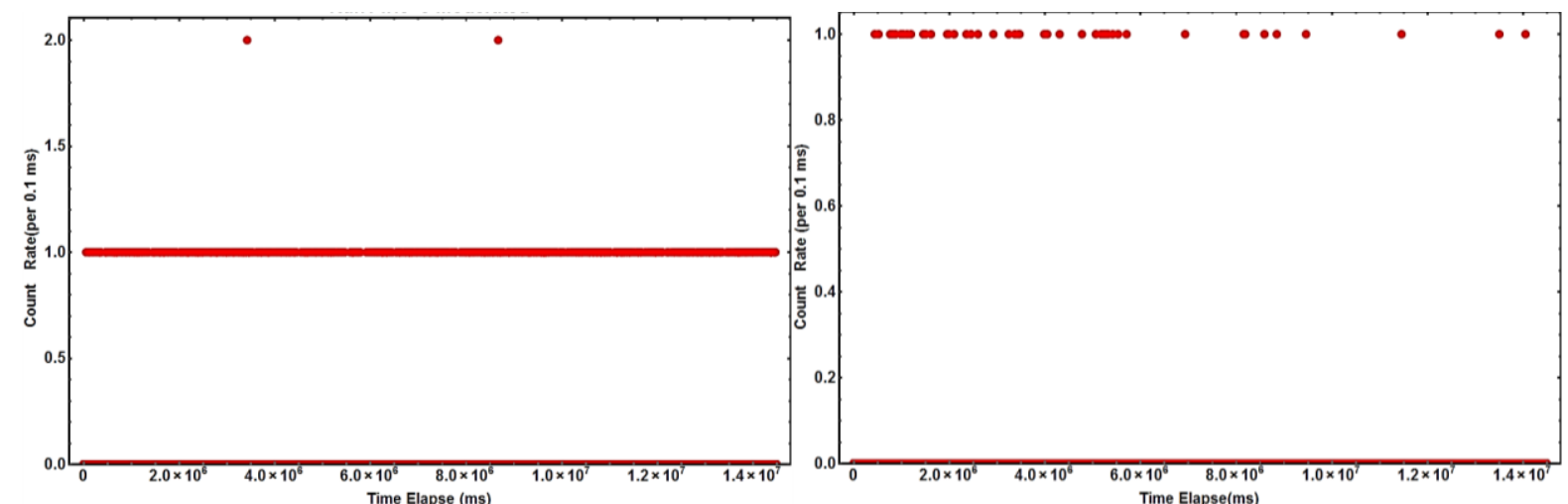

Figure 4-12: moderated ${ }^{3} \mathrm{He}$-Background Count Rate Figure 4-13: Unmoderated ${ }^{3} \mathrm{He}$ Background Count Rate

The system was later transferred into liquid nitrogen. After about 4 hours in liquid nitrogen, it was observed that all the deuterium had been absorbed into the titanium lattice. The system was then transferred into boiling water to force some of the deuterium out of the titanium lattice, so as to induce a titanium-deuterium reaction. However, no pressure change was observed in the experimental system. The reason why no pressure change was observed is that the deuterium atoms are tightly bound to the titanium atoms such that the applied temperature was not sufficient to force the deuterium out of the titanium lattice. The conclusion was to increase the amount of deuterium into titanium lattice and allow for the system to reach equilibrium (where the titanium cannot absorb any more deuterium) before shocking the system. This approach was employed in subsequent experiment.

The foreground count rate during this period is shown is figure 17-18. The count rate from the moderated detector is due to electronic noise while moving the thermal shock chamber. After the first trial (shocking the system), the system was removed from boiling water and more deuterium was added. Counts were recorded during this period. 


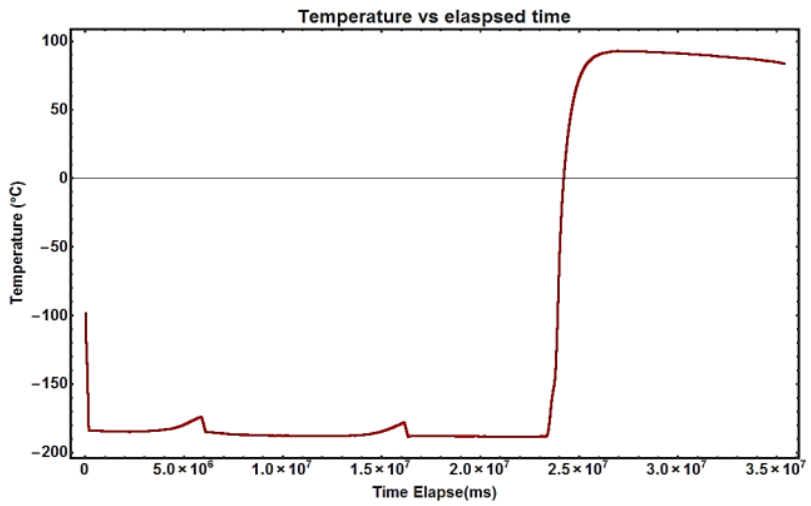

Figure 4-14: Inside Temperature vs time

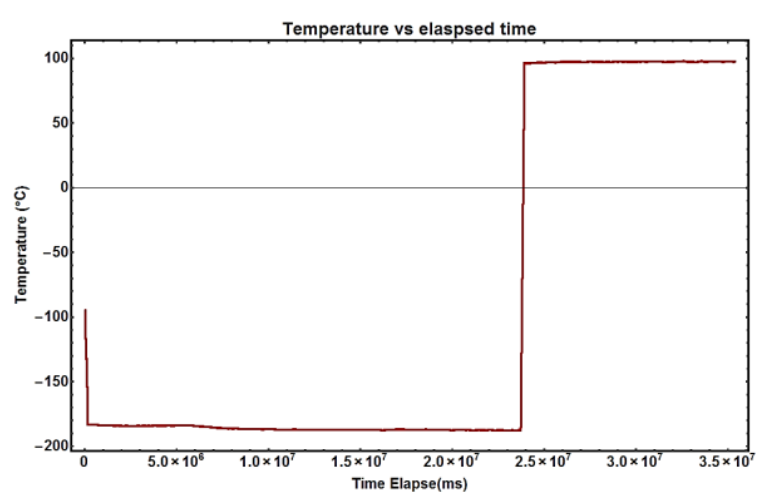

Figure 4-15: Outside Temperature vs time

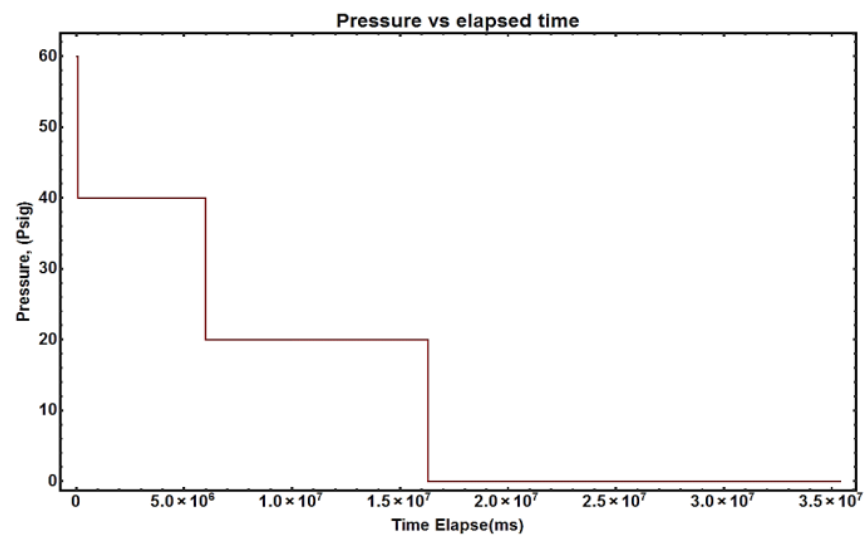

Figure 4-16: Pressure variation with time
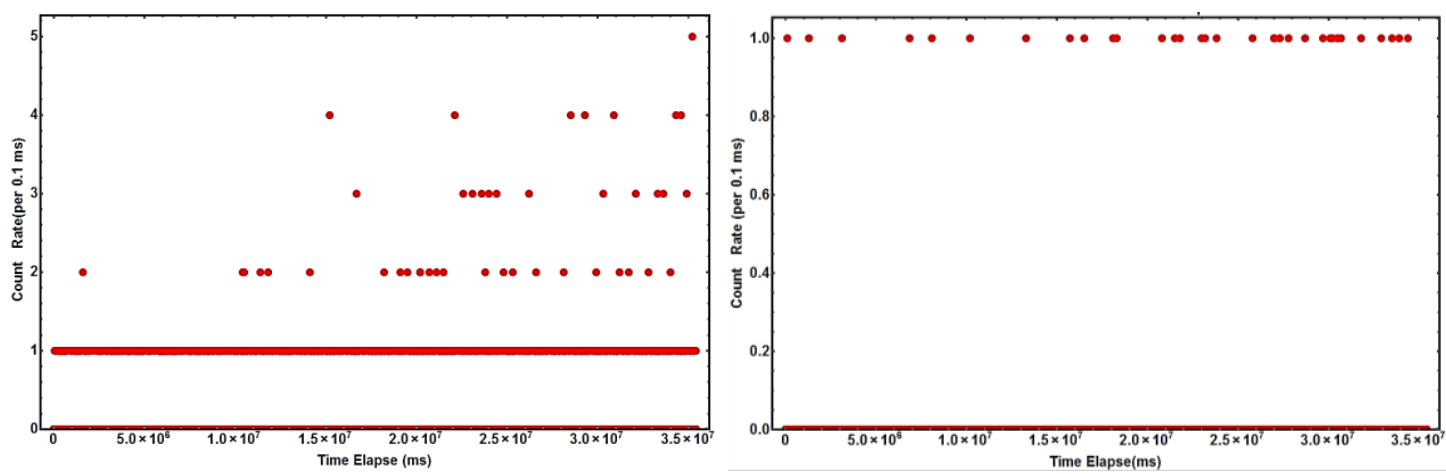

Figure 4-17: Moderated ${ }^{3} \mathrm{He}$-Foreground Count Rate $\quad$ Figure 4-18: Unmoderated ${ }^{3} \mathrm{He}-3$-Foreground Count Rate

After loading more deuterium in thermal shock chamber, on the fourth day (after which the system has been loaded with a total pressure of $455 \mathrm{psig}$ ), a huge temperature increase was observed. The increased in temperature produced an observable change in the color of 
stainless steel. Figure 14-19 shows the region in the thermal shock system where the color changed to brown.

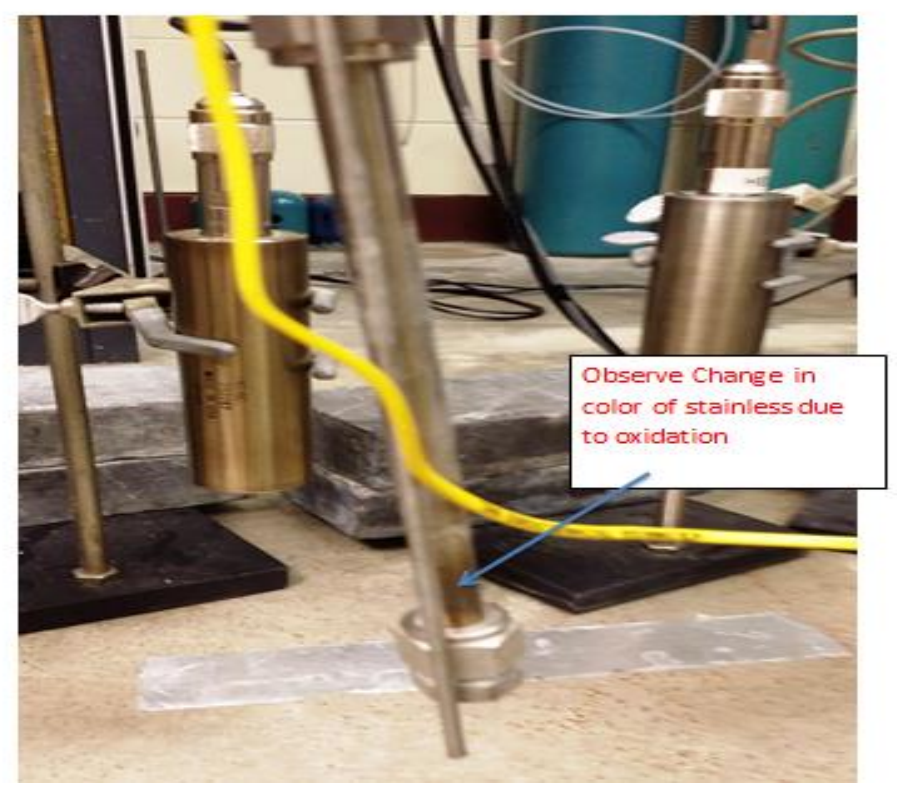

Figure 4-19: Oxidation observed on Stainless Steel

Stainless steel will only change its colour to brown above $400{ }^{\circ} \mathrm{C}$. The graph below shows that the observed phenomena occurred at much higher temperature $\left(420^{\circ} \mathrm{C}\right)$ (There was a lot of noise in thermocouple data due to poor data sampling). The huge temperature increase was followed with a rapid absorption of deuterium. After the system return to room temperature and after loading more deuterium, on the fifth day, the system was again placed in liquid nitrogen, and later it was transferred into boiling water to complete the thermal shock procedure. No neutron burst was observed. 


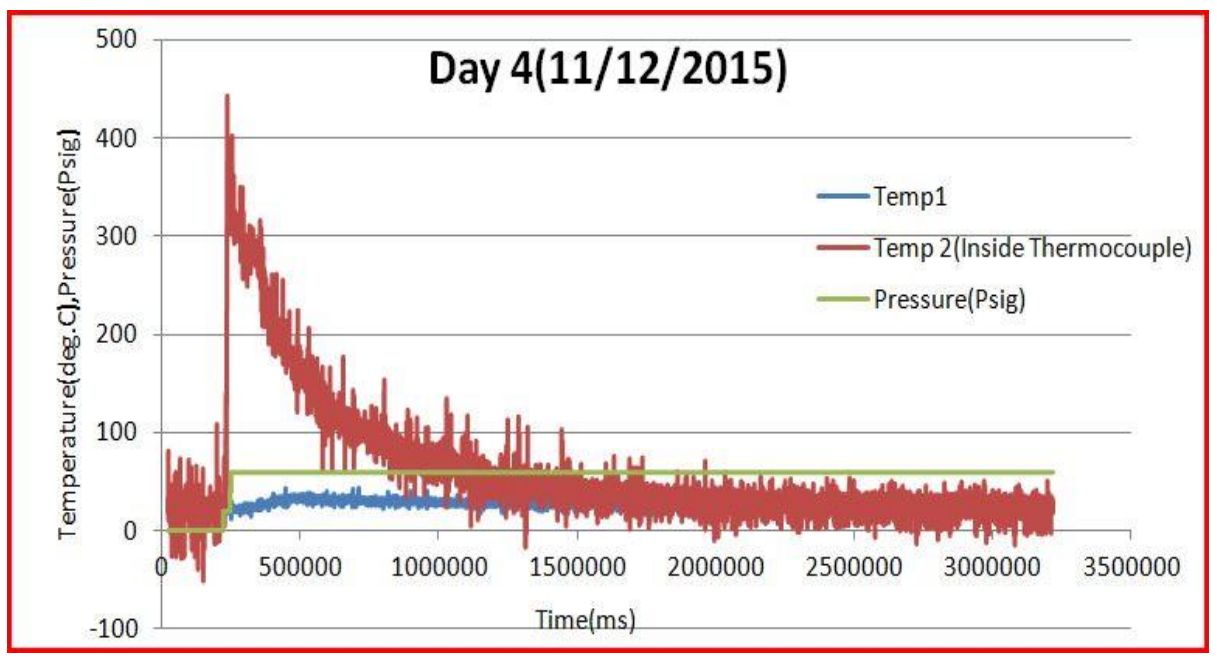

Figure 4-20 : Huge Temperature Observed in the titanium - deuterium System

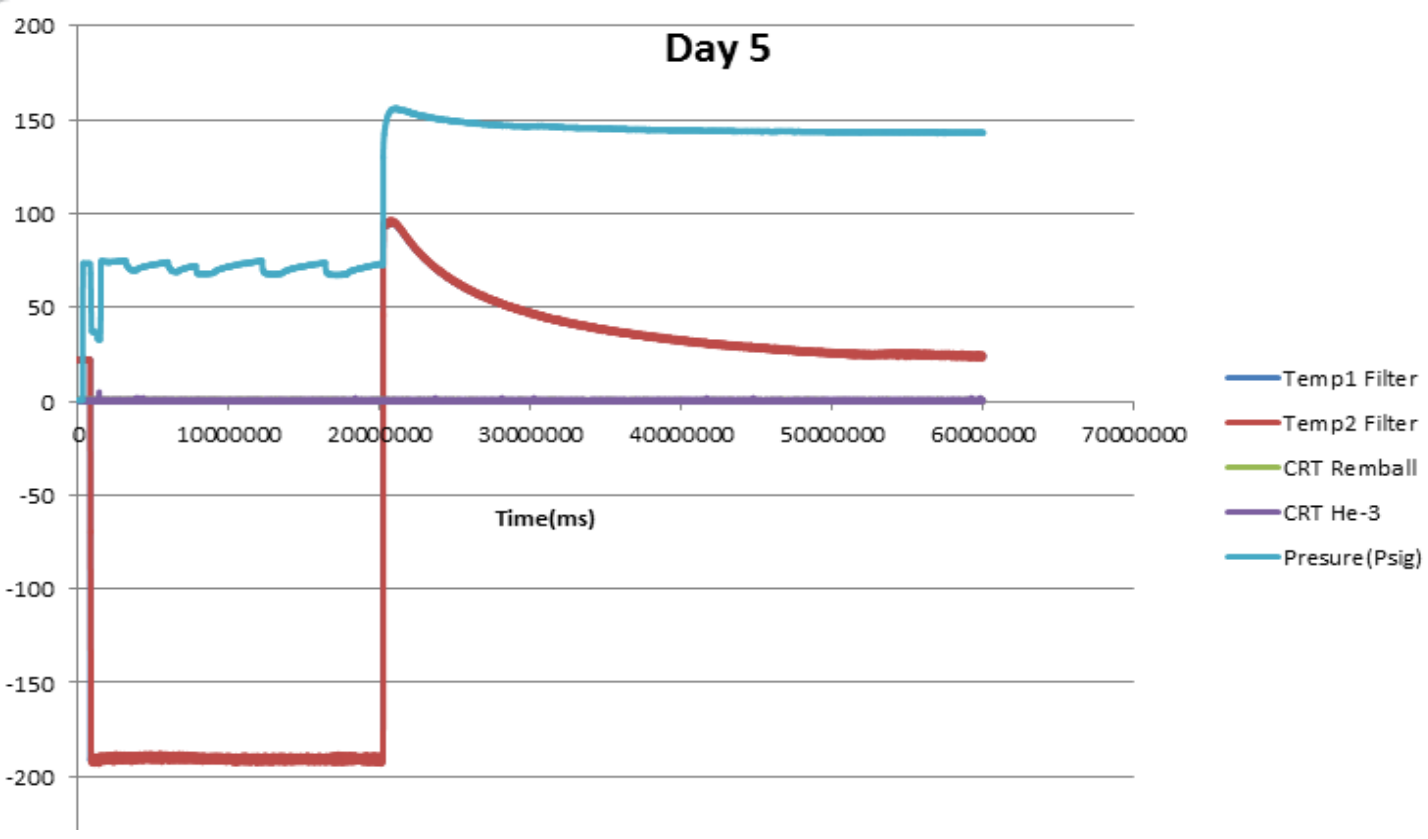

Figure 4-21: Experiment 2- Day 5 Results

After the experiment, the titanium powder was removed from the thermal shock chamber under an argon atmosphere and the sample was analyzed with a SEM and an X-ray diffractometer. Figure 22-23 shows the SEM images of control and experimental samples. The scanning electron microscope images show cracks produced in the experimental sample during deuterium absorption which were not present before deuterium loading. Cracks are 
known to be formed when metal forms hydrides. According to the fracto-fusion mechanism cracks produced within a material can generate sufficient electric field or temperature gradient within a crack initiating a local nuclear reaction. Neutrons are hypothesized to be produced because of this process [7-10]. The number of cracks formed depends on the level of deuterium absorption. Since the cracks occurred at different locations throughout the sample, the nuclear reaction should it occur should take place in these locations. Hence, the level of neutron produced should depend on the number of cracks. The cracks also occurred at random locations within the sample and it is difficult to determine where these cracks would be formed. This nuclear process, should it occur should be a random process in condense matter. However, no neutron was observed in this experiment, thus indicating that the crack formed in titanium samples may not be necessary for the neutron production, but other conditions are needed to achieve the titanium-deuterium reaction leading to neutron emission.

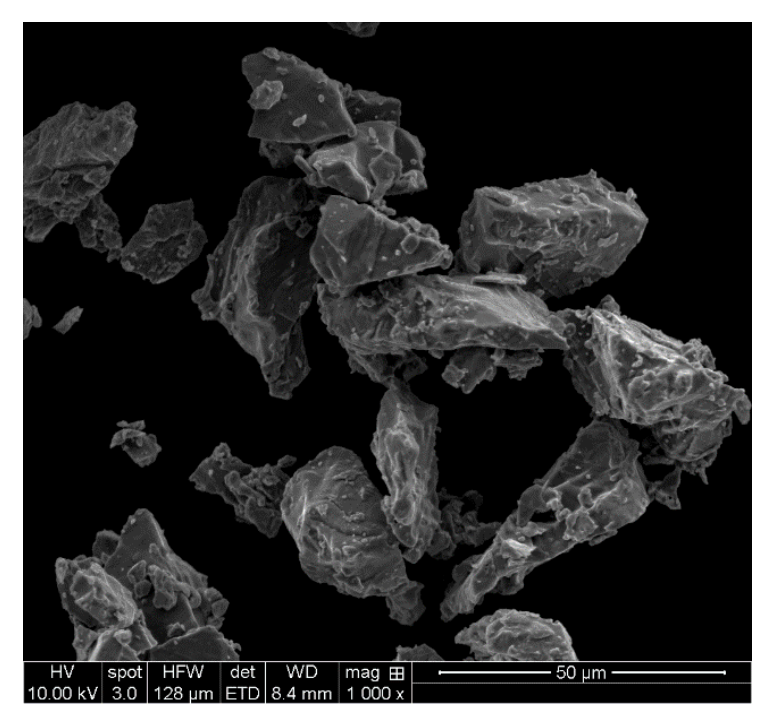

Figure 4-22 : Titanium- Control Sample

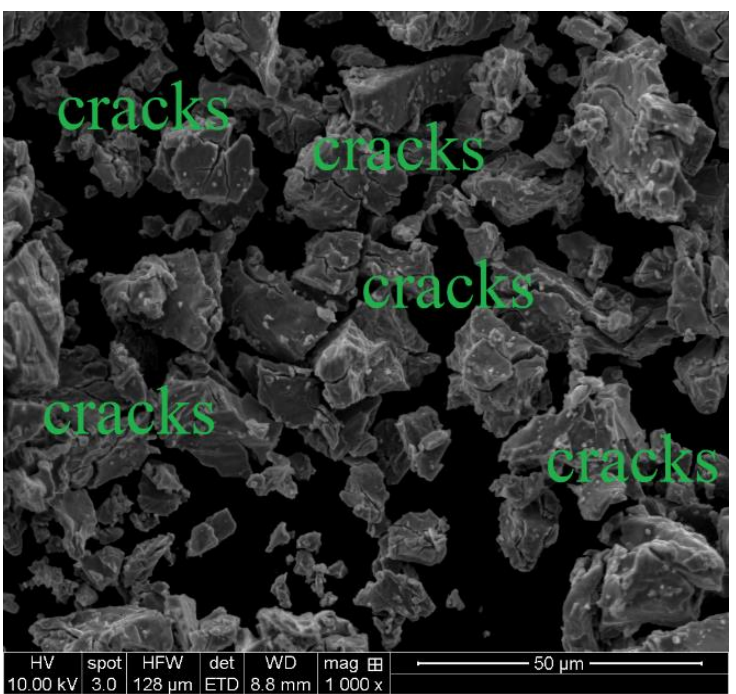

Figure 4-23: Titanium-Experimental Sample

X-ray diffraction (XRD) analysis was performed to understand the structural morphological of the titanium powder after loading. The XRD was performed using a Rigaku 
powder diffractometer operating at a voltage of $40 \mathrm{kV}$ with a current of $44 \mathrm{~mA}$ using a $\mathrm{Cu} \mathrm{K \alpha}$ radiation. Diffraction patterns were measured in the $2 \theta$ range from $30-90^{\circ}$ with a step size of 0.0050. X-ray diffraction pattern for the experimental sample is shown in figure 4-25. The diffraction pattern shows that titanium deuteride was formed during the experiment, thus indicating that deuterium has been absorbed into titanium lattice. Figure 4-24 and figure 4-25 shows the diffraction pattern of titanium (control sample) and titanium deuteride (experimental sample). At room temperature, titanium has a hexagonal close packed structure. This phase is known as the $\alpha$-phase and has a lattice parameter such that $c / a=$ 1.587. The $\alpha$-phase titanium is shown in figure 4-24. The insertion of deuterium atom into titanium lattice increases the $c / a$ ratio. The $\alpha$-phase is stable at $\operatorname{TiH}_{x}(x \ll 0.1)$. Higher deuterium loading $(0.1 \gg x \ll 0.44)$ leads to the $\beta$-phase. Further increase in the deuterium concentration leads to the $\gamma$-phase, which later produces the $\delta$-phase. The $\delta$-phase which has a face-centered cubic lattice (f.c.c) occurs at hydrogen concentration, $x(0.5 \leq x \leq 0.66)$. All the reflections in the titanium deuteride produced during this experiment can be index as belonging to the $\delta$-phase. This indicates that the titanium lattice has absorbed huge amount of titanium. The phase generally results with titanium hydride $\left(T i D_{1.5}\right)$ or higher concentration of hydrogen. Clearly, these peaks do not belong to $T i D_{2}$. The titanium deuterium system is a reversible process and as such during shocking much of the deuterium atom should be released from titanium lattice and will collide with one another. The structural transformation resulting from phase transition leads to redistribution of deuterium atoms which tends to reduce the Columbic barrier and increase the probability of titaniumdeuterium interaction. 


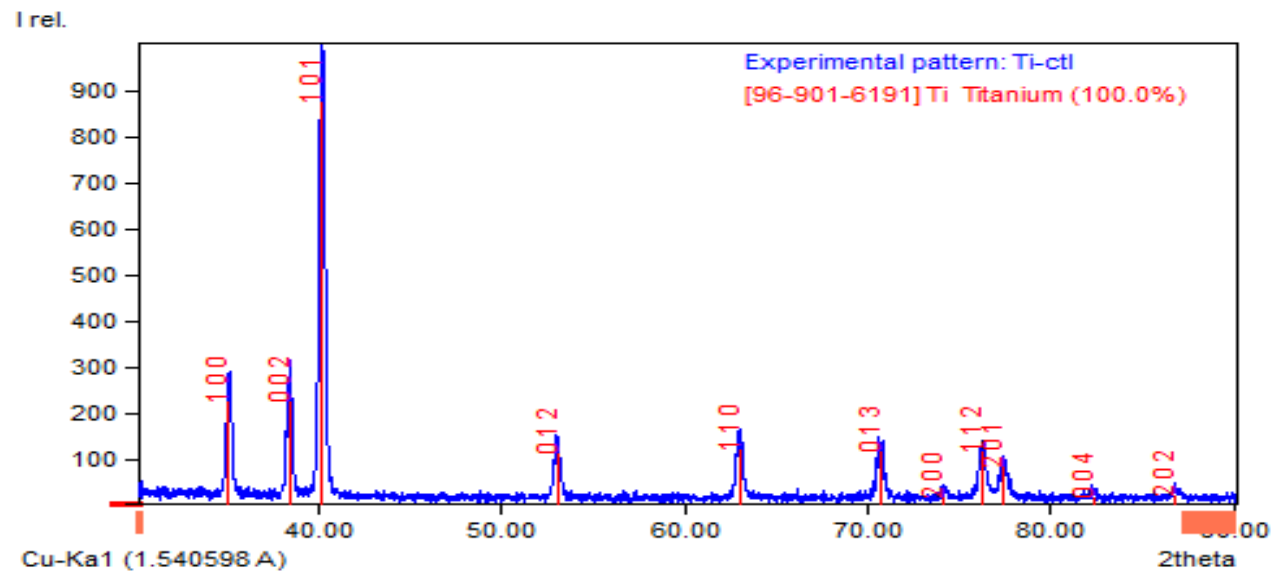

Figure 4-24: XRD pattern of $\alpha$-titanium sample (control experiment).

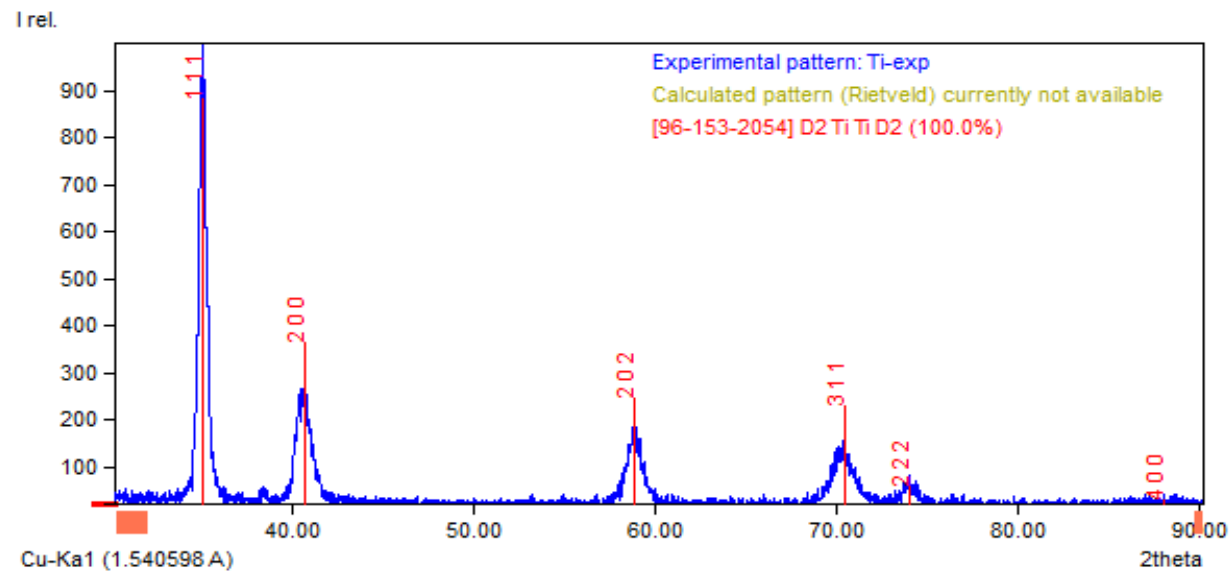

Figure 4-25: X-ray Diffraction (XRD) pattern showing titanium deuteride formation

The titanium powder used in our experiments contains small quantities of oxygen which could lead to oxidation in the thermal shock system resulting to an increase in temperature. It was suggested that this could be the source of heat measured during the experiment. To investigate whether the temperature increase was due to oxidation, titanium samples were heated in a thermo gravimetric analysis (TGA) device. Two samples were used for this investigation. One of the samples was heated in the presence of oxygen, while the other sample was heated under a helium atmosphere at a temperature of $300^{\circ} \mathrm{C}$ for 24 hours. These samples were later analyzed using the scanning electron microscope. The results are in figure 4-26 to figure 4-30. The images do not show that any cracks were produced in the sample, 
therefore eliminating the fact that the increase in temperature observed in thermal shock experiment was due to oxidation. The thermal shock experiment was also conducted with the chamber exposed to air while loading deuterium. Again, no evidence of increase in temperature was noticed during the experiment, further confirming that the increase in temperature observed in experiment 2 was not due to the presence of oxygen in the thermal shock system.
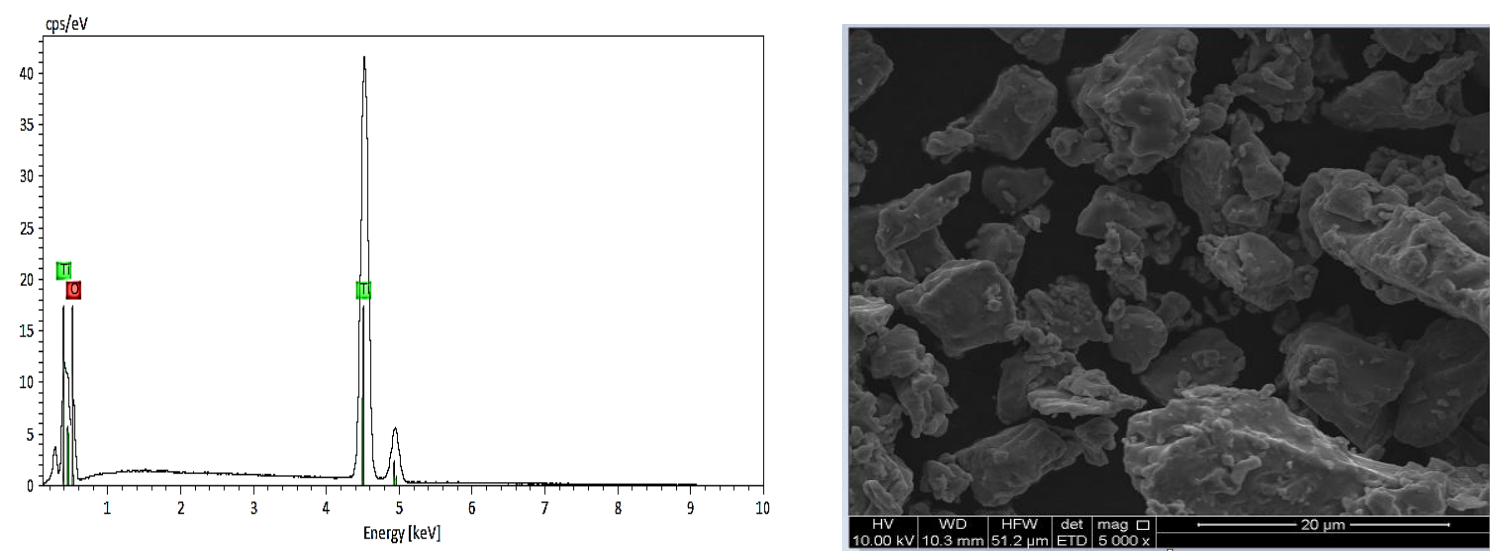

Figure 4-26: Titanium powder (Ti-88.3512 and O: 11.6488)

Figure 4-27: Surface Morphology of sample

Titanium powder heated in the presence of air in TGA equipment at $300^{\circ} \mathrm{C}$
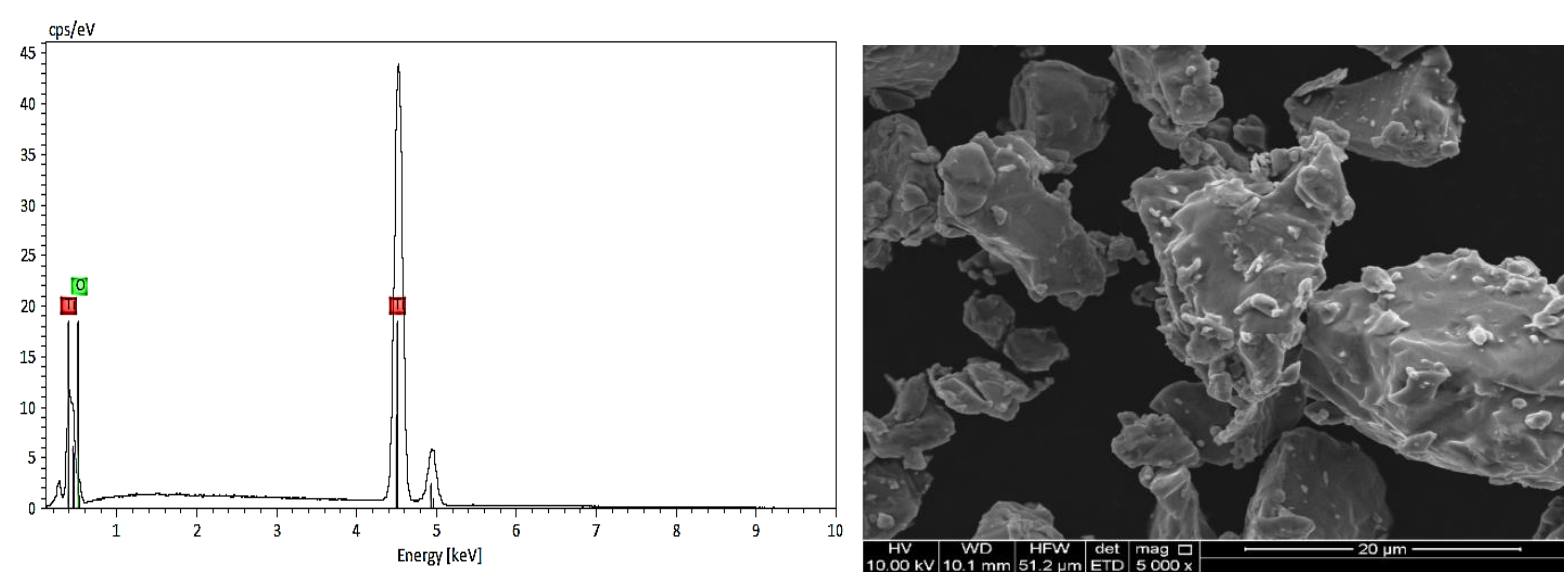

Figure 4-28: Titanium powder (Ti: 95.9117 O: 4.08883)

Figure 4-29 : SEM of sample

Composition of titanium loaded with deuterium in the presence of air. 


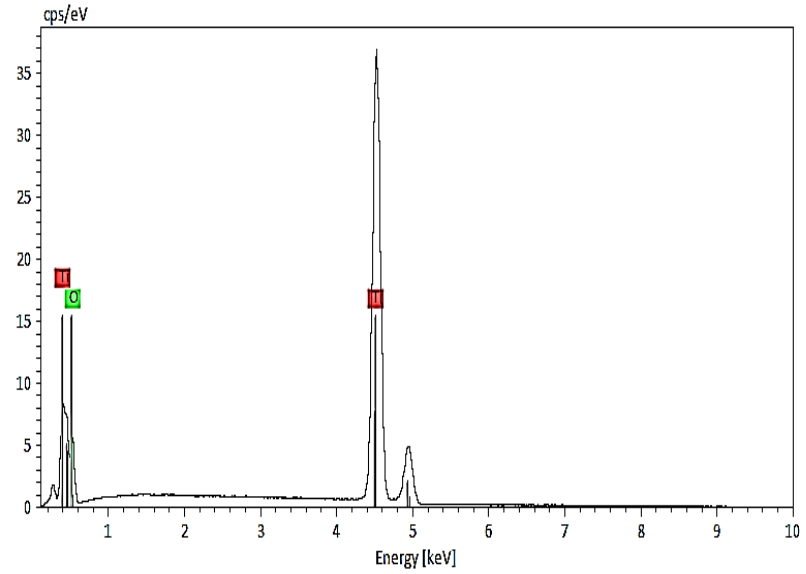

Figure 4-30: Titanium sample (Ti:82.63 0:13.71 C:3.67)

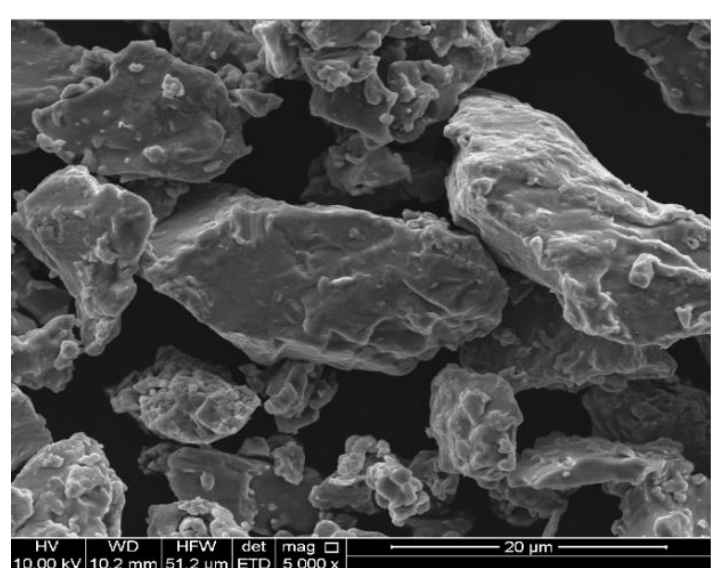

Figure 4-31: SEM image

Titanium loaded with helium control gas in a TGA instrument up to a temperature of $300^{\circ} \mathrm{C}$

The conclusion from this experiment is that the scanning electron microscope analysis confirms the development of cracks in titanium crystal meanwhile x-ray diffraction analysis indicates the formation of titanium deuteride following the thermal shock experiment. Titanium undergoes phase transition during deuterium loading. The phase transition occurs due to huge loading of deuterium into the interstitial spaces of titanium. Cracks produced are consistent with what should be observed during fracto-fusion. The fracto-fusion mechanism suggests that a nuclear effect occurs in condense matter because of crack formation, which can generate sufficient electric field to accelerate deuterons. Cracks appeared at different locations throughout the sample. No neutron emission above background was observed in our experimental system. Our observations of titanium samples from thermal shock systems using high resolution SEM and x-ray diffraction analysis indicate that neutron emission from titanium-deuterium systems may not be a direct result of the fracto fusion mechanism.

\section{EXPERIMENT 3}

The procedure for running experiment 3 is like that used in experiment 2 . The sample used in this experiment was dehydrided titanium -325 mesh, $99.99 \%$ purity with mass $\mathrm{f} 13.6$ 
g. The composition of the sample analyzed using EDS is shown in figure 4-32 and table 4-3.

The sample contains $98.8 \%$ titanium and a small amount of carbon, $1.2 \%$.

Table 4-3: Composition of Titanium used in the Experiment 3

\begin{tabular}{|c|c|c|c|c|}
\hline $\begin{array}{c}\text { Elemental } \\
\text { Comp. }\end{array}$ & $\begin{array}{c}\text { Atomic } \\
\#\end{array}$ & $\begin{array}{c}\text { Mass } \\
{[\%]}\end{array}$ & $\begin{array}{c}\text { Mass } \\
\text { Norm. [\%] }\end{array}$ & $\begin{array}{c}\text { Abs. } \\
\text { error [\%],1 } \sigma\end{array}$ \\
\hline Titanium & 22 & 1101.20 & 98.79 & 3.50 \\
\hline Carbon & 6 & 1.24 & 1.21 & 0.21 \\
\hline
\end{tabular}

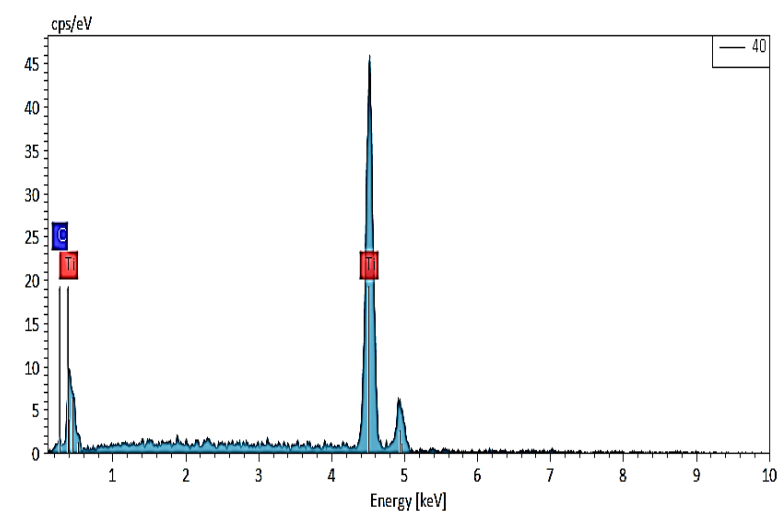

Figure 4-32: Titanium composition in Titanium powder

Long background counts were recorded with the LabVIEW program every $0.01 \mathrm{~ms}$ for a period of one week, with the program restarted every 24 hours. The background counts before running the experiment (counts $/ 0.001 \mathrm{~ms}$ ) as a function of time is shown in figure 4.33 and figure 4.34. A moderated, an unmoderated helium-3 detector and a proton recoil detector were used to monitor neutron emissions during this experiment. The maximum observed background count rate for the moderated and unmoderated ${ }^{3} \mathrm{He}$ detector was 2 and the count rate was zero in the proton recoil detector. 


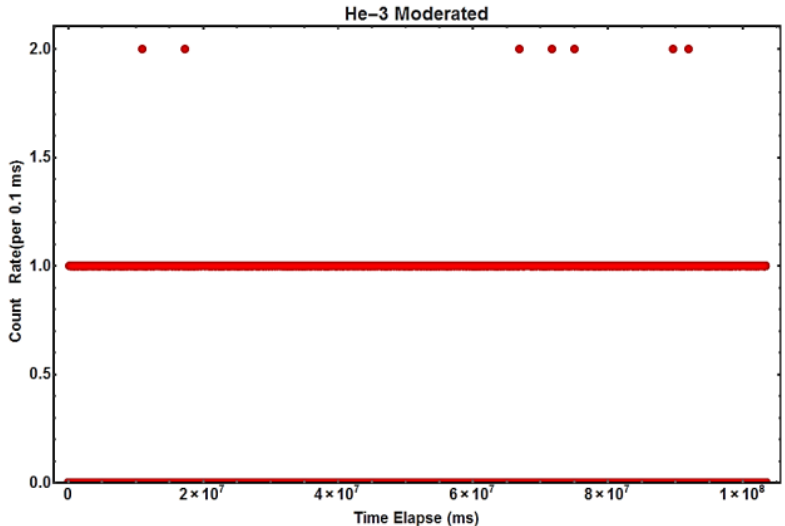

Figure 4-33: Moderated ${ }^{3} \mathrm{He}$-Background Count Rate

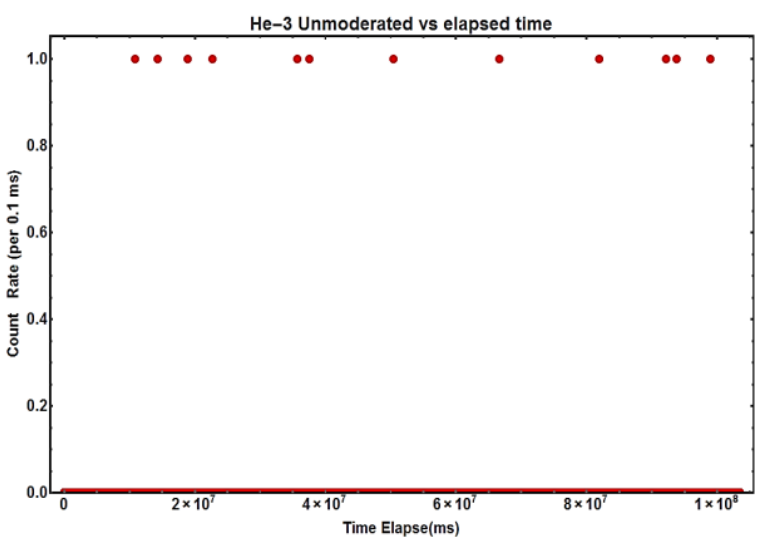

Figure 4-34: Unmoderated ${ }^{3} \mathrm{He}$-Background Count Rate

Figure 4-35 and figure 4-36 shows the temperature of the inside chamber and ambient temperature of the experimental system with time before the system was placed in liquid nitrogen and after transferring the system into boiling water. The system was thermally shocked in boiling water after leaving it in liquid nitrogen for about 6 hours. Figure 4-37 shows the variation in pressure of the experimental chamber during this time.

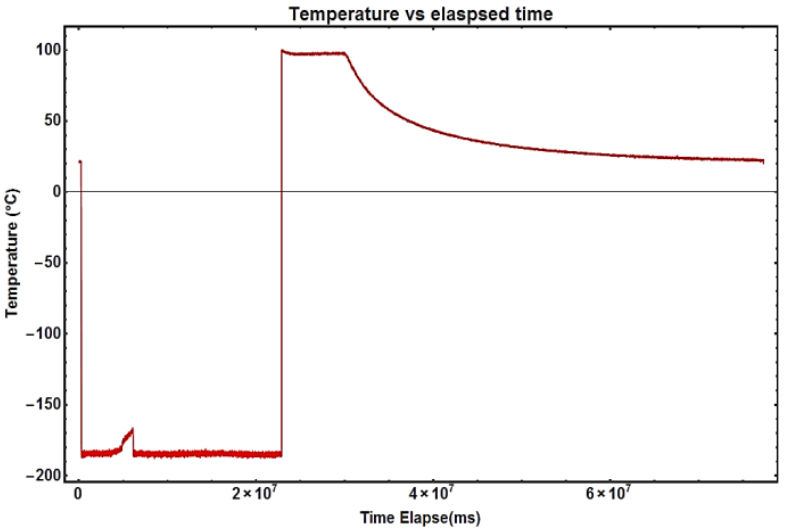

Figure 4-35: Inside Temperature vs time

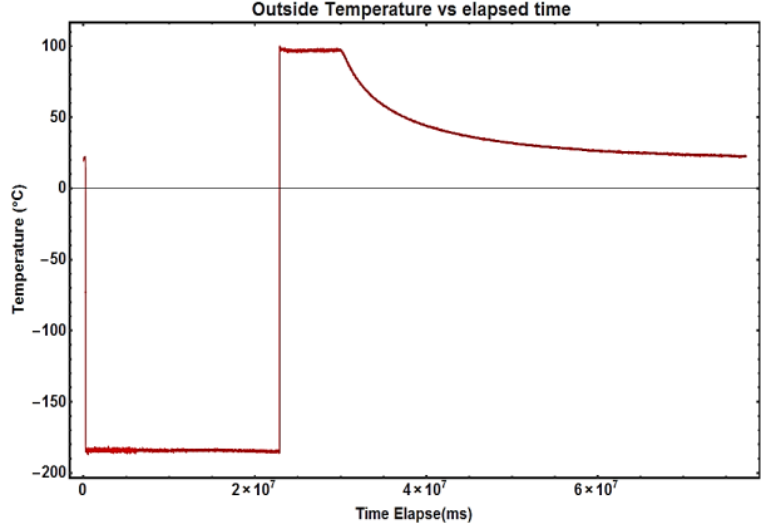

Figure 4-36: Outside Temperature vs time 


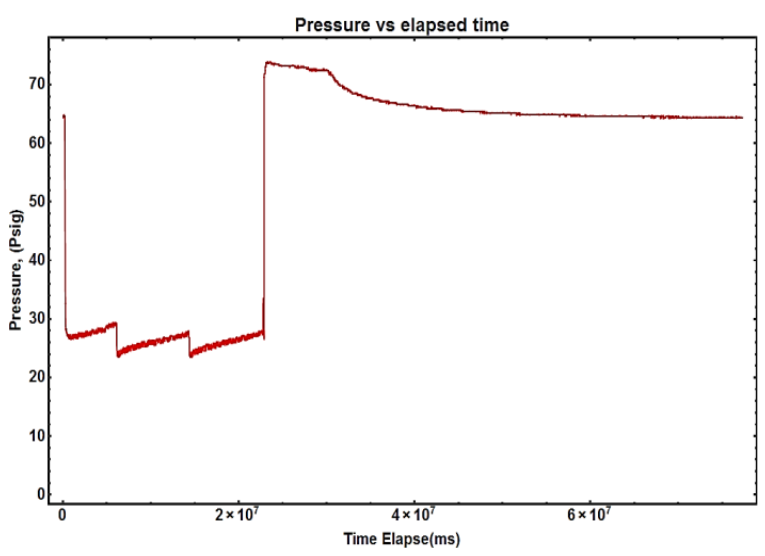

Figure 4-37 : Pressure variation with time
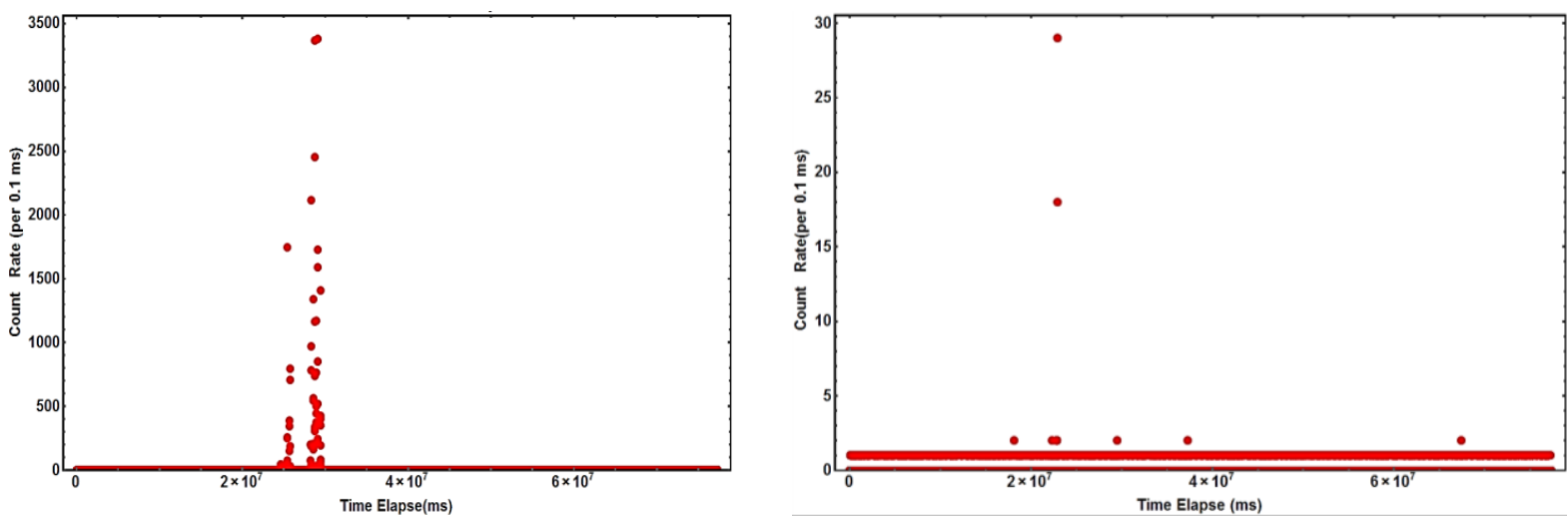

Figure 4-38: Unmoderated ${ }^{3} \mathrm{He}-$ Foreground Count (noise)

Figure 4-39: Moderated ${ }^{3} \mathrm{He}$-foreground (noise)

Our experimental system was insulated with a cotton foam. Cotton foam leaks nitrogen because it is not a good insulating material. As a result, the system had to be replenished with liquid nitrogen every 1-2 hours. This is reflected on the graph by the regions showing the small dips and rise on the pressure plot. During this experiment, we observed no significant increase in counts from the proton recoil detector. However, there was a significant neutron burst observed in the moderated ${ }^{3} \mathrm{He}$ detector (figure 4-39), just after shocking the experimental chamber, with a total count rate of 2,548 and maximum at 29 counts/100ms. A huge number of neutrons with a total count rate of 36,375 and maximum at 3,384 counts/100ms were recorded in the unmoderated detector (figure 4-39) during this period. The difference between the count rate in the two helium detectors is because neutrons are being thermalized by the polyethylene sleeve surrounding the moderated detector. Since no 
neutron burst was observed in the proton recoil detector, we hypothesized that the emitted neutrons should be thermal or cold neutrons. To investigate our hypothesis, the unmoderated ${ }^{3} \mathrm{He}$ detector was exposed to Cf-252 source for over 2 hours (figure 4-40). The neutron source has a mass of $3.84 \times 10^{-2}$ ugm and produces $2.3 \times 10^{6} \mathrm{n} / \mathrm{s} / \mathrm{ugm}$. No significant neutron counts were observed from Cf-252 source, further confirming the fact that the neutrons observed during the thermal shocking procedure were thermal or cold neutrons.

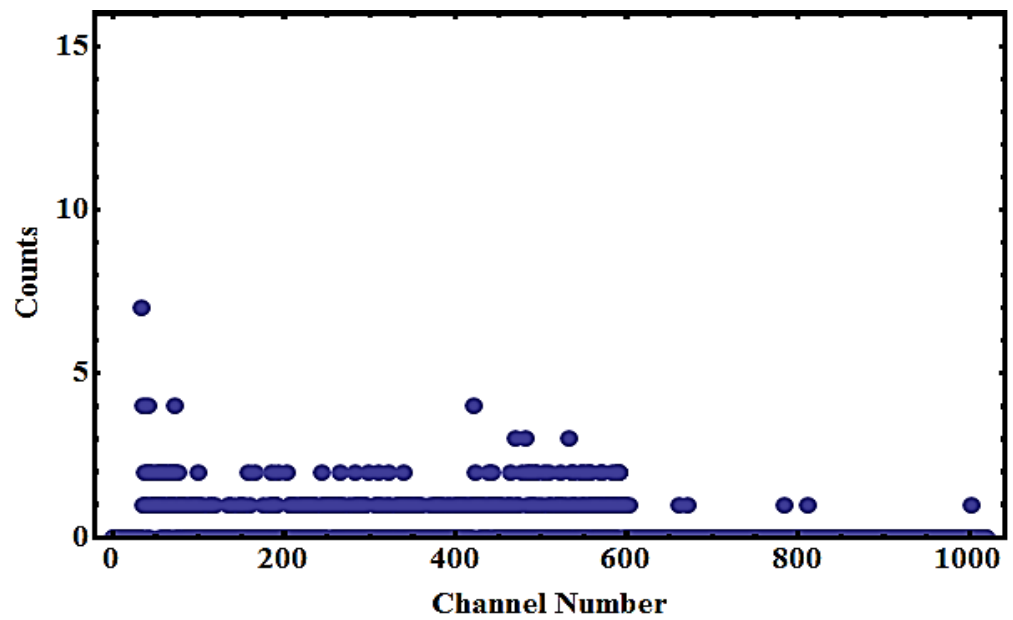

Figure 4-40: Observed count rate from Cf-252 - unmoderated Helium-3 detector

In the context of these discussions, it is important to mention that Widom and Larsen have proposed that ultra-low momentum neutrons could be produced via weak interactions. This theory provides an explanation as to how ultra-low momentum neutrons are produced from thermal shock systems. According to Widom-Larsen theory, electromagnetic energy stored in slow-moving electrons under certain conditions can be collectively transferred into fewer, much faster electrons with kinetic energy strong enough to combine with a proton or deuterium via weak interaction, to produce ultra-low momentum neutrons. These neutrons are easily absorbed by surrounding media because of their high neutron cross section. The production of ultra-low momentum neutrons can induce chains of nuclear reactions in neighboring condensed matter. Very few of these neutrons will leave the vicinity and will 
really be experimentally detected [1-3]. In a thermal shock system, a pressure difference caused by a rapid temperature change across a palladium surface will pack the surface layer to a single compact layer allowing for the required coherent electric field producing motions. The surface temperature increases under such circumstances when the system is shocked, creating a non-equilibrium state which is required for the electrons to combine with deuteron.

The Monte Carlo N particle code, MCNPX [4] was used to investigate the slow energy neutrons detected in this experiment. The study involves modelling the response of a helium-3 detector from an isotropic neutron point source after thermalization from boiling water. The helium-3 detector has a radius of $1.85 \mathrm{~cm}$ and height of $13.5 \mathrm{~cm}$ (unmoderated helium-3 detector), and is enclosed in a polyethene sleeve with radius of $31 \mathrm{~cm}$ and height of $12 \mathrm{~cm}$ (moderated helium-3 detector). The system was modelled as close as possible to our experimental design. The geometry consisted of a point source at the center of a cylindrical water bath made with steel walls, with the neutron source placed about $27 \mathrm{~cm}$ from the detectors. About $1 \times 10^{6}$ particles were run to produce good statistics. A monoenergetic neutron source was used in these simulations. Two cases were modeled, including: 1). The first simulation involves a $2.45 \mathrm{MeV}$ neutron source placed $27 \mathrm{~cm}$ from the moderated and unmoderated detectors. As expected the neutrons did not deposit enough energy in the unmoderated helium-3 detector, meanwhile a large neutron flux was observed in the moderated helium-3 detector. The ratio of counts in the moderated to unmoderated He-3 detector is $\sim 327$. When a $0.025 \mathrm{eV}$ was used in the simulation, the ratio of counts in the unmoderated to moderated detector was $\sim 12,2$ ). The second case involved modelling the actual experimental design. Here, the neutron source was placed at the center of a cylinder with radius of $13.5 \mathrm{~cm}$ and height of $25.4 \mathrm{~cm}$, and filled with water. The ratio of counts in the unmoderated detector to the moderated detector is $\sim 1.27$ for a $0.025 \mathrm{eV}$ neutron and 0.85 for $2.45 \mathrm{MeV}$. 
These results imply that boiling water should not be used for shocking the system to prevent scattering of slow neutrons before they are detected by the unmoderated helium-3 detector. The results of neutrons detected in this experiment is rule out as noise but provide the basis for future experiments. As a result, in the next series of experiments, a heat gun was used to create the non-equilibrium condition.

The samples used in this experiment were analyzed using the SEM to determine any change in the morphology of titanium powder before and after the experiment. No surface change in the surface morphology of the sample was observed.

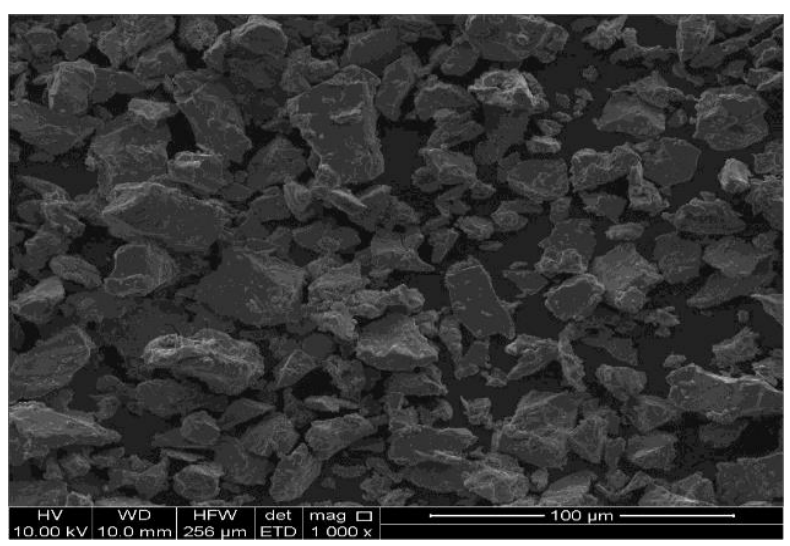

Figure 4-41: SEM Images of control

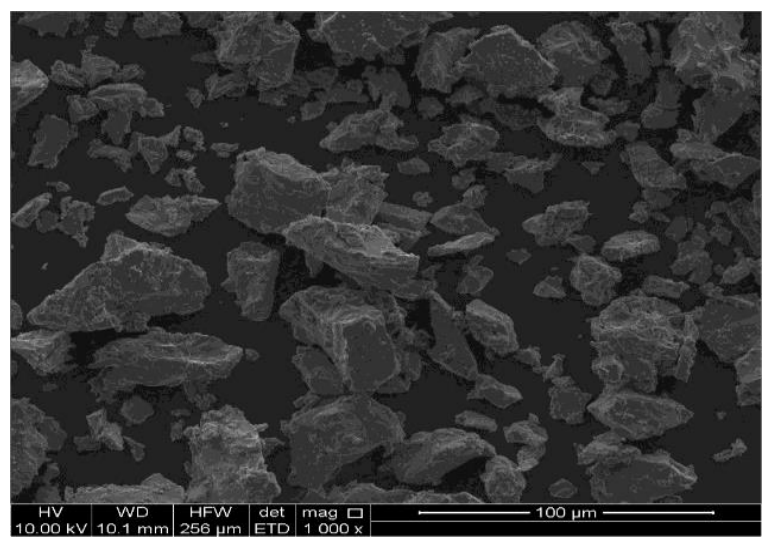

Figure 4-42: SEM Images-Experimental Sample

\subsubsection{Shocking by means of a Heat Gun}

This basis for this procedure was from the results in experiment 3 . In the series of experiments that follow, after titanium powder was removed from liquid nitrogen, an electric heat gun was used to thermally shock our system. The results from these experiments are summarized below. 


\section{Experiment 4}

The mass of titanium used in this experiment was $14.76 \mathrm{~g}$. The composition of the titanium used in this experiment is shown in the figure below:

Table 4-4: Composition of Titanium

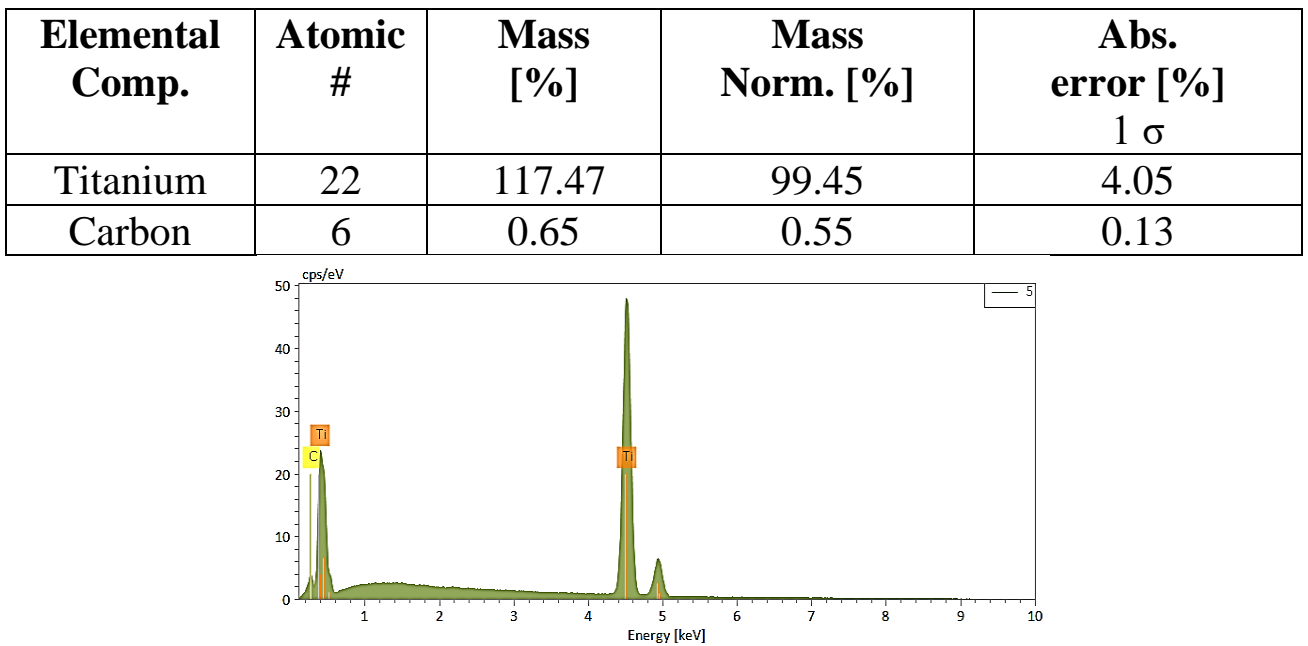

Figure 4-43: Composition of Titanium

The background count rate before the experimental run is shown in figure 4-48 and figure 4-

49. The maximum count rate is 2 counts/ $0.001 \mathrm{~ms}$ observed in the unmoderated and moderated helium-3 detector.

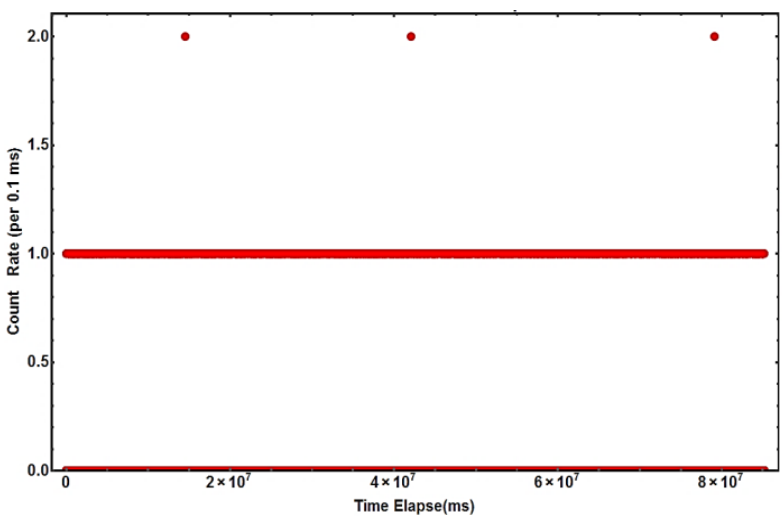

Figure 4-44: Moderated He3 -Background Count

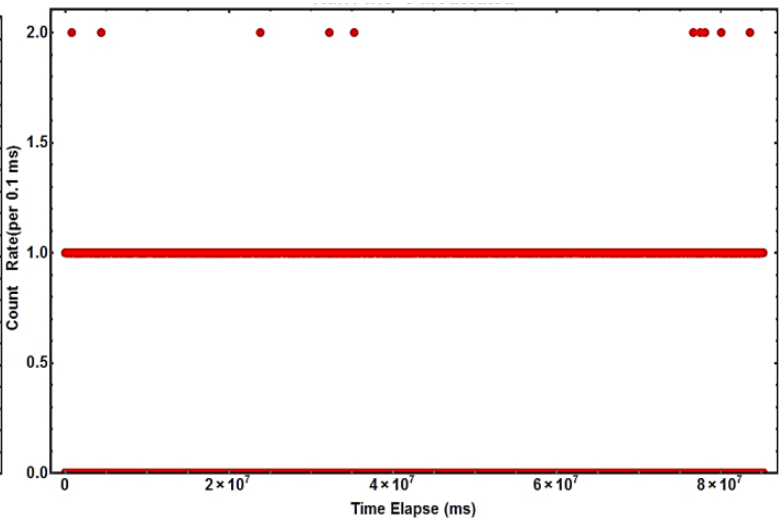

Figure 4-45: Unmoderated He3 -Background Count 
The graph in figure 4-50 and figure 4-51 shows the variation of temperature of the inside and outside thermocouples with time, while the variation in pressure during the experiment is shown in figure 4-52. A heat gun was used to rapidly heat up the experimental system after removing it from liquid nitrogen. During this experiment, a very small neutron burst was observed in both the moderated and the unmoderated helium-3 detector with maximum count rates of 30 and 10 counts $/ 0.001 \mathrm{~ms}$, respectively.

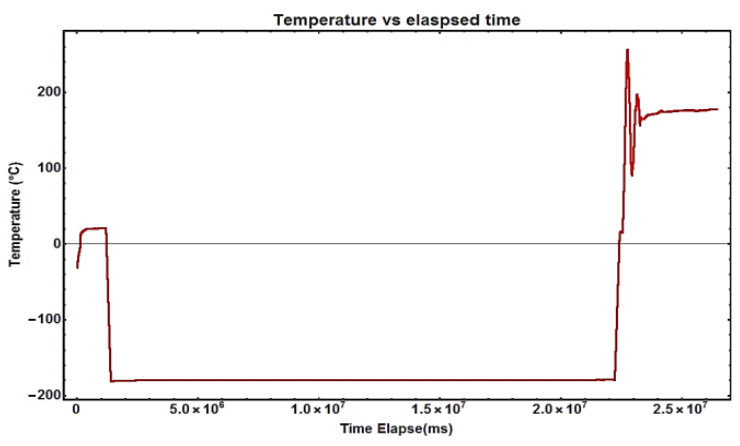

Figure 4-46: Inside Temperature vs time

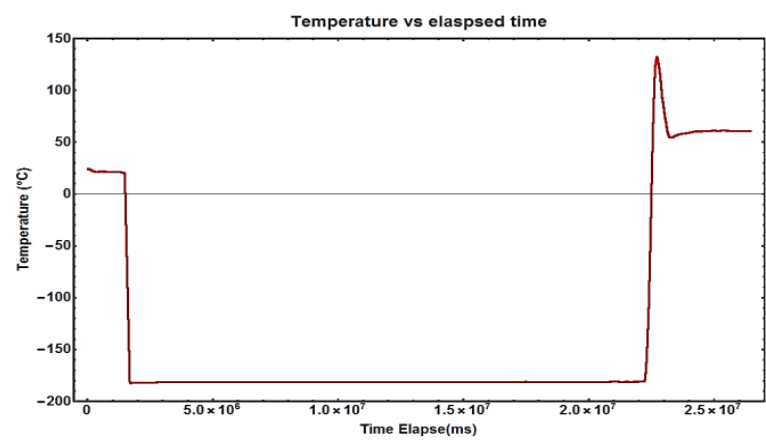

Figure 4-47: Outside Temperature vs time

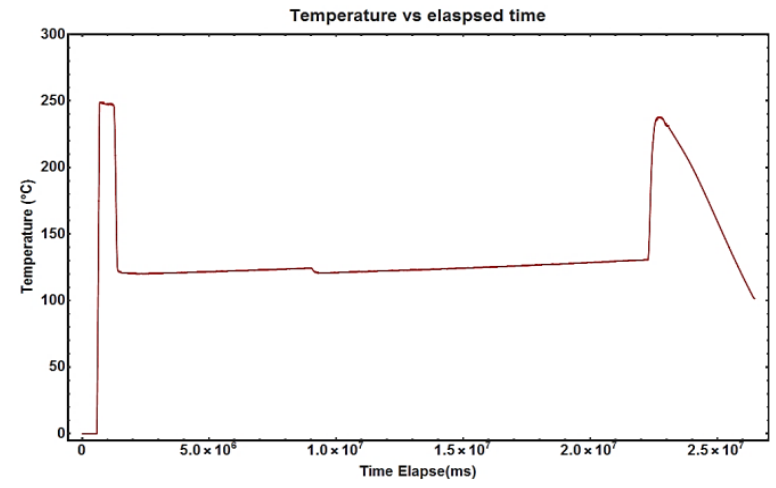

Figure 4-48: Pressure Variation with time during and after shocking

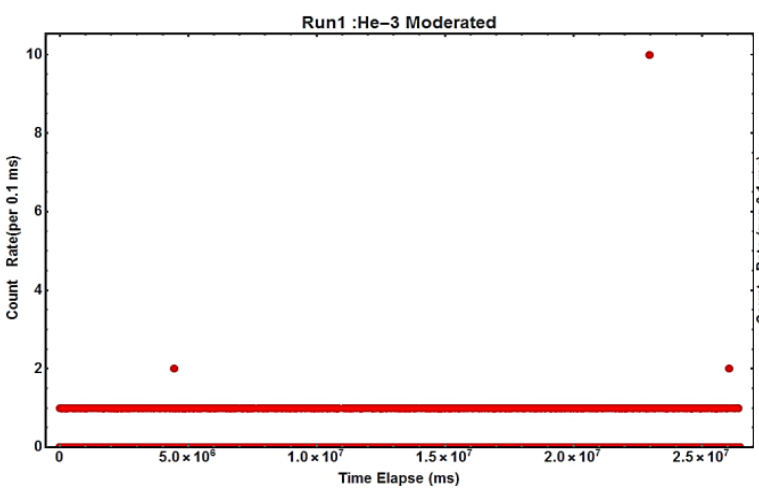

Figure 4-49: Moderate ${ }^{3} \mathrm{He}$ - Foreground Count

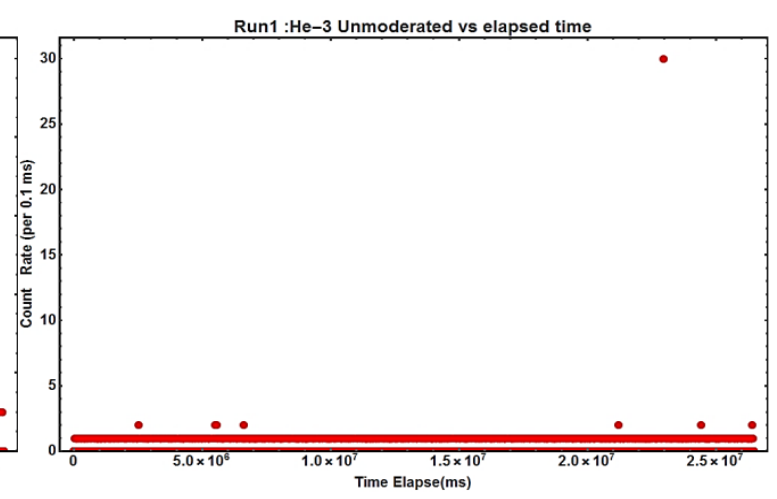

Figure 4-50: Unmoderated ${ }^{3} \mathrm{He}$ - Foreground Count 
The surface morphology of the samples after the experiment is shown in figure 4-55 and figure 4-56. The experimental sample shows crack formation throughout the sample due to deuterium absorption during loading. These cracks are not uniform throughout the sample; occurring in certain areas of the sample, and not in others. This implies that if a titaniumdeuterium reaction was to occur, it would occur at certain areas in the sample. The formation of cracks in certain areas and not in others negatively affect titanium-deuterium reaction. Hence, the reaction occurring in titanium deuterium systems is a local phenomenon. The neutron burst observed in this experiment is likely associated with crack formation since the burst occurs long after shocking the experimental system.

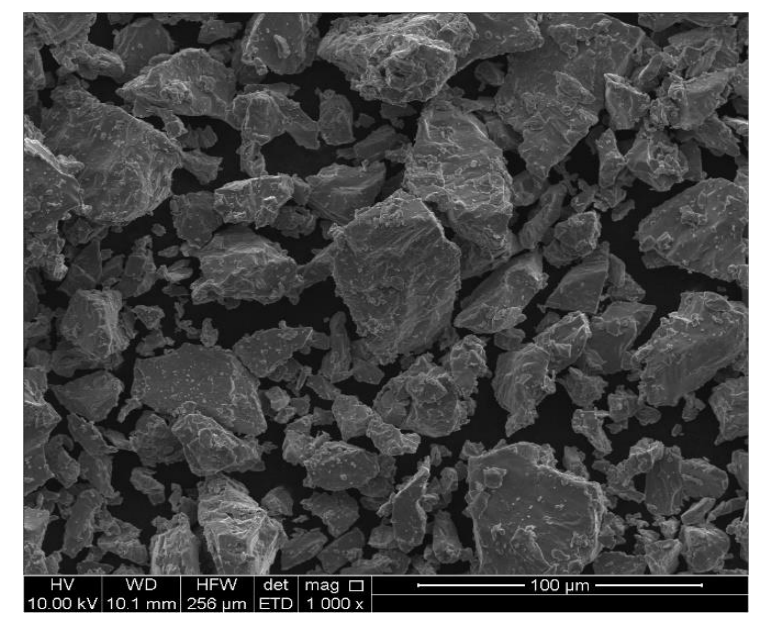

Figure 4-51 : SEM Image-Control Sample

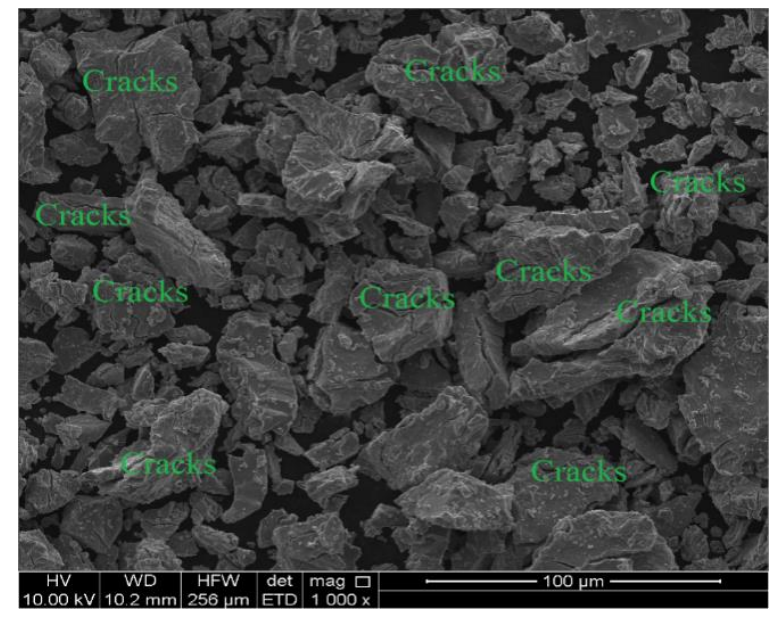

Figure 4-52: SEM Image-Experimental Sample

\section{EXPERIMENT 5}

The mass of the titanium powder was increased from the usual $15 \mathrm{~g}$ to $42 \mathrm{~g}$ to investigate the influence of the number of atoms of titanium on the titanium-deuterium reaction. The increase in mass affects the ratio of deuterium to titanium atoms (loading ratio), and therefore increases the number of sites available for the reaction to occur. The titanium powder was a -325 mesh (99.5\% metal basis) which differs from the sample used in 
experiment 1-4 in that it has a lower purity, it is cheaper, and the particle sizes are much larger. The EDS analysis of this powder is shown in the figure below:

Table 4-5: Composition of Titanium Powder

\begin{tabular}{|c|c|c|c|c|}
\hline $\begin{array}{c}\text { Elemental } \\
\text { Comp. }\end{array}$ & $\begin{array}{c}\text { Atomic } \\
\#\end{array}$ & $\begin{array}{c}\text { Mass } \\
{[\%]}\end{array}$ & $\begin{array}{c}\text { Mass } \\
\text { Norm. [\%] }\end{array}$ & $\begin{array}{c}\text { Abs. } \\
\text { error [\%], 1 } \sigma\end{array}$ \\
\hline Titanium & 22 & 107.92 & 99.03 & 3.72 \\
\hline Carbon & 6 & 1.06 & 0.97 & 0.28 \\
\hline
\end{tabular}

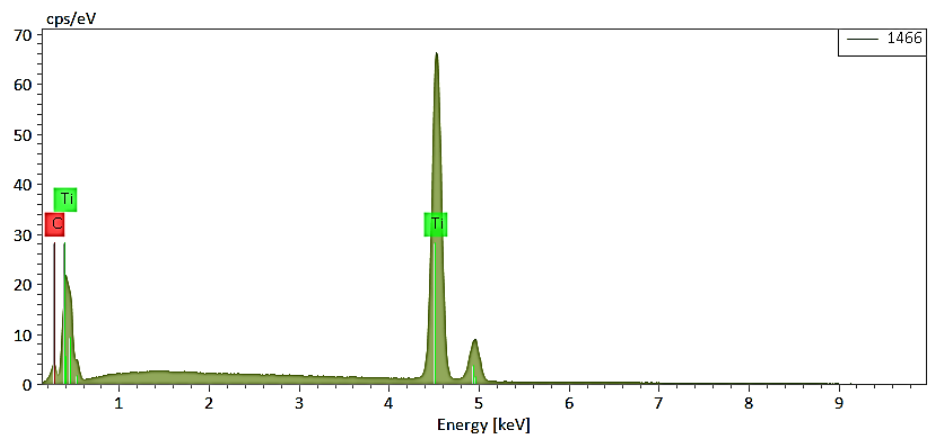

Figure 4-53: Composition of titanium used in thermal shock experiment

Figure 4-62 shows the pressure-time data during this experiment. There was a lot of deuterium absorption in this sample as shown in figure 4-62. Deuterium was introduced in the sample in several cycles. The titanium mesh absorbed about 1450 psig of deuterium in the first 3 days before the system reached equilibrium. During this period, no neutron burst was observed, but rather an increase in temperature of the system was observed. The temperature of the system was not monitored because temperature measurement generally begins just before placing the system in liquid nitrogen. The huge absorption and temperature increase is a result of phase transition that occurs in titanium lattice or the formation of titanium hydride. However, the observed change in color of the experimental system indicates that the temperature must have been above $420^{\circ} \mathrm{C}$. The background count rate of this case is shown in 
the figure 4-58 and figure 4-59. The maximum recorded count rate is 2 counts/100 $\mathrm{ms}$ in both detectors.
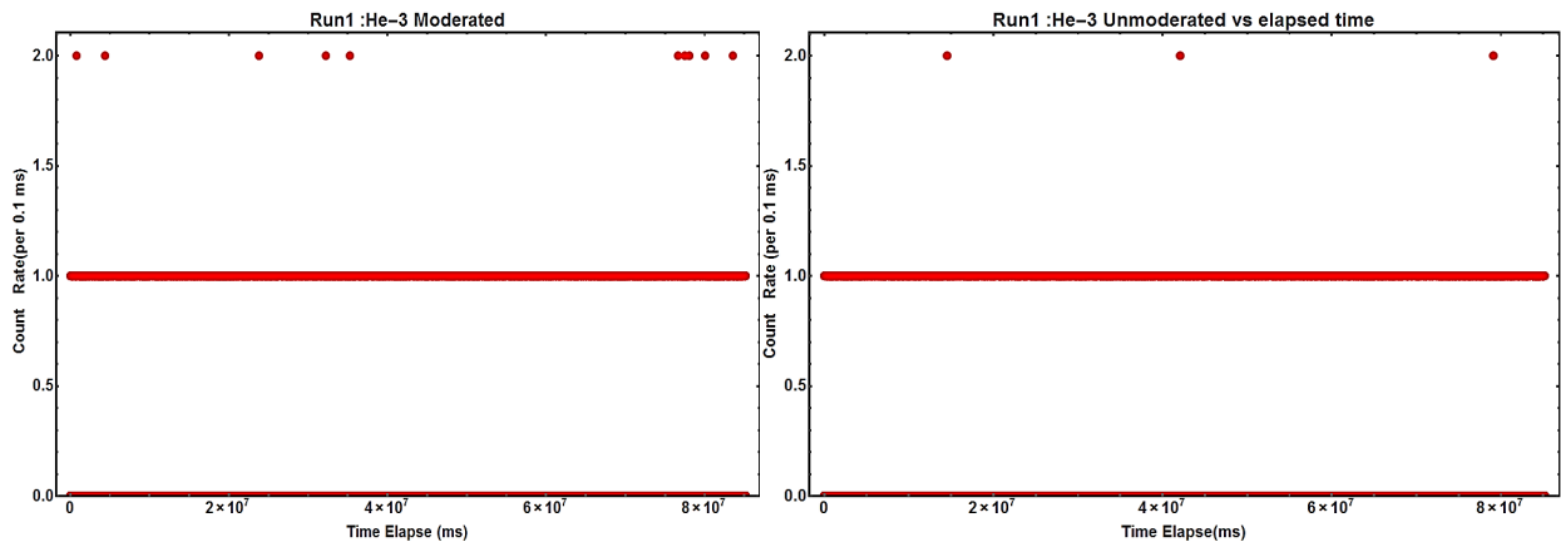

Figure 4-54: Moderated ${ }^{3} \mathrm{He}$ - Background Count

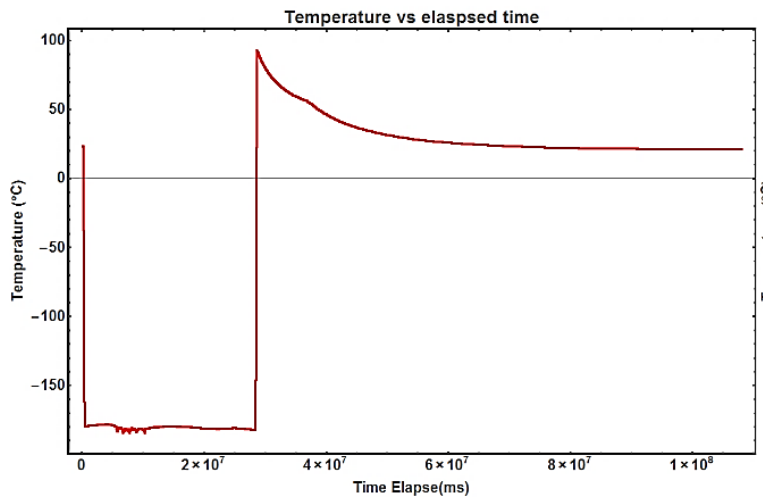

Figure 4-56: Inside Temperature vs time

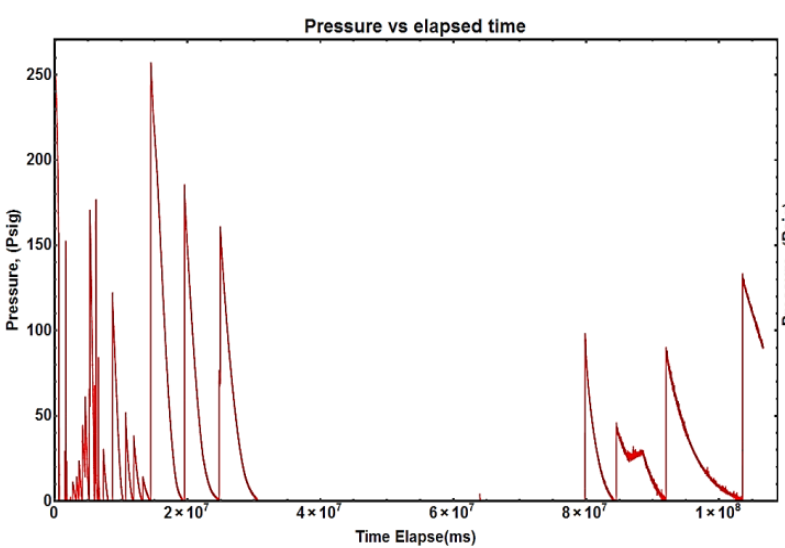

Figure 4-58: Pressure drop during loading
Figure 4-55: Unmoderated ${ }^{3} \mathrm{He}-$ Background Count

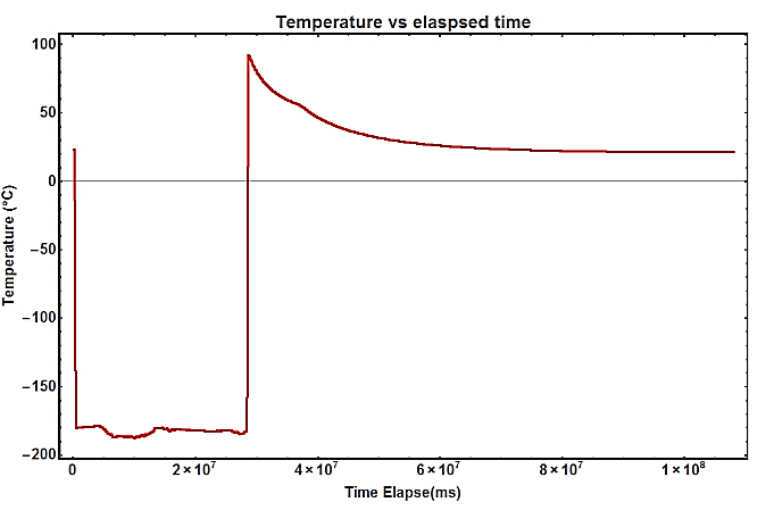

Figure 4-57: Outside Temperature vs time

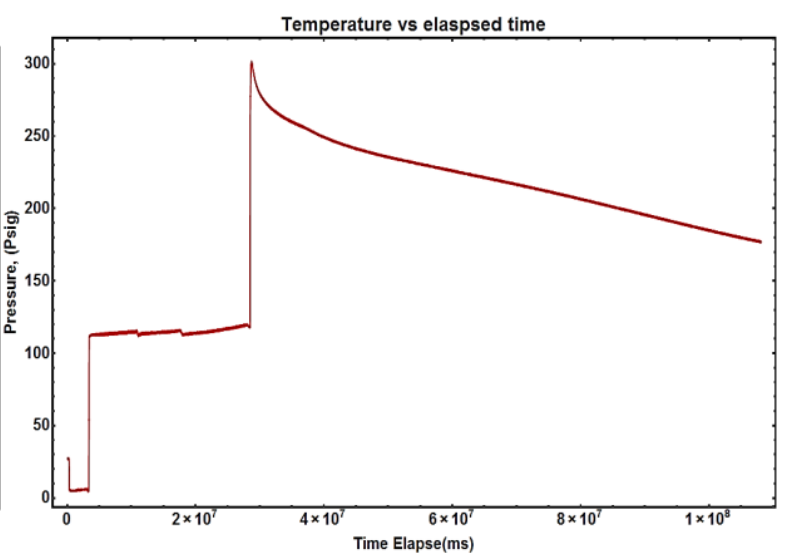

Figure 4-59: Pressure during and after shocking 


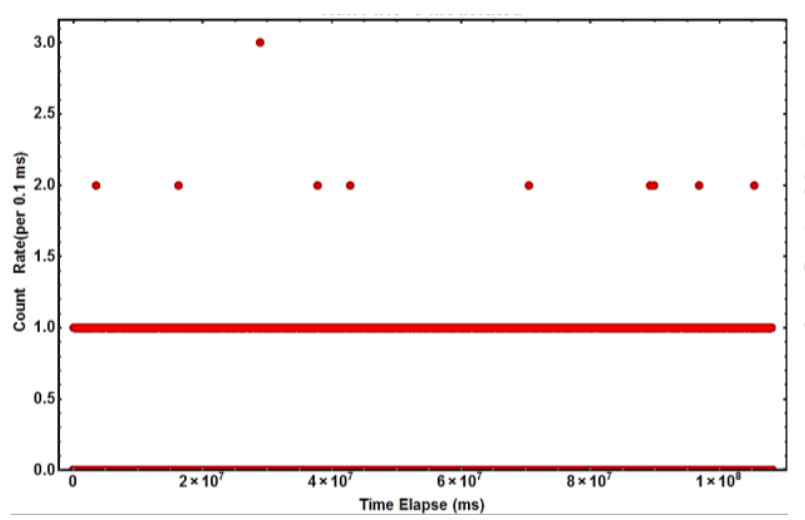

Figure 4-60:Moderated ${ }^{3} \mathrm{He}$ Counts-Foreground Count

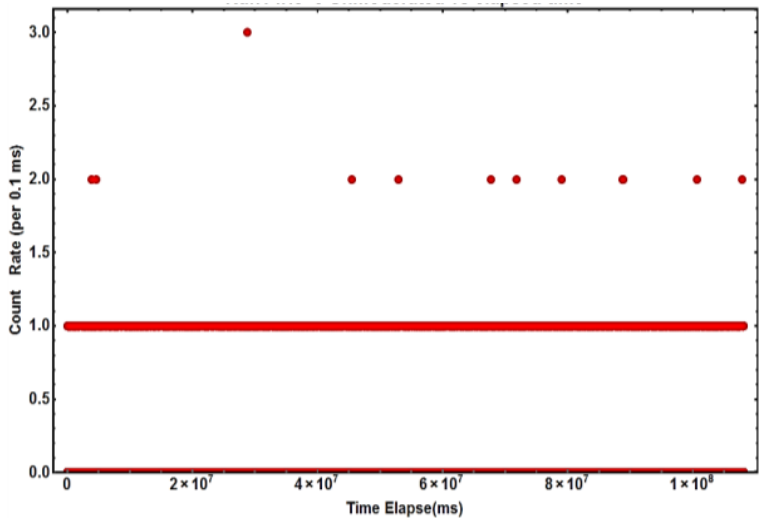

Figure 4-61:Unmoderated ${ }^{3} \mathrm{He}$ Counts-Foreground Count

The titanium samples used in the experimental investigation shows that cracks were formed in titanium lattice from deuterium absorption. The large size of the titanium powder resulted in huge amount of absorption which meant that the titanium powder would require a longer amount of time to reach equilibrium, which is not beneficial for this investigation. As a result, this powder was not used in future investigations. The impurities in the sample greatly affected absorption.

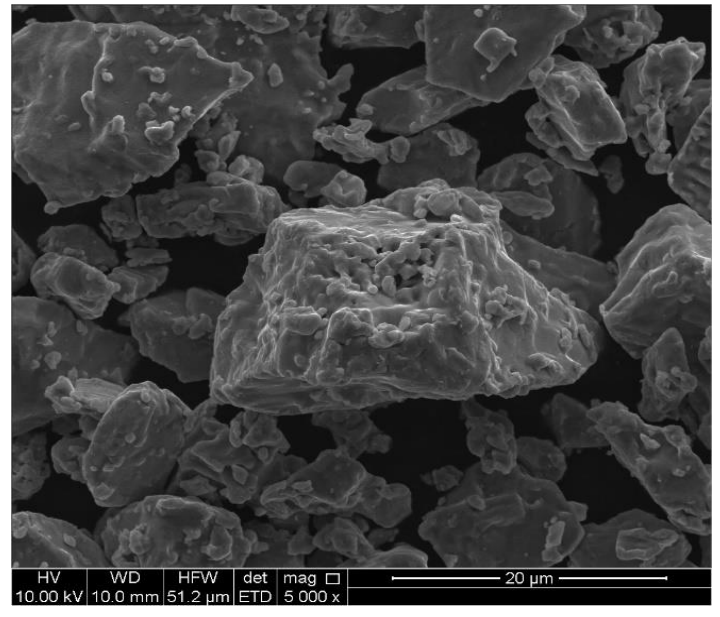

Figure 4-62- Titanium powder- Control Sample

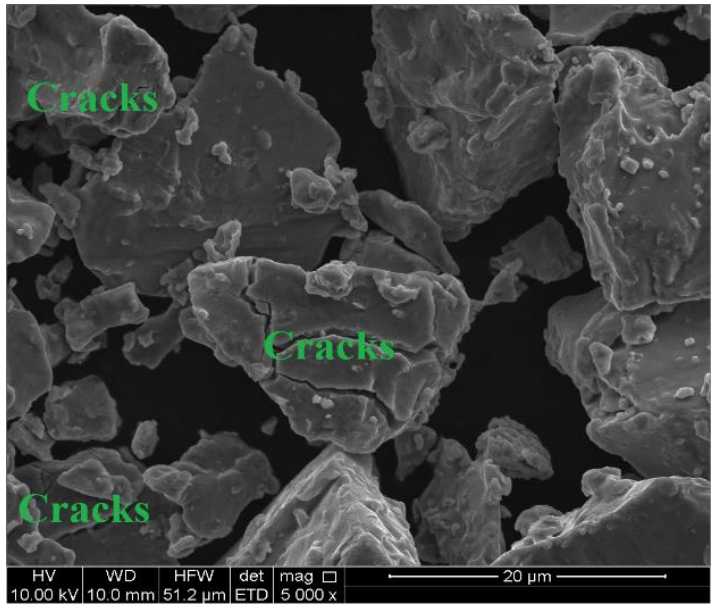

Figure 4-63: Titanium powder- Experimental sample 


\section{Experiment 6}

The mass of the sample used was $5 \mathrm{~g}$. The EDS analysis of the control sample is shown in Table 4-6 and Figure 4-68.

Table 4-6: Composition of control sample 6

\begin{tabular}{|l|c|c|c|c|}
\hline $\begin{array}{c}\text { Elemental } \\
\text { Comp. }\end{array}$ & $\begin{array}{c}\text { Atomic } \\
\#\end{array}$ & $\begin{array}{c}\text { Mass } \\
{[\%]}\end{array}$ & $\begin{array}{c}\text { Mass } \\
\text { Norm. [\%] }\end{array}$ & $\begin{array}{c}\text { Abs. } \\
\text { error [\%],1 } \\
\sigma\end{array}$ \\
\hline Titanium & 22 & 134.14 & 99.43 & 4.62 \\
\hline Carbon & 6 & 0.77 & 0.57 & 0.15 \\
\hline
\end{tabular}

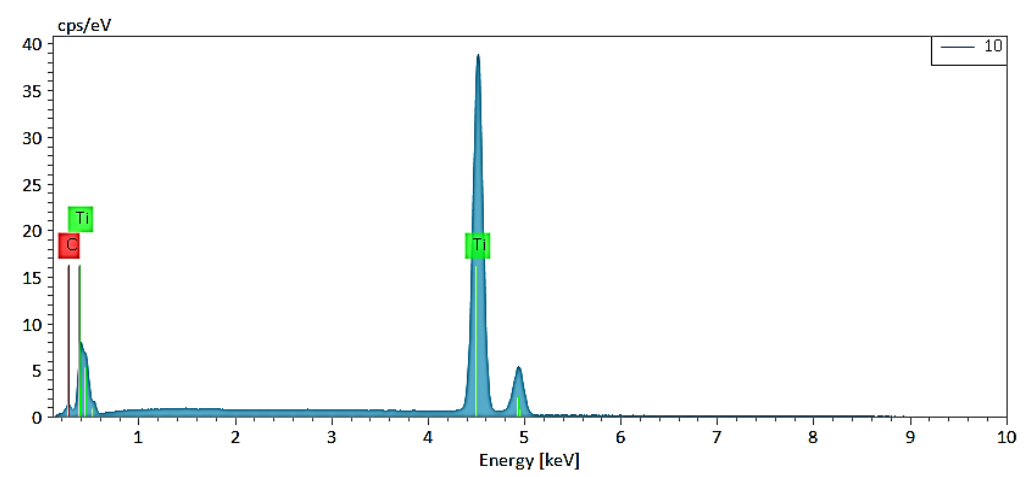

Figure 4-64: Composition of Titanium, Experiment 6

Background counts were recorded in the experiment for a week with the Labview program, with the program restarted every 24 hours. Figure 4-69 and figure 4-70 shows the background counts prior to the experiment. The maximum recorded count rate is 2 counts $/ 0.01 \mathrm{~ms}$ in the moderated and unmoderated helium-3 detectors, respectively. 

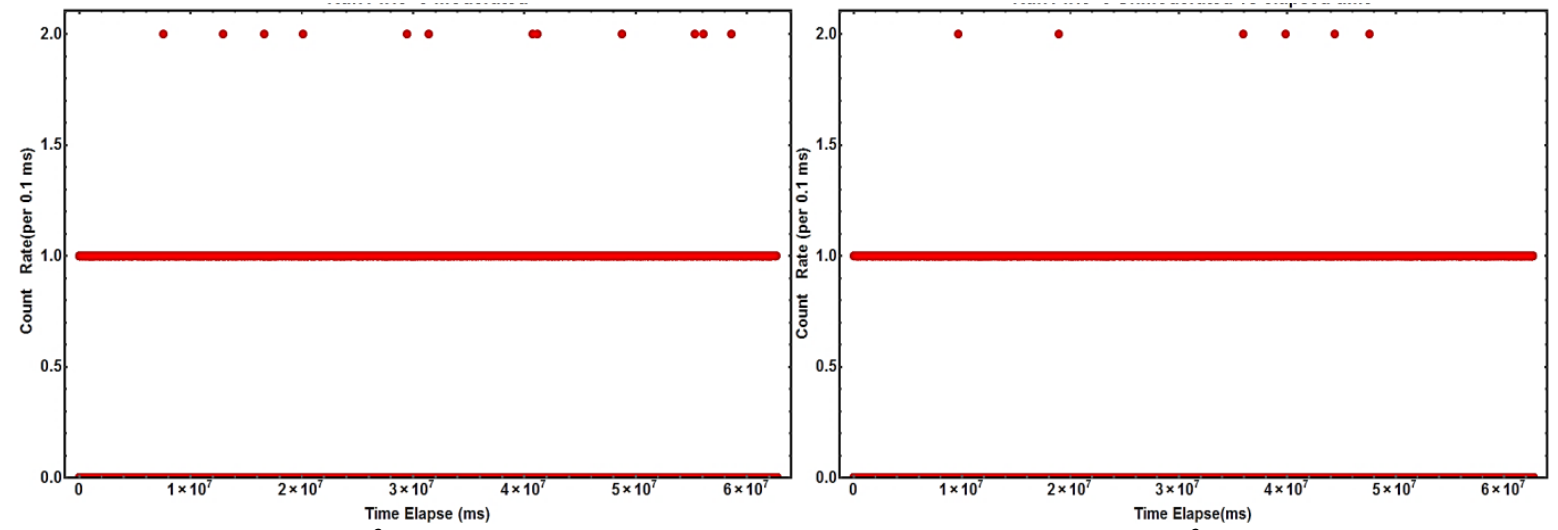

Figure 4-65:Moderated ${ }^{3} \mathrm{He}$ Detector-Background Count Figure 4-66:Unmoderated ${ }^{3} \mathrm{He}$-Background Count

Figure 4-71 shows the temperature of the titanium powder during the experiment. Shocking was achieved by using a heat gun. The pressure graph of the system is shown in figure 4-73. A very small neutron burst was observed during shocking.

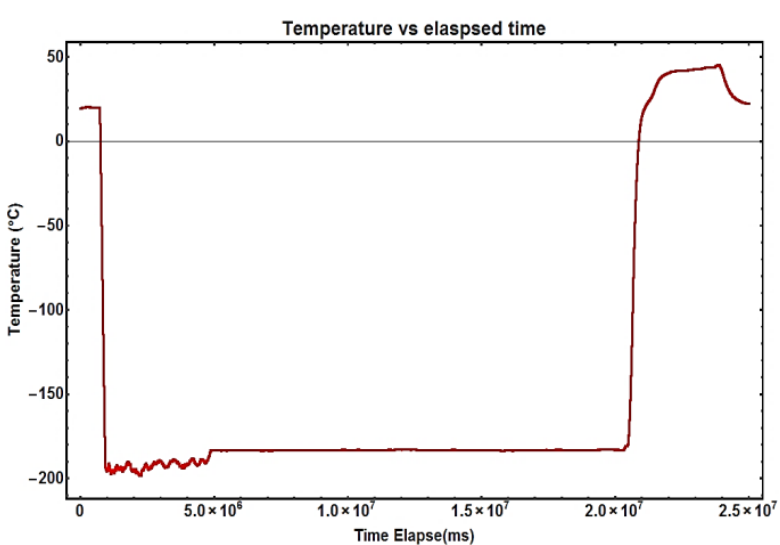

Figure 4-67: Inside Temperature vs Time

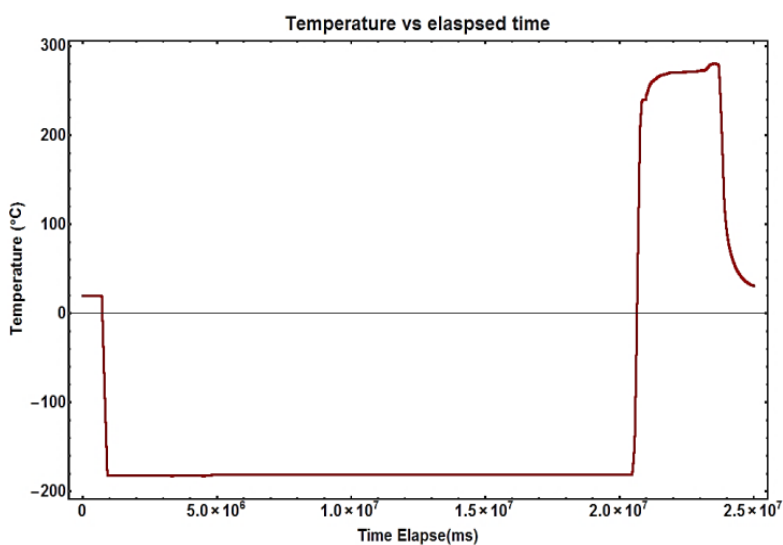

Figure 4-68: Outside Temperature vs Time 


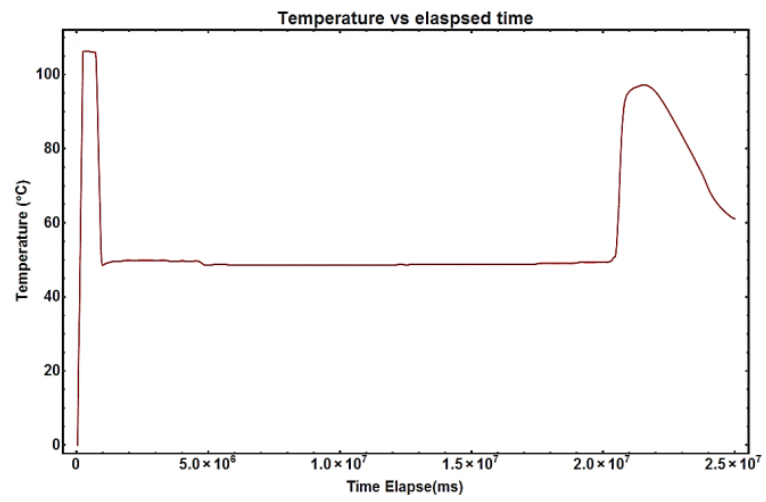

Figure 4-69: Pressure time graph of experimental system.
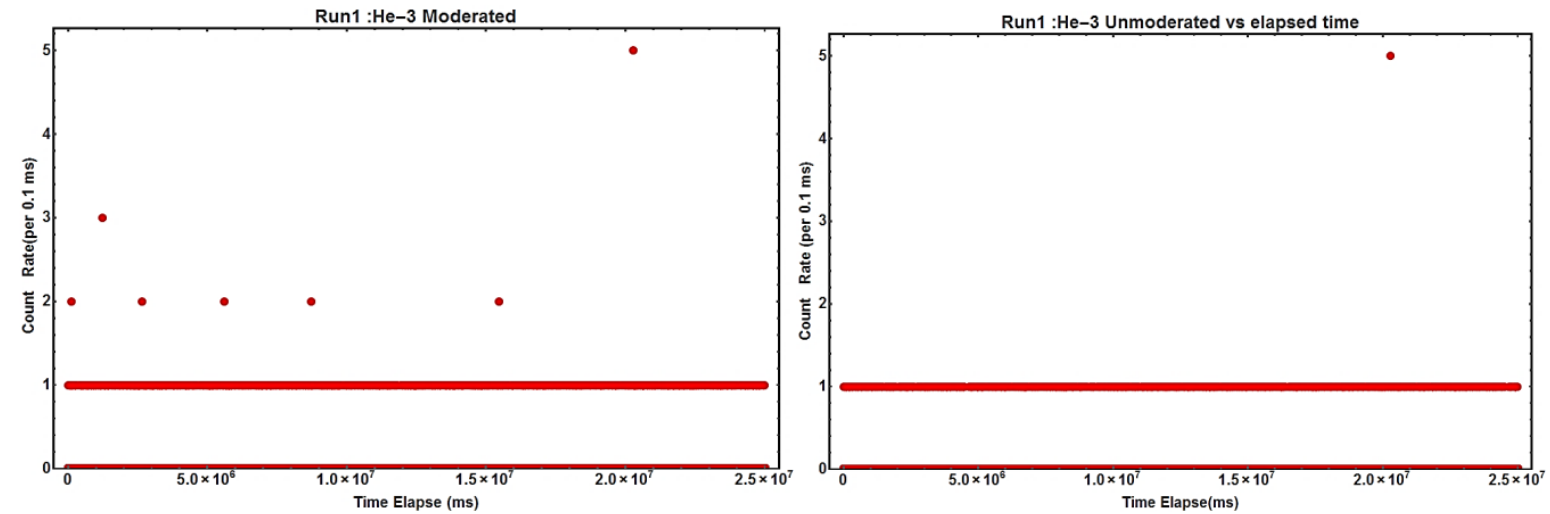

Figure 4-70: Moderated ${ }^{3} \mathrm{He}$ Detector-Foreground Count Count

Figure 4-71: Unmoderated ${ }^{3} \mathrm{He}$ Detector-Foreground

The surface analyses of the titanium samples were done using a scanning electrode microscope. The results are shown in figure 4-76 and figure 4-77. The image taken after the experiment shows cracks were formed after loading the system with titanium. 


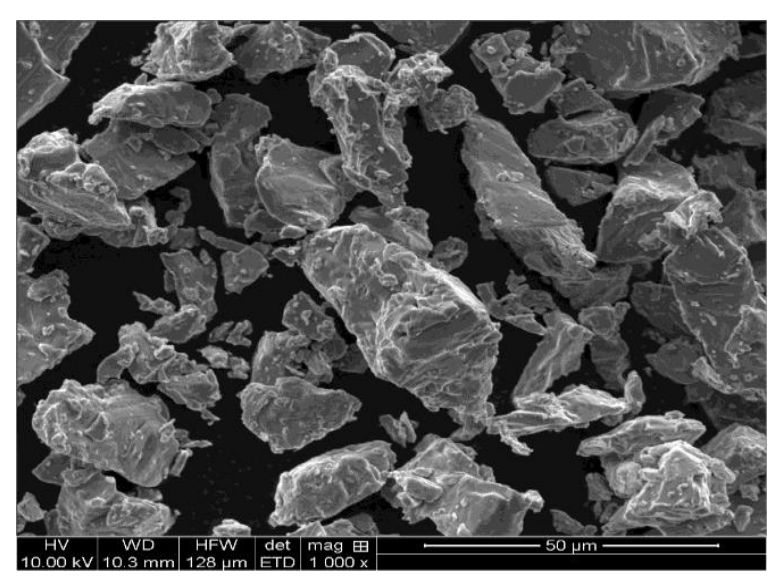

Figure 4-72: SEM Image-Control Sample

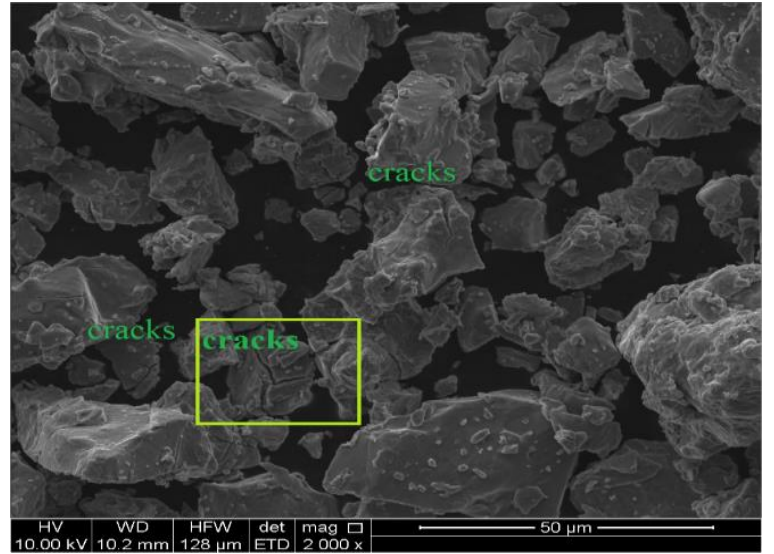

Figure 4-73: SEM Image-Experimental Sample

\section{Experiment 7}

The experimental system was loaded with $12.15 \mathrm{~g}$ of 325 dehydrated titanium mesh $(99.99 \%$ metal basis). The composition of the powder determined by EDS spectroscopy is shown in the figure 4-78 below:

Table 4-7: Composition of Titanium

\begin{tabular}{|l|c|c|c|c|}
\hline $\begin{array}{l}\text { Elemental } \\
\text { Comp. }\end{array}$ & $\begin{array}{c}\text { Atomic } \\
\#\end{array}$ & $\begin{array}{c}\text { Mass } \\
{[\%]}\end{array}$ & $\begin{array}{c}\text { Mass } \\
\text { Norm. [\%] }\end{array}$ & $\begin{array}{c}\text { Abs. } \\
\text { error [\%], } \sigma\end{array}$ \\
\hline Titanium & 22 & 98.80 & 3.49 & 101.05 \\
\hline Carbon & 6 & 1.20 & 0.21 & 1.23 \\
\hline
\end{tabular}

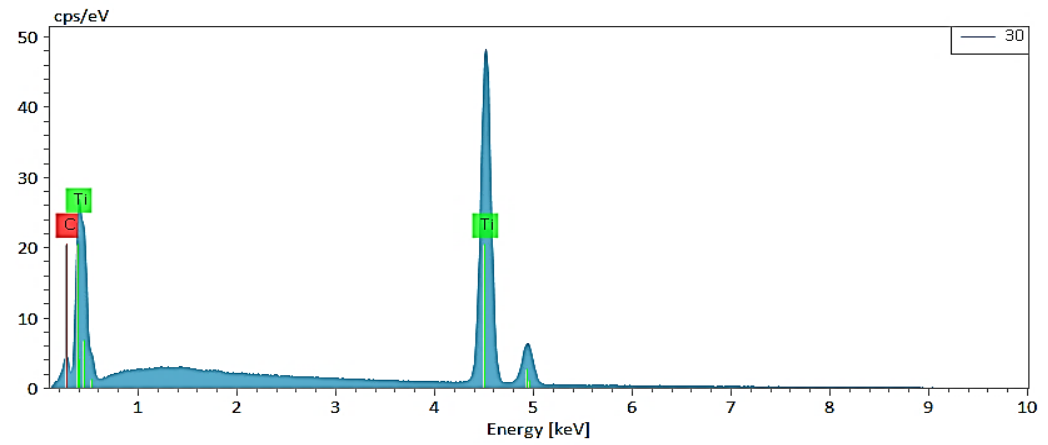

Figure 4-74: Composition of Titanium

The background count rate are shown in figure 4-79 and figure 4-80. The maximum count rate is 2 counts/ $0.001 \mathrm{~ms}$ in the moderated and unmoderated detectors, respectively. 


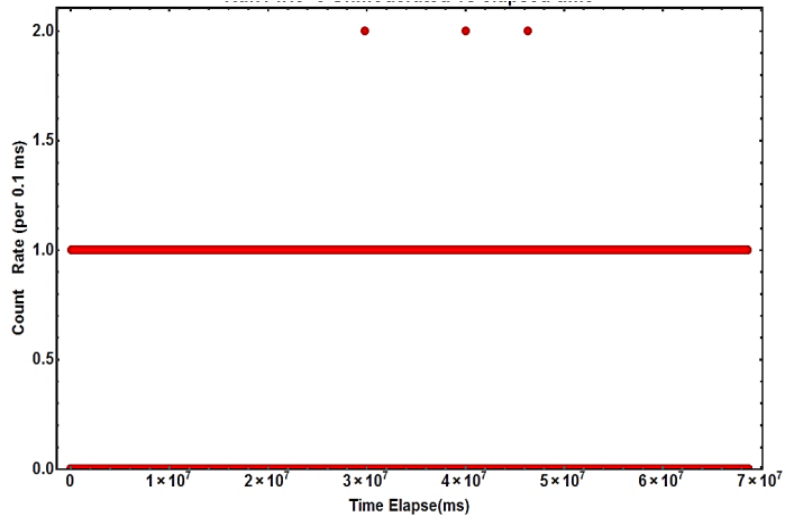

Figure 4-75: Moderated ${ }^{3} \mathrm{He}$ Detector- Background Count

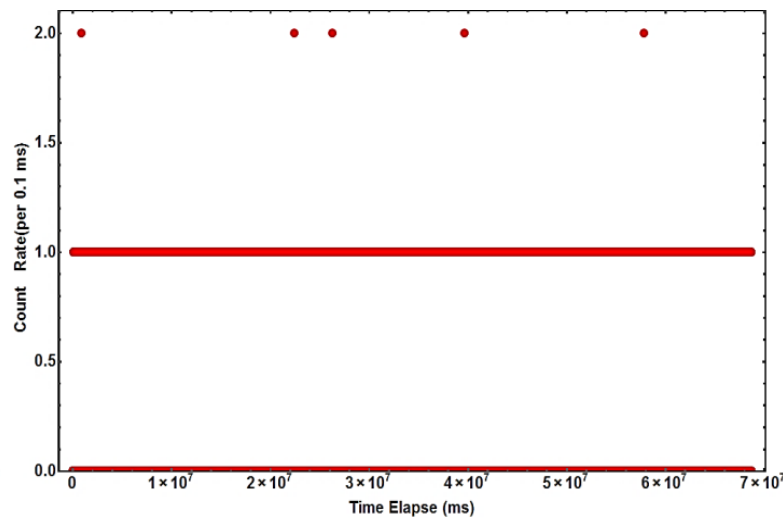

Figure 4-76: Unmoderated ${ }^{3}$ He Detector- Background Count

Figure 4-81 and figure 4-82 shows the temperature data during the experiment. The system was placed in liquid nitrogen for approximately six hours before shocking. Thermal shocking was done using a heat gun. The pressure of the system during and after shocking is shown in figure 4-83 for the entire experiment. No neutron burst was observed during this experiment.

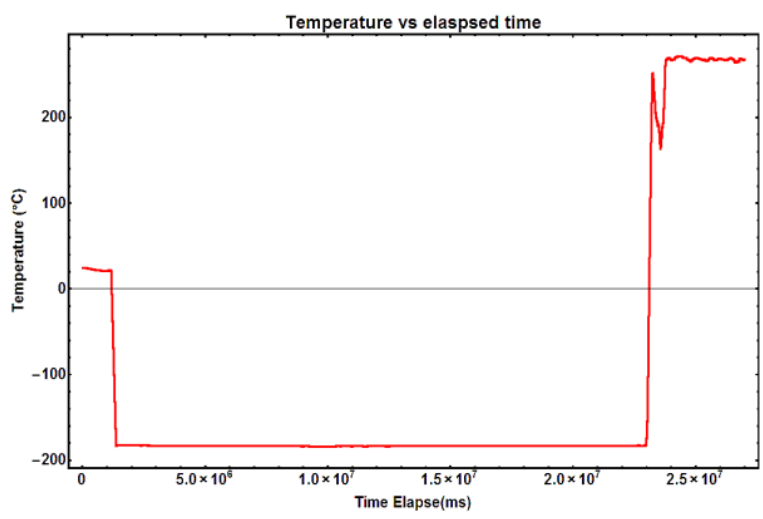

Figure 4-77: Inside Temperature vs Time

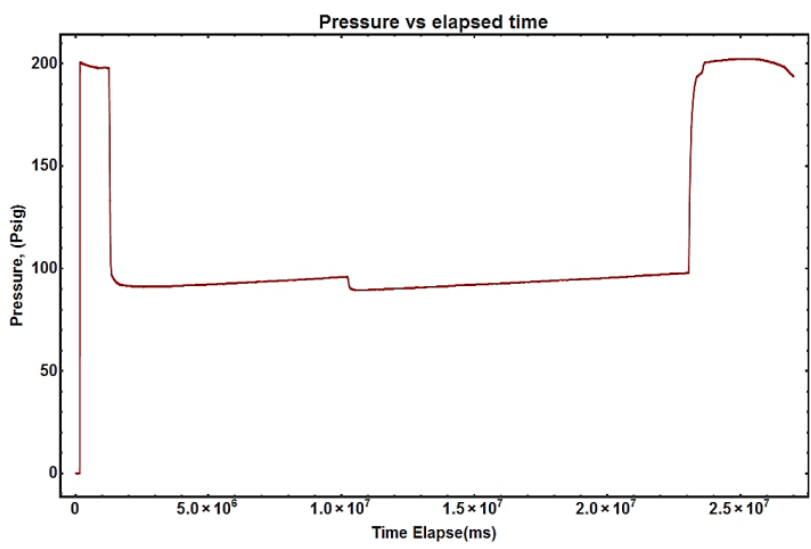

Figure 4-79: Pressure with time

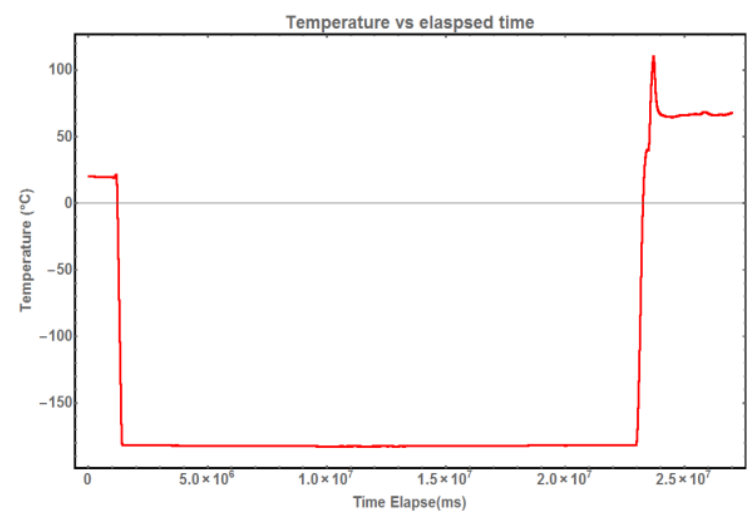

Figure 4-78: Outside Temperature vs Time 


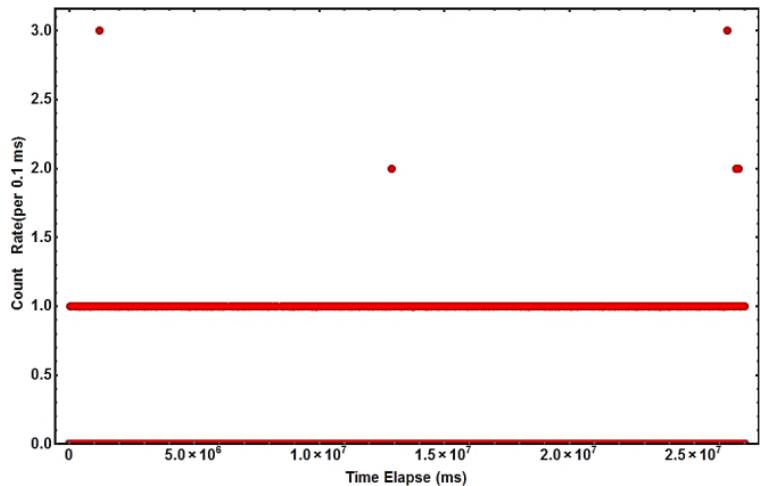

Figure 4-80: Moderated ${ }^{3}$ He Detector- Foreground Count

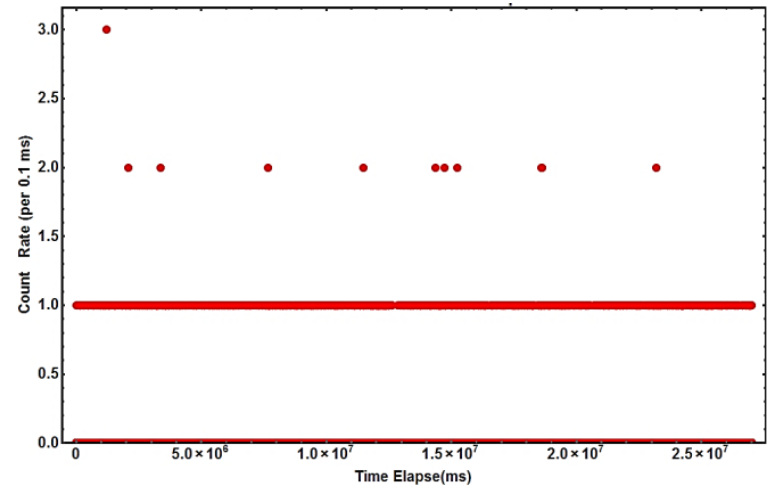

Figure 4-81: Unmoderated ${ }^{3}$ He Detector- Foreground Count

The SEM images are shown in figure 4-86 and figure 4-87. The image of the sample used in the experiment shows cracks produced during loading.

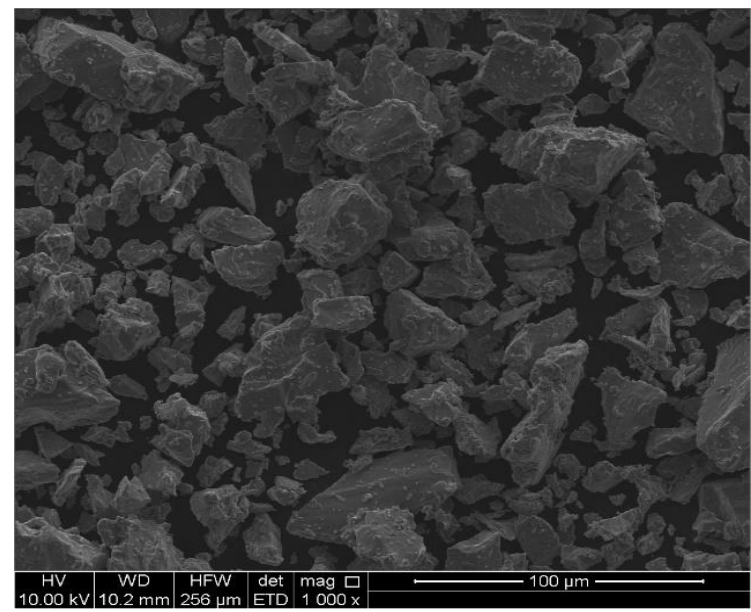

Figure 4-82: SEM images- Control Sample

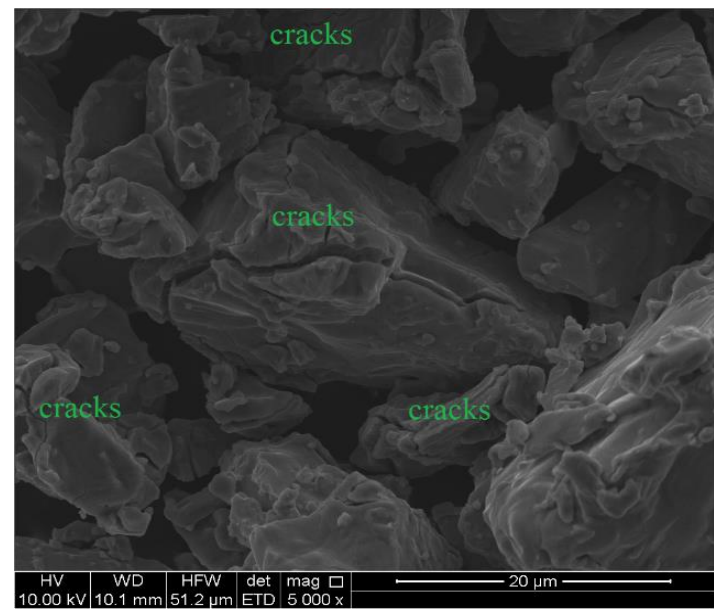

Figure 4-83: SEM images- Experimental Sample

\section{Experiment 8}

The mass of the titanium powder used in this experiment was $13.16 \mathrm{~g}$. The composition of the powder is given below:

Table 4-8: Composition of Titanium

\begin{tabular}{|c|c|c|c|c|}
\hline $\begin{array}{c}\text { Elemental } \\
\text { Comp. }\end{array}$ & $\begin{array}{c}\text { Atomic } \\
\#\end{array}$ & $\begin{array}{c}\text { Mass } \\
{[\%]}\end{array}$ & $\begin{array}{c}\text { Mass } \\
\text { Norm. [\%] }\end{array}$ & $\begin{array}{c}\text { Abs. } \\
\text { error [\%],1 } \sigma\end{array}$ \\
\hline Titanium & 22 & 105.24 & 99.53 & 3.62 \\
\hline Carbon & 6 & 0.47 & 0.47 & 0.10 \\
\hline
\end{tabular}




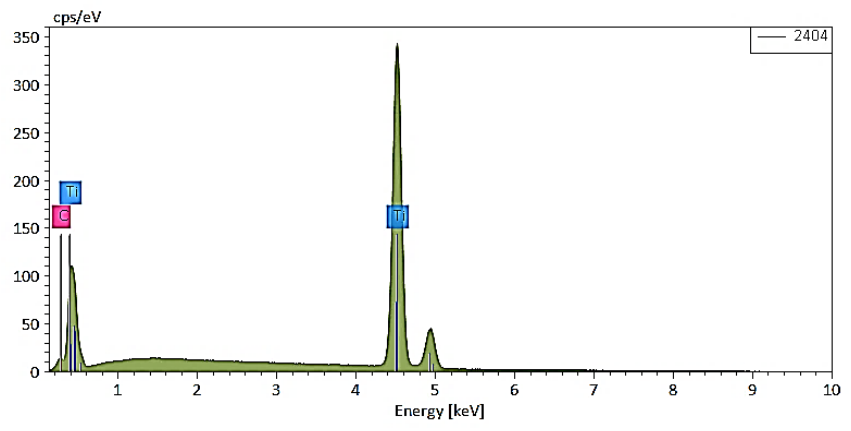

Figure 4-84: Composition of Titanium 8

The background count recorded just before running experiment 8 is shown in figure 4-89 and figure 4-90. The maximum count rate is 2 counts $/ 0.01 \mathrm{~ms}$ in both the moderated and unmoderated helium-3 detectors.

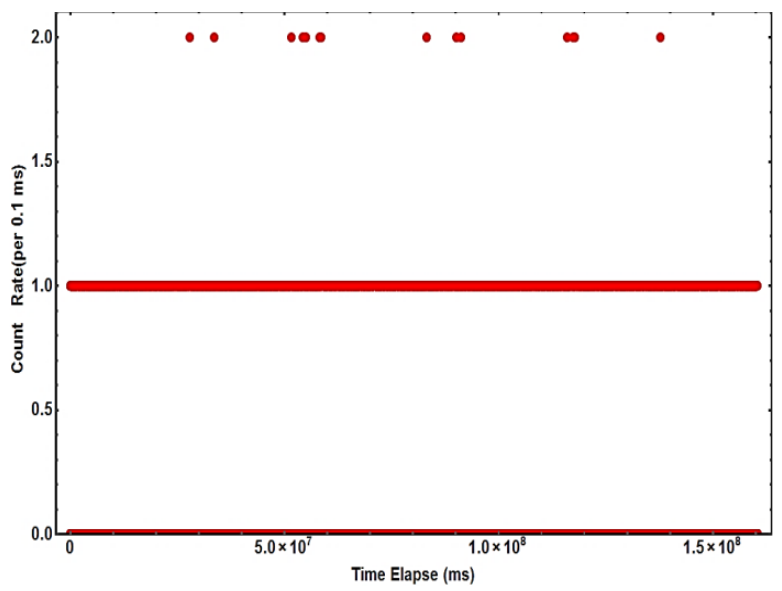

Figure 4-85: Moderated ${ }^{3} \mathrm{He}$ Detector- Background Count

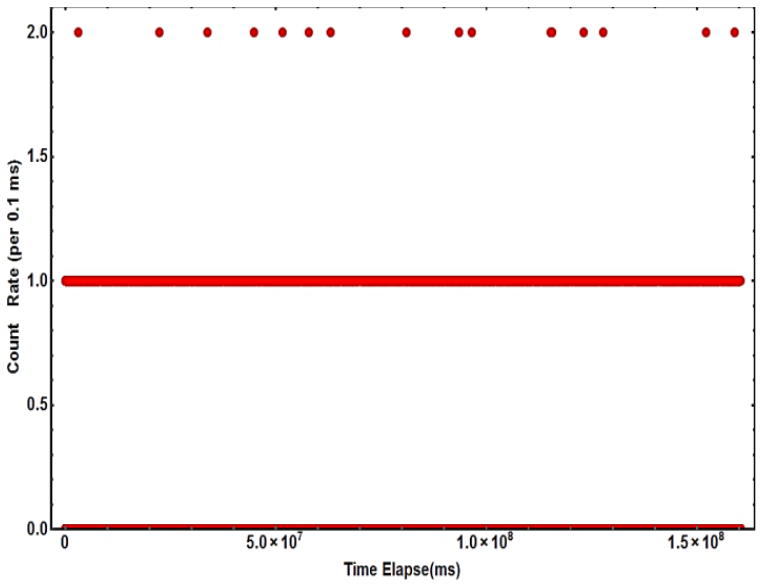

Figure 4-86: Unmoderated ${ }^{3}$ He Detector- Background Count

Figure 4-91, figure 4-92 and figure 4-93 show the temperature against time graphs, and pressure against time graphs of the experimental chamber, before and after shocking, with the maximum temperature of $250^{\circ} \mathrm{C}$ used to achieve the shocking. No neutron burst was observed but the SEM images show cracks were formed when titanium was loaded with titanium. 


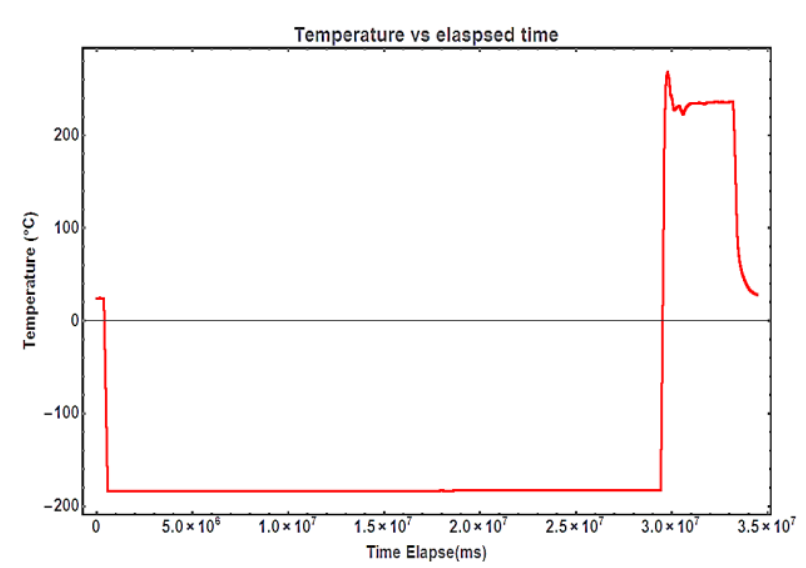

Figure 4-87: Inside Temperature vs Time

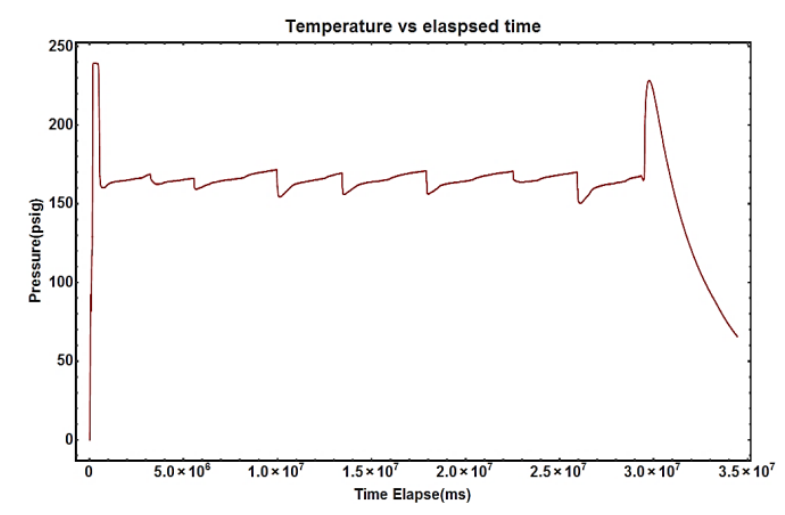

Figure 4-89: Variation of pressure with time
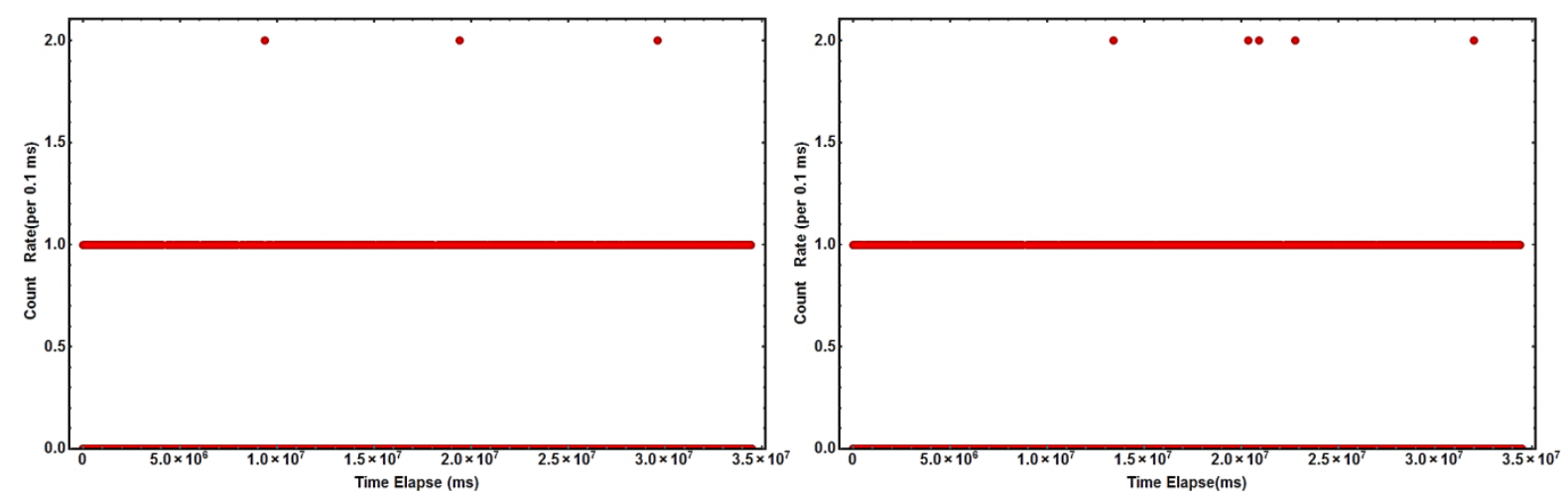

Figure 4-90: Moderated He3 Detector- Foreground Count Figure 4-91: Unmoderated He3 Detector- Foreground Count

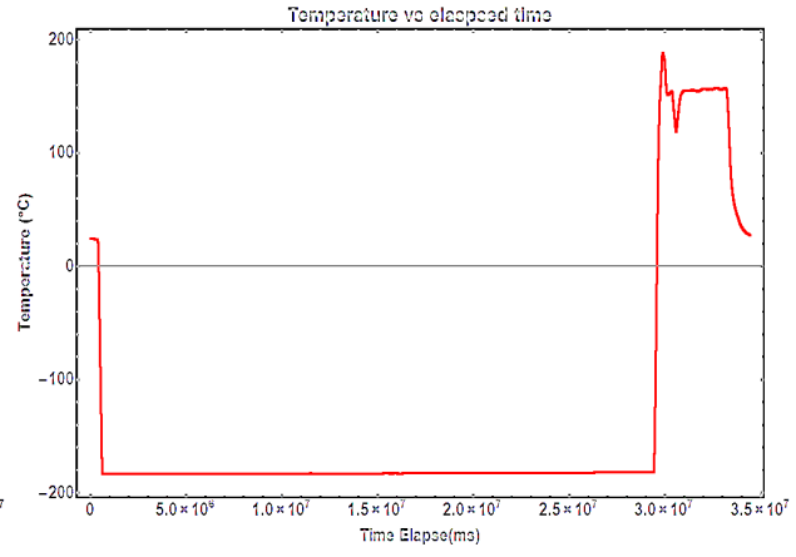

Figure 4-88: Outside Temperature vs Time 


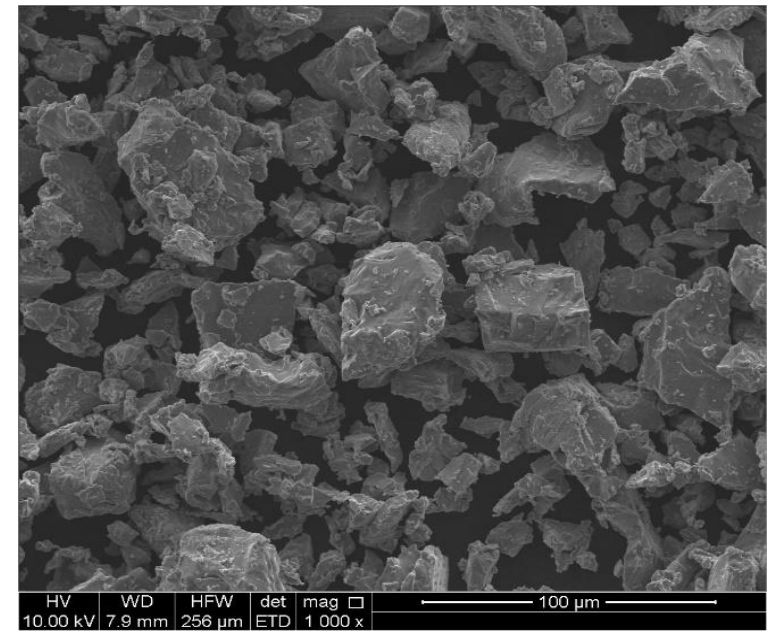

Figure 4-92: SEM Images-Control Sample

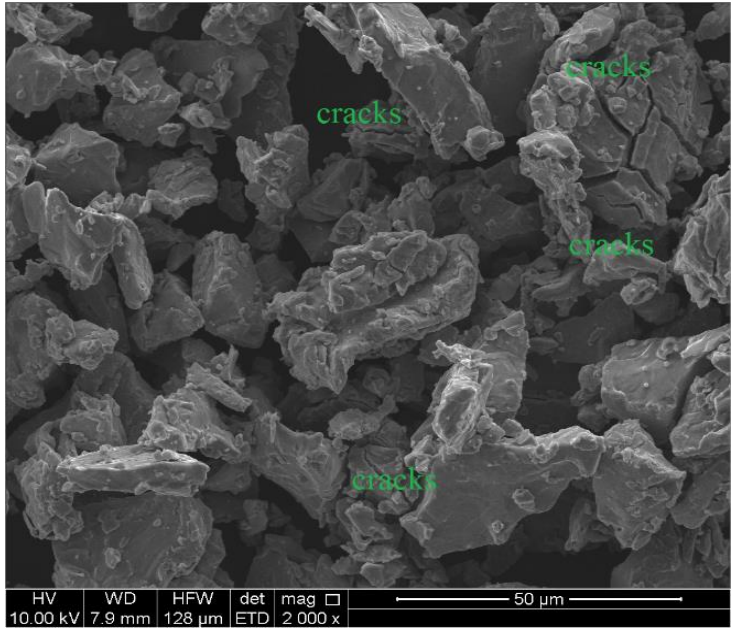

Figure 4-93: SEM Images-Experimental Sample

\section{Experiment 9}

The mass of titanium used in this experiment was $7.01 \mathrm{~g}$. The composition of the powder was determined using the EDS spectroscopy techniques. The results are shown below:

Table 4-9: Composition of Titanium

\begin{tabular}{|c|c|c|c|c|}
\hline $\begin{array}{c}\text { Elemental } \\
\text { Comp. }\end{array}$ & $\begin{array}{c}\text { Atomic } \\
\#\end{array}$ & $\begin{array}{c}\text { Mass } \\
{[\%]}\end{array}$ & $\begin{array}{c}\text { Mass } \\
\text { Norm. } \\
{[\%]}\end{array}$ & $\begin{array}{c}\text { Abs. } \\
\text { error [\%], } \sigma\end{array}$ \\
\hline Titanium & 22 & 105.24 & 99.53 & 3.62 \\
\hline Carbon & 6 & 0.47 & 0.47 & 0.10 \\
\hline
\end{tabular}

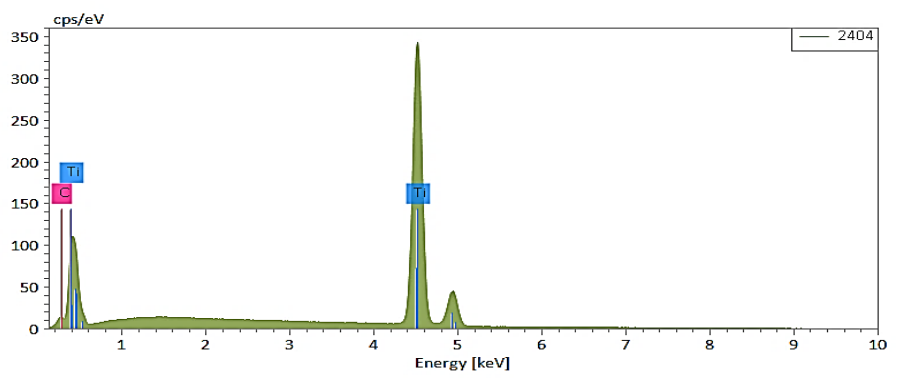

Figure 4-94: Composition of Titanium

The observed background count rate before running the experiment is shown in figure 4-99 and figure 4-100. The maximum count rate is 2 counts/ $0.01 \mathrm{~ms}$ and the average count rates of 
0.004505 and 0.00412 counts/s were recorded in the moderated and unmoderated helium-3 detectors, respectively.
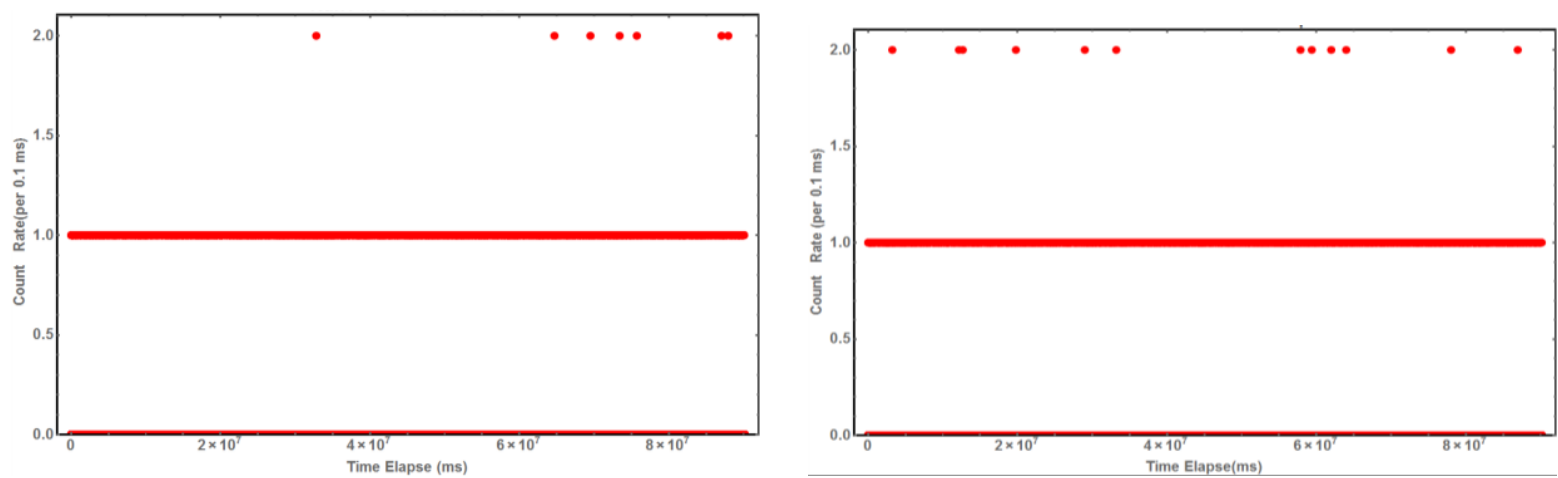

Figure 4-95:Moderated ${ }^{3} \mathrm{He}$ Detector- Background Count Figure 4-96: Unmoderated ${ }^{3} \mathrm{He}$ Detector-Background Count

Two trials were conducted in the experiment. In the first trial, the system was shocked in boiling water while in the second trial, the system was pumped-down with a diffusion pump, and deuterium was later introduced into titanium and after placing the system in liquid nitrogen for more than 6 hours, shocking was achieved using a heat gun. The temperature and pressure data for trial 1 is shown in figure 4-101 - 4-103. The foreground count rate during trial 1 is shown in figure 4-105-4-106. No neutron burst was observed during trial 1and trial 2.

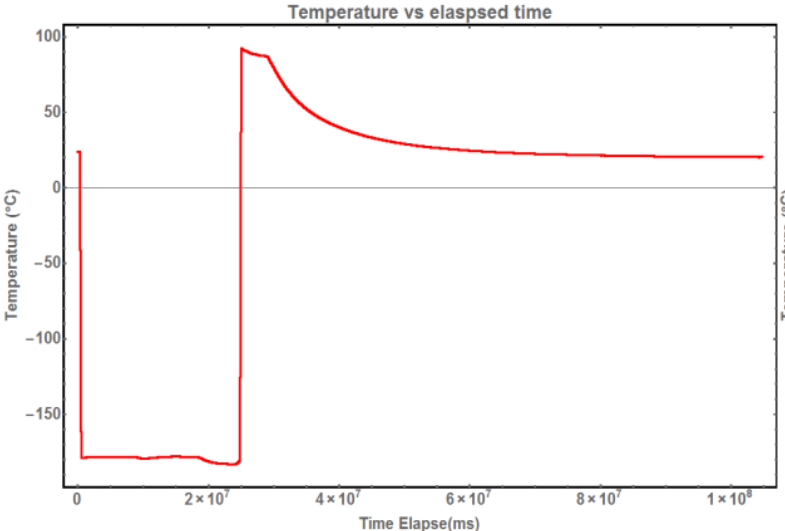

Figure 4-97: Inside Temperature with Time Trial 1

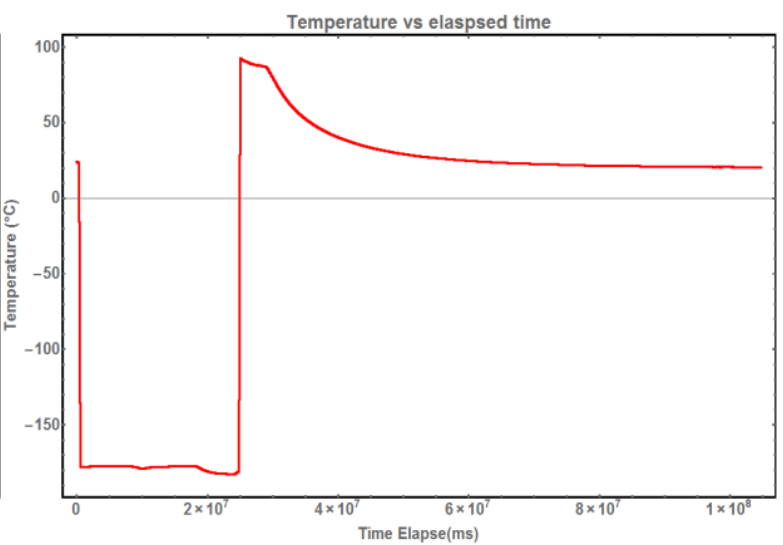

Figure 4-98: Outside Temperature with Time Trial 2 


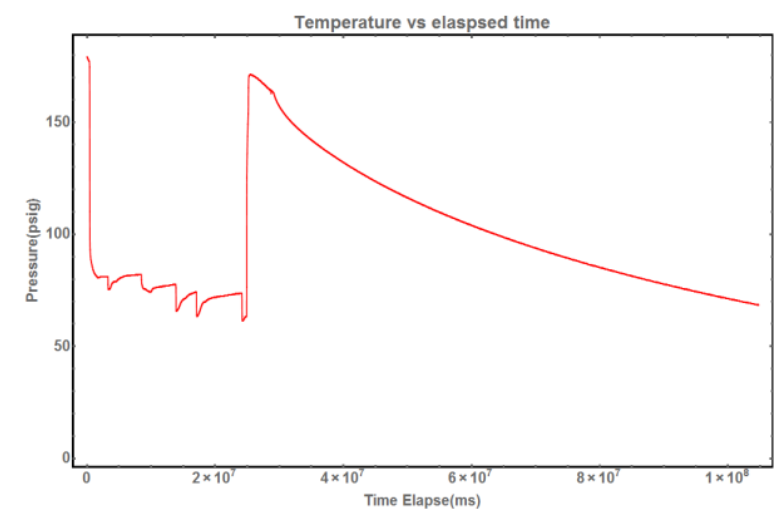

Figure 4-99: Variation of Pressure vs time Trial 1
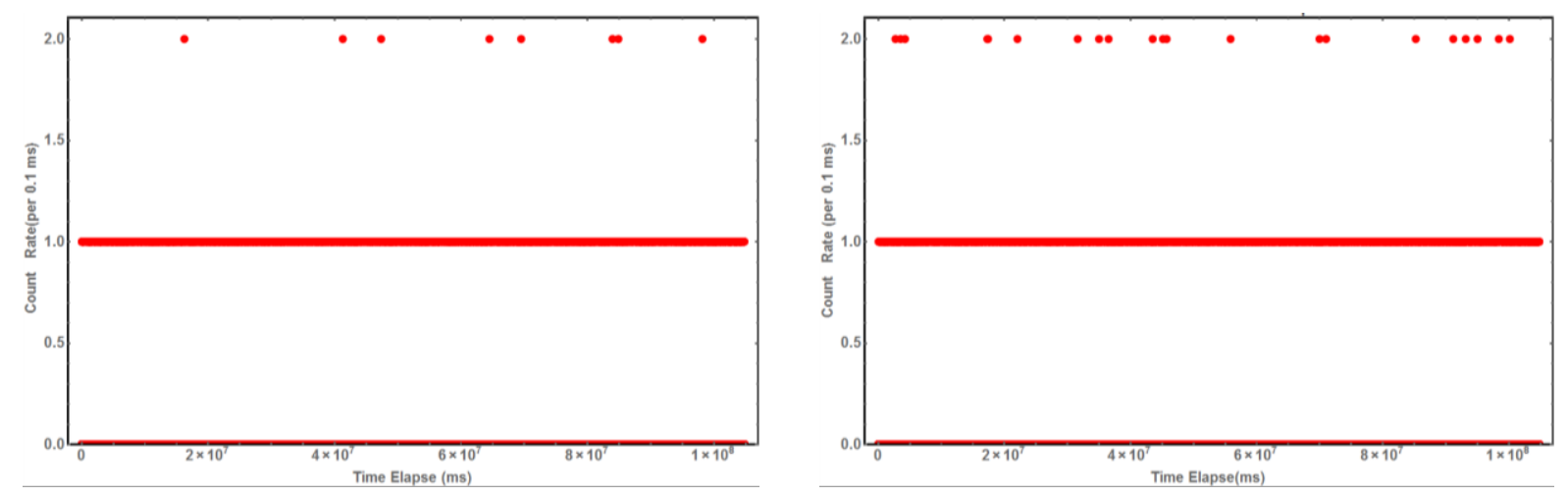

Figure 4-100: Moderated ${ }^{3}$ He Detector- Foreground Count $\quad$ Figure 4-101: Moderated ${ }^{3} \mathrm{He}$ - Foreground Count
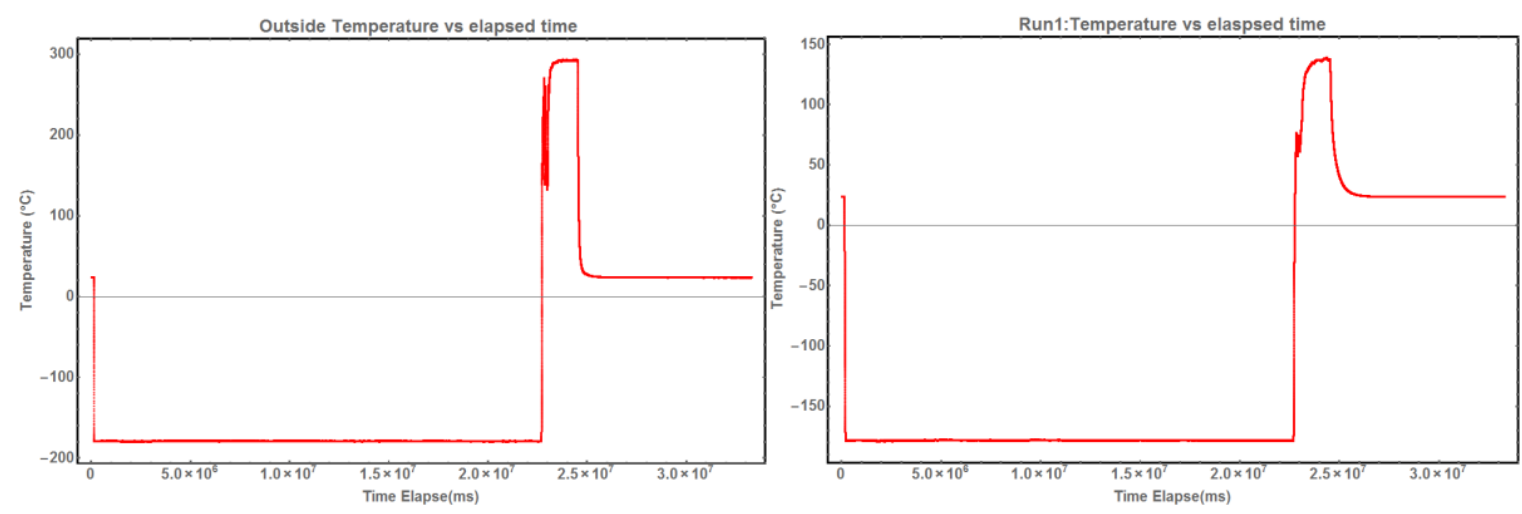

Figure 4-102: Temperature change-first trial outside(right) (Inside left) Trial 2 


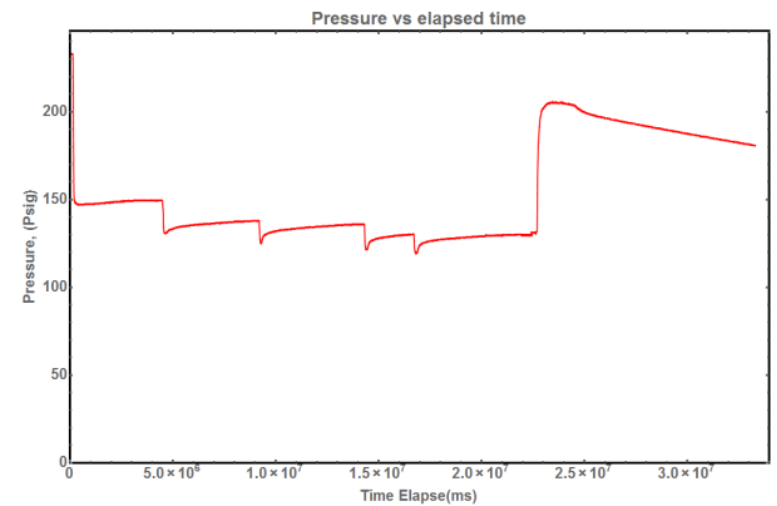

Figure 4-103: Pressure variation with time Trial 2
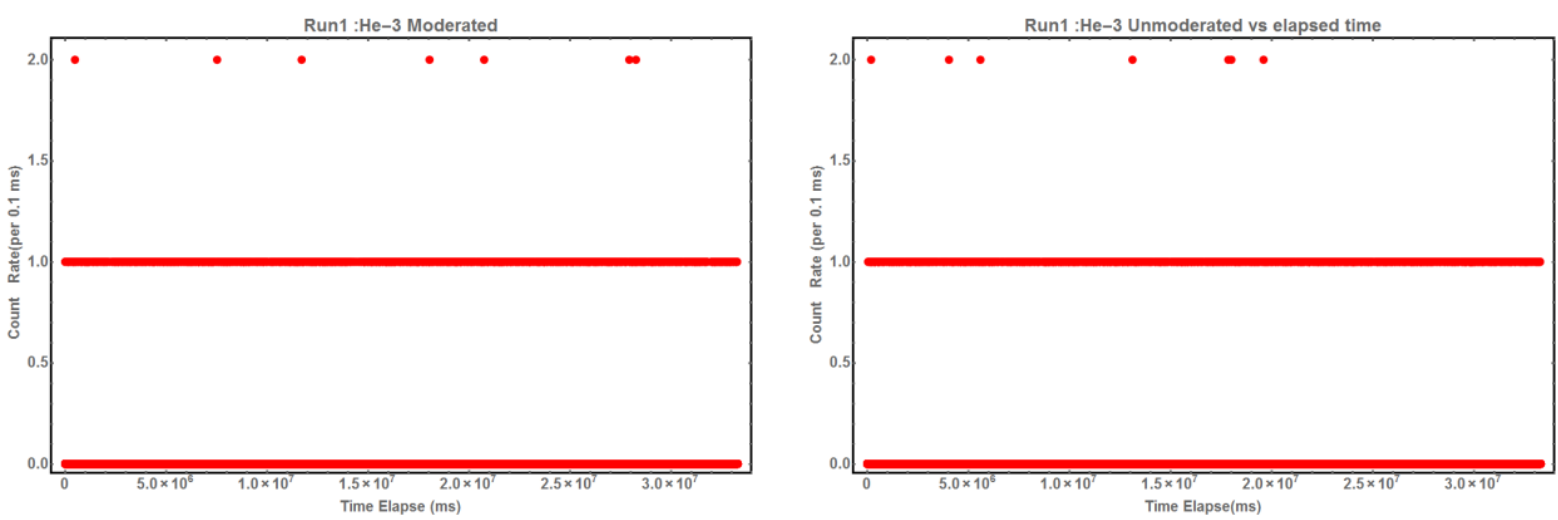

Figure 4-104: Moderated ${ }^{3}$ He Detector- Foreground Count Figure 4-105: Unmoderated ${ }^{3}$ He Detector- Foreground Count

The surface morphology of the titanium sample is shown in figure 4-110 and figure 4-111.

No cracks were produced during deuterium loading after the two experimental trials.

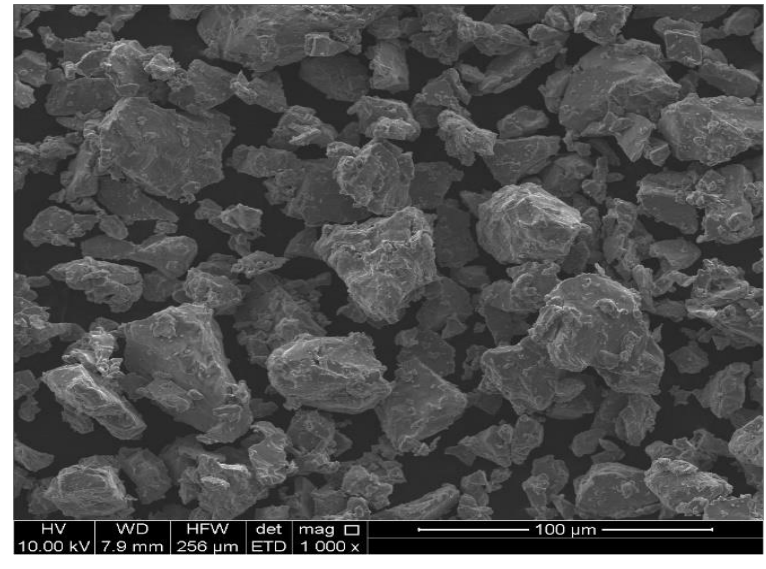

Figure 4-106: SEM Image-Control Sample

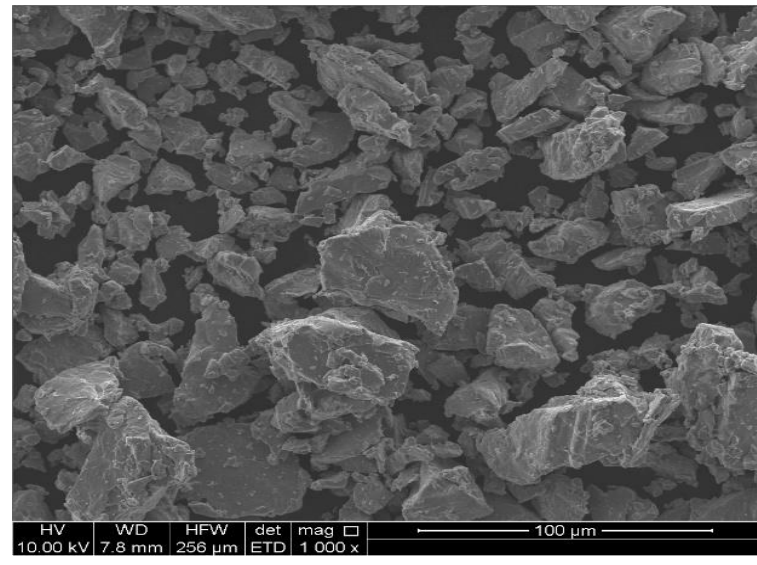

Figure 4-107: SEM Image -Experimental Sample 


\subsubsection{Liquid Scintillation Detector Analysis}

The liquid scintillation counter (LSC) detector was used to count the titanium samples for the presence of tritium. The LSC calibration was performed using tritium, a low energy beta, carbon-14, a medium energy and Am-241, an alpha emitter. The higher beta radiation produces a higher energy efficiency in LSC. The LSC efficiency depends on the degree of quenching, the nature of the sample used, the type of scintillator, and the preparation method. The LSCS has $56 \%$ efficiency when counting with ${ }^{3} \mathrm{H}$ and $75 \%$ efficiency with ${ }^{14} \mathrm{C}$. Fiveminute counts were collected in all the samples. The minimum detectable limit for tritium beta using this liquid scintillation is $5.09 \times 10^{-6} \mu C i(11.3 \mathrm{dpm})$. This corresponds to about $4.85 \times 10^{-10}$ atoms of tritium. Thus, any tritium content less than this amount will not be detected.

Table 4-10: Liquid Scintillation Calibration Data

\begin{tabular}{|c|c|c|c|c|c|}
\hline Isotope & Efficiency & MDA(DPM) & BKG(CPM) & MDA( $\boldsymbol{\mu C i})$ & CPM=DPM \\
\hline $\mathrm{H}-3$ & $56 \%$ & 11.3 & 8 & $5.09 \times 10^{-6}$ & $112=200$ \\
\hline C-14/S35/P-33 & $75 \% \Delta$ & 9.6 & 11 & $4.31 \times 10^{-6}$ & $150=200$ \\
\hline P-32 (Si-32) & $78 \% \Delta$ & 9.6 & 12 & $4.33 \times 10^{-6}$ & $156=200$ \\
\hline Am-241 & $100 \% *$ & 3.2 & 1 & $1.45 \times 10^{-6}$ & $200=200$ \\
\hline Cl-36 & $98 \% *$ & 11.7 & 33 & $5.26 \times 10^{-6}$ & $196=200$ \\
\hline
\end{tabular}

Protocol for Surveys:

$\Delta$ Standard Swipe Test

*Standard Alpha/Beta

The foreground count rates recorded from all the samples used in this work are shown in table 14.11. The LSC allows the user to select keV regions of interest. For this work, three channels selected were: Channel A, 0.0 - $18.6 \mathrm{keV}$, Channel B 18.6 - $256 \mathrm{keV}$, and Channel C 256- $2000 \mathrm{keV}$. Channel A represents the entire energy region of ${ }^{3} \mathrm{H}$. Channel B represents the region from the top of Channel $\mathrm{A}$ to the maximum possible energy of ${ }^{14} \mathrm{C}, 156 \mathrm{keV}$, and Channel C is the entire region (up to $2000 \mathrm{keV}$ ). The Spectra Index of the External Standard 
(Tsie) is also shown in this Table. Tsie is used to determine the counting efficiency. Since high energy betas are not affected by quenching as are low energy beta, hence the effect of quenching will be larger in the counting region $2-18.6 \mathrm{keV}$ than in other regions. In the case where the energy of the radiation source is larger than $18.6 \mathrm{keV}$, the quench can easily shift some of the counts to the lower channel. In our case, the expected beta energy is from tritium which has maximum beta energy of $18.6 \mathrm{keV}$. Hence, all the counts are expected to be registered in Channel A or Channel B. The conversion from CPM to DPM requires knowledge of the efficiency for each sample at its quench level from the calibration curve. The counter had been programmed to calculate the DPM.

The background counts are given below in the three regions:

Table 4-11: Summary of Background count rate of LSC

\begin{tabular}{|c|c|c|c|}
\hline Counts & $\begin{array}{c}\text { Channel A } \\
\mathbf{2 . 0}-\mathbf{1 8 . 6} \mathbf{~ k e V}\end{array}$ & $\begin{array}{c}\text { Channel B } \\
\mathbf{1 8 . 6}-\mathbf{2 5 6} \mathbf{~ k e V}\end{array}$ & $\begin{array}{c}\text { Channel C } \\
\mathbf{2 5 6 . 0}-\mathbf{2 0 0 0} \mathbf{~ k e V}\end{array}$ \\
\hline $\begin{array}{c}\text { Bkg. Subtract } \\
\text { (CPM) }\end{array}$ & 11.79 & 10.59 & 12.53 \\
\hline
\end{tabular}

Table 4-12: Summary of LSC results from Titanium-deuterium loading

\begin{tabular}{|c|c|c|c|c|c|c|c|c|}
\hline $\begin{array}{c}\text { Sample } \\
\text { Number }\end{array}$ & tSIE & \multicolumn{2}{|c|}{$\begin{array}{c}\text { Counting } \\
\text { Region } \\
\text { 2-18.6 KeV }\end{array}$} & \multicolumn{2}{c|}{$\begin{array}{c}\text { Counting } \\
\text { Region } \\
\text { 18.6-256 KeV }\end{array}$} & $\begin{array}{c}\text { Counting } \\
\text { Region } \\
\text { 256-2000 KeV }\end{array}$ & Total \\
\hline $\begin{array}{c}\text { Sample 1 } \\
\text { Control }\end{array}$ & 355.88 & 0 & 0 & 4 & 6 & 2 & 2 & 8 \\
\hline $\begin{array}{c}\text { Sample 1 } \\
\text { Experimental }\end{array}$ & 440.79 & 2 & 3 & 2 & 3 & 3 & 4 & 10 \\
\hline $\begin{array}{c}\text { Sample 2 } \\
\text { Control }\end{array}$ & 382.51 & 0 & 1 & 4 & 5 & 0 & 1 & 6 \\
\hline $\begin{array}{c}\text { Sample 2 } \\
\text { Experimental }\end{array}$ & 415.72 & 0 & 0 & 0 & 0 & 0 & 0 & 0 \\
\hline $\begin{array}{c}\text { Sample 3 } \\
\text { Control }\end{array}$ & 388.95 & 0 & 0 & 0 & 1 & 0 & 0 & 1 \\
\hline $\begin{array}{c}\text { Sample 3 } \\
\text { Experimental }\end{array}$ & 358.93 & 0 & 0 & 4 & 5 & 1 & 1 & 7 \\
\hline
\end{tabular}




\begin{tabular}{|c|c|c|c|c|c|c|c|c|}
\hline $\begin{array}{c}\text { Sample } 4 \\
\text { Control }\end{array}$ & 355.88 & 0 & 0 & 4 & 6 & 2 & 2 & 8 \\
\hline $\begin{array}{c}\text { Sample } 4 \\
\text { Experimental }\end{array}$ & 395.68 & 0 & 0 & 2 & 2 & 3 & 4 & 6 \\
\hline $\begin{array}{c}\text { Sample } 5 \\
\text { Control }\end{array}$ & 291.70 & 0 & 0 & 3 & 4 & 0 & 0 & 4 \\
\hline $\begin{array}{c}\text { Sample 5 } \\
\text { Experimental }\end{array}$ & 183.27 & 0 & 0 & 0 & 0 & 0 & 1 & 1 \\
\hline $\begin{array}{l}\text { Sample } 6 \\
\text { Control }\end{array}$ & 418.39 & 0 & 0 & 1 & 2 & 1 & 1 & 3 \\
\hline $\begin{array}{c}\text { Sample 6 } \\
\text { Experimental }\end{array}$ & 395.68 & 0 & 0 & 5 & 7 & 2 & 2 & 9 \\
\hline $\begin{array}{c}\text { Sample } 7 \\
\text { Control }\end{array}$ & 390.43 & 0 & 0 & 1 & 1 & 1 & 2 & 3 \\
\hline $\begin{array}{c}\text { Sample } 7 \\
\text { Experimental }\end{array}$ & 396.14 & 0 & 0 & 4 & 5 & 0 & 0 & 5 \\
\hline $\begin{array}{c}\text { Control of } \\
\text { Experiment } \\
8 \\
\end{array}$ & 215.04 & 0 & 0 & 2 & 3 & 4 & 5 & 8 \\
\hline $\begin{array}{c}\text { Experimental } \\
\text { of } \\
\text { Experiment } \\
8\end{array}$ & 312.26 & 0 & 0 & 6 & 7 & 0 & 0 & 8 \\
\hline $\begin{array}{c}\text { Control of } \\
\text { Experiment } \\
9\end{array}$ & 223.73 & 0 & 0 & 3 & 5 & 1 & 1 & 6 \\
\hline $\begin{array}{c}\text { Experimental } \\
\text { of } \\
\text { Experiment } \\
9\end{array}$ & 371.26 & 0 & 0 & 0 & 0 & 1 & 1 & 1 \\
\hline
\end{tabular}

The maximum total activity obtained with the $\operatorname{LSC}$ is $10 \mathrm{dpm}\left(4.5 \times 10^{-6} \mu \mathrm{Ci}\right)$ obtained from sample 1 , which is lower than the minimum detectable limit $\left(5.09 \times 10^{-6}\right.$ $\mu C i$ ) of this instrument and, also lower than the background counts. This is an indication that tritium is absent in all these samples. 


\section{REFERENCES}

1. Srivastava, Y., A. Widom, and L. Larsen, A primer for electroweak induced lowenergy nuclear reactions. Low Energy Nuclear Reactions and New Energy Technologies Sourcebook, ed. S. Krivit and J. Marwan, American Chemical Society, Oxford University Press, Washington, DC, 2010. 2.

2. Widom, A. and L. Larsen, Theoretical standard model rates of proton to neutron conversions near metallic hydride surfaces. arXiv preprint nucl-th/0608059, 2006.

3. Widom, A. and L. Larsen, Ultra low momentum neutron catalyzed nuclear reactions on metallic hydride surfaces. The European Physical Journal C-Particles and Fields, 2006. 46(1): p. 107-111.

4. $\quad$ Pelowitz, D.B., et al., MCNPX 2.7 E extensions. 2011, Los Alamos National Laboratory (LANL). 


\section{CONCLUSION AND FUTURE WORK}

The investigation of neutron detection from deuterium-titanium systems is important because it can provide a viable neutron source for calibration of neutron detectors and nondestructive analysis. The mechanism through which neutrons are produced in these systems is not well understood and experimental results are often irreproducible. Neutrons are postulated to be produced during the warm up phases of the titanium-deuterium system due to non-equilibrium conditions resulting from change in temperature and pressure of the system during absorption or desorption phases of deuterium in titanium. Another mechanism, known as fracto-fusion mechanism has been proposed to explain the means through which nuclear emissions results from condense matter. The fracto-fusion hypothesis suggests that a nuclear effect occurs from fracture caused by mechanical stress in crystals lattice. Cracks could result from internal pressure, or temperature variations, or both in solid matter. The formation of cracks in crystals creates traps that can hold huge amount of deuterium within the crystal structure of solids for titanium-deuterium interaction to occur leading to neutron emissions. This work was geared at understanding the reason for the inconsistency in neutron emissions from titanium-deuterium systems by investigating the roles of phase transitions, crack formation, heat production, the ratio atoms of titanium with respect to deuterium, and the surface treatment of titanium sample in neutron production. Three detectors were used in this investigation: a moderated helium-3 detector, an unmoderated helium-3 detector and a proton recoil detector. The detectors were calibrated using a Cf-252, and a PuBe source. The investigation involved using dehydrided, -325 titanium mesh with diameter of $14 \mu \mathrm{m}$ loaded with deuterium and subjecting the system to non-equilibrium conditions by repeatedly placing in liquid nitrogen followed by rapid warm up phases. The results show that degassing the system under high vacuum, while baking the system at high temperature, increases deuterium absorption in titanium lattice. The degassing procedure prevents the formation of 
oxide layers on the surface of titanium which inhibits deuterium absorption are easily removed at high temperatures, ensuring that deuterium atoms are inserted in titanium lattice. The presence of impurities in this system limits dehydriding. It is recommended that these experiments be carried out under high vacuum conditions. The X-ray diffraction pattern shows that titanium hydride is formed during deuterium loading. The loading of titanium with deuterium in titanium leads to a phase change from $\alpha$-titanium to $\delta$-titanium at room temperature but no noticeable neutron emission was observed during this phase change. Phase changes in titanium crystal leads to modifications in the lattice structure of titanium and increases its volume and size. The phase transition that occurs during titanium deuteride formation is exothermic leading to the release of heat. A large temperature increase was observed in two experiments during phase transition. The increase in temperature reduces the diffusion time, thus increasing the probability of titanium-deuterium reaction occurring. Cracks were observed in several titanium samples after loading with deuterium. Deuterium absorption process occurred much more rapidly in samples where cracks were formed. The cracks were also produced in certain locations in the sample and not in others. Hence, should a neutrons emission occurred, the nuclear reaction will occur at this location. However, neutrons burst was not observed in samples with large cracks. The observed neutrons produced from titanium-deuterium system were very small and only single neutron burst events were observed in an entire experiment. The occurrence of neutrons occurred in two of the 9 experiments conducted. The samples were analyzed for tritium production using a liquid scintillation detector, but tritium was not observed in any of the samples. There was also no evidence of transmutation occurring in this samples. We hypothesized that the titanium-deuterium reaction is a low probability process that is influence by crack formation. The process is likely due to a statistical process that depends on sample microstructure, 
number of defects, preparation condition and shocking procedure. It is recommended that in $\alpha$-ttitanium. 


\section{Appendix A}

Appendix A shows the composition of titanium samples used in the experiment. The regions where the analysis was performed is shown in Appendix B.

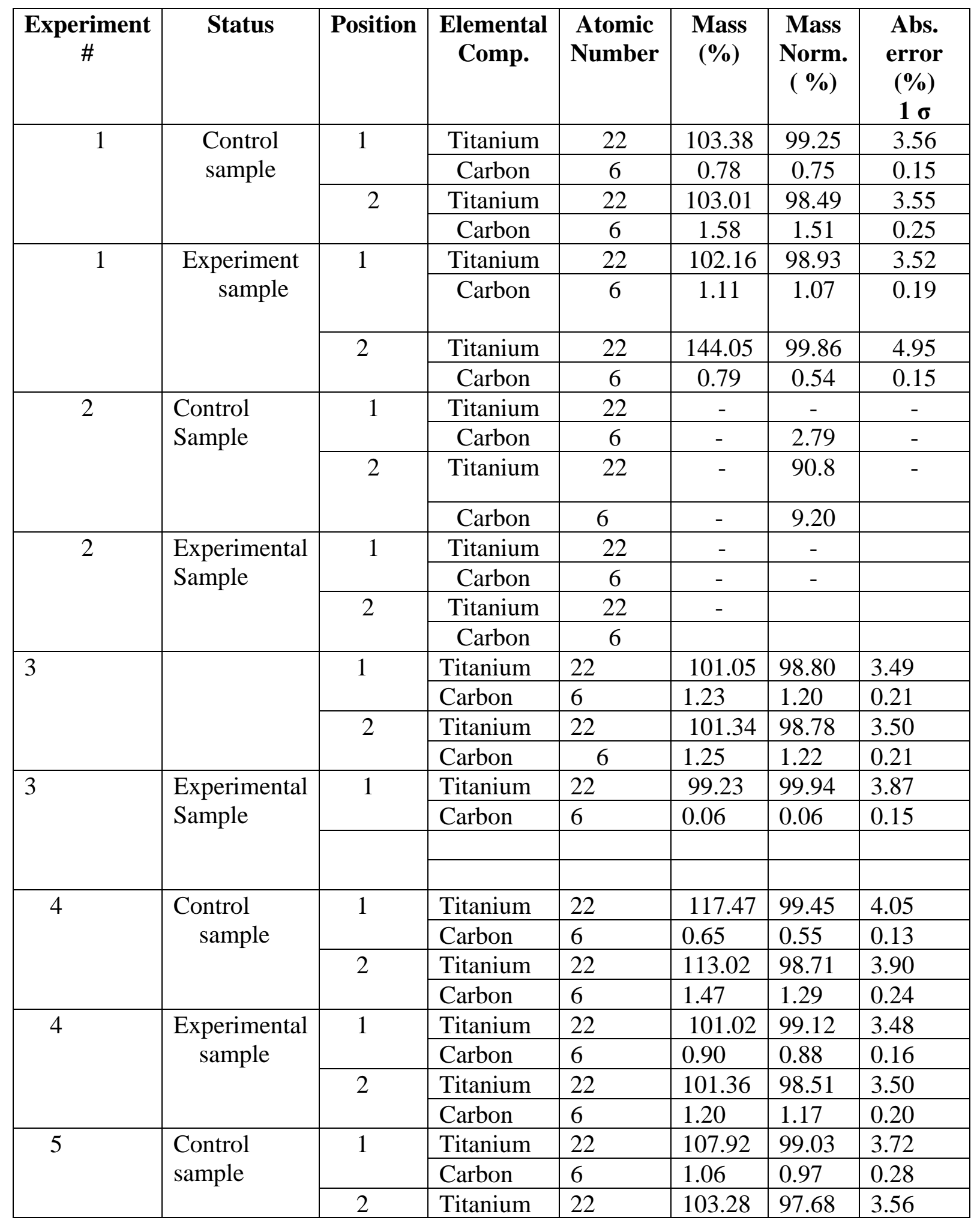




\begin{tabular}{|c|c|c|c|c|c|c|c|}
\hline & & & Carbon & 6 & 2.23 & 2.11 & 0.32 \\
\hline \multirow{4}{*}{5} & \multirow{4}{*}{$\begin{array}{l}\text { Experiment } \\
\text { sample }\end{array}$} & \multirow[b]{2}{*}{1} & Silicon & 14 & 0.22 & 0.21 & 0.04 \\
\hline & & & Titanium & 22 & 133.85 & 99.19 & 4.61 \\
\hline & & \multirow[b]{2}{*}{2} & Carbon & 6 & 1.09 & 0.81 & 0.19 \\
\hline & & & Titanium & 22 & 96.78 & 97.21 & 3.34 \\
\hline \multirow[t]{4}{*}{6} & & & Titanium & 22 & 134.14 & 99.43 & 4.62 \\
\hline & & & Carbon & 6 & 0.77 & 0.57 & 0.15 \\
\hline & & 2 & Titanium & 22 & 100.87 & 98.84 & 3.48 \\
\hline & & & Carbon & 6 & 1.18 & 1.16 & 0.20 \\
\hline \multirow[t]{4}{*}{6} & \multirow{4}{*}{$\begin{array}{l}\text { Experiment } \\
\text { sample }\end{array}$} & \multirow[t]{2}{*}{1} & Titanium & 22 & 102.03 & 99.18 & 3.52 \\
\hline & & & Carbon & 6 & 0.85 & 0.82 & 0.16 \\
\hline & & \multirow[t]{2}{*}{2} & Titanium & 22 & 100.14 & 98.64 & 3.45 \\
\hline & & & Carbon & 6 & 1.38 & 1.36 & 0.23 \\
\hline \multirow[t]{4}{*}{7} & \multirow{4}{*}{$\begin{array}{l}\text { Control } \\
\text { sample }\end{array}$} & \multirow[t]{2}{*}{1} & Titanium & 22 & 101.05 & 98.80 & 3.49 \\
\hline & & & Carbon & 6 & 1.23 & 1.20 & 0.21 \\
\hline & & \multirow[t]{2}{*}{2} & Titanium & 22 & 101.34 & 98.78 & 3.50 \\
\hline & & & Carbon & 6 & 1.25 & 1.22 & 0.21 \\
\hline \multirow[t]{4}{*}{7} & \multirow{4}{*}{$\begin{array}{l}\text { Experiment } \\
\text { sample }\end{array}$} & \multirow[t]{2}{*}{1} & Titanium & 22 & 104.06 & 99.16 & 3.59 \\
\hline & & & Carbon & 6 & 0.88 & 0.84 & 0.16 \\
\hline & & \multirow[t]{2}{*}{2} & Titanium & 22 & 109.45 & 99.29 & 3.77 \\
\hline & & & Carbon & 6 & 0.78 & 0.71 & 0.15 \\
\hline \multirow[t]{4}{*}{8} & \multirow{4}{*}{ Control } & \multirow[t]{2}{*}{1} & Titanium & 22 & 105.24 & 99.53 & 3.62 \\
\hline & & & Carbon & 6 & 0.47 & 0.47 & 0.10 \\
\hline & & \multirow[t]{2}{*}{2} & Titanium & 22 & 106.24 & 99.26 & 3.66 \\
\hline & & & Carbon & 6 & 0.79 & 0.74 & 0.13 \\
\hline \multirow[t]{5}{*}{8} & \multirow{5}{*}{$\begin{array}{l}\text { Experiment } \\
\text { sample }\end{array}$} & 1 & Titanium & 22 & 105.30 & 99.56 & 3.63 \\
\hline & & & Carbon & 6 & 0.46 & 0.44 & 0.09 \\
\hline & & 2 & Titanium & 22 & 103.18 & 98.22 & 3.55 \\
\hline & & & Carbon & 6 & 1.30 & 1.24 & 0.18 \\
\hline & & & copper & 29 & 0.57 & 0.54 & 0.11 \\
\hline 9 & Control & 1 & Titanium & 22 & 105.24 & 99.53 & 3.62 \\
\hline & & & Carbon & 6 & 0.47 & 0.47 & 0.10 \\
\hline & & 2 & Titanium & 22 & 106.24 & 99.26 & 3.66 \\
\hline & & & Carbon & 6 & 0.79 & 0.74 & 0.13 \\
\hline 9 & Experiment & 1 & Titanium & 22 & 102.28 & 99.15 & 3.52 \\
\hline & sample & & Carbon & 6 & 0.88 & 0.85 & 0.14 \\
\hline & & 2 & Titanium & 22 & 103.34 & 99.08 & 3.56 \\
\hline & & & Carbon & 6 & 0.96 & 0.92 & 0.15 \\
\hline Titanium & & 1 & Titanium & 22 & 104.21 & 87.15 & 3.59 \\
\hline loaded with & & & Oxygen & 8 & 13.58 & 11.36 & 1.67 \\
\hline Air $(20 \%$ & & & Carbon & 6 & 1.78 & 1.49 & 0.27 \\
\hline oxygen) & & 2 & Titanium & 22 & 88.57 & 80.81 & 3.06 \\
\hline & & & Oxygen & 8 & 19.33 & 17.63 & 2.30 \\
\hline & & & Carbon & 6 & 1.70 & 0.26 & 0.26 \\
\hline & & 1 & Titanium & 22 & 96.38 & 82.63 & 3.33 \\
\hline
\end{tabular}




\begin{tabular}{|c|c|c|c|c|c|c|}
\hline \multirow{5}{*}{$\begin{array}{l}\text { Titanium } \\
\text { loaded with } \\
\text { Helium }\end{array}$} & & Oxygen & 8 & 15.99 & 13.71 & 1.94 \\
\hline & & Carbon & 6 & 4.28 & 3.67 & 0.56 \\
\hline & \multirow[t]{3}{*}{2} & Titanium & 22 & 113.64 & 89.98 & 3.92 \\
\hline & & Oxygen & 8 & 11.73 & 9.29 & 1.47 \\
\hline & & Carbon & 6 & 0.93 & 0.73 & 0.17 \\
\hline \multirow{4}{*}{$\begin{array}{l}\text { Titanium } \\
\text { loaded with } \\
\text { deuterium } \\
\text { in the } \\
\text { presence of } \\
\text { Air }\end{array}$} & \multirow[t]{2}{*}{1} & Titanium & 22 & 112.01 & 99.17 & 3.86 \\
\hline & & Carbon & 6 & 0.93 & 3.22 & 0.17 \\
\hline & \multirow[t]{2}{*}{2} & Titanium & 22 & 113.33 & 99.02 & 3.90 \\
\hline & & Carbon & 6 & 1.12 & 0.98 & 0.19 \\
\hline
\end{tabular}




\section{Appendix B}

Appendix B contains the calibration data of the helium-3 detectors used during this work. Helium-3 detectors have a high efficiency for detecting thermal neutrons. Since the neutron used for our calibration was a fast neutron, the helium-3 detector was enclosed in a polyethylene sleeve to moderate the neutron. The reaction between a thermal neutron and helium-3 leads to the production of a triton, ${ }_{1}^{3} \mathrm{H}$ and a proton, ${ }_{1}^{1} p$ released in opposite direction. The triton and proton share the $0.764 \mathrm{MeV}$ Q-value, with the proton taking 0.573 $\mathrm{MeV}$, and triton taking $0.191 \mathrm{MeV}$ of the total energy. The peaks of $0.764 \mathrm{MeV}$ can be seen in the pulse height distribution in the calibration data. Two helium-3 detectors and a proton recoil detector were used. The calibration curves are shown in the figures below.
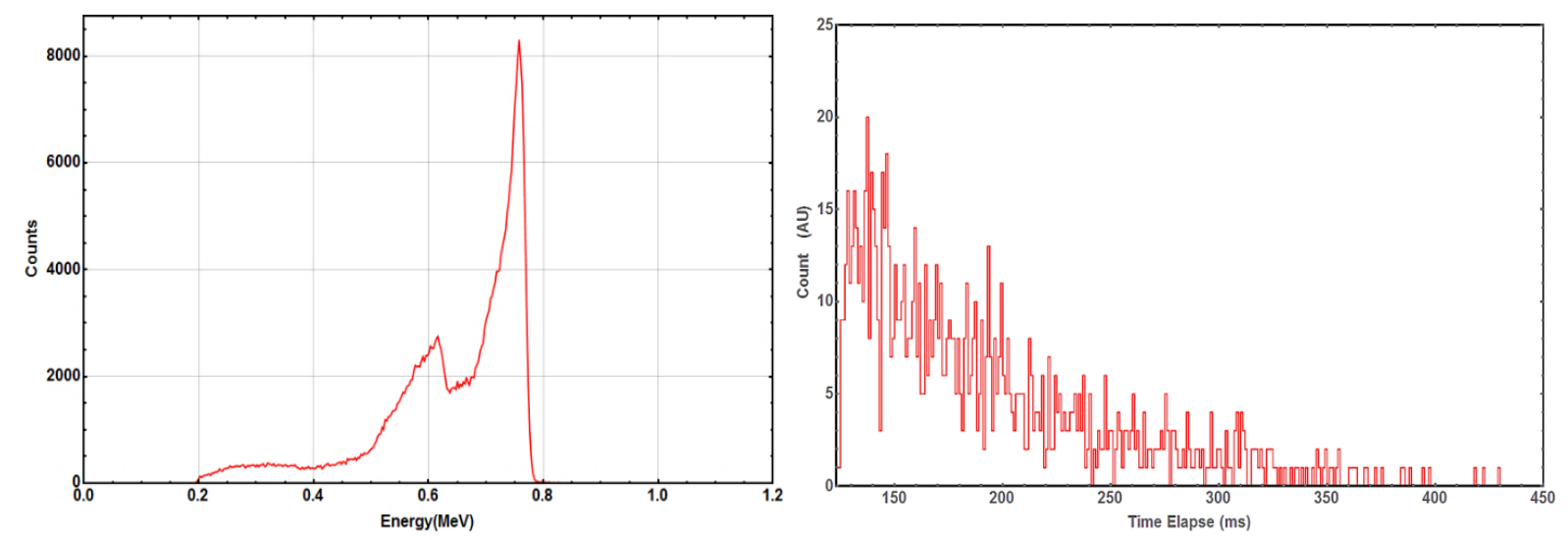

Figure A.I -Helium-3 Calibration Data [ Exp. 2 \& 3] and Unfolded Proton Recoil [ Experiment 1-2]

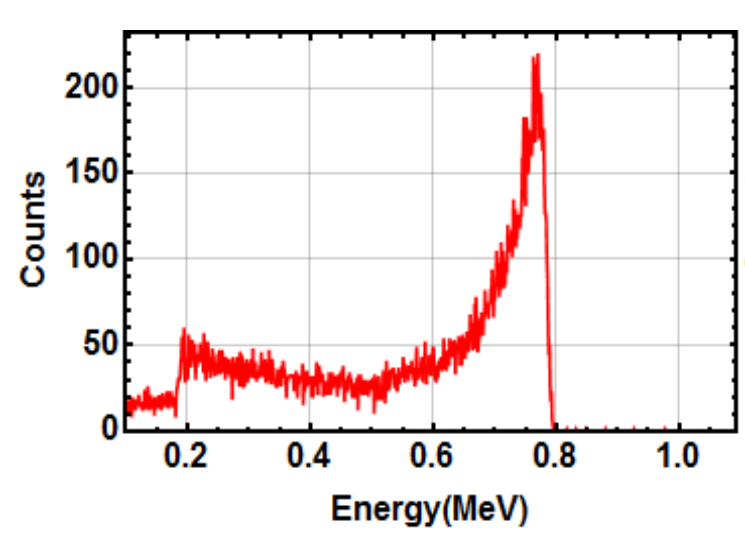

Detector 1

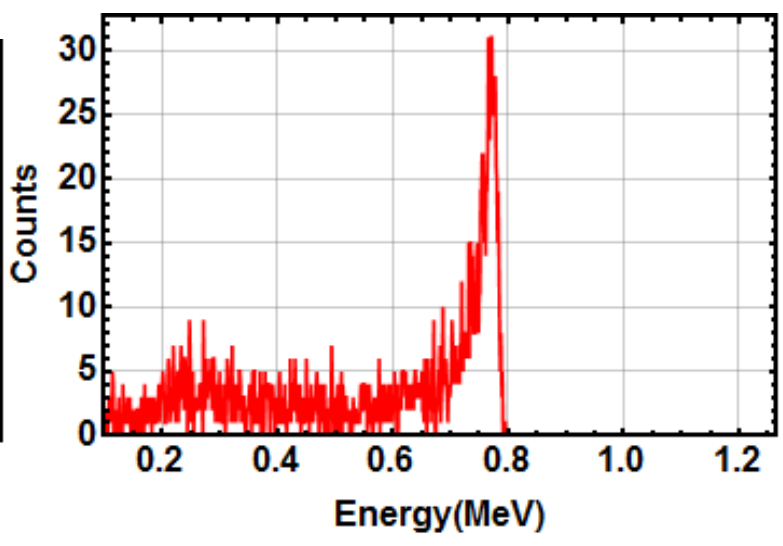

Detector 2 
Figure A.II -Calibration Data for Experiment 2- 5

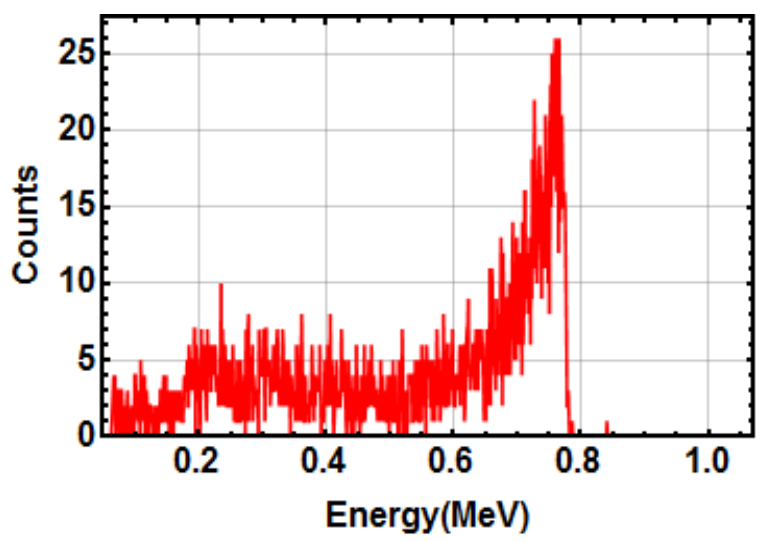

Detector 1

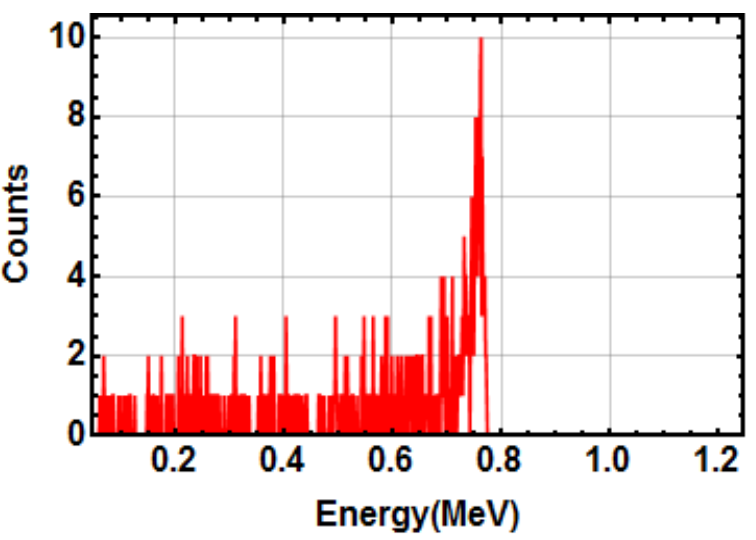

Detector 2

Figure A.III -Calibration Data for Experiment 7

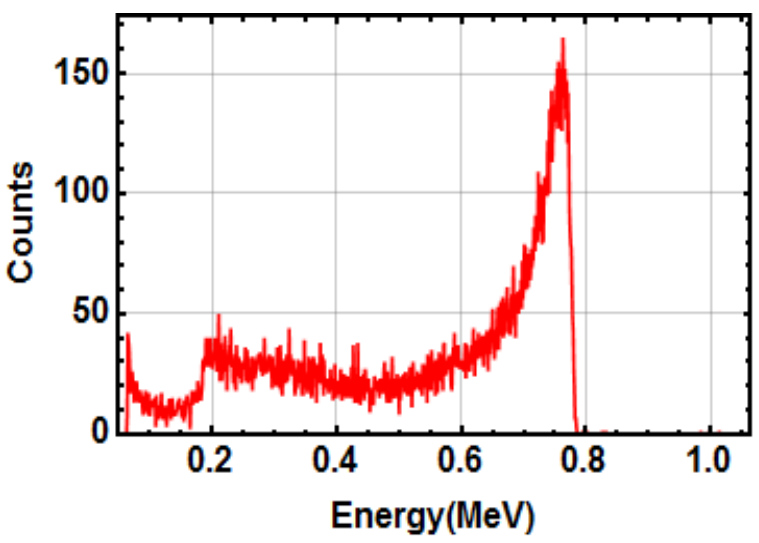

Detector 1

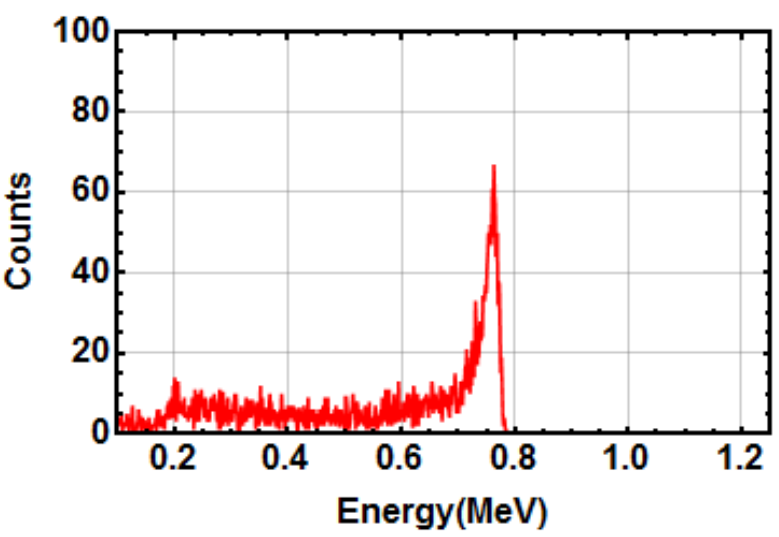

Detector 2

Figure A.IV -Calibration Data for Experiment 6
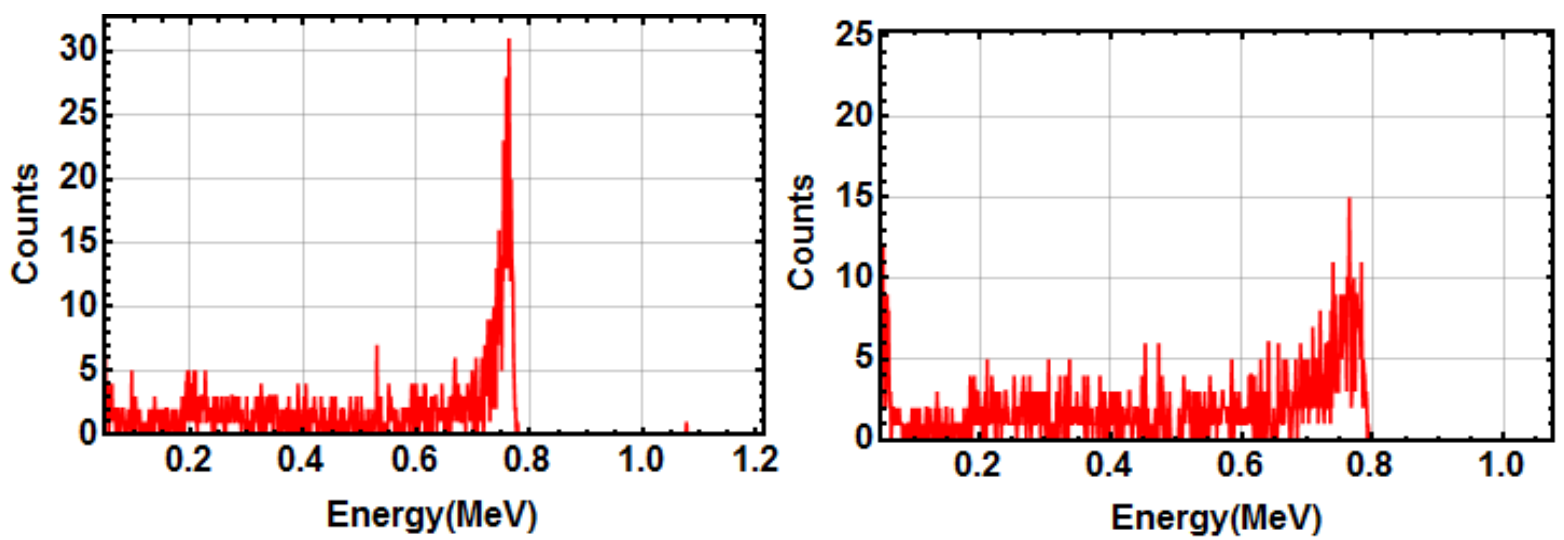
Detector 1

Detector 2

Figure A.V -Calibration Data for Experiment 7

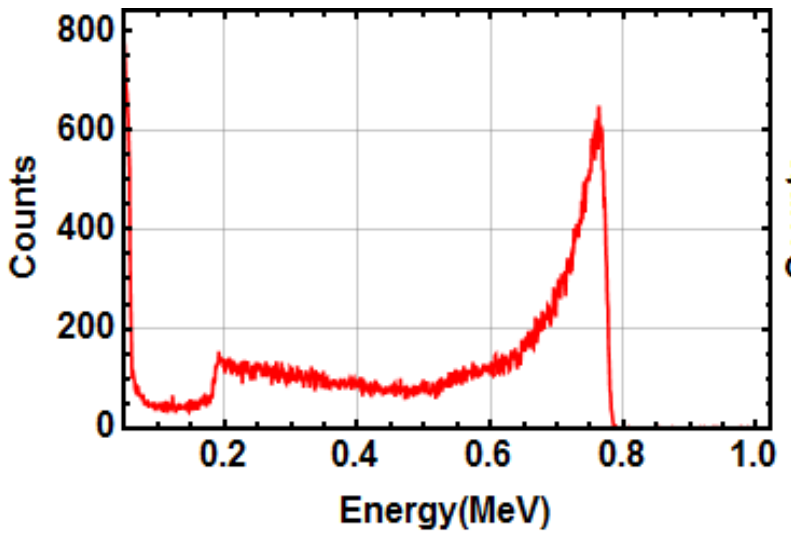

Detector 1

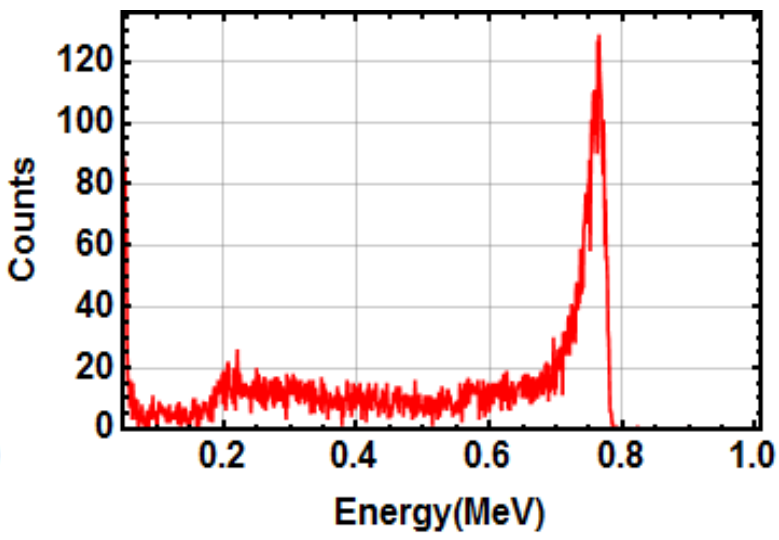

Detector 2

Figure A.VI -Calibration Data for Experiment 8

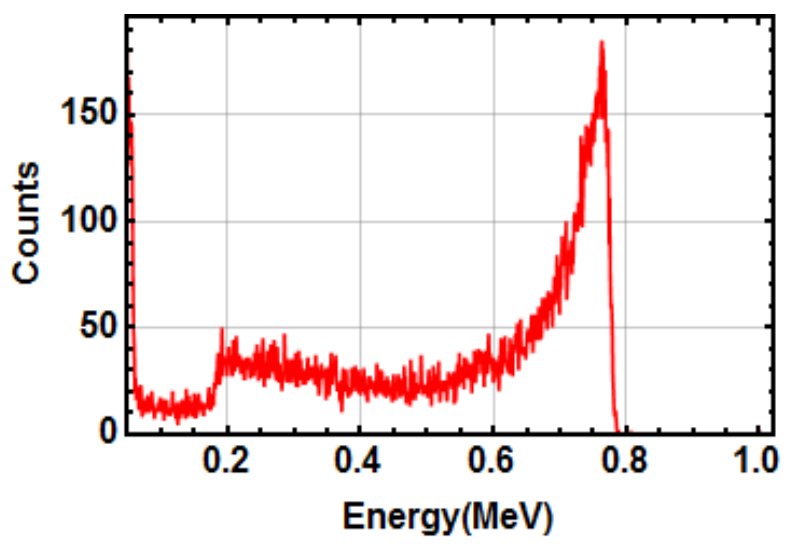

Detector 1

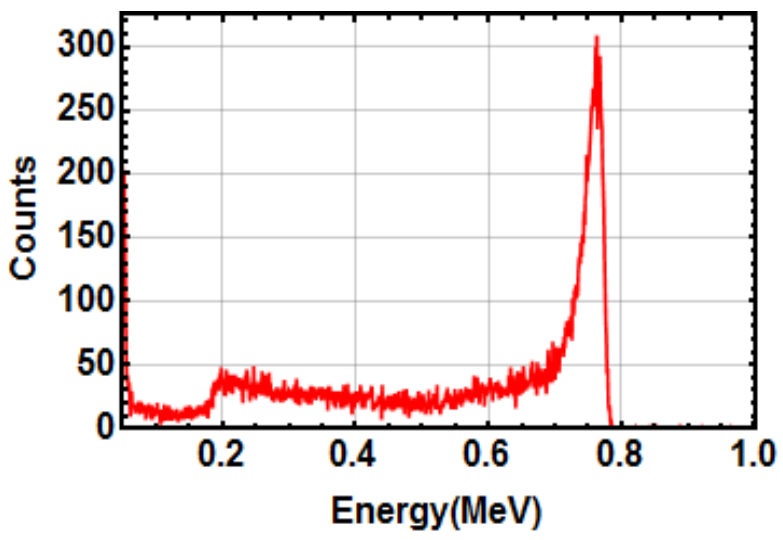

Detector 2

Figure A.VII -Calibration Data for Experiment 9 

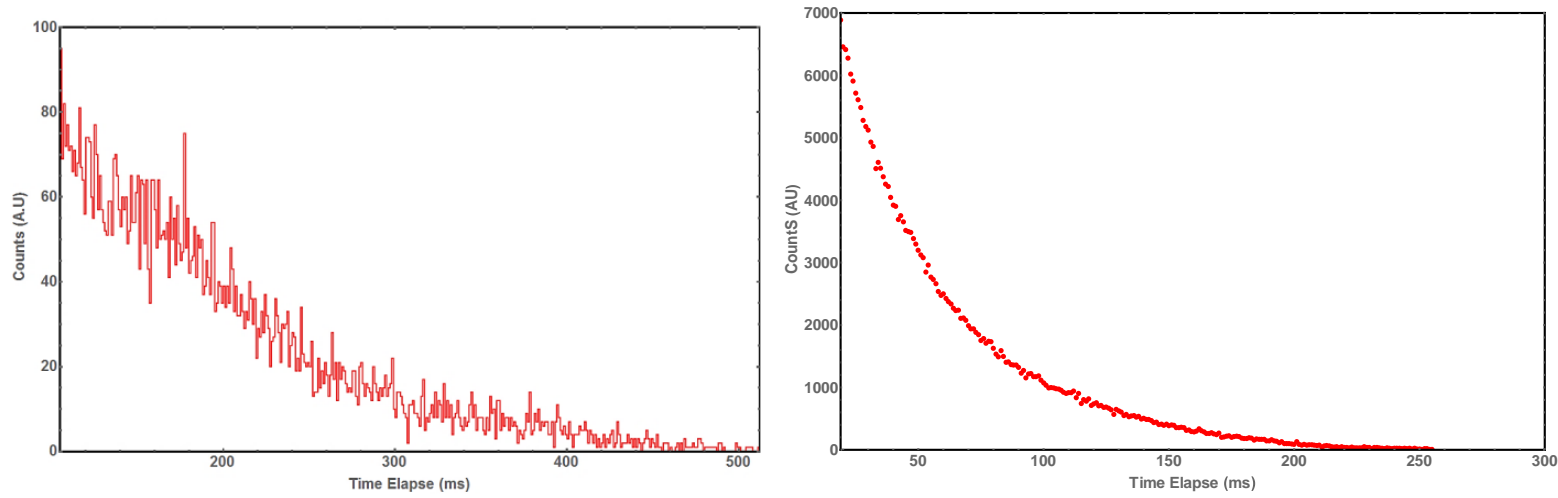

Figure A.I -Unfolded Proton Recoil Spectra, Left [ Exp. 5 \& 7], Right [ Experiment 8 \&9 


\section{Appendix C}

Appendix C shows the SEM images of experimental and control Samples. Several scanning electron microscope images of the titanium samples were taken throughout this work. Within this thesis, part of those images is in the results section. Because there were several regions showing crack formations within the samples, it important to show images of other part of the samples where likely titanium-deuterium reaction can occur. The images in Appendix B are for different regions in titanium samples other than those seen in the results section.
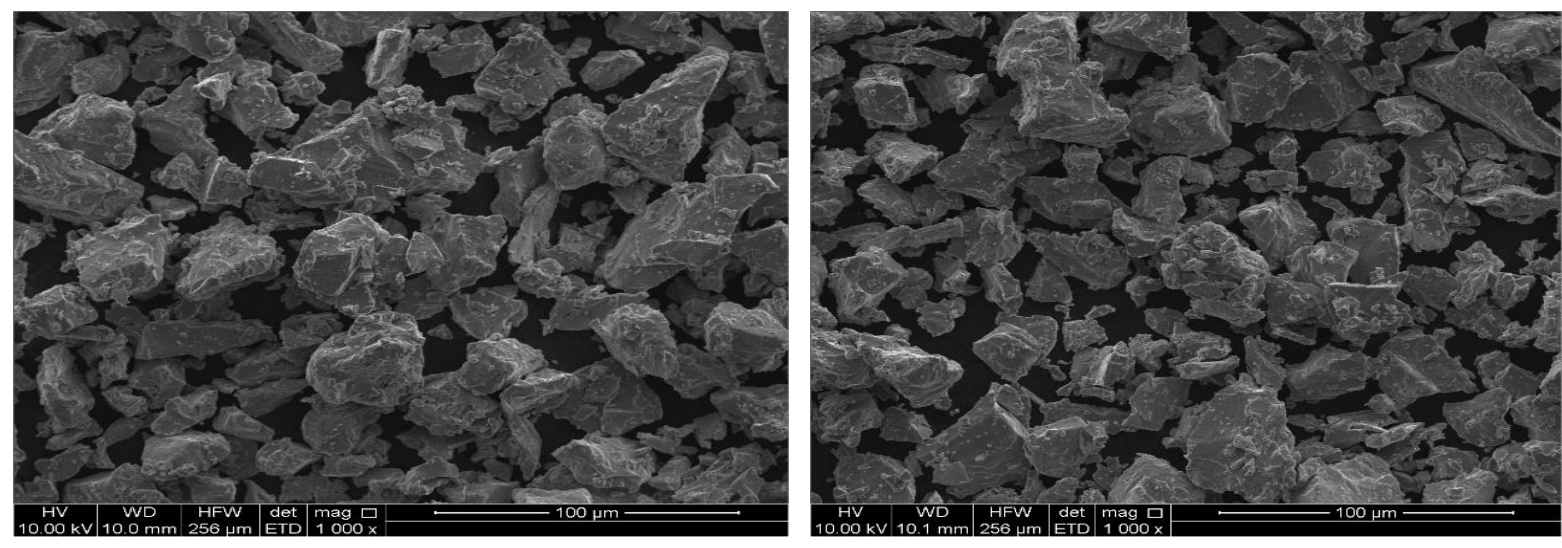

Figure B.I- SEM Images of Experiment 1, Control (left) and Experimental (right)
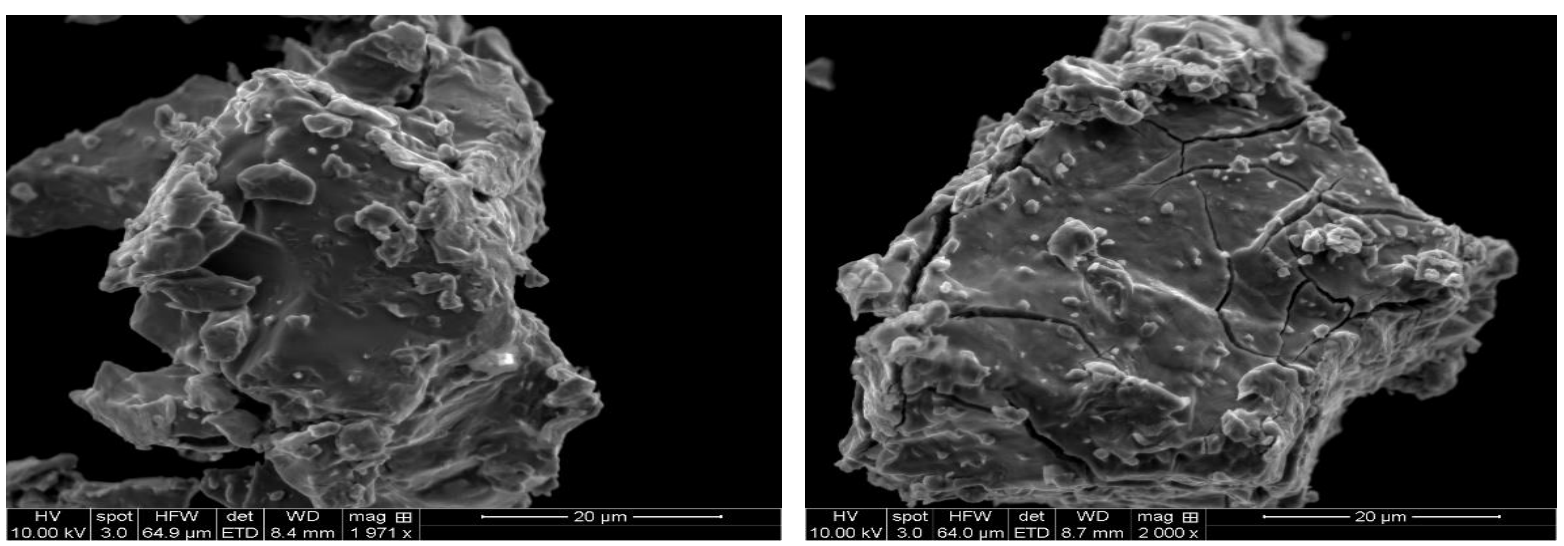

Figure B.II- SEM Images of Experiment 2, Control (left) and Experimental (right) 

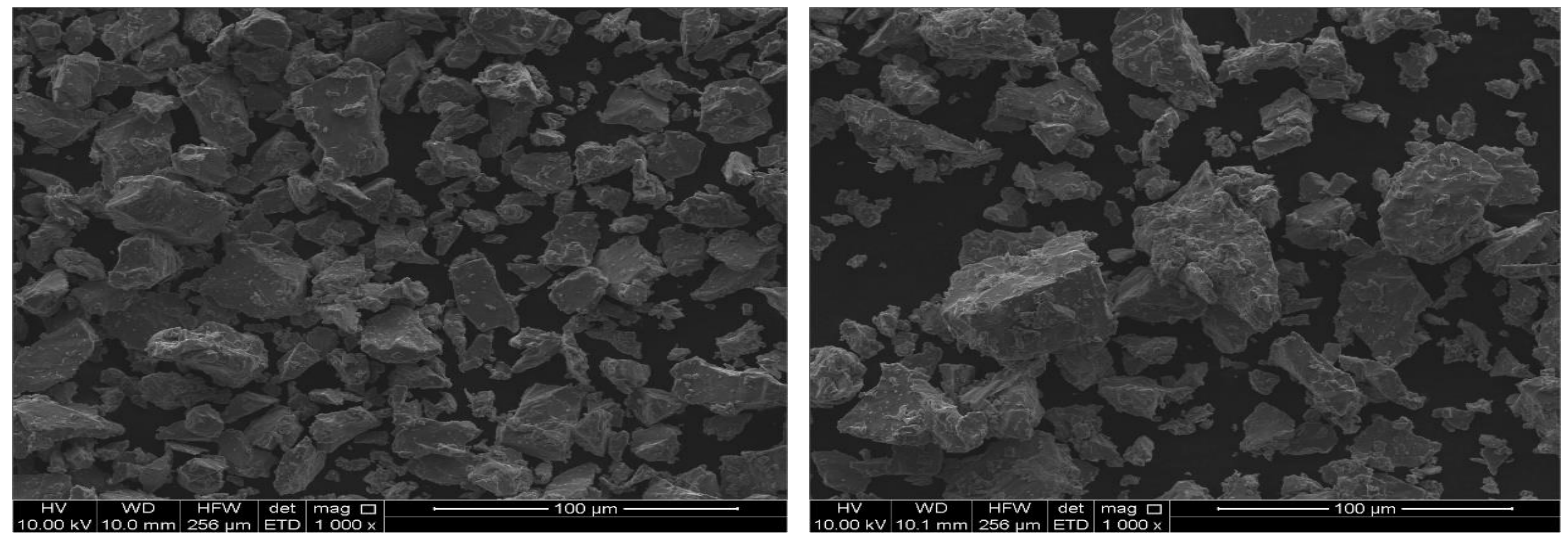

Figure B.III- SEM Images of Experiment 3, Control (left) and Experimental(right)
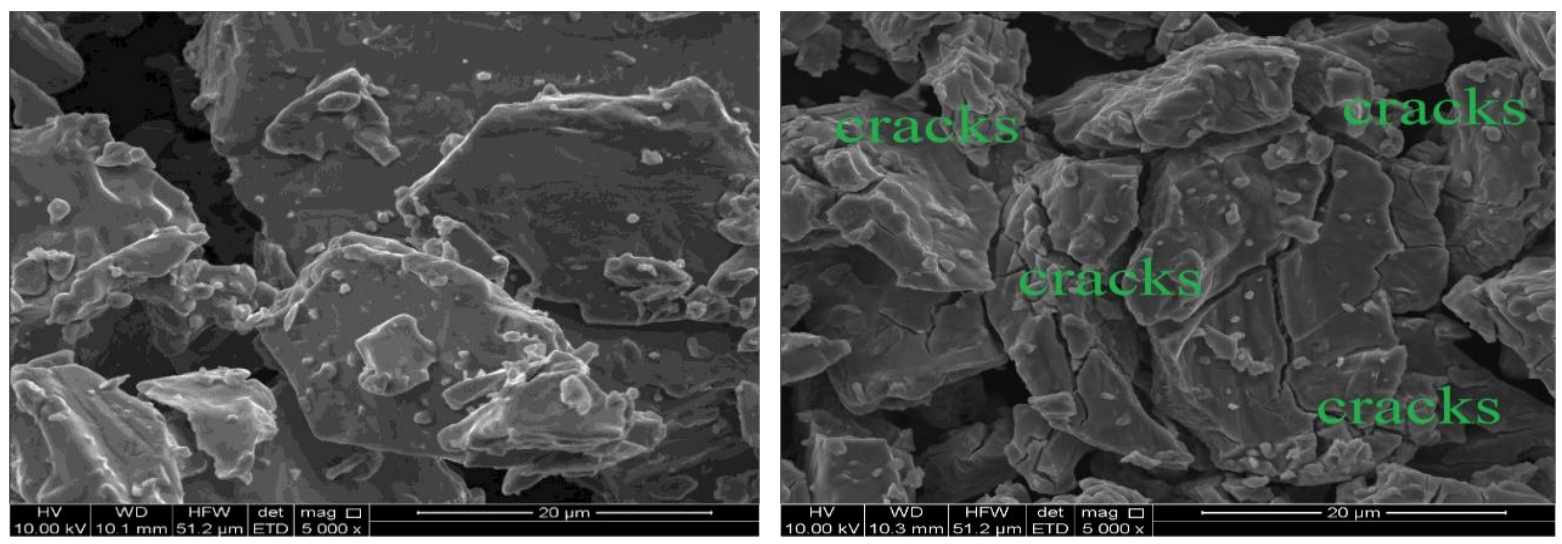

Figure B.IV- SEM Images of Experiment 4, Control (left) and Experimental (right)
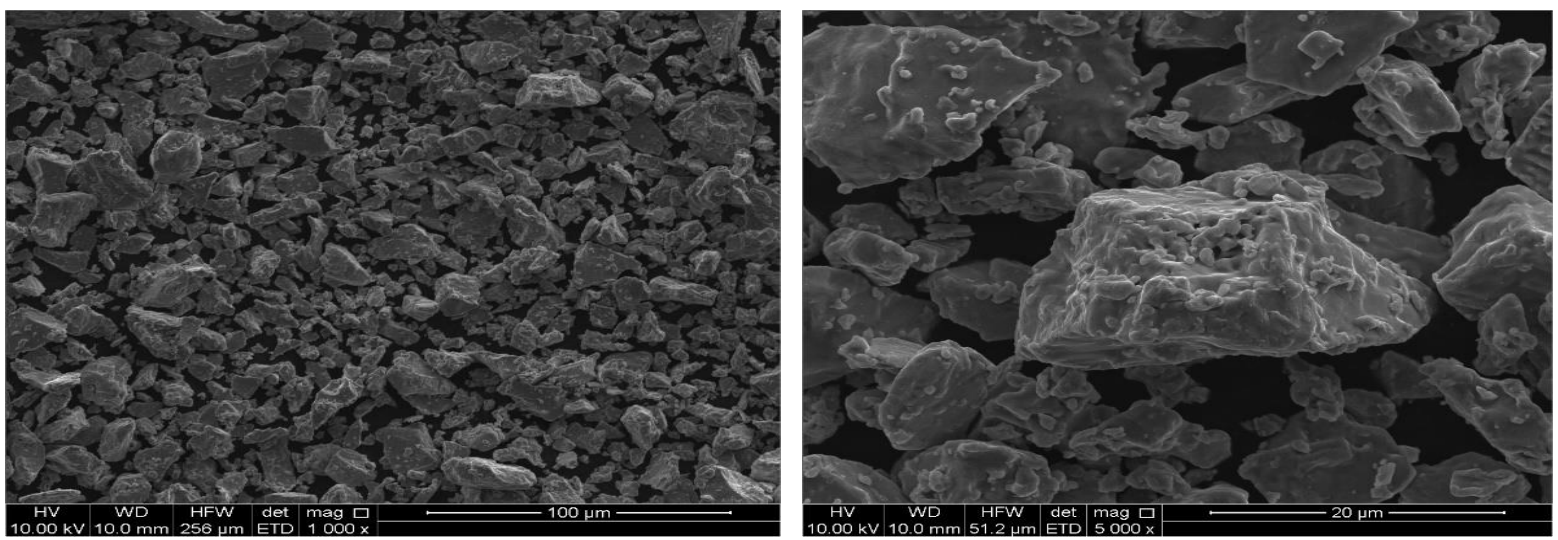

Figure B.IV- SEM Images of Experiment 5, Control (left) and Experimental (right) 

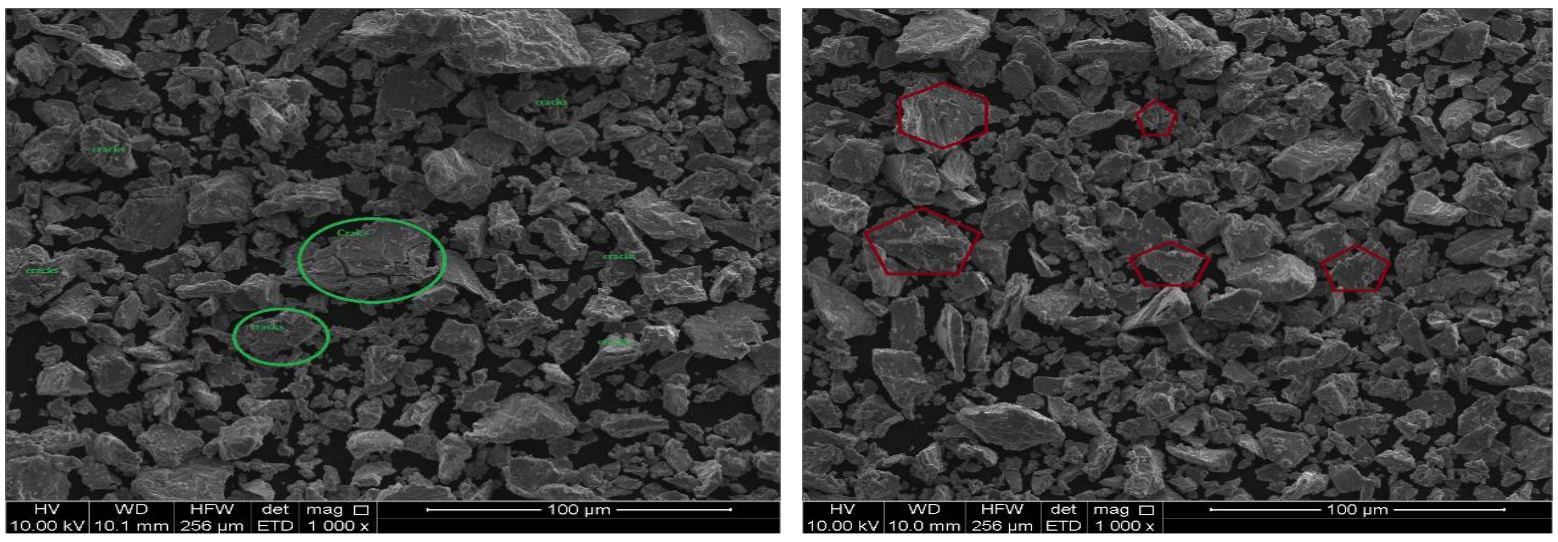

Figure B.V- SEM Images of Experiment 6, Control (left) and Experimental (right)
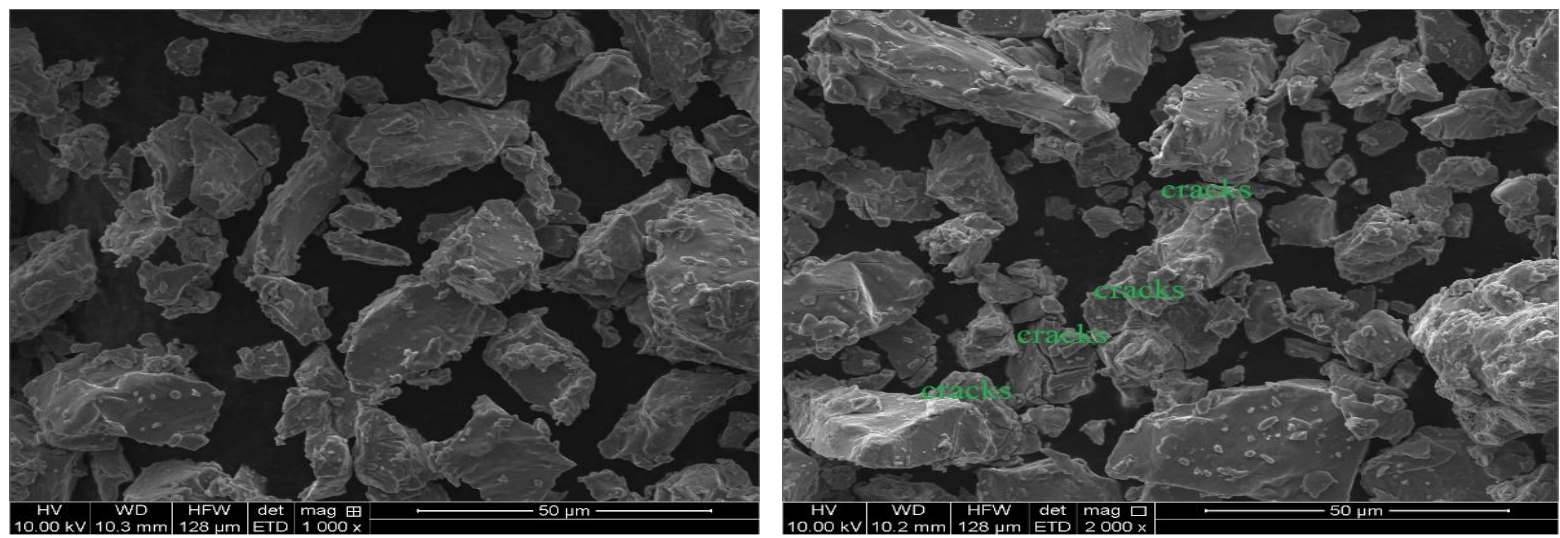

Figure B.VI- SEM Images of Experiment 7 , Control (left) and Experimental (right)
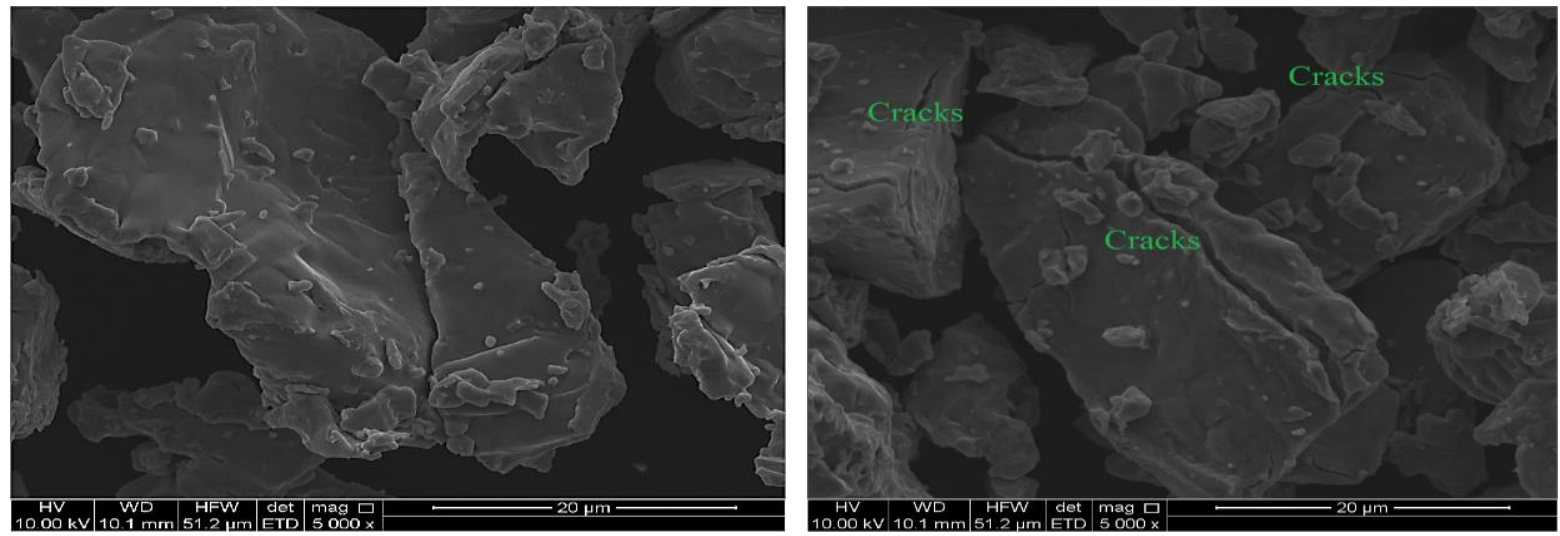

Figure B.VII- SEM Images of Experiment 8, Control (left) and Experimental (right) 

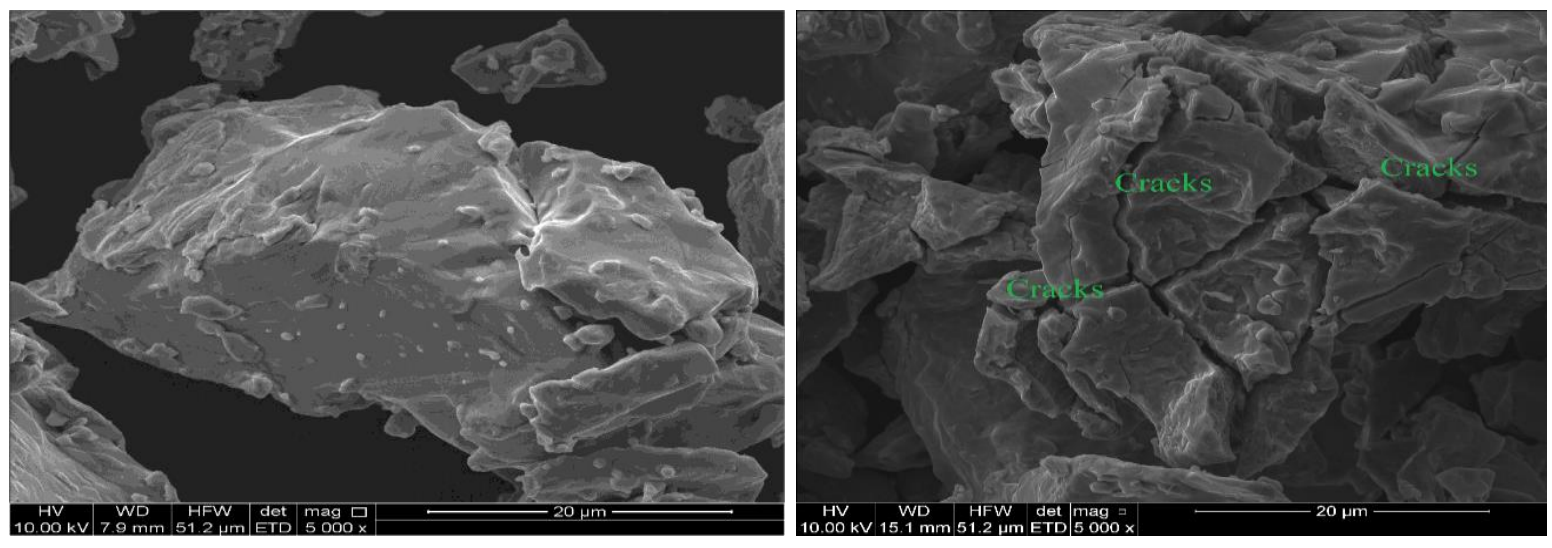

Figure B.IX- SEM Images of Experiment 9, Control (Left) and Experimental (Right)

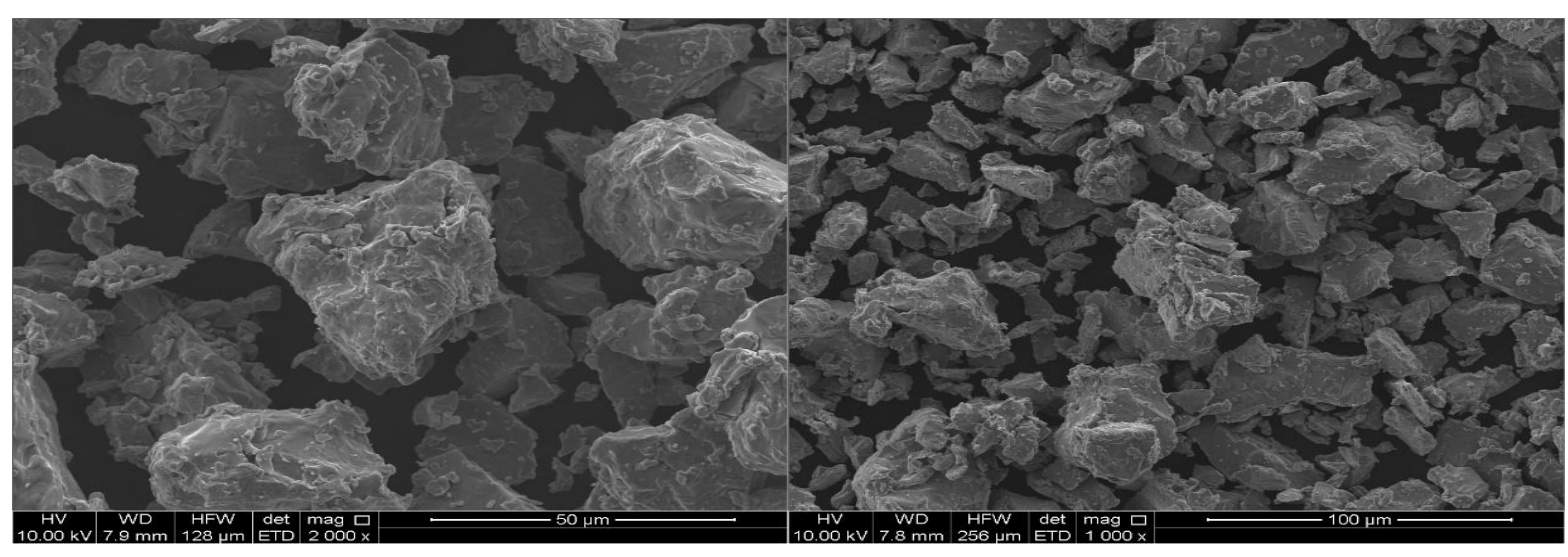

Figure B.X- SEM Images of Experiment 10, Control (Left) and Experimental (Right) 


\section{Appendix D}

Appendix D shows the EDS images of positions used in elemental analysis.

Determining the composition of elements in titanium through the energy dispersive

spectroscopy method requires surface mapping of different particles in the titanium sample.

The point shot method was used to analyze the sample -where a particle or region in the sample is chosen for such analysis. Because this analysis method does not involve a large portion of the sample, there are errors involved using this method. Three regions were randomly selected for such analysis as shown in the images shown below.
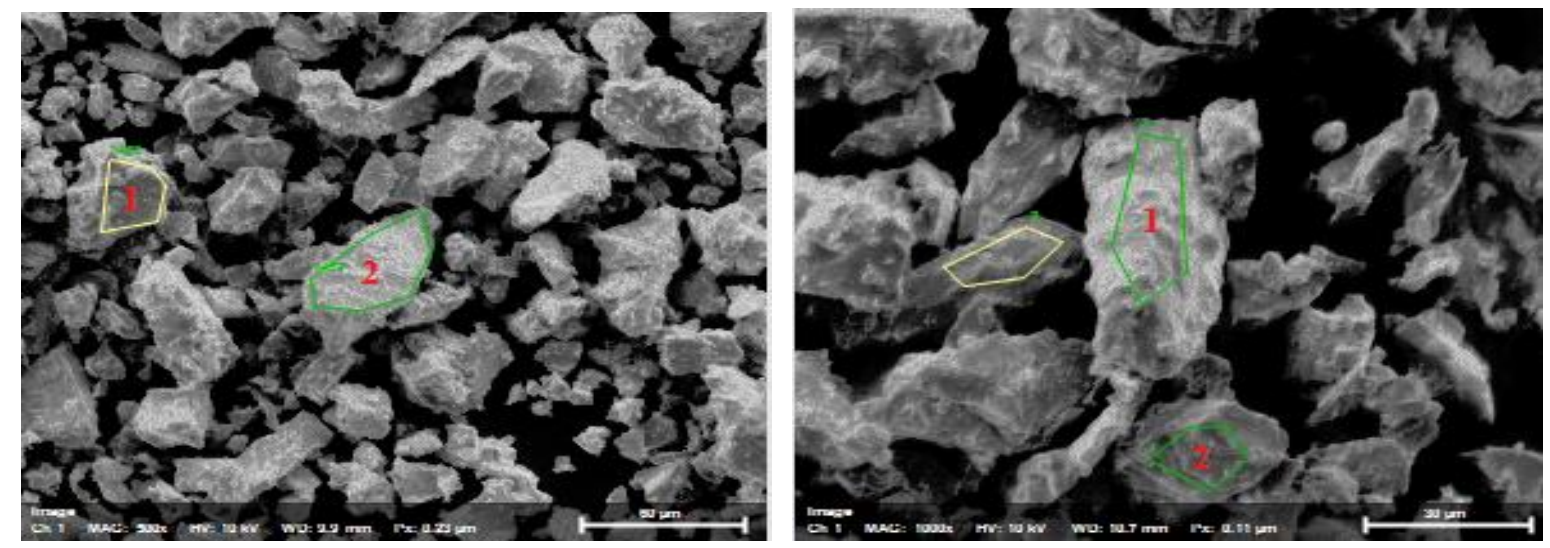

Figure D.I- EDS Images of showing analyzed position of Experiment 1, Control (Left) and Experimental (Right)
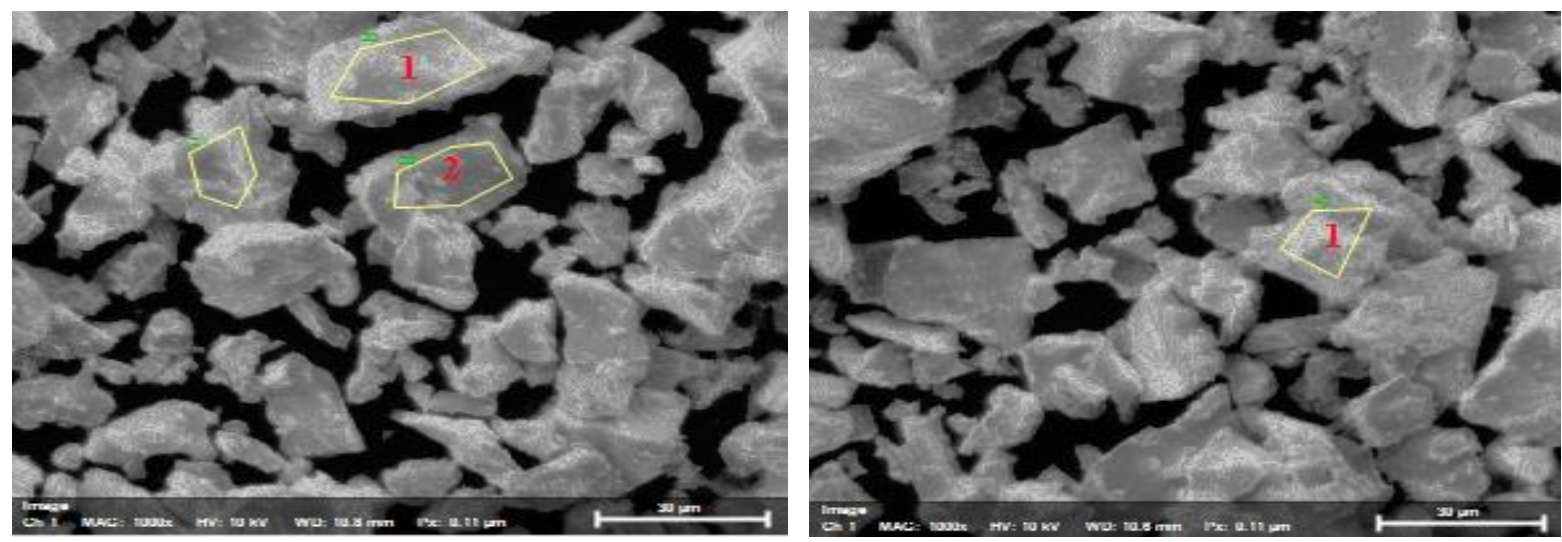

Figure D.II- EDS Images of showing analyzed position of Experiment 2, Control (Left) and Experimental (Right) 

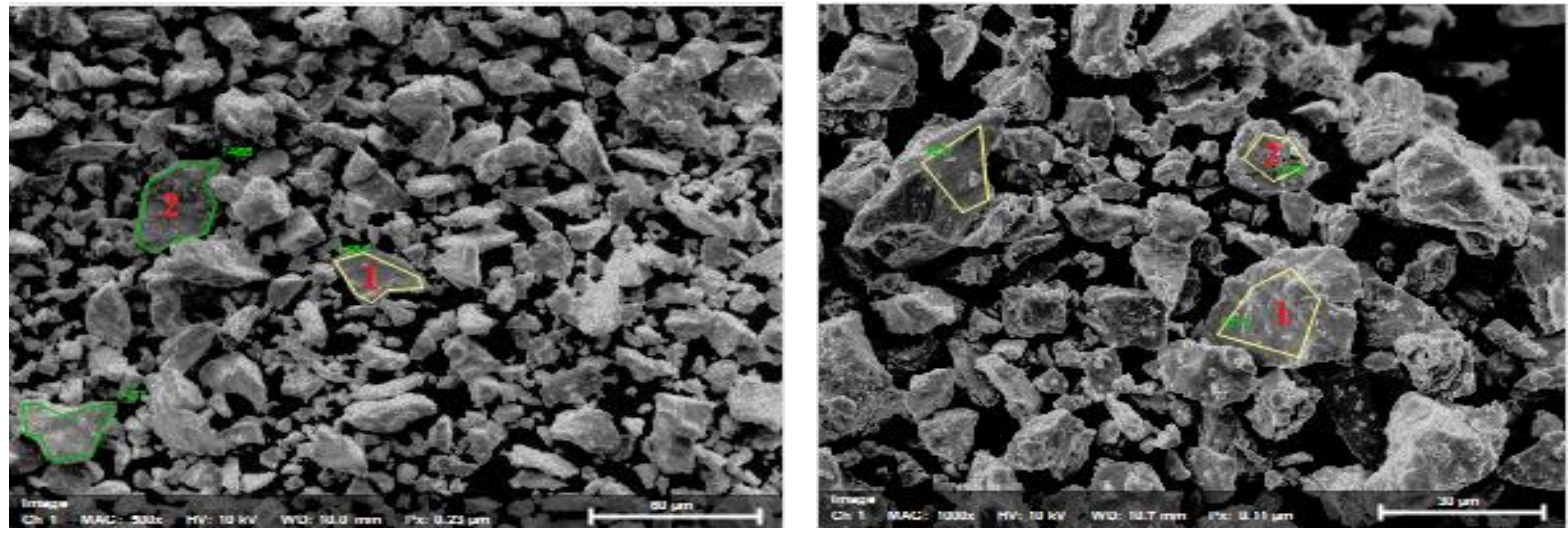

Figure D.V- EDS Images of showing analyzed position of Experiment 5, Control (Left) and Experimental (Right)
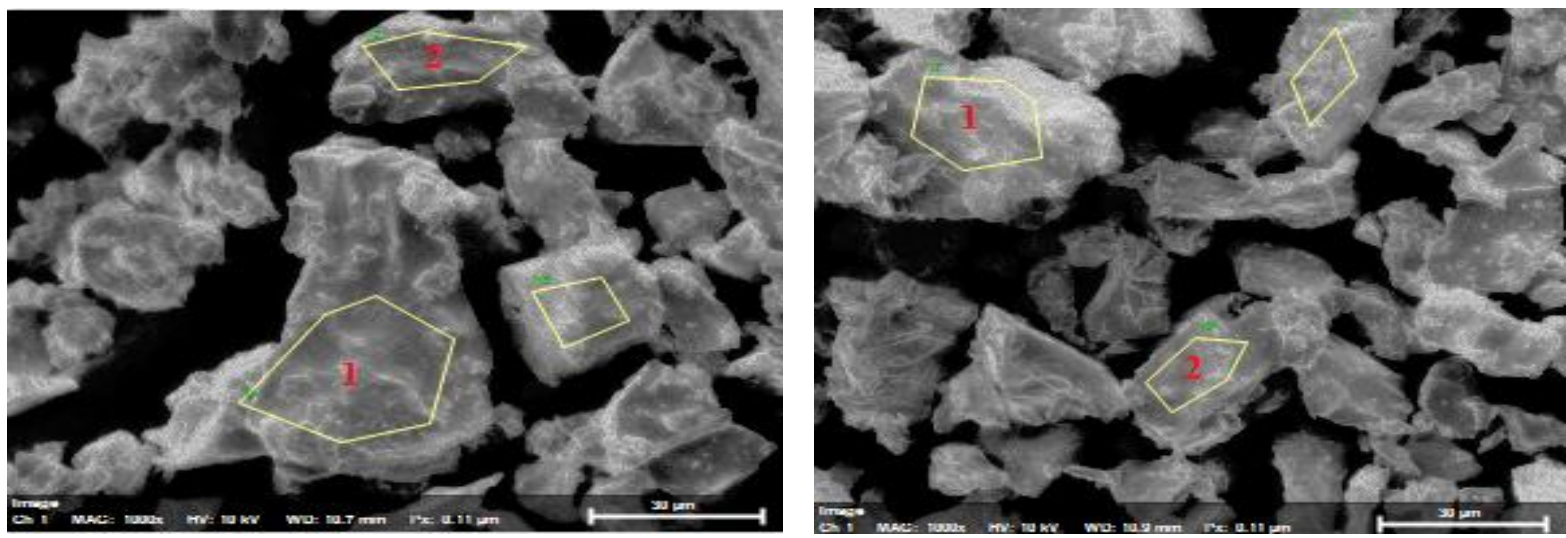

Figure D.VI- EDS Images of showing analyzed position of Experiment 6, Control (Left) and Experimental (Right)
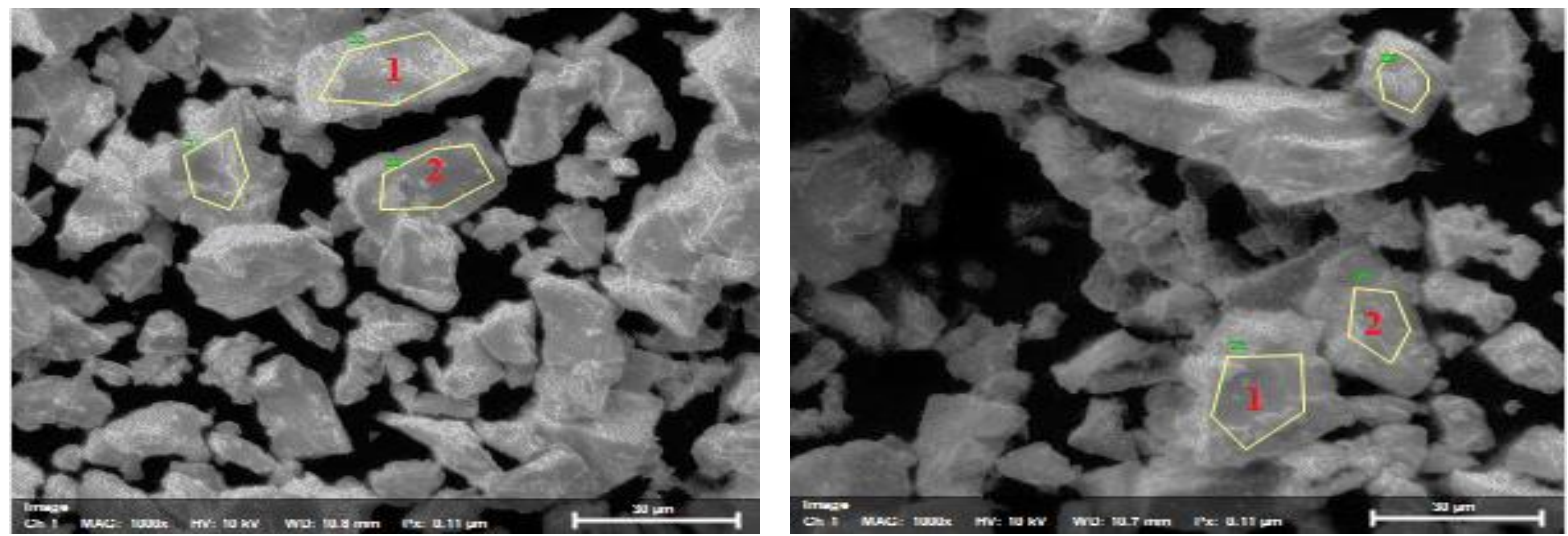

Figure D.VII- EDS Images of showing analyzed position of Experiment 7, Control (Left) and Experimental (Right) 

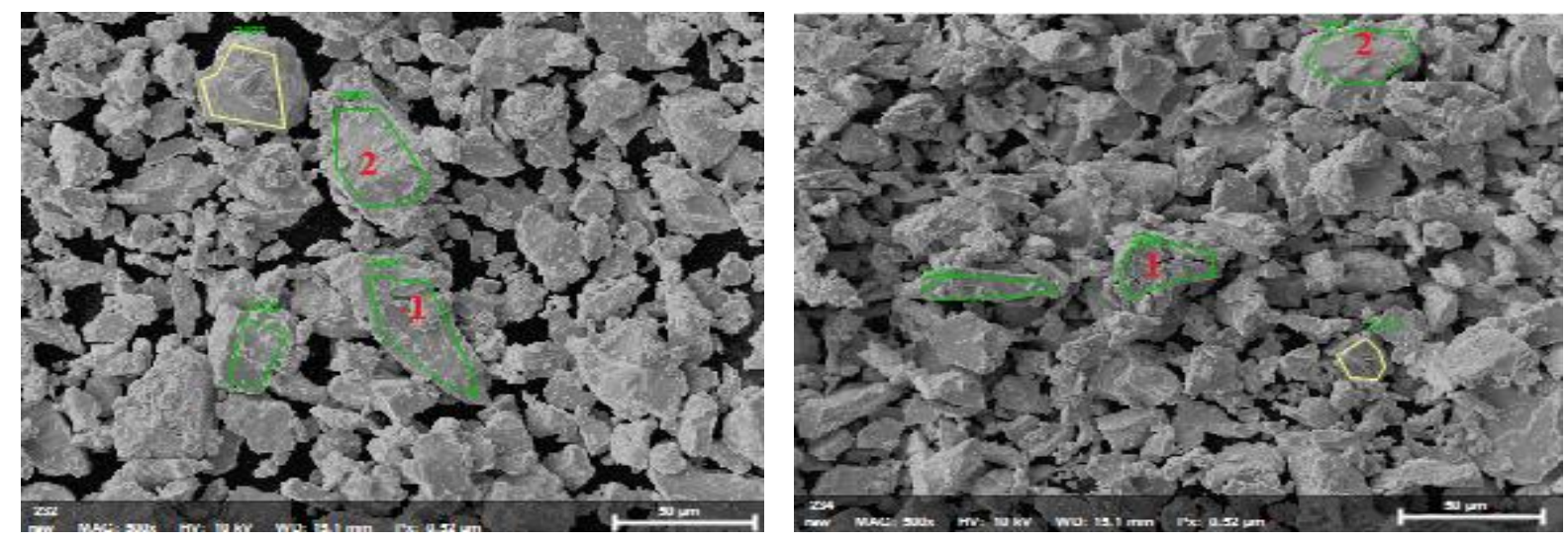

Figure D.VIII- EDS Images of showing analyzed position of Experiment 8, Control (Left) and Experimental (Right)
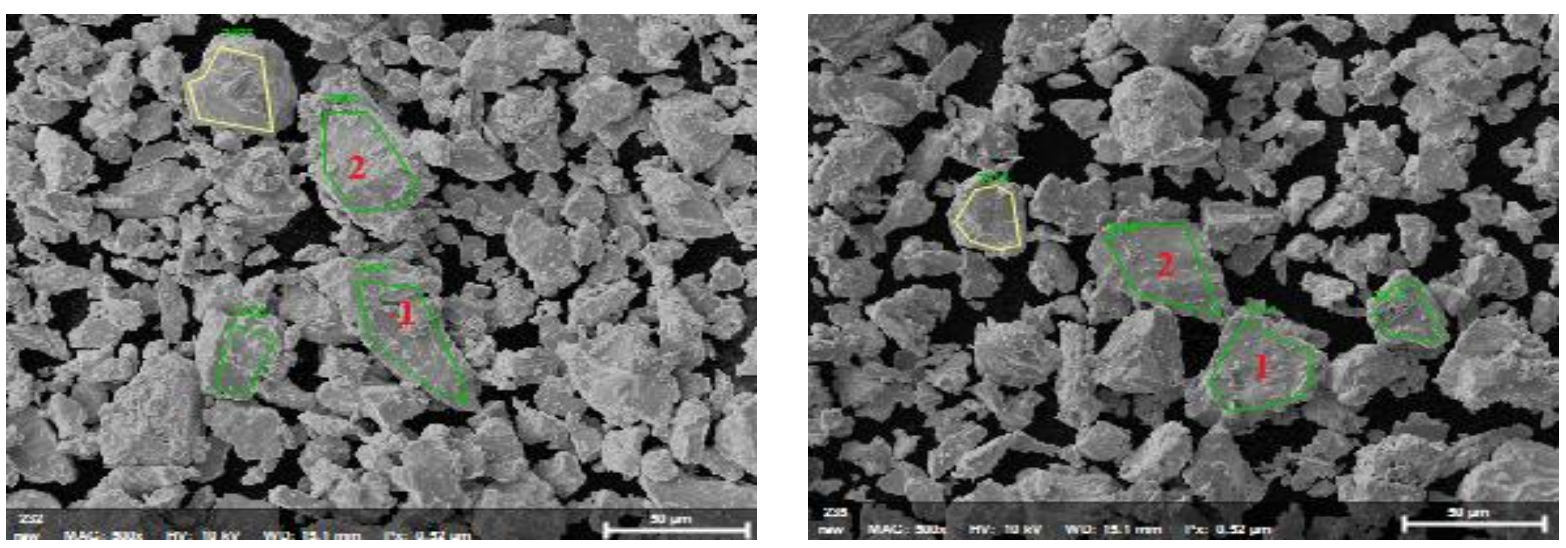

Figure D.IX- EDS Images of showing analyzed position of Experiment 9, Control (Left) and Experimental (Right) (EDS analysis region)
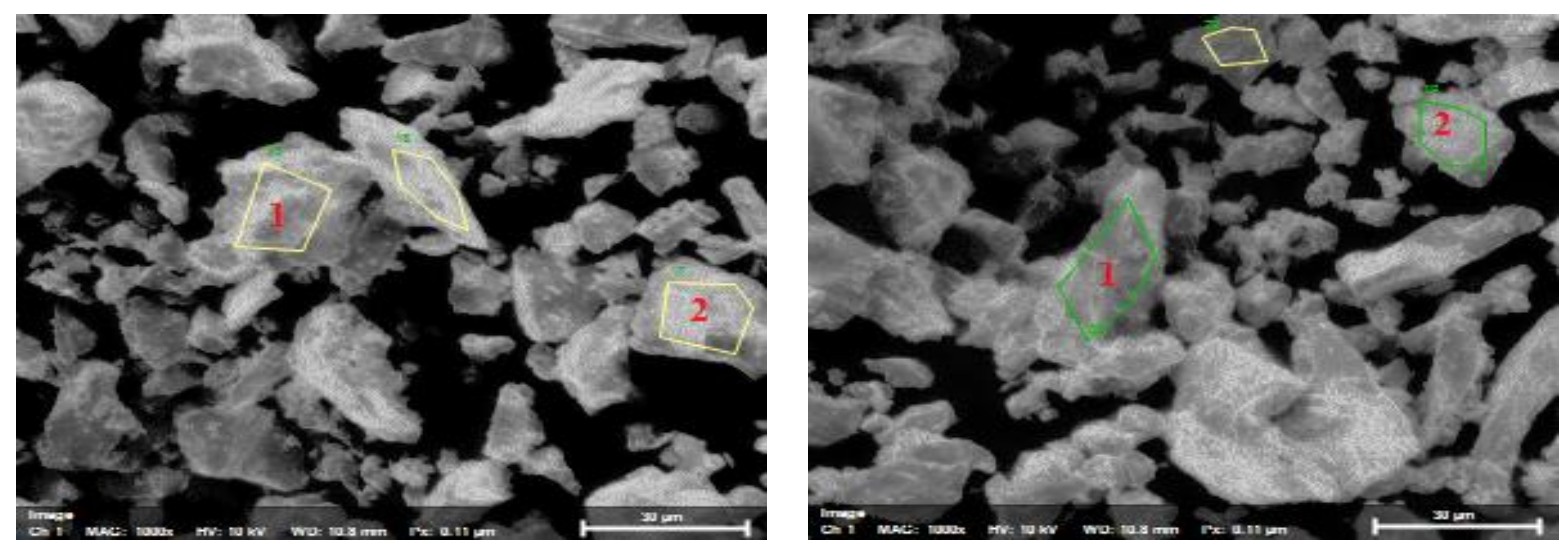

Figure D.X- EDS Images showing analyzed position of Experiment 1, Titanium loaded with air (Left) andTitanium loaded under helium (Right) 


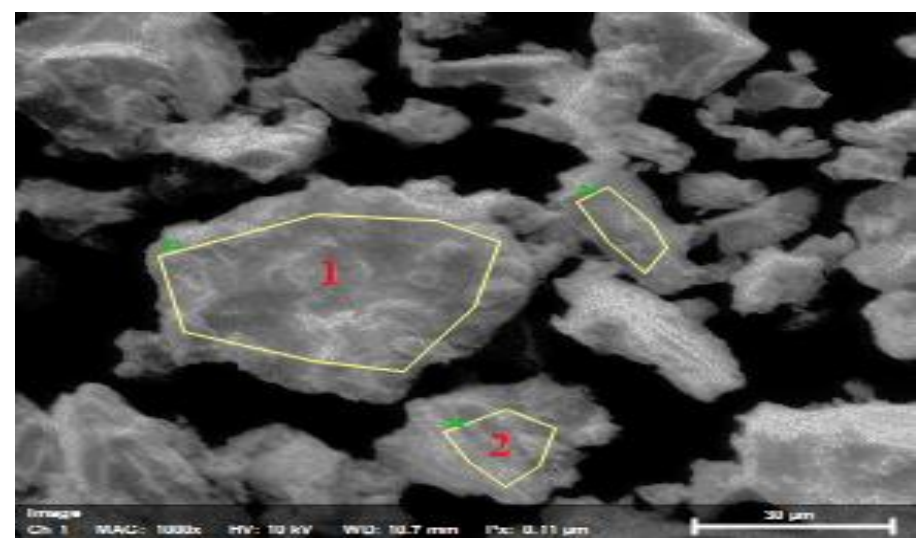

Figure D.I- EDS Images of showing analyzed position of Titanium loaded in the presence of oxygen

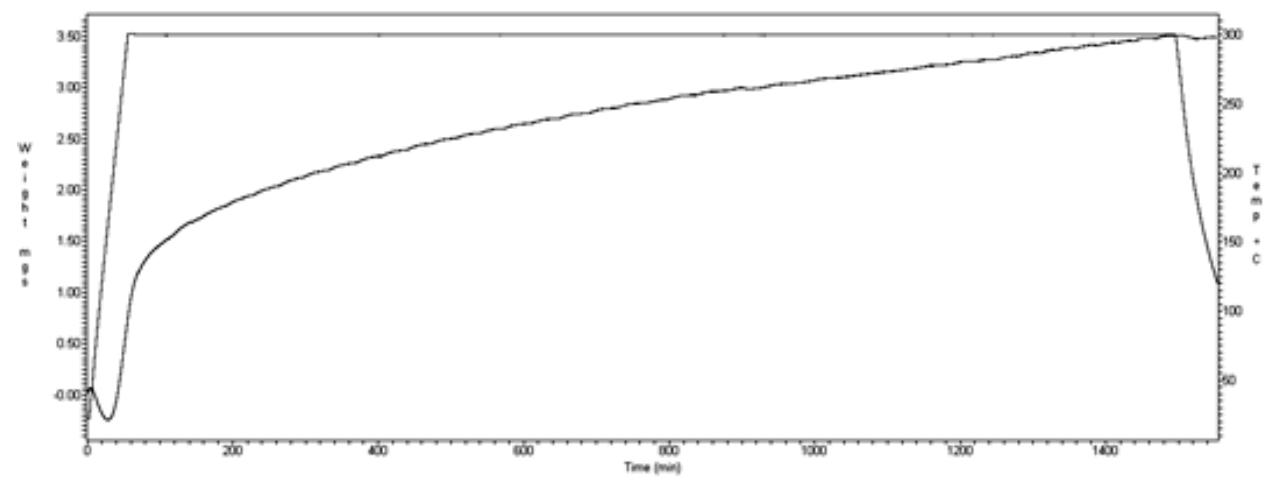

TGA analysis for Titanium powder under helium

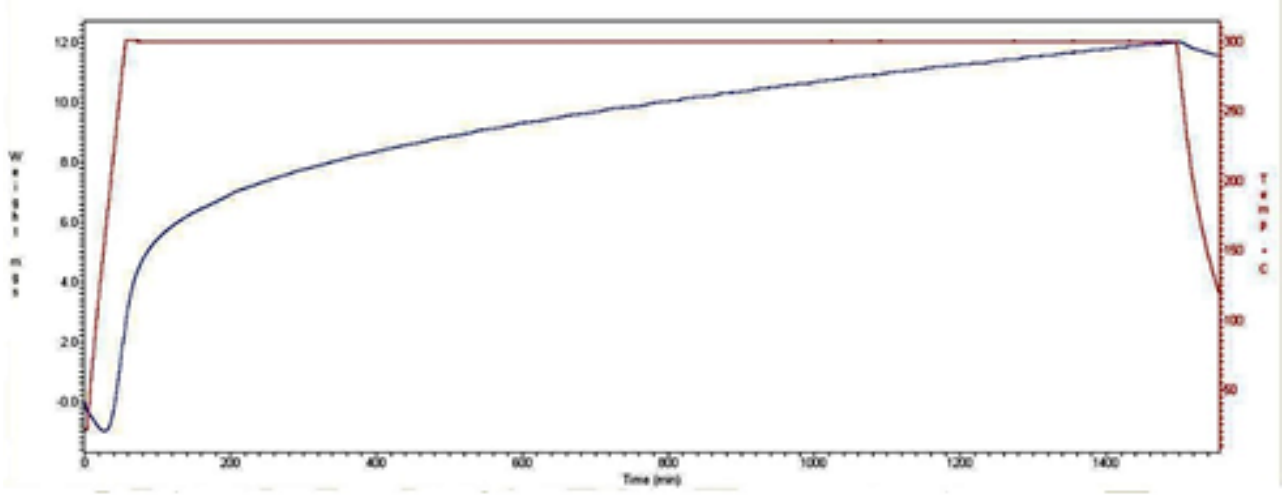

TGA analysis for Titanium powder under air 


\section{VITA}

Modeste Tchakoua was born in Tombel, Cameroon on the afternoon of April 2, 1988. He completed a bachelor's degree in Physics and a minor in computer science at the University of Buea, Cameroon in 2010, where graduated as the top student in his department. He came to the United States through the prestigious Fulbright scholarship program to pursue a master's degree in Nuclear Engineering at the University of Missouri and graduated in July 2014. He found great admiration for the work of Dr. Mark A. Prelas and decided to stay to work for his Ph. D. under his supervision. Modeste will finish his doctoral degree in December 2017. 